\title{
MR imaging of neo-vasculature
}

Citation for published version (APA):

de Lussanet-de la Sabloniere, Q. G. (2005). MR imaging of neo-vasculature. [Doctoral Thesis, Maastricht University]. Universitaire Pers Maastricht. https://doi.org/10.26481/dis.20051209qd

Document status and date:

Published: 01/01/2005

DOI:

10.26481/dis.20051209qd

Document Version:

Publisher's PDF, also known as Version of record

\section{Please check the document version of this publication:}

- A submitted manuscript is the version of the article upon submission and before peer-review. There can be important differences between the submitted version and the official published version of record.

People interested in the research are advised to contact the author for the final version of the publication, or visit the DOI to the publisher's website.

- The final author version and the galley proof are versions of the publication after peer review.

- The final published version features the final layout of the paper including the volume, issue and page numbers.

Link to publication

\footnotetext{
General rights rights.

- You may freely distribute the URL identifying the publication in the public portal. please follow below link for the End User Agreement:

www.umlib.nl/taverne-license

Take down policy

If you believe that this document breaches copyright please contact us at:

repository@maastrichtuniversity.nl

providing details and we will investigate your claim.
}

Copyright and moral rights for the publications made accessible in the public portal are retained by the authors and/or other copyright owners and it is a condition of accessing publications that users recognise and abide by the legal requirements associated with these

- Users may download and print one copy of any publication from the public portal for the purpose of private study or research.

- You may not further distribute the material or use it for any profit-making activity or commercial gain

If the publication is distributed under the terms of Article $25 \mathrm{fa}$ of the Dutch Copyright Act, indicated by the "Taverne" license above, 


\section{MR imaging of neo-vasculature}

Quido G. de Lussanet de la Sablonière 
(9) Quido Gisou de Lussanet de la Sablonière, Maastricht 2005 QdLu@rdia.azm.nl

ISBN $90-5278-502-3$

Printed by: Datawyse / Universitaire Pers Maastricht

Financial support for this thesis was generously provided by Philips Medical Systems, GE Healthcare, Guerbet Nederland BV, Schering Nederland BV, Kodak. 


\title{
MR imaging of neo-vasculature
}

\author{
Proefschrift
}

ter verkrijging van de graad van doctor

aan de Universiteit Maastricht, op gezag van de Rector Magnificus

prof. mr. G.P.M.F. Mols,

volgens het besluit van het College van Decanen,

in thet openbaar te verdedigen

op vrijdag 9 december 2005 om 14.00 uur

door

Quido Gisou de Lussanet de la Sablonière geboren op 27 september 1973 te Amersfoort

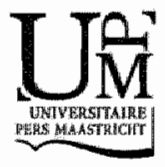


Promotor

Prof.dr. J.M.A. van Engelshoven

Copromotores

Dr. R.G.H. Beets-Tan

Dr. W.H. Backes

Beoordelingscommissie

Prof.dr. M.J. Past, voorzitter

Prof.dr. M.J.A.P. Daemen

Prof.dr. E.W. Meijer (Technische Universiteit Eindhoven)

Prof.dr. J.F.M. Smits

The research was funded in part by the Cardiovascular Research Institute Maastricht (CARIM), Maastricht, The Netherlands. 


\section{Contents}

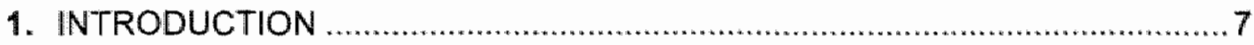

INTRODUCTION

REFERENCES 9

OBJECTIVE OF THIS THESIS 10

OUTLINE OF THIS THESIS

2. GADOPENTETATE DIMEGLUMINE VERSUS USPIO FOR DYNAMIC CONTRAST-ENHANCED MRI OF TUMOR ANGIOGENESIS IN HUMAN COLON CARCINOMA IN MICE

$\begin{array}{lr}\text { ABSTRACT } & 11 \\ \text { INTRODUCTION } & 12 \\ \text { MATERIALS AND METHODS } & 12 \\ \text { RESULTS } & 16 \\ \text { DISCUSSION } & 20 \\ \text { APPENDIX } & 24 \\ \text { REFERENCES } & 26\end{array}$

3. RELATIONSHIP BETWEEN DCE-MRI KINETIC PARAMETERS AND MOLECULAR WEIGHT OF DENDRITIC CONTRAST AGENTS IN TUMOR ANGIOGENESIS IN MICE

$\begin{array}{ll}\text { ABSTRACT } & 29 \\ \text { INTRODUCTION } & 30 \\ \text { MATERIALS AND METHODS } & 31 \\ \text { RESULTS } & 33 \\ \text { DISCUSSION } & 38 \\ \text { REFERENCES } & 42\end{array}$

4. DYNAMIC CONTRAST-ENHANCED MRI AT 1.5 TESLA WITH

GADOPENTETATE DIMEGLUMINE TO ASSESS ANGIOSTATIC EFFECTS OF ANGINEX IN MICE

$\begin{array}{ll}\text { ABSTRACT } & 43 \\ \text { INTRODUCTION } & 44 \\ \text { MATERIALS AND METHODS } & 45 \\ \text { RESULTS } & 47 \\ \text { DISCUSSION } & 49 \\ \text { REFERENCES } & 52\end{array}$

5. DYNAMIC CONTRAST-ENHANCED MAGNETIC RESONANCE IMAGING OF RADIATION THERAPY INDUCED MICROCIRCULATION CHANGES IN RECTAL CANCER.

ABSTRACT

INTRODUCTION

PATIENTS AND METHODS $\quad 54$

RESULTS

DISCUSSION

$\begin{array}{ll}\text { REFERENCES } & 62\end{array}$ 
6. DYNAMIC CONTRAST-ENHANCED MR IMAGING OF PERFUSION

RECOVERY AND MR ANGIOGRAPHY OF COLLATERAL GROWTH IN A FEMORAL ARTERY LIGATION MODEL

ABSTRACT 65

INTRODUCTION

MATERIALS AMD METHODS 66

RESULTS $\quad 69$

$\begin{array}{ll}\text { DISCUSSION } & 72\end{array}$

REFERENCES $\quad 75$

7. MAGNETIC RESONANCE ANGIOGRAPHY OF COLLATERAL VESSEL GROWTH IN A RABBIT FEMORAL ARTERY LIGATION MODEL …............77

$\begin{array}{ll}\text { ABSTRACT } & 77\end{array}$

$\begin{array}{ll}\text { INTRODUCTION } & 78\end{array}$

MATERIALS AND METHODS $\quad 78$

RÉSULTS

DISCUSSION

REFERENCES 88

8. EVALUATION OF GD(III)DTPA-TERMINATED POLY(PROPYLENE IMINE) DENDRIMERS AS CONTRAST AGENTS FOR MR IMAGING .....................89

ABSTRACT $\quad 89$

INTRODUCTION 90

MATERIALS AND METHODS $\quad 91$

RESULTS

DISCUSSION 98

REFERENCES 101

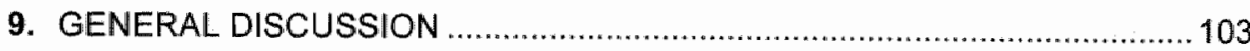

GENERAL DISCUSSION

REFERENCES $\quad 110$

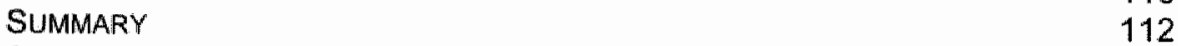

SAMENVATTING $\quad 115$

ACKNOWLEDGEMENTS $\quad 119$

CURRICULUM VITAE

LIST OF PUBLICATIONS 


\title{
Chapter 1
}

\author{
Introduction
}

\section{Introduction}

Magnetic resonance (MR) imaging may be regarded the imaging modality of choice for three-dimensional visualization of soft-tissue and disease morphology, in Western medicine . A new frontier in clinical MR imaging is to evaluate disease in terms of functional parameters to complement disease morphology ${ }^{2.3}$. In comparison with other established imaging techniques, such as CT, PET and ultrasound, MR imaging offers a superior combination of $3 \mathrm{D}$ imaging with high spatial resolution, low observer dependency, and without radiation or iodinated contrast agents that can incidentally cause nephrotoxicity ${ }^{4}$.

The specific MR imaging technique that pertains to this thesis is commonly referred to as dynamic contrast-enhanced MR imaging (DCE-MRI). The basis of DCE-MRI is to describe the dynamic changes in MR signal intensity in selected tissue volumes after intravenous injection of a MR contrast agent. These signal intensity changes are caused by the leakage of a circulating contrast agent (in the blood) into the tissue. By measuring the consequent signal intensity changes in a tissue volume " one can obtain a robust measure for tissue perfusion. For example, reductions in relative signal enhancement were shown to reflect inhibition of tumor angiogenesis by anti-angiogenesis therapy ${ }^{5}$. Such MR signal enhancement measures are limited however, because the measured MR signal intensity is influenced, for example, by the imaging parameters (e.g." echo time, repetition time, flip angle) and contrast agent dose and -injection rate. To perform more refined and diagnostic site independent DCE-MRll evaluations of tissue neovascularization, one should account for these dependencies.

The dependency of the measured MR signal internsity on the MR imaging parameters may be accounted for by converting signal intensity changes to contrast agent concentration changes, based on the relaxivity change due to contrast agent uptake. DCE-MRI derived parameters based on the contrast agent uptake include the initial area under the signal intensity time curve" (IAUC) (unit: $\mathrm{mM}$ Gd.s), which has been proposed as a recommended endpoint for use in Phase 1/2a trials for anticancer therapies, provided that contrast agent dose and-injection rate are reproducible ${ }^{6}$.

To account for the dependency of DCE-MRI derived measurements on contrast agent dose and-injection rate, the contrast agent concentration measurements in tissue and in blood may be evaluated using a general pharmacokinetic model 6,7. General pharmacokinetic models will render various parameters that reflect different microcirculation characteristics * A commonly used DCE-MRI derived parameter describes the rate of leakage of contrast agent from the blood into the tissue interstitial space, also known as the endothelial transfer coefficient $\left(k^{P S}\right.$ or $\left.k^{\text {trans }}\right)$. As with the IAUC, this parameter is a recommended endpoint for use in Phase $1 / 2 \mathrm{a}$ trials for anticancer therapies ${ }^{6}$.

The DCE-MRI derived pharmacokinetic parameters are more complex than they might appear, and it is currently unclear what these parameters measure exactly. The $K^{\text {pis }}$, for example is dependent on the microcirculatory flow, permeability and surface area. A 
question that yet remains to be answered is which of these properties $K^{\mathrm{PS}}$ reflects primarily. Moreover, it is speculated that the answer will depend on the particle size of the MR contrast agent used. Hence, this thesis explores the complex relationship between the molecular weight of the MR contrast agent used. The use of small particulate clinically available MR contrast agents is compared with the use of larger particulate experimental contrast agents for DCE-MRI to ultimately determine whether cinically available MR contrast agents may be used for DCE-MRI in different diseases or disease models.

\section{DCE-MRI in oncology}

DCE-MRI is increasingly used in oncology, to evaluate neo-vascularization ${ }^{10}$. Tumor development is highly dependent on neo-vascularization, a process that is also referred to as tumor angiogenesis "11. Tumor angiogenesis is characterized by initial increases in vessel wall permeability caused by vessel wall degradation, to allow migration of proliferated endothelial celis towards the angiogenic stimulus (tumor cells). Collections of migrated endothelial cells form new microvessels that ultimately remodel to form a mature vascular network. Neo-vascularization related changes in microvessel permeability, -surface area, -blood flow, and -blood volume are reflected by the DCEMRI derived parameters. Primary applications for DCE-MRI in oncology are to evaluate and predict tumor response to treatment targeted at the tumor vasculature.

DCE-MRI in arterial occlusive disease

If DCE-MRI is useful for evaluating neo-vascularization in tumors, then DCE-MRI might also be useful for evaluating neo-vascularization in other (disease-) conditions such as arthritis ${ }^{12}$, wound healling ${ }^{13}$, and perhaps arterial occlusive disease. Arterial occlusive disease is a common disease that can lead to myocardial infarction, stroke, or peripheral arterial disease. Neo-vascularization plays a crucial role in prevention or recovery of arterial occlusive disease. Just as treatments are being developed to inhibit neovascularization in oncology ${ }^{14}$, so are treatments being developed to stimulate neovascularization to promote recovery in arterial occlusive disease 15,16 . Neovascularization in arterial occlusive disease can be promoted in the microcirculation to form new capillaries (i.e., angiogenesis) and in arteriolar connections to form collateral vessels (i.e., arteriogenesis) 11. It is hypothesized that DCE-MRI can monitor microcirculation changes in a peripheral arterial occlusive disease model. Microcirculatory changes such as blood flow recovery after arterial occlusion may be related ether to capillary formation and $/$ or vessel growth. A combined protocol of DCEMRI and micromangiography that may visualize collateral vessels will be evaluated to determine whether MR imaging is useful for monitoring arterial occlusive disease in terms of functional parameters. 


\section{References}

1. 1.Gore J. Out of the shadows-MRI and the Nobel Prize. N Engl J Med. 2003;349:2290-2.

2. McDonald DM, Choyke PL. Imaging of angiogenesis: from microscope to clinic. Natl Mod: $2003 ; 9: 713-25$.

3. Pearlman JD, Laham RJ, Post M, Leiner T. Simons M. Medical imaging techniques the evaluation of strategies for therapeutic angiogenesis. Cum Pharm Des. 2002;8:1467-96.

4. Niendorf HP, Haustein J, Comelius I, Alhassan A, Clauss W. Safety of gadolintumtDTPA: extended clinical experience. Magn Reson Med. 1991;22:222-8; discussion 229-32.

5. van der Schaft DW, Dings RP, de Lussanet $Q$, van Eljk LI, Nap AW, Beets-Tan RG, Bouma-Ter Steege $\mathrm{JC}_{2}$ Wagstaff $J$ "Mayo $\mathrm{KH}$, Griffioen $\mathrm{AW}$. The designer anti-angiogenic peptide anginex targets tumor endothelial cells and inhibits tumor growth in animal models. Faseb $d$. $2002 ; 16: 1991-3$.

6. Leach MO, Brindle KM, Evelhoch JL, Griffiths JR, Horsman MR, Jackson $A$, Jayson $G$, Judson

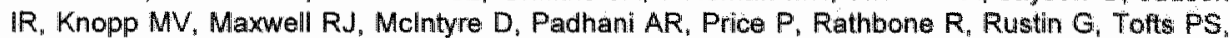
Tozer $\mathrm{GM}_{n}$ Vennart $W$, Waterton JC, Williams SR, Workman $P$. Assessment of antiangiogenic and antivascular therapeutics using MRI: recommendations for appropriate methodology for clinical trials. Br J Radiol. 2003;76 Spec No 1:S87-91.

7. Padhani AR. Dynamic contrast-enhanced MRI in clinical oncology: current status and future directions. J Magn Reson Imaging. 2002;16:407-22.

8. Daldrup HE, Shames DM, Husseini W, Wendland MF, Okuhata $Y$, Brasch RC. Quantification of the extraction fraction for gadopentetate across breast cancer capillaries. Magn Reson Med. $1998 ; 40: 537-43$.

9. van Dijke CF, Brasch RC, Roberts TP, Weidner N, Mathur A, Shames DM, Mann JS, Demsar F, Lang P. Schwickert HC. Mammary carcinoma model: correlation of macromolecular contrastenhanced MR limaging characterizations of tumor microvasculature and histologic capillary density. Radiology. 1996;198:813-8.

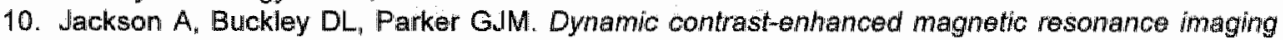
in oncology. 1 ed. Heidelberg, Germany: Springer-Verlag; 2005.

11. Carmeliet $P$. Mechanisms of angiogenesis and arteriogenesis. Nat Med. 2000;6:389-95.

12. van Dijke CF, Kirk BA, Peterfy CG, Genant HK, Brasch RC, Kapila S. Arthritic temporomandibular joint: correlation of macromalecular contrast-enhanced MR imaging parameters and histopathologic findings. Radiology. 1997;204:825-32.

13. Helbich TH, Roberts TP, Rollins MD, Shames DM, Turetschek K, Hopf HW, Muhler M, Hunt TK, Brasch RC. Nonimvasive assessment of wound-healing angiogenesis with contrast-enhanced MRI. Acad Radiol. 2002;9 Suppl 1:S1:45-7.

14. Griffioen AW, Molema $G$. Angiogenesis: potentials for pharmacologic intervention in the treatment of cancer, cardiovascular diseases, and chronic inflammation. Pharmacol Rov. 2000,52:237-68.

15. Post MJ, Simons M. The rational phase of therapeutic angiogenesis. Minenva Cardioangiol. 2003:51:421-32.

16. Wahlberg $E$. Angiogenesis and arteriogenesis in limb ischemia. I Vasc Surg. 2003:38:198-203. 


\section{Objective of this thesis}

The objective of this thesis is to evaluate the use of different MR contrast agents for determining microcirculation characteristics by DCE-MRI in oncology, and to determine the potential of DCE-MRI for determining microcirculation characteristics in arterial occlusive disease.

\section{Outline of this thesis}

In Chapter 2, the use of a clinically available low molecular weight MR contrast agent is compared with the use of a high molecular weight MR contrast agent, for evaluating microcirculation properties in a mouse tumor model, with histological correlation.

In Chapter 3 , the relationship between the molecular weight of MR contrast agents and dynamic contrast-enhanced MR imaging derived parameters were determined, by using different generations of dendritic MR contrast agents.

In Chapter 4, the use of a clinically available low molecular weight MR contrast agent was investigated for evaluating microcirculation changes after anti-vascular anti-tumor treatment in a mouse tumor model.

In Chapter 5 , the use of a clinically available low molecular weight MR contrast agent was investigated for evaluating microcirculation changes after radiation therapy in patients with locally advanced rectal cancer.

In Chapter 6, serial dynamic contrast-enhanced MR imaging and MR angiography was performed to monitor microcirculation characteristics and collateral vessel growth in a rabbit femoral artery ligation model.

In Chapter 7, serial MR angiography with X-ray angiography was performed to monitor collateral vessel growth in a rabbit femoral artery ligation model.

In Chapter $B$, different generations of dendritic MR contrast agents were characterized in terms of paramagnetic and pharmacokinetic properties.

Chapter 9 discusses current MR imaging of neo-vascularization and future directions. 


\title{
Chapter 2
}

\section{Gadopentetate dimeglumine versus USPIO for dynamic contrast-enhanced MRI of tumor angiogenesis in human colon carcinoma in mice}

\author{
Radiology, 2003 Nov;229(2):429-38 \\ Quido G. de Lussanet, Walter H. Backes, Arjan W. Griffioen, Jos M.A. van Engelshoven, \\ Regina G.H. Beets-Tan.
}

\section{ABSTRACT}

\section{PURPOSE}

To compare the kinetic physiologic properties of a clinical contrast agent, gadopentetate dimeglumine, with those of ultrasmall superparamagnetic iron oxide (USPIO) particles for dynamic contrast material-enhanced magnetic resonance (MR) imaging of tumor angiogenesis in human colon carcinoma in mice with a clinical MR imaging unit.

\section{MATERIALS AND METHODS}

Thirty-two mice with human colon carcinoma were injected with either gadopentetate dimeglumine $(n=16)$ or USPIO $(n=16)$ for dynamic contrast-enhanced MR imaging. Dynamic contrast-enhanced MR imaging measurements were analyzed by using a twocompartment model to calculate the endothelial transfer coefficient surface area product $\left(K^{\mathrm{PS}}\right)$ for the tumor microvasculature, the reflux coefficient $(k)$, and the fractional plasma volume $\left(f^{P V}\right) . K^{P S}, k$, and $\mathrm{f}^{\mathrm{PV}}$ maps were compared with histologic microvessel density (MVD) and used to observe differences between core and rim regions of tumor.

\section{RESULTS}

Results in 30 mice (15 in the gadopentetate dimeglumine group and 15 in the USPIO group) could be used. $K^{P S}$ values measured with both agents correlated well with MVD in hot spots (gadopentetate dimeglumine: $r=0.6, P=.02$; USPIO: $r=0.6, P=.01$ ). No significant difference $(P=.4)$ in correlation was found between the two agents. Both USPIO and gadopentetate dimeglumine demonstrated higher MVD and $K^{\text {PS }}$ values in tumor rim than in tumor core $(P<.01)$. Tumor $k$ values correlated poorly with wholetumor MVD for both gadopentetate dimeglumine $(r=0.3, P=4)$ and USPIO $(r=0.2, P=$ .6). while $f^{p V}$ values correlated well with whole-tumor MVD for USPIO $(r=0.6, P=.02)$ but not gadopentetate dimeglumine $(r=-0.01, P=.98)$.

\section{CONCLUSION}

The kinetic physiologic properties of gadopentetate dimeglumine are as good as those of USPIO for dynamic contrast-enhanced MR imaging for calculating $K^{\text {pss }}$ as a measurement of angiogenesis in human colon carcinoma. Further studies with patients may reveal whether gadopentetate dimeglumine might be used for this purpose in clinical practice. 


\section{Introduction}

Tumor growth and metastasis formation are dependent on angiogenesis, the process of new blood vessel formation (1). Modulation of angiogenesis is a promising strategy for tumor treatment $(1,2)$. To gain insight into the angiogenic potential of a tumor, new methods for diagnosis have been developed worldwide, among which noninvasive imaging techniques are promising. Dynamic contrast material-enhanced $T 1$-weighted magnetic resonance (MR) imaging is a noninvasive technique that can aid in the diagnosis of angiogenic activity by enabling the measurement of increased microvessel permeability and blood volume in the voxels in a selected volume of angiogenic tissue $(3.4)$. Dynamic contrast-enhanced MR imaging enables the measurement of microvessel permeability by permitting assessment of the rate at which a contrast agent transfers from the blood to the extravascular extracellular space. This rate can be expressed as the endothelial transfer coefficient surface area product $\left(K^{\mathrm{PS}}\right)$. Similarly, the transfer of the contrast agent back to the blood can be expressed as the reflux coefficient $(k)$. In addition, dynamic contrast-enhanced MR imaging enables the measurement of increased blood volume by permitting the calculation of fractional plasma volume (f ${ }^{\text {vid }}$ ) (5).

Reports in current literature suggest that the suitability of dynamic contrast-enhanced MR imaging is largely dependent on the choice of contrast agient (6). Clinically approved low molecular weight contrast agents, such as gadopentetate dimeglumine $(7)$, are thought to be less suited for dynamic contrast-enhanced MR imaging than largemolecular contrast agents $(5,6)$, such as so-called ultrasmall superparamagnetic iron oxide (USPIO) particles (8). Gadopentetate dimeglumine has ideal paramagnetic properties for highly $\mathrm{T} 1$-weighted MR imaging $(9,10)$. However, its short circulation times and fast diffusion rate compared with the blood pool characteristics of USPIO are drawbacks (11). To date, USPIO particles are not clinically approved for use in dynamic contrast-enhanced MR imaging (12). USPIO particles also induce a relatively strong T2* reduction, which may affect T1-weighted MR imaging $(13,14)$. However, the T2* effect could be useful for the assessment of tissue angiogenesis when one is performing $T 2^{*}$ weighted measurements (15).

To our knowledge, to date there have been no published reports of studies in which the MR imaging-derived microcirculation characteristics of clinically approved low molecular weight contrast agents were directly compared with those of USPIO particles in the depiction of tumor angiogenesis. The most commonly used and appropriate histologic validation technique for dynamic contrast-enhanced MR imaging results is the determination of the microvessel density (MVD) in stained tumor specimens $(3,6,16)$.

Thus, the purpose of this study was to compare the kinetic physiologic properties of gadopentetate dimeglumine with those of USPIO particles for dynamic contrastenhanced MR imaging of tumor anglogenesis in human colon carcinoma in mice with a clinical MR imaging unit.

\section{Materials and Methods}

\section{Animal model}

This study was approved by the ethical review committee of our inistitution. Thirty-two imale nude mice (Swiss nu/nu, Charles River, Maastricht, the Netherlands) (age range, 6-10 weeks) received an injection) of $10^{6} \mathrm{LS} 174 \mathrm{~T}$ human colon carcinoma cells 
subcutaneously in the left flank. The mice were randomly selected for imaging with either gadopentetate dimeglumine or USPIO particles. To increase variation in tumor volume and MVD, the mice were also selected to bie imaged on different days after tumor injection (i.e., on day 10 , day 13 , day 16 , or day 19 ).

Before imaging, the mice were anesthetized with subcutaneous injection of $100 \mathrm{mg}$ of ketamine (Nimatek; Eurovet, Bladel, the Netherlands) per kilogram of body weight and $10 \mathrm{mg} / \mathrm{kg}$ of xylazine $\mathrm{HCl}$ (Sedamun; Eurovet). The temperature in the MR imaging unit bore was kept at approximately $28^{\circ} \mathrm{C}$ by placing warm water bags near the mouse. For contrast agent injection into the tail vein, a $50-\mathrm{cm} \times 0.28-\mathrm{mm}$ (inner diameter) tube (nonsterile tubing, Portex, Hythe. England) with a 27 -gauge needle tip was prepared with three fluid volumes: an anticoagulant $(0.5 \mu \mathrm{L}$ of 0.5 moll $\mathrm{L}$ heparin [Heparine; Leo Pharma. Breda, the Netherlands]); either $0.1 \mathrm{mmo} / \mathrm{kg}$ of gadopentetate dimeglumine (Magnevist; Schering, Berlin, Germany) or, in the manner advised by Turetschek et al $(8,17), 2.5 \mathrm{mg} F \mathrm{Fe} / \mathrm{kg}$ of USPIO particles (NC100150 injection for biologic use only lbatch no. 812010], Nycomed Imaging, Oslo, Norway); and $15 \mu \mathrm{L}$ of saline $(0.3 \mathrm{~mol} / \mathrm{L} \mathrm{NaCl})$ to flush the bolus. The contrast agent and saline were manually injected during the fifth dynamic volume acquisition in less than 40 seconds. Hematocrit values were determined from centrifuged glass heparinized capillary tubes (Micro-Hematocrit Capillary Tubes; Chase Scientific Glass, Rockwood, Tenn) of $75 \mathrm{~mm}$ in length and $1.1 \mathrm{~mm}$ in inner diameter that were filled with blood from an eye orbit puncture performed immediately after MR imaging to relate blood and plasma tracer concentrations.

\section{MR' contrast agents}

Gadopentetate dimeglumine is a small $(0.5 \mathrm{kDa},<1 \mathrm{~nm})$ paramagnetic gadolinium chelate with non-protein-interacting and extracellular properties. After intravenous injection, plasma concentrations of this agent decline biexponentially with time owing to (a) rapid diffusion to the extracellular space (distribution phase half-life: 10 minutes) and (b) renal clearance (elimination phase half-life: 100 minutes) $(18,19)$. Gadopentetate dimeglumine is approved for use worldwide and is used for many clinical MR imaging applications (20).

USPIO is a superparamagnetic iron oxide crystal $\left(\mathrm{Fe}_{3} \mathrm{O}_{4}\right)$ with an oxidized starch coating (particle size, $<20 \mathrm{~nm}$ ). USPIO particles, in general, are near clinical approval, and NC100150 injection has been used in phase II trials for liver imaging (12) and MR anglography (21). After intravenous injection, USPIO particles reduce the T1 relaxation time of blood to below $100 \mathrm{msec}$ for more than 2 hours before they are taken up mainly by the liver reticuloendothelial system (half-life: 45 minutes) $(22,23)$.

\section{MR imaging}

MR imaging was performed with a small (5-cm-diameter) surface coil and a 1.5-T MR imaging system (Philips Intera; Philips Medical Systems, Best, the Netherlands). A T2. weighted anatomic acquisition (multisection turbo spin echo; repetition time mseclecho time msec, 3,300/200; flip angle, $90^{\circ}$ ) that covered the tail-end half of the mouse was performed for tumor delineation and size measurements and to yield an anatomic reference for the subsequent acquisitions. Before and 60 minutes after contrast agent administration, T2-weighted (multisection turbo spin echo; 300/25, 50; flip angle, 90 and $\mathrm{T} 22^{*}$-weighted (three-dimensional fast field echo; 70/25, 50; flip angle, $15^{\circ}$ ) dual-echo acquisitions were performed, with 16 transwerse sections, matrix dimensions of $128 \times$ 128 , and a woxel size of $0.5 \times 0.5 \times 2.0 \mathrm{~mm}^{3}$. Six T1-weighted acquisitions (threedimensional fast field echo, 50/7) with different flip angles $\left(2^{\circ}, 5^{\circ}, 10^{\circ}, 15^{\circ}, 25^{\circ}\right.$, and $\left.35^{\circ}\right)$ 
wer performed so that we could determine the local T1 relaxation times before contrast enhancement (24). The T1-weighted dynamic contrast-enhanced series (76 volume acquisitions; temporal resolution, 39 seconds) were performed with the same parameters as the $\mathrm{T} 1$ relaxation measurements (flip angle, $35^{\circ}$ ). The spatial resolution of the acquired sections was $0.5 \times 0.5 \times 4 \mathrm{~mm}^{3}$. Adjacent sampled sections were $2-\mathrm{mm}$ displaced and subsequently interpolated to 2.0 - mm-thick sections during reconstruction.

\section{Analysis of MR imaging data}

The T2-weighted anatomic acquisition was used to locate and delineate the tumor. The volume of each tumor was measured. This was achieved by determining the areas (in square millimeters) of the tumor in consecutive MR sections that contained tumor tissue. The sum of these areas was multiplied by the section thickness $(2 \mathrm{~mm})$. The kinetic analysis of the images is described briefly in the Appendix. The data processing was performed in the MATLAB programming environment (The MathWorks, Natick, Mass). Local time-averaged $T 1$ relaxation rates (ie, $R 1_{0}$ ) of the precontrast images and the postcontrast image signal intensity time courses (with signal intensity measured in arbitrary units [AU]) were used to determine the plasma concentration in the aorta, representing the arterial input function $\mathrm{R} t(t)$ and the tissue concentration in the tumor. Individual R1 $(t)$ time courses were used to correct for variations in manual injection of the contrast agent, intrinsic circulation properties, and contrast agent elimination.

$K^{P S}, k$, and $f^{P V}$ values were determined by applying a two-compartment bidirectional model $(5,25)$. The $K^{P S}$ and $k$ values were expressed as milliliters per minute per $100 \mathrm{~cm}^{3}$ of tisisue, and the $f^{p V}$ values were expressed as milliliters per cubic centimeter of tissue. The plasma relaxation time course for the arterial input function of gadopentetate dimeglumine was fitted to a biexponential function to account for the fast extracellular distribution and slow renal clearance of this agent. Conversely, the USPIO plasma relaxation time course could be modeled with a monoexponential decay function. The relationship between the T1 shortening of the signal enhancement and the contrast agent concentration was determined from the analyses of in vitro concentration measurements for both agents. For USPIO, which is known to exhibit relatively large T2* shortening, the $\mathrm{T}^{*}$ effects were included in this relationship as described by Tofts et al (26), again by using the analyses of the in vitro measurements. We assumed a linear relationship between changes in relaxation rate and concentration for both agents.

The kinetic analyses were performed for each voxel in the central section through the tumor. These analyses yielded color-coded maps of the $k^{\mathrm{PS}}$ values, which were overlaid on the corresponding precontrast T1-weighted images. These $K^{p s i}$ maps were then used to define regions of interest for the whole tumor, the tumor rim, and the tumor core so that we could spatially average $K^{P S}, k_{i}$ and $f^{P V}$ values for these three tumor regions. Preand postcontrast $T 2$ and $T 2^{*}$ values were determined by drawing regions of interest for the whole tumor, tumor rim, and tumor core on the dual-echo images of corresponding sections, as performed for the kinetic analysis. The areas of whole-tumor regions of interest (range, $6-140 \mathrm{~mm}^{2}$ ) varied depending on the size of the individual tumor. The dual-echo signal intensity values in these regions were used to calculate the $T 2$ and $T 2^{*}$ values by dividing 25 insec (ie the first echo time minus the second echo time) by the natural logarithm of the signal intensity ratio. Finally, the pre- and postcontrast T2 and $T 2^{*}$ values were inverted to calculate the changes in $T 2$ and $T 2^{*}$ relaxation rates (ie, $R 2$ and $\mathrm{R}^{*}{ }^{*}$, respectively) after contrast agent injection. 


\section{Histological examination}

After MR imaging, the mice, while still anesthetized, were sacrificed by means of cervical dislocation, and the tumors were excised. Transverse cryosections ( $4 \mu \mathrm{m})$ were obtained through the middle part of the tumors, corresponding to the transverse orientation of the selected MR imaging sections. Skin tissue marked the lateral side of the tumors on the cryosections. The cryasections were immunohistochemically stained by using CD31 antibody (Goat-anti-Rat-PO-DAB; Pharmingen, Uithoorn, the Netherlands).

MVD values were calculated from the total number of vessels in a microscopic field of $0.2 \mathrm{~mm}^{2}$ (magnification, $\times 250$ ) for the whole cryosection through the tumor, creating a MVD map for each cryosection. The method of counting vessels in whole cryosections through the tumor, instead of in only random fields or vascular hot spot selections, was chosen to reduce operator dependency. MVD values were abtained independently from the MR imaging analyses. MVD values were calculated for the whole tumor, the tumor rim, the tumor core, and microvessell hot spots (ie, the five highest MVD values per tumor cryosection). Testing for a correlation between $K^{P S}$ and histologic tumor grade, a correlation found, for instance, in rat breast tumors (5), is not applicable in this xenograft model. This is because all tumors in this model are intermediately to well differentiated and hardly ever metastasize, implying limited variation in tumor grade.

\section{In vitro experiment}

To estimate the T2* effect of USPIO $(8,17)$, we used commercially obtained mouse plasma (Harlan SeraLab, Loughborough, England) with citrate buffer. We prepared 21 samples of $2.5 \mathrm{~mL}$ : 10 samples with increments in concentration of gadopentetate dimeglumine (range, $0.01-0.50 \mathrm{mmol} / \mathrm{mL}$ of plasma), 10 samples with increments in concentration of USPIO (range, $0.001-5.000 \mathrm{mg}$ Fe per milliliter of plasma), and one control plasma sample. The same MR imaging protocol (T1 measurement and T2 and $\mathrm{T} 2^{*}$ dual-echo measurements) and equipment were used as in the mouse experiment. The slopes of the $T 1, T 2$, and $T 2^{*}$ rellaxations versus the contrast agent concentrations. in plasma ( $r 1_{\text {plasma, }} r 2_{\text {plasma, }}$ and $r 2^{*}$ plasma $)$ were calculated, and the ratios $r 2_{\text {plasma }} / r_{\text {plasma }}$ and $r 2^{*}$ plasma $/ r{ }^{1}$ plasma were determined for both gladopentetate dimeglumine and USPIO.

\section{Statistical analysis}

The number of animals included were estimated by performing a power analysis (27) $(95 \% \mathrm{Cl}$ and $80 \%$ power) and considering a predetermined possible dropout rate of less. than $10 \%$, which amounted to 16 animals per contrast agent group, for a total of 32 animals. MVD values were compared with tumor volumes, $K^{p s}, k_{*}$ and $f^{p V}$ values, and change in R2 and R2* values, and tumor ages (in terms of days after inoculation) were compared with tumor volumes by determining the Spearman rank correlation coefficlent. The Spearman rank correlation allows statistical inference from a non-normal distribution of variables. The equality of the $K^{P S}$ correllation coefficients of the gadopentetate dimeglumine and USPIO groups was tested by using Fischer $z$ transformation (27).

MVD values and tumor volumes were compared between the gadopentetate dimeglumine and USPIO groups by using the two-tailed Student $t$ test. The two-tailed Student $t$ test was also used for comparing reductions in tumor $\mathrm{R} 2$ and $\mathrm{R} 2^{\text {k }}$ values after contrast agent injection within the gadopentetate dimeglumine and USPIO groups; for comparing differences in MVD, $K^{\mathrm{PS}}, K_{1}$ and $\mathrm{fV}^{\mathrm{PV}}$ values and change in R2 and $\mathrm{R} 2{ }^{*}$ between tumor rim and tumor core; and for comparing $T 2^{*}$ signal variations in tumor rim with $T 2^{*}$ signal deviations in tumor core and muscle. Statistical analyses were performed by using commercial software (SPSS 10.0.5; SPSS, Chicago, 111 ), and $P<.05$ was considered to indicate a statistically significant difference. 


\section{Results}

Thirty mice were successfully imaged (one died and one could not be successfully imaged owing to severe motion artifacts) and included in the evaluation of MVD measurements.

\section{Tumor charactenstics}

Tumor volumes ranged between 25 and $1,500 \mathrm{~mm}^{3}$ (average volume, $443 \mathrm{~mm}^{3} \pm 539$ [SD] with USPIO and $495 \mathrm{~mm}^{3} \pm 445$ with gadopentetate dimeglumine) and showed a negative correlation with MVD $(r=-0.57, P<.05)$ and a positive correlation with tumor age $(r=0.40, P<.05)$ (Table 1). The MVD ranged between 3.1 and 20.5 vessels per 0.2 $\mathrm{mm}^{2}$ (average MVD $7.1 \pm 5.3$ with USPIO and $5.0 \pm 2.2$ with gadopentetate dimeglumine). Standard error of the mean for individual MVD values ranged between $5 \%$ and $15 \%$ and was slightly higher for rim MVD values than for core MVD values. Neither the mean tumor volume $(P=.9)$ nor the mean MVD value $(P=.2)$ was significantly different between the USPIO and gadopentetate dimeglumine groups. The mean MVD value in the tumor rim was significantly $(P<.01)$ higher than that in the tumor core (Table 1). The mean measured hematocrit value was $0.48 \pm 0.03$.

\section{Dynamic T1-weighted measurements}

Quantitatively color-coded $K^{\mathrm{PS}}$ maps and their corresponding T2-weighted images are shown in Figure 1. These maps highlight the areas of high angiogenic activity and show the possible variation between tumors. In 22 of the 30 tumors, more than $90 \%$ of the pixels containing tumor tissue were successfully analyzed; in four tumors (one imaged with gadopentetate dimeglumine, three imaged with USPIO) about $25 \%$ of the pixels were rejected; and in four tumors (two imaged with gadopentetate dimeglumine, two imaged with USPIO), about $50 \%$ of the pixels were rejected owing to nonenhancement or because a preset maximum fit error, as described in the Appendix, was exceeded.

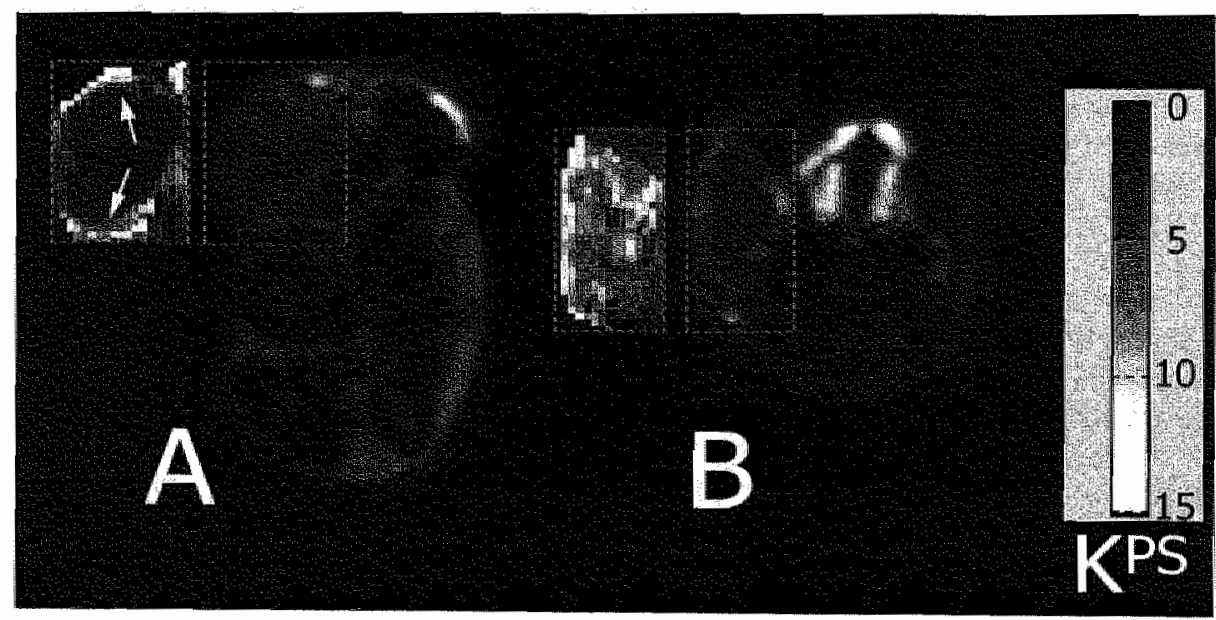

Figure 1 . The color** coded tumor $K^{P S}$ maps and corresponding axial $T_{2}$-weighted anatomic image of mice with (a) a tumor with a high $K^{\text {PS }}$ at the rim (arrow) with respect to the tumor core and (b) a heterogeneous tumor. * * Color image is on back-cover (middle). 
Table 1. Tumar characteristics and DC MRI derived parameters by ascending MVD

\begin{tabular}{|c|c|c|c|c|c|c|c|c|c|}
\hline \multicolumn{10}{|c|}{ Gadiopentetate dimeglumine } \\
\hline Age & Vollume & \multicolumn{2}{|c|}{ MVD } & \multicolumn{2}{|c|}{$k^{\mathrm{es}}$} & \multicolumn{2}{|c|}{ k } & \multicolumn{2}{|c|}{$f^{4}$} \\
\hline & & $\mathrm{rim}$ & core & rim & core & rìm & core & rim & $\operatorname{con}$ \\
\hline 16 & 898 & 2.8 & 2.5 & 3.6 & 10 & 11.2 & 4.0 & 45 & 00 \\
\hline 13 & 25 & 3.7 & 3.7 & 4.7 & 4.7 & 9.9 & 9.9 & 7.5 & 7.5 \\
\hline 19 & 327 & 4.0 & 3.1 & 5.2 & 54 & 16.4 & 23.6 & 7.7 & 3.1 \\
\hline 19 & 521 & 4.2 & 3.8 & 4.8 & 4.1 & 231 & 22.6 & 5.9 & 1.2 \\
\hline 16 & 694 & 4.9 & 2.7 & 4.6 & 1.1 & 2.6 & 0.6 & 3.9 & 0.1 \\
\hline 16 & 814 & 5.7 & 2.7 & 6.7 & 0.5 & 6.4 & 6.0 & 8.9 & 0.4 \\
\hline 19 & 1688 & 5.8 & 2.4 & 4.6 & 2.0 & 11.3 & 3.5 & 9.6 & 4.9 \\
\hline 16 & 58 & 5.8 & 25 & 5.0 & 9.1 & 0.6 & 0.0 & 1.4 & 1.3 \\
\hline 10 & 277 & 5.9 & 2.7 & 4.5 & 2.6 & 6.1 & 2.5 & 12.7 & 2.2 \\
\hline 19 & 881 & 6.2 & 5.8 & 7.0 & 3.4 & 17.1 & 14.2 & 42,0 & 20.8 \\
\hline 19 & 281 & 7.1 & 4.3 & 4.3 & 3.8 & 0.0 & 0.0 & 0.0 & 0.0 \\
\hline 19 & 341 & 7.4 & 5.6 & 8.7 & 6.7 & 33.4 & 38.4 & 0.9 & 0.0 \\
\hline 10 & 420 & 7.5 & 7.9 & 5.4 & 26 & 45.8 & 60.1 & 5.1 & 4.7 \\
\hline 13 & 178 & 8.7 & 4.6 & 7.0 & 5.1 & 7.3 & 0.0 & 0.0 & 0.0 \\
\hline 10 & 32 & 11.7 & 12.1 & 7.4 & 8.8 & 33.4 & 34.5 & 15.4 & 13.3 \\
\hline $16+4$ & $495+445$ & $64+22$ & $4.4 \pm 2.6$ & $5.6+1.4$ & $3.3+2.4$ & $15 \pm 14$ & $15 \pm 18$ & $8+10$ & $4 \pm 16$ \\
\hline $\mathrm{rim} / \mathrm{cor}$ & difference: & $28 \%$ & & 43 & & $1 \%$ & & & \\
\hline \multicolumn{10}{|c|}{ USPIO } \\
\hline Age & Vollume & \multicolumn{2}{|c|}{ MVD } & \multicolumn{2}{|c|}{$K^{P S}$} & \multicolumn{2}{|c|}{$k$} & \multicolumn{2}{|c|}{$f^{p v}$} \\
\hline & & rim & core & rim & core & rim & core & $\mathrm{rim}$ & colro \\
\hline 19 & 1617 & 3.8 & 2.6 & 4.4 & 2.6 & 0.1 & 0.0 & 4.1 & 0.0 \\
\hline 16 & 238 & 3.8 & 3.3 & 11.5 & 5.4 & 1.2 & 0.2 & 32.3 & 0.0 \\
\hline 19 & 1612 & 4.4 & 4.0 & 5.8 & 31 & 1.3 & 0.0 & 2.3 & 0.0 \\
\hline 19 & 60 & 4.6 & 1.9 & 6.4 & 5.7 & 0.3 & 0.0 & 25.1 & 3.2 \\
\hline 16 & 392 & 5.1 & 2.8 & 5.2 & 1.6 & 1.6 & 0.0 & 25.2 & 0.0 \\
\hline 19 & 217 & 5.7 & 2.3 & 3.4 & 2.5 & 0.1 & 0.0 & 7.1 & 0.3 \\
\hline 19 & 1046 & 6.0 & 28 & 5.9 & 2.1 & 1.6 & 0.0 & 34.9 & 0.5 \\
\hline 13 & 262 & 6.2 & 1.2 & 3.4 & 1.7 & 2.1 & 1.4 & 2.3 & 0.0 \\
\hline 16 & 424 & 6.9 & 4.7 & 5.1 & 2.9 & 2.1 & 0.7 & 49.7 & 16.0 \\
\hline 13 & 334 & 10.5 & 5.1 & 9.4 & 7.4 & 3.2 & 0.0 & 26.5 & 10.2 \\
\hline 10 & 26 & 10.8 & 6.2 & 14.6 & 14.6 & 0.9 & 2.1 & 100.0 & 900.0 \\
\hline 13 & 58 & 11.0 & 9.5 & 13.6 & 15.0 & 0.1 & 0.0 & 73.0 & 100.0 \\
\hline 10 & 256 & 11.6 & 9.2 & 12.5 & 12.5 & 0.0 & 0.0 & 3.6 & 0.5 \\
\hline 10 & 86 & 16.2 & 16.3 & 12.7 & 12.7 & 0.2 & 0.0 & 11.4 & 32.8 \\
\hline 10 & 25 & 20.5 & 20.5 & 13.2 & 13.2 & 1.3 & 1.3 & 43.5 & 43.5 \\
\hline \multirow{2}{*}{\multicolumn{2}{|c|}{ rim/ core difference: }} & 8. $5+4.9$ & $6.2+5.6$ & $8.5+4.1$ & $6.9+5.2$ & $1.1 \pm 0.9$ & 0.440 .7 & 29128 & 21335 \\
\hline & & $34 \%$ & & \multicolumn{2}{|c|}{$28 \%$} & \multicolumn{2}{|c|}{$64 \%$} & \multicolumn{2}{|c|}{$30 \%$} \\
\hline
\end{tabular}

\footnotetext{
Note: Age is the number of days after inoculation: MVD is the number af vessels per $0.2 \mathrm{~mm}^{2_{2}}$ Volume is measured with MRI in $\mathrm{mm}^{3}$; percentages (\%) are the differences between rim and core values relative to the core values, all are significant $\left(p<0.01^{\text {if }}\right.$ and $p=0.03^{\text {b }}$ ) except for Gd-DTPA $k$ values $\left(p=0.9^{\circ}\right)$.
}

The $K^{P S}$ values for the USPIO group (mean, $8.1 \pm 4.3$ [SD]) were significantly higher ( $P=$ .01) than for the gadopentetate dimeglumine group (mean, 4.6 1.6). The two agents had similar correlation coefficients (Table 2) but yielded different scatterplots (Figure 2), which limits the prediction of MVD from $K^{P S}$, especially at high MVD values with gadopentetate dimeglumine. Nevertheless, the $K^{\text {fs }}$ values measured with either agent correlated significantly with MVD. The differences between the correlation coefficients for the gadopentetate dimeglumine and USPIO groups were not significant $(P>4)$. The $K^{p s}$ generally correlated less significantly with MVD in hot spots than with MVD in the whole tumor and in the tumor rim (Table 2). 
Table 2. Statistical correlation between MVD and the DC MRI parameters

\begin{tabular}{|c|c|c|c|c|c|c|}
\hline \multirow{2}{*}{\multicolumn{2}{|c|}{ MRI parameter }} & & \multicolumn{2}{|c|}{ Gd-DTPA } & \multicolumn{2}{|c|}{ USPIO } \\
\hline & & MVD & Spearman, $r$ & $\mathbf{p}$ & Spearman, $r$ & $\mathrm{p}$ \\
\hline \multirow{5}{*}{$\kappa^{\circ}$} & tumor & hatspot & 0.6 & 0.02 & 0.6 & 0.01 \\
\hline & rim & hotspot & 0.6 & 0.02 & 0.5 & 0.05 \\
\hline & tumor & tumor & 0.7 & 0.006 & 0.8 & 0.001 \\
\hline & rim & rim & 0.6 & 0.03 & 0.6 & 0.02 \\
\hline & core & core & 0.7 & 0.004 & 0.8 & 0.001 \\
\hline \multirow[t]{3}{*}{$k$} & tumar & tumar & 0.3 & 0.4 & 0.2 & 0.6 \\
\hline & rim & rim & 0.2 & 0.4 & -0.1 & 0.7 \\
\hline & core & core & 0.6 & 0.03 & -0.03 & 0.9 \\
\hline \multirow[t]{3}{*}{$f^{p v}$} & tumor & tumor & -0.01 & 1.0 & 0.6 & 0.02 \\
\hline & rim & rim & -0.1 & 0.8 & 0.3 & 0.3 \\
\hline & core & care & 0.2 & 0.5 & 0.7 & 0.004 \\
\hline
\end{tabular}

Note: MVD is the microvessel density (vessels per $\left.0.2 \mathrm{~mm}^{2}\right) ; K^{P S}$ is the microvessel permeability surface area product $\left(\mathrm{ml} \mathrm{min}^{-1} 100 \mathrm{~cm}^{-3}\right.$ of tissue); $k$ is the reflux coefficient $\left(\mathrm{ml} \mathrm{min} \mathrm{min}^{-1} 100 \mathrm{~cm}^{-3}\right.$ of tissue); $\mathrm{f}^{\mathrm{PV}}=$ fractional plasma volume $(\mathrm{ml} \mathrm{cm}$ of tissue).

Contrary to Turetschek et al (8), we could derive the $k$ values from the data by using the bidirectional model and did not set the $k$ at zero. However, the $k$ values measured with USPIO were very small (mean, $0.9 \pm 0.8)-15$ times smaller $(P=.003$ ) than the values measured with gadopentetate dimeglumine (mean, 14.0 15.0 ). The $k$ vallues measured with USPIO showed no correlation with MVD. Measurements with gadopentetate dimeglumine showed a positive correlation between $k$ and MVD that was significant for the tumor core only (Table 2). The $f^{p v}$ values differed between the two agents. The tumor $f^{p v}$ values measured with USPIO (mean, $0.20 \pm 0.30$ ) were three to four times higher $(P=.04)$ than those measured with gadopentetate dimeglumine (mean, $0.07 \pm$ 0.09). The $f^{P V}$ significantly correlated with MVD when measured with USPIO (except in the tumor rim) but not when measured with gadopentetate dimeglumine (Table 2).

Additional dynamic contrast-enhanced MR imaging results revealed that, like the MVD values, all $K^{P S}, f^{p V}$, and $k$ values-with the exception of $k$ values measured with gadopentetate dimeglumine-were significantly higher in the tumor rim than in the tumor core Table 1.

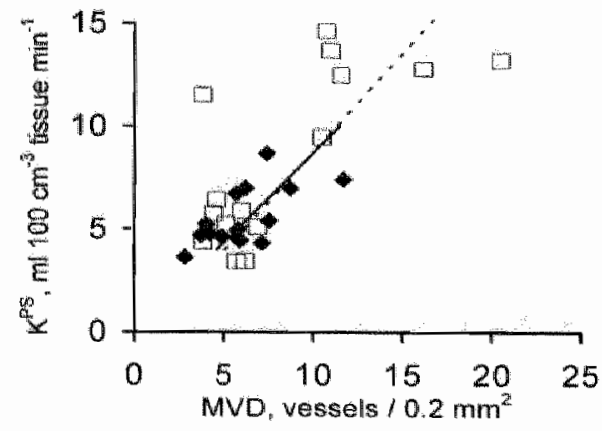

Flgure 2. $K^{\mathrm{PS}}$ tumor rim versus MVD tumor rim scatter plots show a smaller range in MVD for Gd-DTPA (-) than for USPIO (D); regression lines illustrate that correlation between $K^{P S}$ and MVD values for Gd-DTPA (_ do not significantly differ $(p>0.4)$. 


\section{Signal intensity time courses}

Differences in the magnetic properties of the agents were illustrated by their plasma and tissue concentration time courses (Figure 3). The USPIO plasma signal intensity time course showed an immediate signal intensity decrease followed by a slow recovery (Figure 3a). Conversely, the gadopentetate dimeglumine signal intensity time course showed an immediate signal intensity increase followed by a decay (Figure 3b). The signal intensity of tumor tissue showed very small increases with USPIO (Figure 3 a) as compared with gadopentetate dimeglumine, which caused a relatively large increase in signal intensity that was followed by a slow reduction starting approximately 15 minutes after injection (Figure 3b). Small areas in the rims of most tumors showed decreases in signal intensity over time that resembled the signal intensity time course of plasma, indicating that these areas had a strong vascular content. With USPIO, these appeared as small areas of strong signal intensity reduction in the fumor rim on the dynamic images obtained immediately after contrast agent injection.
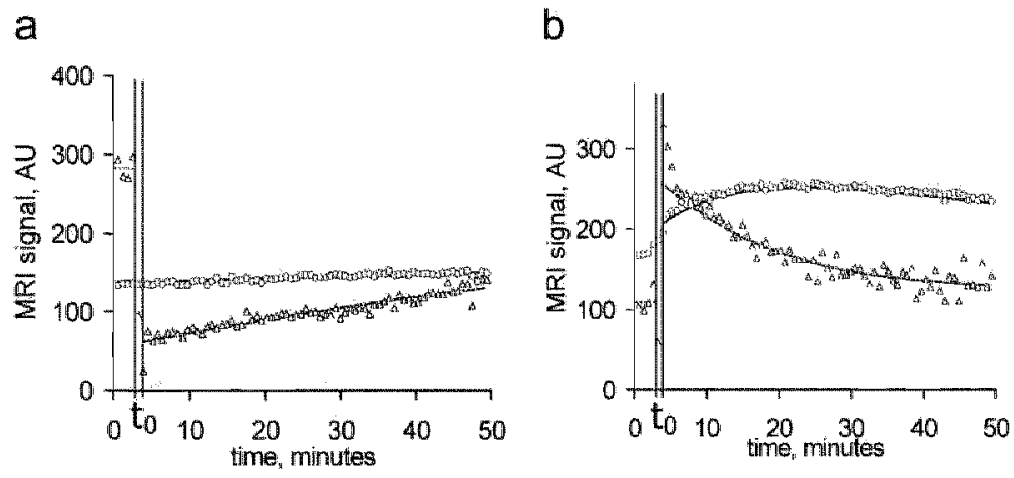

Figure 3. (a) USPIO plasma signal time course, as measured in the arta $(\Delta)_{\text {, }}$ shows an immediate signal drop after contrast injection $\left(t_{0}\right)$ followed by a slow recovery $(\ldots . . . .$.$) , and the average signal$ time course measured in the tumor $(O)$ follows a small but gradual increase $(-)$ after contrast injection $\left(t_{0}\right)$.

(b) Gd-DTPA plasma signal time course, as measured in the aorta $(\Delta)$, shows an immediate signal

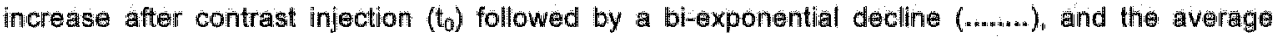
signal time course measured in the tumor (O) showed a substantial signal increase which diminishes again ( $(-)$ ) after approximately 15 min post injection (to).

\section{T2 and T2* Relaxation Rate Measurements}

The $\mathrm{T}_{2}^{*}$ relaxation rates in tumor were approximately $25 \%$ higher with USPIO than with gadopentetate dimeglumine (Figure 4). USPIO caused a significant $10 \%$ R2* reduction (from 39.0 to $43.1 \mathrm{sec}^{-1} ; P=.02$ ) in tumor, while gadopentetate dimeglumine did not (from 35.2 to $34.1 \mathrm{sec}^{-1} ; P=.4$ ). Reductions in R2 were not significant with either USPIO (from 18.5 to $17.2 \mathrm{sec}^{-1} ; P=1$ ) or gadopentetate dimeglumine (from 16.7 to $16.5 \mathrm{sec}^{-1}$; $P=.5$ ). No significant correlation was found between changes in R2 and R2* and MVD with either USPIO $(P>3)$ or gadopentetate dimeglumine $(P>.5)$. However, the relative SDs of the T2 and especially the T2* image signal intensities were more than $45 \%$ at the tumor rim (mean signal intensity, $654 \mathrm{AU} \pm 295$ [SD]). This deviation was significantly higher than that in the tumor core (mean signal intensity, $670 \mathrm{AU} \pm 167$ ) and that in muscle tissue (mean signal intensity, $740 \mathrm{AU} \pm 187$ ) with both agents $(P<.01)$. 


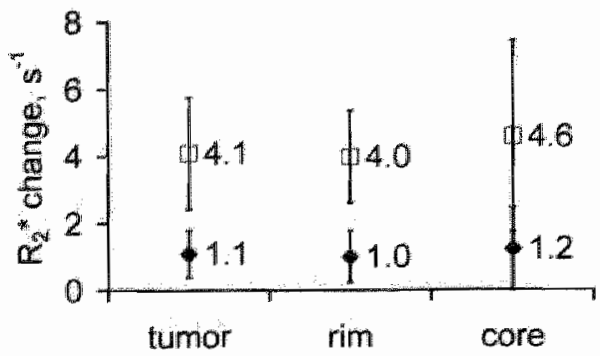

Figure 4. $\mathrm{R}^{*}$ changes in the tumor after contrast agent injection are much greater for USPIO () than for Gd-DTPA (*); error bars represent standard errors of the mean.

\section{In vitro experiment}

The in vitro measurements in our study supported the idea that the assumed linear relations between $T 1, T 2$, and $T 2^{*}$ relaxation rates and concentration were sufficient. The plasma relaxivity values ( $r 1_{\text {plasma }}, 2_{\text {plasma, }}$ and $r 2^{*}$ plasma ) for the concentration ranges lested for gadopentetate dimeglumine and USPIO are given in Table 3 . The $r_{\text {plasma }} / r^{1} 1_{\text {plasmia }}$ (and the $r 2^{*}$ plasma $/ r 1_{\text {plasmal }}$ ) ratio is about three times higher for USPIO than for gadopentetate dimeglumine; this illustrates that USPIO has a smaller signal intensityincreasing effect in the acquired T1-weighted images (Table 3 ) for the echo time used.

Table 3. Plasma relaxation, in vitro

$\begin{array}{lllcc} & \text { control plasma } & & \text { Gd-DTPA } & \text { USPIO } \\ T_{1}(\mathrm{~ms}) & 1596 & r_{11 \text { plasma }}\left(\mathrm{mM}^{-1} \mathrm{~s}^{-1}\right) & 5.6 & 17 \\ T_{8}(\mathrm{~ms}) & 150 & r_{2 \text { plasma }}\left(\mathrm{mM}^{-1} \mathrm{~s}^{-1}\right) & 5.8 & 54 \\ T_{2^{*}}^{*}(\mathrm{~ms}) & 124 & \mathrm{r}_{2 \text { plasma }}\left(\mathrm{mM}^{-1} \mathrm{~s}^{-1}\right) & 7.6 & 58\end{array}$

$r_{2 \text { plasma }} / r_{1 \text { plasma }} \quad 1.0$

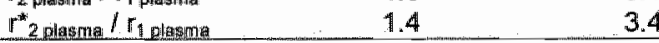

Note: $\quad r_{1}$ plasma $r_{2}$ plasma and $r_{2}^{*}$ plasma are the slopes of $1 / T_{1}, 1 / T_{2}$ and $1 / T_{2}{ }^{*}$ versus contrast agent concentration (C) in pllasma, respectively.

\section{Discussion}

The main finding of this study was that permeability values (ie, $K^{p s}$ values), as measured with dynamic contrast-enhanced MR imaging, showed equal correlation with histologic findings (1e. MVD) when either gadopentetate dimeglumine or USPIO was used (Table 2). To our knowledge, this is the first study that revealed a significant correlation between $K^{5}$ and MVD with gadopentetate dimeglumine when a clinical MR imaging unit and a human colon cancer cell line inoculated in animals were used.

\section{Assessment of tumor microvessel permeability}

The results of this study do not support a general statement that large-molecular contrast agents such as USPIO are favorable to low molecular weight agents such as gadopentetate dimeglumine for assessing tumor microvessel permeability (ie, $K^{P S}$ ) with dynamic contrast-enhanced MR imaging. Favorable properties of USPIO, as compared with the properties of gadopentetate dimeglumine, were considered to be its large molecular size and prolonged circulation times. Results of studies of rat breast tumors that involved the use of albumin-bound gadopentetate dimeglumine (5) suggest that large-molecular agents are better than low molecular weight agents for differentiating between benign and malignant tumors because microvessels in benign tumors are 
almost nonpermeable to large-molecular contrast agents but not to low molecular weight contrast agents.

In malignant tumors such as colorectal cancer, however, permeability is expected to be independent of the molecular size of contrast agents because vessel pores in human collon carcinoma are typically much larger $(400-600 \mathrm{~nm})(28)$ than the diameter of either contrast agent used in our study (gadopentetate dimeglumine, $<1 \mathrm{~nm}$; USPIO, $<20 \mathrm{~nm}$ ). Our results-specifically, the equivalent angles of inclination of the regression line through the $K^{P S}$-versus-MVD scatterplot for USPIO and gadopentetate dimeglumine (Figure 2)-support this expectation. This plot suggests that an increase in MVD yields an increase in $K^{p s}$ that is equal for both contrast agents.

Another advantage of the use of large-molecular agents such as USPIO would be the ability to image at lower temporal resolutions because of the prolonged circulation times of large-molecular compared with low molecular weight agents (29). However, results of studies have shown that, to minimize sampling errors of rapid changes in contrast agent concentration, high temporal resolutions are preferable, particularly for imaging the supplying blood vessels $(30,31)$. Moreover, current MR imaging units alleady allow the use of fast-acquisition dynamic contrast-enhanced imaging techniques that enable firstpass permeability measurements (32).

Our $K^{\text {PS }}$ measurements, obtained with a clinical MR imaging unit, suggest that gadopentetate dimeglumine may be as good as USPIO for dynamic contrast-enhanced MR imaging of tumor angiogenesis. In our opinion, gadopentetate dimeglumine may even be preferable to USPIO for clinical dynamic contrast-enhanced MR imaging measurements because of its safe use and worldwide approval (20), as well as its rapid clearance (19), which allows for short-term follow-up.

\section{T2" effects}

A disadvantage of the use of superparamagnetic contrast agents (eg, USPIO) is that T2* relaxation may negatively affect $T 1$-weighted MR imaging $(17,23)$. These effects are most prominent at higher concentrations. Our in vitro measurements (Table 3) and in vivo $\mathrm{T}_{2}{ }^{*}$-weighted measurements (Figure 4 ) reflect the strong $\mathrm{T} 2{ }^{*}$ relaxation effects of USPIO compared with the effects of gadopentetate dimeglumine for the concentrations used in this study. Significant $T 2^{*}$ effects due to angiogenic heterogeneity of the tissue structures $(15,33)$ (ie, correlation with MVD) were not observed in this study with either USPIO ( $P>3$ ) or gadopentetate dimeglumine $(P>5)$.

One explanation for this may be that postcontrast $R 2$ and $R 2^{*}$ were measured $50-60$ minutes after contrast agent injection, when most of the contrast agent had cleared. Furthermore, the deviations of the signal intensity values in the selected regions of interest were high, particularly in the tumor rim, and this may have obscured changes in $\mathrm{R} 2$ and $\mathrm{R}_{2}{ }^{*}$. Our results show significantly higher relative SDs of the individual $\mathrm{T}^{*}$ signals in the tumor rim than in the tumor core and muscle tissue for both gadopentetate dimeglumine and UISPIO. This suggests that measurements of the T2* signal changes shortly after contrast agent injection may, in fact, be sensitive for different levels of angiogenic activity with both gadopentetate dimeglumine and USPIO.

Our results support the notion that possible negative $\mathrm{T} 2^{\text {* }}$ effects of the contrast agent on the T1-weighted dynamic contrast-enhanced MR imaging measurements should be taken into account, particularly with USPIO. 


\section{Values for $k$ and $f^{2}$}

The $k$ and $f^{\text {PU }}$ measurements reflect differences between USPIO and gadopentetate dimeglumine. The $k$ values illustrate differences in circulation and diffusion properties. The $P^{\text {PV }}$ values illustrate differences in magnetic properties, as well as differences in mobility through the interstitial matrix of the extravascular extracellular space.

The rapid diffusion of gadopentetate dimeglumine causes a rapid decrease in intravascular plasma concentration $(18,19)$, which allows for a higher reflux throughout the tumor. Conversely, the slow clearance of USPIO $(22,23)$ resulted in a sustained high intravascular concentration and low $k$ values (Table 1). It is, however, unclear whether a correlation should exist between $k$ and MVD values.

The superparamagnetic properties of USPIO may have caused an overestimation of the PV (Table 1). Intravascular USPIO particles produce a bulk magnetization that excites water protons beyond the vascular compartment, while gadopentetate dimeglumine produces a more local magnetization effect $(22,23)$. This may explain why USPIO f values were several times higher than gadopentetate dimeglumine $f^{p v}$ values (Table 1).

The high mobility and fast spread through the interstitial matrix of low molecular weight agents (35) may have negatively influenced the $f^{p V}$ measurements for gadopentetate dimeglumine, and this may explain their poor correlation with MVD (Table 2). In the tumor rim, USPIO $f^{\text {PV }}$ values also correlated poorly with MVD (Table 2). This may be explained by high angiogenic activity in the tumor rim with degradation of the intercellular matrix (2), which allows free movement of both USPIO and gadopentetate dimeglumine. We are aware that the use of $f^{p V}$ measurements has its limitations in this model. The $f^{p v}$ parameter is modeled as if the tissue has the same concentration-over-time course shape as the arterial input function. This may deviate from the true blood volume (11). Nevertheless, in our study, the $f^{P V}$ values measured with both agents revealed a higher plasma volume in the tumor rim than in the tumor core. The latter finding is in agreement with the MVD trend (Table 1).

Results and conclusions from our data are limited in several ways. Results from this human colon carcinoma model are preliminary, and their applicability remains to be proved in humans. Furthermore, MVD may not be the ideal surrogate marker of angiogenesis. Therefore, correlations between $K^{P S}$ and MVD observed for either agent cannot be perfect because co-registration between MR imaging sections and pathology sections is limited. Basically, MVD represents a morphologic marker, whereas dynamic contrast-enhanced MR imaging provides functional markers. Also, MVD assessments are inherently prone to sampling errors and are reported to be observer dependent, so they are at most semiquantitative. For instance, at low MVD values, the counting of stained vessels within a microscopic field is relatively easy and reliable. At higher MVD values, however, it is much more difficult to discern and count separate vessels because they may cluster. This inaccuracy might explain the larger scattering of data at high MVD values depicted in Figure 2. Furthermore, dysfunctional microvessels might affect MVD measurements because of difficulties in distinguishing between functional vessels and dysfunctional nonperfused vessels or endothelial cell clusters on histologic sections. Conversely, dynamic contrast-enhanced MR imaging parameters are affected only by functional vessels.

In spite of its shortcomings, MVD is generally considered the best standard for validating functional imaging techniques such as dynamic contrast-enhanced MR imaging, as long as these techniques are still being developed and need further improvement. Another limitation in our study may have been the two-compartment model we used, which has limited value in accounting for the complex and heterogeneous situation in a tumor 
environment; extended models with multiple compartments (36) may prove to be more accurate.

In addition, the relationship between the MR signal intensity and the concentration of contrast agent in vivo may deviate from the relationship determined in vitro due to compartmentalization effects, a limitation to the correct quantification of MR imaging microcirculation parameters that might be overcome by further improving the dynamic contrast-enhanced MR imaging technique. One way to do so is by speeding up the acquisition to obtain more sample points for the steep and error-prone arterial input function needed in the two-compartment model. High dynamic acquisition speeds are beneficial, particularly for low molecular weight contrast agents, to avoid enhancement of the extracellular compartment during the first pass of the bolus due to leakage from the circulation. But even for the USPIO used in the present study, some degree of leakage might be expected. Any degree of leakage of the USPIO used would mean that the USPIO may not be the ideal large-molecular reference contrast agent because it is not perfectly intravascular. At the dose used in this study the USPIO contrast agent yielded strong $\mathrm{T}^{*}$ effects, particularly during the bolus passage, that affected $\mathrm{T} 1$-weighted

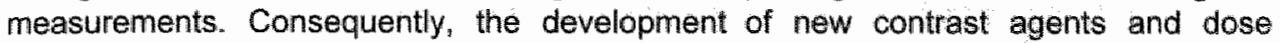
optimization are other potential methods to further improve dynamic contrast-entanced MR imaging of angiogenesis.

\section{Practical application}

Our findings suggest that the kinetic physiologic properties of gadopentetate dimeglumine are as good as those of USPIO for calculating a microvessel permeability parameter (ie, $K^{p S}$ ). Extended characterization of the tumor microenvironment, such as determination of the local blood volume fraction, might benefit more from new largemolecular contrast agents or optimized use of existing agents. Results of further work in which human subject protocols are used may show whether gadopentetate dimeglumine is indeed successful for diagnosing angiogenesis and monitoring the effects of antiangiogenic treatment in patients with colon cancer. 


\section{Appendix}

\section{Tracer kinetic madel}

The kinetic analysis was performed very similar to the method described by Daldrup ef al. (5) using a two-compartment model. Within the tumor these two compartments represent the blood plasma and interstitial (extravascular extracellular) space, and are assumed to be in equilibrium.

The dynamic exchange of the contrast agent between the two compartments amounts to the dynamic variation of the concentration of the contrast agent in the interstitial water of the tumor tissue $C_{1}(t)$ (mmol $\mathrm{cm}^{-3}$ of tissue), and is given by the differential equation

$$
d C_{1}(t) / d t=k^{P S} C_{p}(t)-k C_{t}(t)
$$

where $C_{p}(t)$ is the concentration of the contrast agent in the plasma space of the tumor tissue (mmoliml), $K^{P S}$ is the endothelial transfer coefficient $\left(\mathrm{ml} \mathrm{min} \mathrm{m}^{-1} \mathrm{~cm}^{-3}\right.$ of tissue)) and $k$ is the rate constant from interstitial water back to the plasma space $\left(\mathrm{min}^{-1}\right)$.

The concentration of the contrast agent in the tumor tissue at any time $t, C_{T}(t)\left(\mathrm{mmol}^{\mathrm{cm}}\right.$ ${ }^{3}$ of tissue) is composed of the contribution of both the interstitial and plasma content, and is given by

$$
C_{T}(t)=K^{P S} \int C_{P}\left(t^{\prime}-t_{0}\right) \exp \left(-k\left(t-t^{\prime}\right)\right) d t^{s}+f^{P W} C_{P}\left(t-t_{0}\right)
$$

where the $\mathrm{f}^{\mathrm{PV}}$ is the fractional plasma volume of the tumor tissue $\left(\mathrm{ml} \mathrm{cm} \mathrm{cm}^{-3}\right.$ of tissue) and the integral runs from to to $t$. The parameter $t_{0}$ represents the mean delay of the transit of the tracer from the supplying blood vessel to the tumor tissue.

The concentration of the contrast agent in the plasma at any time was related to the signal intensity ( $S \mid$ ) of the gradient echo images by the Ernst formula

$S \|=M_{0} \sin \alpha\left(1-E_{1}\right) /\left(1-E_{4} \cdot \cos \alpha\right) \exp \left(-T E / T_{2}^{*}\right)$

in which $M_{0}$ is the equilibrium magnetization depending on the proton density, $\alpha$ is the flip angle and $E_{1}=\exp \left(-T R / T_{1}\right)$ with $T R$ the repetition time and $T_{1}$ the longitudinal relaxation time. TE is the echo time and $T_{2}{ }^{*}$ is the transverse relaxation time. The shortening of the $T$, relaxation time due to the contrast agent is described by

$R_{1}=1 / T_{1}=1 / T_{10}+r_{1} C$

where $R_{1}$ is the $T_{1}$ relaxation rate, $T_{10}$ is the $T_{1}$ value before contrast injection (when $C=$ 0) and $r_{1}$ is the relaxivity of the contrast agent at $37^{\circ} \mathrm{C}$ and $1.5 \mathrm{~T}$. The values of $M_{0}^{*}=M_{0}$ $\exp \left(-\mathrm{TE} / \mathrm{T}_{2}{ }^{*}\right)$ and $\mathrm{T}_{40}$ were determined by fitting the Ernst formula to the signal intensities from the images acquired before contrast injection with different flip angles ( $\alpha$ $=2,5,10,15,25$, and $\left.35^{\circ}\right)$. Changes in the plasma $T_{1}$ relaxation rate $R_{4 p}$ are related to the measured changes in the blood $T_{1}$ relaxation rate $R_{1 B}$ by

$R_{1 p}=R_{1 B} /(1-H C t)$

where Hot is the hematocrit value. For the Gd-DTPA contrast agent only increases in signal intensity were noted in both the tumor tissue and in the aorta. Accordingly, the plasma concentration was calculated for the Gd-DTPA contrast agent as if $T_{2}^{*}$ effects 
due to the concentration variation were negligible. For the USPIO signal decreases were observed in the aorta reffecting strong $\mathrm{T}_{2}{ }^{*}$ effects at high tracer concentration. To relate the signal intensity to the concentration of the USPIO contrast agent use was made of

$$
R_{2}^{*}=1 / T_{2}^{*}=1 / T_{20}^{*}+r_{2}^{*} \mathrm{C}
$$

where $R_{2}{ }^{*}$ is the $T_{2}{ }^{*}$ relaxation, $1 / T_{20}{ }^{*}$ is the $T_{2}{ }^{*}$ value before contrast injection (thus $C=$ 0 ) and $r_{2}{ }^{*}$ is the relaxivity of the contrast agent at $37^{\circ} \mathrm{C}$ and $1.5 \mathrm{~T}$. It is stressed that this linear relation is an approximation which is applied for the vascular input function only. Due to the compartmentalization effect the resulting $\mathbb{R}_{2}{ }^{*}$ is expected to be unpredictable but larger in the tumor tissue. Voxels exhibiting no signal increase in the tumor tissue were omitted from the kinetic analysis.

The time course of the intravascular contrast concentration was used to determine an effective vascular input function (VIF). The VIF was calculated by fitting a decay curve to the blood contrast concentration time course data $\mathrm{C}_{B}(t)$ from the aorta. This decay curve consisted for the Gd-DTPA of two exponentials (i.e. 4 parameters) and for USPIO of one exponential (i.e. 2 parameters). Subsequently, the exponential model for the VIF was substituted in equation (2) yielding a multiple exponential expression. From the resulting expression the parameters $K^{P S}, k_{\mathrm{s}} \mathrm{f}^{\mathrm{PV}}$, and $\mathrm{t}_{0}$ were determined by fitting to the $\mathrm{C}_{\mathrm{T}}$ time course using the numerical Levenberg-Marquardt optimization algorithm. Pixels with a fit-error greater than $50 \%$ were rejected and omitted from the kinetic analyses. 


\section{References}

1. Folkman I. Anglogenesis research" from laboratory to clinic, Forum (Genova) 1999; 9: 59-62.

2. Griffioen, A. W. Molema, G. Angiogenesis: potentials for pharmacologic intervention in the treatment of cancer, cardiovascular diseases, and chronic inflammation, Phamacol Rev 2000; 52: $237-268$

3. Padhani, A. R. Dynamic contrast-enihanced MRi in clinical oncology: Current status and future

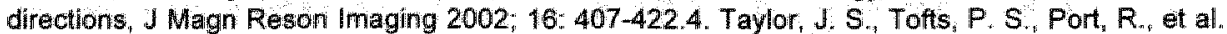
MR imaging of tumor microcirculation: promise for the new millennium, J Magn Reson Imaging 1999; 10: $903 \times 907$.

4. Dalldrup, H., Shames, D. M., Wendland, M., et al. Correlation of dynamic contrast-enhanced MR imaging with histologic fumor grade: comparison of macromolecular and small-molecular contrast media, AJR Am J Roentgenol 1998; 171:941-9.

5. Brasch, R. Turetschek, K. MRil characterization of tumors and grading angiogenesis using maciomolecular contrast media: status report. Eur J Radiol 2000; 34: 148-155.

6. Knopp, M. V., Weiss, E. Sinn, H. P., et all. Pathophysiollogic basis of contrast enhancement in breast tumors, J Magn Reson Imaging 1999; 10: 260-266.

7. Turetschek, K., Huber, S. Floyd, $E$., et al. MR imaging characterization of microvessels in experimental breast tumors by using a particulate contrast agent with histopathologic correlation, Readiology 2001; 218: 562-569.

8. Runge, V. M. Safety of approved MR contrast media for intravenous injection, J Magn Reson Imaging 2000; 12: 205-213.

9. Toth, E. Helm, L. Merbach, A. E. Relaxivity of gadolinium (III) complexes: theory and mechanism. In: Toth, E., ed. The Chemistry of Contrast Aglents in Medical Magnetic Resonance Imaging. 1 edn, West Sussex, UK, John Willey \& Sons, Ltd, 2001, pp. 45-119.

10. Henderson, E., Sykes, J., Drost, D., Weinmann, H. J., Rutt, B. K., Lee, T. Y. Simultaneous MRI measurement of blood flow, blood volume, and capillary permeability in mammary tumors using two different contrast agents, J Magn Reson Imaging 2000; 12: 991-1003.

11. Saini, S., Sharma, R., Baron, R. L., et al. Multicentre dose-ranging study on the efficacy of USPIO ferumoxtran-10 for liver MR imaging, Clin Radiol 2000; 55: 690-695.

12. Bjornerud, A., Johansson, L. O., Briley-Saebo, K. Ahlstrom, H. K. Assessment of $T 1$ and T2* effects in wivo and ex vivo using iron oxide nanoparticles in steady state-mdependence on blood volume and water exchange, Magn Reson Med 2002; 47:461-471.

13. Karczmar, G. S., Fan, X. Al-Hallaq, H. A., et al. Uptake of a superparamagnetic contrast agent imaged by MR with high spectral and spatial resolution, Magn Resan Med 2000; 43: 633-639.

14. Dennie, J., Mandeville, J. B., Boxerman, J. L., Packard, S. D., Rosen, B. R., Weisskoff, R. M. NMR imaging of changes in vascular morphology due to tumor angiogenesis, Magn Reson Med 1998; $40: 793-799$.

15. van Dijke, C. F. Brasch, R. C., Roberts, T, P., al Mammary carcinoma model: correlation of macromolecular contrast- enhanced MR imaging characterizations of tumor microvasculature and histologic capillary density, Radiology 1996; 198: 813-818.

16. Turetschek, $K_{w}$, Roberts, T. P. Floyd, E. al al. Tumor microvascular characterization using ultrasmall superparamagnetic iron oxide particles (USPIO) in an experimental breast cancer model J Magn Reson Imaging 2001; 13: 882-888.

17. Weinmann, H. J., Brasch, R. C., Press, W. R., Wesbey, G. E. Characteristics of gadoliniumDTPA complex: potential NMR contrast agent, AJR Am J Roentgenol 1984; 142: 619-624.

18. Brix, G, Semmler, W., Port, R., Schad, L. R., Layer, G., Lorenz, W. J. Pharmacokinetic parameters in CNS Gd-DTPA enhanced MR imaging. J Comput Assist Tomogr 1991; 15: 621628.

19. Niendorf, H. P. Haustein, J. Cornelius ${ } I_{x}$, Alhassan, A., Clauss, W. Safety of gadolinium-DTPA: extended clinical experience, Magn Reson Med 1991: 22: 222-228; discussion 229-232.

20. Leiner, T., Ho, $K_{\text {, Ho, }}$ V., Bongartz, G., Mali, W., Van Engelshoven, J. M. Multicenter trial of steady-state MR angiography of the peripheral arteries with NC100150 (Clariscan) for the evaluation of peripheral arterial disease. Paper presented at the International Society for Magnetic Resonance in Medicine, Proc. Intl. Soc. Mag. Reson. Mled 9: 42, Glasgow, Scotland, UK, 200\%, pp. 42 . 
21. Taylor, A. M., Panting, J.R., Keegan, J.y al. Safety and preliminary findings with the intravascular contrast agent NC100150 injection for MR coronary angiography. J Magn Reson Imaging 1999; 9: 220-227.

22. Muller, R. N., Roch, A., Colet, J. M., Ouakssim, A, Gollis, P. Paiticulate Magnetic Contrast Agents. In: Toth $E_{.,}$ed. The Chemistry of Contrast Agents in Medical: Magnetic Resonance Imaging. 1 edn, West Sussex, UK, John Wiley \& Sons, Ltd, 2001, pp, 417-435.

23. Haacke, M., Brown, R. W. Thompson, M. R., Venkatesan, R. T1 estimation from SSI measurements at multiple flip angles. In: Venkatesan, R., ed. Magnetic resonance imaging: physical principles and sequence design. 1 edn, New York, NY, John Wiley \& Sons, Inc., 1999, pp. 654-661.

24. Tofts, $P . S$., Kermode, A. G. Measurement of the blood-brain barrier permeability and leakage space using dynamic MR imaging. 1. Fundamental concepts Magn Reson Med 1991: 17: 357 . 367.

25. Tofts, $P$. S., Johnson, $G$., Cha, S. A simple model to characterize blood-brain barrier leakage from T2* -weighted bolus tracking MRI data. Paper presented at the International Society for Magnetic Resonance in Medicine, Proc Intl. Soc. Mag. Reson. Med 9: 2242, Glasgow, Scotland, UK, 2001 , pp. 2242.

26. Zar, $\mathbb{U}$. H. Comparing Two Correlation Coefficients. lin: Zar, J. H, ed. Biostatistical Analysis, 3 edn, Upper Saddle River, $N J_{\text {o }}$ Prentice-Hall International " Inc., 1996, pp. 375-382.

27. Hobbs, S. K., Monsky, W. L., Yuan, F., et all. Regulation of transport pathways in tumor vessels: role of tumor type and microenviromment, Proc Natl Acad Sci U S A 1998; $95: 4607-12$.

28. Kroft, $L$. J., de Roos, A. Blood pool contrast agents for cardiovascular MR imaging, J Magn Reson Imaging 1999; 10: 395-403.

29. Buckley, D. L. Uncentainty in the analysis of tracer kinetics using dynamic contrast-enhanced T(1)-weighted MRI, Magn Reson Med 2002; 47: 601-606.

30. Port, R. E., Knopp, M. V., Brix, G. Dynamic contrast-enhanced MRI using Gd-DTPA: interindividual variability of the arterial input function and consequences for the assessment of kinetics in tumors, Magn Reson Med 2001; 45: 1030-1038.

31. LI, K. L., Zhu, X. P., Waterton, J., Jackson, A. Improved 3D quantitative mapping of blaod volume and endothelial permeability in brain tumors, J Magn Reson Imaging 2000; 12: 347-357,

32. Zhu, X. P., Li, K. L., Kamaly-AsI, $L_{\text {. }}$. . et al. Quantification of endothelial permeability, leakage space, and blood volume in brain tumors using combined T1 and T2* contrast-enhanced dynamic MR imaging, J Magn Reson Imaging 2000; 11: 575-585.

33. Haacke, M., Brown, R. W., Thompson, M. R., Venkatesan, R. Magnetic properties of tissues: theory and measurement. In: Venkatesan, R., ed. Magnetic resonance imaging: physical principles and sequence design. 1 edn, New York, NY. John Wiley \& Sons, llnc., 1999, pp. 741. 765.

34. Jain, R. K. Delivery of molecular medicine to sollid tumors: lessons from in vivo imaging of gene expression and function, $J$ Control Release $2001 ; 74: 7-25$.

35. Henderson, E., Sykes, J. Drost, D., Weinmann, H. J., Rutt, B. K., Lee, T. Y. Simultaneous MRI measurement of blood flow, blood volume, and capillary permeability in mammary tumons using two different contrast agents, J Magn Reson Imaging 2000; 12: 991-1003.

36. Port, R. E., Knopp, M. V. Hoffmann, U., Milker-Zabel, S. Brix, G. Multicompartment analysis of gadolinium chelate kinetics: blood-tissue exchange in mammary fumors as monitored by dynamic MR imaging, J Magn Reson Imaging 1999; 10: 233-241. 


\title{
Chapter 3
}

\section{Relationship Between DCE-MRI Kinetic Parameters and Molecular Weight of Dendritic Contrast Agents in Tumor Angiogenesis in Mice}

\author{
Radiology, 2005 February: 235(1)65-72
}

Quido G. de Lussanet, Sander Langereis, Regina G. H. Beets-Tan, Marcel H. P. van Genderen, Arjan W. Griffioen, Jos M. A. van Engellshoven, Walter $\mathrm{H}$. Backes.

\section{ABSTRACT}

\section{PURPOSE}

To evaluate the relationship between dynamic contrast agent-enhanced magnetic resonance (MR) imaging-derived kinetic parameters and contrast agents of equal chemical composition and configuration but with different molecular weights in a tumor angiogenesis model.

\section{MATERIALS AND METHODS}

This study was approved by the ethical review committee. Maintenance and care of animals was in compliance with guidelines set by the institutional animal care committee. Dynamic contrast-enhanced MR imaging was performed with dendritic contrast agents in 16 mice with tumor xenografts; mice were placed in groups of four for each molecular weight of the contrast agent. The magnitude and spatial distribution of kinetic parameters (transfer coefficient $\left[K^{P S}\right]$ and plasma fraction $\left[f^{P V}\right]$ ) were compared with molecular weight of the contrast agent by determining the Spearman correlation coefficient $(r)$ and the quantitative relationship between the endothelial $K^{p S}$ and molecular weight.

\section{RESULTS}

Inverse relationships between molecular weight of contrast agent and $K^{p s}$ and $f^{p V}$ of tumor $\operatorname{rim}(r=-0.8, P<.001$ and $r=-0.5, P=.04$, respectively) and core $(r=-0.7, P=$ .004 and $r=-0.6, P=.01$, respectively) were observed. The quantitative relationship between $K^{P S}$ and molecular weight (MW) was $K^{P S}=0.4 / M^{0} W^{0.44}$. A decreasing stepwise pattern in $\mathrm{f}^{\mathrm{PV}}$ was noted between contrast agents with low $(0.7-$ and $3.0-\mathrm{kDa})$ molecular weight and those with high $(12-$ and $51-\mathrm{kDa})$ molecular weight.

\section{CONCLUSION}

Macromolecular permeability is best measured with high-molecular-weight contrast agents; endothelial $\kappa^{\text {PS }}$ values measured with low-molecular-weight contrast agents incorporate tissue perfusion and permeability and demonstrate heterogeneous microcirculatory flow. 


\section{Introduction}

Recognition that angiogenesis plays an essential role in tumor development and metastasis formation has led to new developments in the diagnosis and treatment of cancer (1-3). A promising development is the in wivo characterization of tumor angiogenesis and monitoring effects of antiangiogenesis treatment by means of dynamic contrast agent-enhanced magnetic resonance (MR) imaging (4). Dynamic contrastenhanced MR imaging data may be subjected to pharmacokinetic analysis $(5,6)$ to derive quantitative parameters that reflect tumor microcirculation characteristics and angiogenic activity. Angiogenic properties are vasodilatation, increased microvessel permeability, and vessel sprouting and remodeling, which may lead to increases both in heterogeneity and in tumor blood volume and flow level. These properties are reflected by dynamic contrast-enhanced MR imaging data $(1,7,8)$, MR imaging-derived parameters that reflect angiogenic activity include endothelial transfer coefficient $\left(K^{P S}\right)$ and plasma fraction ( $f^{\mathrm{PV}}$ ). $\mathrm{K}^{\mathrm{PS}}$ was shown to correlate with histologic microvessel density and tumor grade, and it has been used to monitor the effects of antiangiogenesis treatments in different tumor models $(4,9-13)$.

One area of discussion in the field of dynamic contrast-enhanced MR imaging of tumor angiogenesis is the choice of contrast agent (4), particularly the molecular weight or particle size of the contrast agent. Results of previous experimental comparison studies performed with different contrast agents in tumor angiogenesis models have suggested that molecular weight of the contrast agent affects the measured $K^{P S}$ and $f^{p v}$ values $(12,14)$. For example, $K^{\mathbb{P S}}$, which represents the rate of transfer of contrast agent from the blood to the interstitial space, is highly dependent on the permeability and surface area of the endothelium. As contrast agents with a low molecular weight have a large first-pass extraction, $K^{P S}$ values are dominated by flow in tumors. Alternatively, contrast agents with a high molecular weight leak from the blood more slowly, and transfer into the interstitial matrix is limited in relation to tumor blood flow. In this situation, $K^{P S}$ values will approximate the permeability surface area product. The $K^{P S}$ of contrast agents with an intermediate molecular weight is influenced both by permeability surface area product and by flow (i.e.. flow contamination).

In tumor angiogenesis, microvessel permeability and surface area may be greatly increased, but the microcirculatory flow may also be increased and highly heterogeneous $(2,7,8)$. Thus, increases in flow levels and heterogeneity may be best assessed by using low-molecular-weight contrast agents. The $\mathrm{f}^{\mathrm{PV}}$ parameter might be best measured by using a high-molecular-weight MR contrast agent because of relatively long intravascular life times, and it might be overestimated when using lowmolecular-weight contrast agents that rapidly diffuse from the vascular compartment to the interstitial space. These discussions have resulted from experimental work by using a variety of contrast agents $(12,14)$. The contrast agents that have been evaluated to date (i.e. low-molecular-weight gadolinium complexes, gadolinium complexes conjugated to linear polymers or albumin, and iron oxide particles) (4,9-14), however, may have been too different in molecular composition and paramagnetic properties to allow fair comparisons between different agents.

Gadolinium-based dendrimers are macromolecular MR contrast agents with well-defined molecular weights; most important, they are of equal chemical composition $(15,16)$. The properties of gadolinium-based dendrimers suggest that these dendritic contrast agents might be ideal for comparing molecular weights for MR imaging of tumor angiogenesis. 
Thus, the purpose of our study was to evaluate the relationship between dynamic contrast-enhanced MR imaging-derived kinetic parameters and contrast agents of equal chemical composition and configuration but with different molecular weights in a tumor angiogenesis model. We hypothesized that $K^{P S}$ and $f^{p V}$ would increase as the molecular weight of the MR contrast agent decreased.

\section{Materials and Methods}

\section{Synthesis of dendritic MR contrast agents}

Different generations of poly(propylene imine) dendrimers (Figure 1) (16) were modified with the gadolinium diethylenetriaminepentaacetic acid moiety and then underwent complexation with gadolinium chloride to create well-defined gadolinium diethylenetriaminepentaacetic acid-based dendritic contrast agents with different molecular weights. The molecular and paramagnetic properties of the agents are presented in Table 1 (16). The polydispersity of the poly(propylene imine) dendrimers has been measured with electrospray mass spectrometry, and it was found to be less than 1.003 for all dendrimers (17). These dendritic contrast agents represent a class of well-defined highly branched macromolecular architectures, with a precise number of gadolinium diethylenetriaminepentaacetic acid complexes located at the periphery (18). The multivalent nature of dendrimers provides a way of introducing different functionalities, such as tracer units, targeting entities, and/or ather therapeutic molecular constructs, to the same molecule.
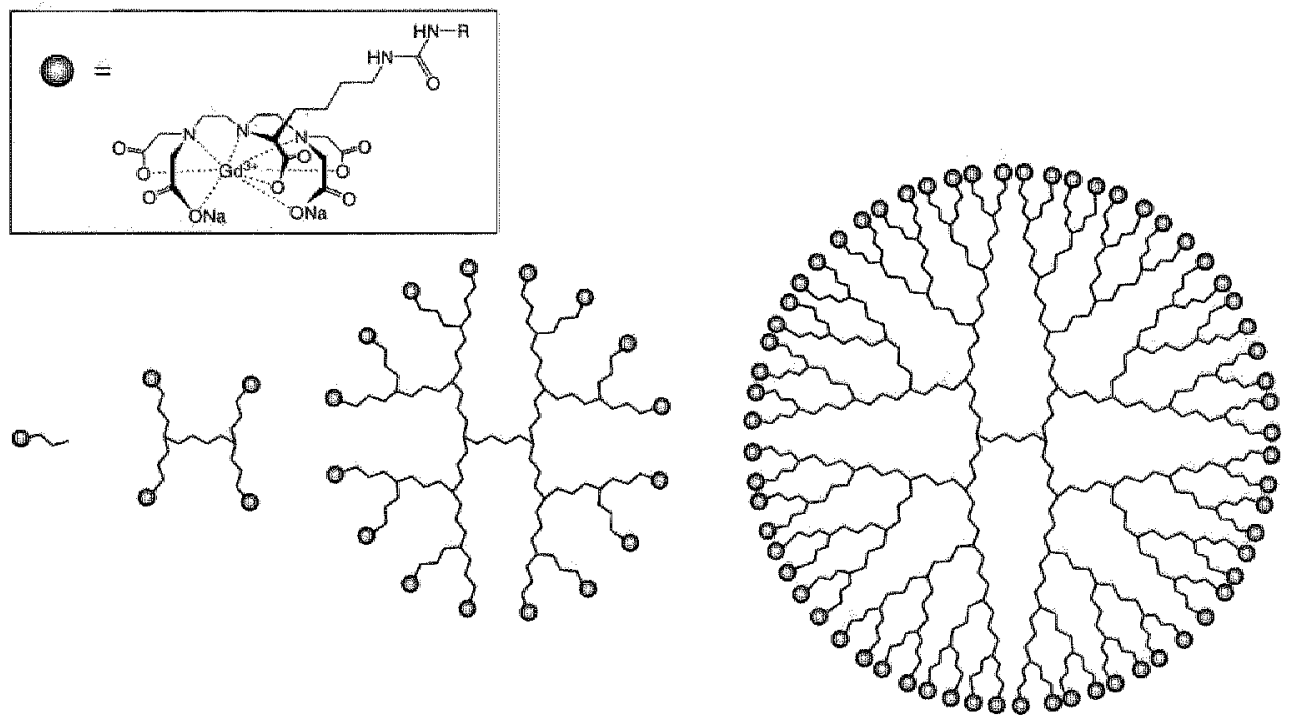

Figure 1. Schematic presentation of the different generations of poly(propylene imine) dendrimers, with the gadolinium chelating diethylenetriaminepentaacetic acid (DTPA) molety complexated with gadoliniumchloride (insert). The number of gadolinium complexes, from left to right, are 1, 4, 16 and 64 , respectively. 


\section{Animal model}

This study was approved by the ethical review committee at our institution (Maastricht University Hospital, Maastricht, the Netherlands). The maintenance and care of the experimental animals were in compliance with the guidelines set by the institutional animal care committee, which is accredited by the Netherlands National Department of Health. Sixteen male nude mice (Swiss nu/nu; Charles River, Maastricht, the Netherlands) (age, 9 weeks) received an injection of $10^{6}$ cells of LS 174T human collon carcinoma cells subcutaneously in the left flank. Sixteen days after tumor cell injection, the mice underwent MR imaging after being anesthetized with subcutaneous injection of $100 \mathrm{mg}$ per kilogram of body weight of ketamine (Nimatek; Eurovet, Bladel, the Netherlands) and $10 \mathrm{mg} / \mathrm{kg}$ of xylazine hydrochloride (Sedamun; Eurovet). The mice were randomly assigned to receive one of the four dendritic MR contrast agents (gadolinium dose; $0.03 \mathrm{mmol} / \mathrm{kg}$ ). The contrast agent was injected slowly into the tail vein and flushed with $15 \mu \mathrm{L}$ of normal saline ( $\mathrm{NaCl} 0.9 \%$ injection fluid; Braun, Melsungen ${ }_{i}$ Germany) during the fifth dynamic volume acquisition: this procedure required approximately 40 seconds. Warm water bags were placed near the mouse to keep the temperature in the MR imaging unit bore near $28^{\circ} \mathrm{C}$. After imaging and while the mice were still anesthetized, they were sacrificed by means of cervical dislocation.

\section{MR imaging}

MR imaging was performed as described previously (10), with a small surface coll (diameter $5 \mathrm{~cm}$ ) and a 1.5-T system (Philips Medical Systems, Best, the Netherlanids). The imaging protocol included a T2-weighted anatomic acquisition (multisection fast spin echo, echo train length of 28 , repetition time msec/echo time msec of $3300 / 200$, and flip angle of $90^{\circ}$ ) for locating and delineating the tumor, a pre-contrast $\mathrm{T} 1$-weighted measurement (three-dimensional fast field echo, 50/7, and flip angles of $2^{\circ}, 5^{\circ}, 10^{\circ}, 15^{\circ}$, $25^{\circ}$, and $35^{\circ}$ ), and a T1-weighted dynamic contrast enhanced series (three-dimensional fast field echo, $50 / 7$, and flip angle of $35^{\circ}$ ) with 55 dynamic volume acquisitions of 39 seconds each. The T1- and T2-weighted acquisitions had the same positioning with 16 transverse sections. Sections were $2.0 \mathrm{~mm}$ thick for T2-weighted acquisitions and 4.0 mm thick for T1-weighted acquisitions, for which adjacent sampled sections were displaced $2.0 \mathrm{~mm}$ and subsequently interpolated to $2.0-\mathrm{mm}$-thick sections during reconstruction. Matrix dimensions were $128 \times 128$, with a field of view of $64 \times 64 \mathrm{~mm}$; reconstructed voxel sizes were $0.5 \times 0.5 \times 2.0 \mathrm{~mm}$.

\section{Analysis of MR imaging data}

MR imaging data were analyzed by using a general kinetic two-compartment bidirectional exchange model as described previously (19) in the Matlab programming

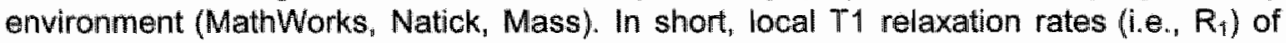
the pre-contrast time-averaged images and post-contrast image signal intensity time courses were used to determine the plasma concentration of the contrast agent in the aorta, which represents the arterial input function for each mouse individually and the tissue concentration in the tumor. Voxel-wise signal intensity time courses were converted to $T 1$ relaxation rates $\left(R_{1}[t]\right)$. Application of the general kinetic twocompartment bi-directional exchange model $(10,19)$ yielded the $K^{\text {Ps }}$ and reflux rate $(k)$ values, both of which are measured in milliliters per minute per $100 \mathrm{~cm}^{3}$ of tissue, and the $f^{p v}$ value, which is expressed as milliliters per $100 \mathrm{~cm}^{3}$ of tissue. The reflux rate describes the transfer from the interstitial space back to the plasma space. Kinetic analysis was performed for each tumor voxel in the central section through the tumor. 
Values for individual voxels were (a) collected for histogram analysis of $K^{p s}$; (b) averaged to obtain whole tumor values of $K^{\rho S}, k$, and $f^{p v}$ values for each tumor; and (c) used to create a color-coded map of $K^{P S}$ values for each tumor. To allow for inferences on differences between tumor rim and tumor core, regions of interest were drawn in consensus to delineate the peripheral tumor margin (i.e., rim) (thickness, about $2 \mathrm{~mm}$ ) from the remaining central (i.e., core) portion, respectively, by using the T2-weighted anatomic image.

To quantify the relationship between the mean whole tumor $K^{P S}$ value and molecular weight $(\mathrm{MW})$ of the contrast agent, an author (W.H.B.) determined the parameters $a$, which is the $K^{P S}$ for molecular weight equal to $1 \mathrm{kDa}$, and power $\gamma$ in the mathematic expression $K^{P S}=a /(M W)^{\psi}$ by using a minimum of least-squares linearized fitting procedure. Linearization of this equation was performed with log transformation of both sides: $\log K^{P S}=(\log a)-y(\log M W)$. After linearization, linear regression was applied $(x$ $=\log M W$ and $\left.y=\log K^{P S}\right)$ to yield the slope $(y)$.

Blood circulation times and renal clearance rates were approximated for the dendritic contrast agents by means of visual comparison of normalized signal enhancement time courses for regions of interest drawn by means of consensus in the aorta, renall parenchyma, renal pelvis, and bladder.

\section{Statistical analysis}

The number of animals included was estimated by performing a power analysis (20) ( $95 \%$ confidence interval and $80 \%$ power) and considering a predetermined possible dropout rate of less than $10 \%$; this amounted to four mice per contrast agent group, for a total of 16 animals. All pixels in the central MR section in each tumor (i.e. "tumor volume) were averaged for each contrast agent group and compared by using the two-tailed Student $t$ test. Molecular weights of the contrast agents and the tumor volumes were compared with (a) average $K^{P S}, k$, and $f^{P V}$ values for the whole tumor volume, tumor rim, and tumor core; (b) the rim-core ratio, defined as the quatient of tumor rim divided by tumor core values; and (c) the spatial heterogeneity, defined as the second moment (i.e., the standard deviation) of the relative distribution of the voxel $K^{P S}$ values in a tumor, by using the one-tailed Spearman rank correlation coefficient, which allows for non-normal distribution of variables. Histograms, normalized for the tumor extent, were created with voxel $K^{P S}, k$, and $f^{P V}$ walues for each contrast agent to allow us to visually assess the distribution of the variables. Average $K^{\mathrm{PS}}, k$, and $\mathrm{f}^{\mathrm{PV} V}$ values for the whole tumor, tumor rim, and tumor core were compared between the different contrast agent groups. Averaged whole tumor values for the 0.7-and 3.0-kDa groups were compared with those for the 12 - and $51-\mathrm{kDa}$ groups by using the one-tailed Student $t$ test. Statistical analyses were performed using commercial software (SPSS 11.0.1; SPSS, Chicago, III), and a $P$ value of less than .05 was considered to indicate a statistically significant difference.

\section{Results}

Fifteen of the 16 mice were successfully imaged; 12 mice (four in each group) were in the $0.7-, 3.0$, and 51-kDa,groups, and three mice were in the 12-kDa group. One mouse was not successfully imaged because of a technical error during dynamic MR imaging. Tumor volumes ranged from 46 to 777 voxels (average number of voxels \pm standard deviation, $305 \pm 208$ ) (Table 2). The average number of voxels per tumor did not differ significantly $(P>4)$ between the contrast agent groups (Table 2) and showed no 
relationship with MR parameters $(0.1<r<0.3 ; P>3)$. All contrast agents were cleared from circulation through the renal system. The $51 \mathrm{kDa}$ contrast agent was cleared through the renal system at a slower rate than the 0.7 - and 3.0-kDa contrast agents, and brief accumulation in the hepatic and splenetic system was observed for the $54-\mathrm{kDa}$ contrast agent.

$K^{p}$ values

Our results showed a strong inverse relationship $(r=-0.7, P=001)$ between the molecular weight of the dendritic contrast agent and the whole-tumor $K^{P S}$ values (figure 2a). The inverse relationship between $K^{\mathrm{SS}}$ and molecular weight was noted for both tumor rim $(r=-0.8, P<.001)$ and tumor core $(r=-0.7, P=.004) k^{P S}$ values. Lowmolecular-weight contrast agents had the highest $K^{\text {PS }}$ values, and vice versa (Figure 2a). Fitting the proposed power relationship between $k^{p S}$ and molecular weight yielded the following parameter values: $a=0.41 \pm 0.15$ and $y=0.44 \pm 0.14$

a

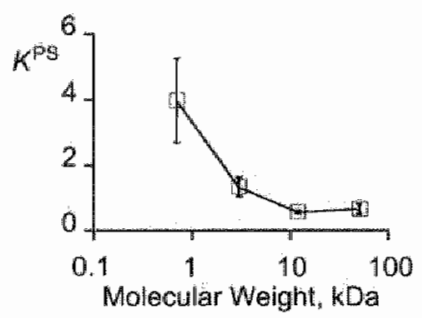

b

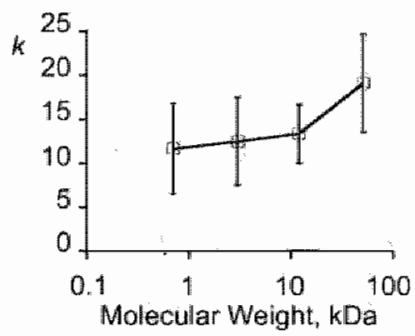

$\mathrm{C}$

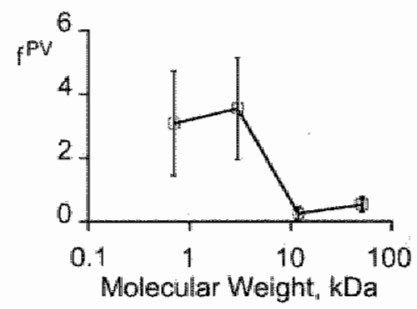

Figure 2. Graphs of average whole tumor values illustrate (a) a neglative relationship between $K^{p s}$ and molecular weight, (b) a possible positive relationship between $k$ and molecular weight and (c) a threshold effect with a marked decrease of $\mathrm{fV}^{\mathrm{V}}$ between 3 and $12 \mathrm{kDa}$ molecular weight af dendritic contrast agent. The error bars indicate standard errors of the mean and illustrate the inter-tumor variation in the different contrast agent groups. The error bars (standard errors of the mean) illustrate greater inter-tumor variation in $\mathrm{K}^{\mathrm{PS}}$ and $f^{\mathrm{PV}}$ for low molecular weight weight dendritic contrast agents than for large molecular weight agents, whereas the standard error in $k$ values was equivalent for the different molecular weight agents.

Histogram analysis (Figure 3 ) showed greater ranges in distribution and higher mean $K^{p S}$ values for contrast agents with lower molecular weight $(0.7$ or $3.0 \mathrm{kDa})$ than for those with higher molecular weight $(12$ or $51 \mathrm{kDa}$ ). The differences in ranges in distribution of $K^{P S}$ for the different contrast agents (Figure 3 ) showed a significant $(r=-0.8, P<.001$ ) inverse relationship with the molecular weight of the contrast agent. Pooled mean $K^{p s}$ values for low-molecular-weight $(0.7-$ and $3.0-\mathrm{kDa})$ contrast agents were about four times greater $(P=.02)$ than those for high-molecular-weight (12-and $51-\mathrm{kDa}$ ) contrast agents (Table 2). 
Table 1. Contrast Agent Characteristics

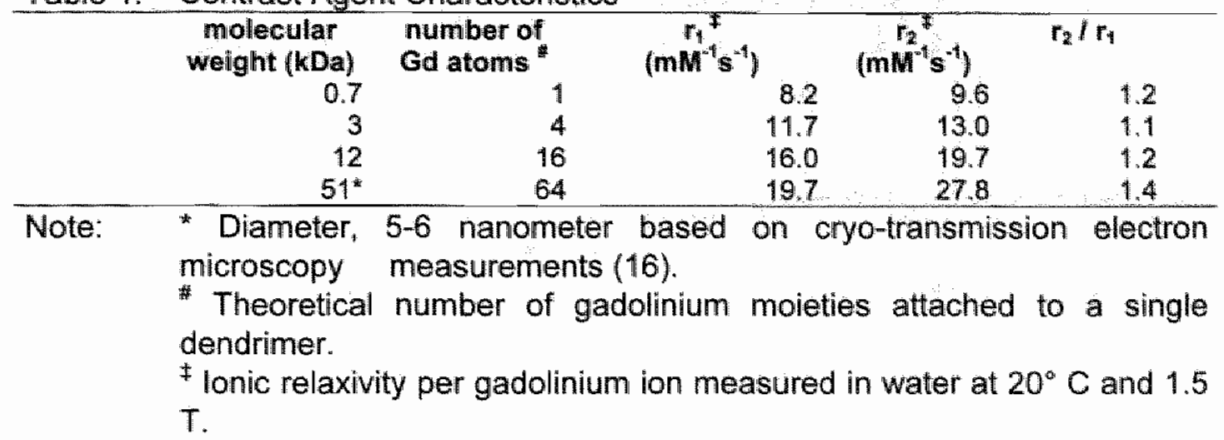

Table 2. Dynamic Contrast-enhanced MR Imaging Derived Parameters in Terms of Ascending Molecular Weight

\begin{tabular}{|c|c|c|c|c|c|c|c|c|c|c|}
\hline \multirow{2}{*}{$\underset{\text { (kDa) }}{\text { MW }}$} & \multirow{2}{*}{$\begin{array}{c}\text { tumor } \\
\text { volume } \\
*\end{array}$} & \multicolumn{3}{|c|}{$K^{P S H}$} & \multicolumn{3}{|c|}{$k^{*}$} & \multicolumn{3}{|c|}{$f^{P V / 8}$} \\
\hline & & tumor & rim & core & tumor & rim & core & tumor & $\mathrm{rim}$ & core \\
\hline $\begin{array}{l}0.7 \\
0.7 \\
0.7 \\
0.7\end{array}$ & $\begin{array}{r}325 \\
164 \\
67 \\
329 \\
267 \pm 177\end{array}$ & $\begin{array}{r}7.3 \\
2.6 \\
4.5 \\
1.5 \\
4.0 \pm 2.6\end{array}$ & $\begin{array}{r}8.9 \\
3.3 \\
4.8 \\
1.8 \\
4.7 \pm 3.1\end{array}$ & $\begin{array}{r}1.6 \\
1.0 \\
1.1 \\
1.1 \\
1.2 \pm 0.3\end{array}$ & $\begin{array}{r}26.3 \\
10.8 \\
4.4 \\
5.0 \\
12 \pm 10\end{array}$ & $\begin{array}{r}33.0 \\
15.2 \\
3.9 \\
5.1 \\
14 \pm 14\end{array}$ & $\begin{array}{l}4.8 \\
1.8 \\
2.7 \\
6.3 \\
4 \pm 2\end{array}$ & $\begin{array}{l}7.7 \\
3.4 \\
1.2 \\
0.3 \\
3 \pm 3\end{array}$ & $\begin{array}{l}5.2 \\
3.4 \\
1.2 \\
0.2 \\
3 \pm 2\end{array}$ & $\begin{array}{r}10.0 \\
2.0 \\
2.0 \\
0.2 \\
4 \pm 4\end{array}$ \\
\hline $\begin{array}{l}3 \\
3 \\
3 \\
3\end{array}$ & $\begin{array}{r}218 \\
246 \\
189 \\
777 \\
398 \pm 267\end{array}$ & $\begin{array}{r}0.5 \\
1.3 \\
2.0 \\
1.5 \\
1.3 \pm 1.6\end{array}$ & $\begin{array}{r}0.6 \\
1.3 \\
2.1 \\
2.6 \\
1.6 \pm 0.9\end{array}$ & $\begin{array}{r}0.4 \\
1.1 \\
0.8 \\
0.5 \\
0.7 \pm 0.3\end{array}$ & $\begin{array}{r}15.8 \\
25.0 \\
3.7 \\
5.4 \\
13 \pm 10\end{array}$ & $\begin{array}{r}21.4 \\
29.3 \\
3.5 \\
8.5 \\
16 \pm 12\end{array}$ & $\begin{array}{r}13.6 \\
23.3 \\
2.1 \\
1.9 \\
10 \pm 10\end{array}$ & $\begin{array}{l}0.5 \\
7.9 \\
2.4 \\
3.4 \\
4 \pm 3\end{array}$ & $\begin{array}{l}0.1 \\
7.1 \\
1.1 \\
4.8 \\
3 \pm 3\end{array}$ & $\begin{array}{r}0.4 \\
1.7 \\
0.2 \\
1.6 \\
1 \pm 1\end{array}$ \\
\hline $\begin{array}{l}12 \\
12 \\
12\end{array}$ & $\begin{array}{r}209 \\
271 \\
715 \\
358 \pm 281\end{array}$ & $\begin{array}{r}0.5 \\
0.6 \\
0.6 \\
0.6 \pm 0.1\end{array}$ & $\begin{array}{r}0.6 \\
0.6 \\
0.9 \\
0.7 \pm 0.2\end{array}$ & $\begin{array}{r}0.5 \\
0.4 \\
0.6 \\
0.5 \pm 0.1\end{array}$ & $\begin{array}{r}9.8 \\
10.2 \\
20.0 \\
13 \pm 6\end{array}$ & $\begin{array}{l}13.5 \\
10.4 \\
18.0 \\
14 \pm 4\end{array}$ & $\begin{array}{r}7.7 \\
9.1 \\
21.4 \\
13 \pm 8\end{array}$ & $\begin{array}{r}0.1 \\
0.1 \\
0.7 \\
0.3 \pm 0\end{array}$ & $\begin{array}{c}0.2 \\
0.1 \\
1.2 \\
1 \pm 1\end{array}$ & $\begin{array}{r}0.1 \\
0.1 \\
0.4 \\
0.1 \pm 0\end{array}$ \\
\hline $\begin{array}{l}51 \\
51 \\
51 \\
51\end{array}$ & $\begin{array}{r}479 \\
272 \\
46 \\
272 \\
261 \pm 128 \\
\end{array}$ & $\begin{array}{r}0.7 \\
0.9 \\
0.8 \\
0.3 \\
0.7 \pm 0.3 \\
\end{array}$ & $\begin{array}{r}0.8 \\
1.0 \\
1.3 \\
0.4 \\
0.9 \pm 0.4 \\
\end{array}$ & $\begin{array}{r}0.6 \\
0.9 \\
0.5 \\
0.2 \\
0.6 \pm 0.3 \\
\end{array}$ & $\begin{array}{r}10.5 \\
19.4 \\
11.9 \\
34.4 \\
19 \pm 111 \\
\end{array}$ & $\begin{array}{r}8.1 \\
22.2 \\
13.0 \\
47.8 \\
23 \pm 18 \\
\end{array}$ & $\begin{array}{l}13.2 \\
15.9 \\
11.8 \\
21.1 \\
16 \pm 4\end{array}$ & $\begin{array}{r}1.1 \\
0.3 \\
0.1 \\
0.6 \\
0.5 \pm 1 \\
\end{array}$ & $\begin{array}{l}2.5 \\
0.5 \\
0.1 \\
1.0 \\
1 \pm 1\end{array}$ & $\begin{array}{r}0.3 \\
0.4 \\
0.1 \\
0.2 \\
0.240 \\
\end{array}$ \\
\hline Note. & $\begin{array}{l}\text { Mean I } \\
\text { significant } \\
\text { \# Mean [ } \pm \\
.04 \text { ) and } 5 \\
K^{P S} \text { for } 3 \mathrm{k} \\
{ }^{\ddagger} \text { Mean [ } \pm \\
\text { groups. M } \\
{ }^{5} \text { Mean [ } \pm \\
\text { the differe }\end{array}$ & $\begin{array}{l}{[ \pm \text { SDI nu }} \\
\text { ly different } \\
\text { SD] wholl } \\
1 \mathrm{kDa}(P \\
\text { Da was sig } \\
\text { SD] } k \text { for } \\
\text { ean tumor } \\
\text { SD] ffv fo } \\
\text { nt groups. }\end{array}$ & $\begin{array}{l}\text { Imber of } \\
(P>4) \\
e \text { tumor } \\
=.02) \text { bu } \\
\text { gnificantly } \\
\text { the whole } \\
\text { core } k \text { for } \\
\text { of the who }\end{array}$ & $\begin{array}{l}\text { pixels in } \\
\text { etween th } \\
\text { for } 0.7 \\
\text { t not sign } \\
\text { higher th } \\
\text { tumor sh } \\
0.7 \mathrm{kDa} \\
\text { le turnor }\end{array}$ & $\begin{array}{l}\text { he centr } \\
\text { different } \\
\text { Da was } \\
\text { cantly hi } \\
\text { for } 12 \mathrm{k} \\
\text { wed no } \\
\text { s signifi } \\
\text { lowed } n\end{array}$ & $\begin{array}{l}\text { MR se } \\
\text { ontrasit } \\
\text { ignifican } \\
\text { ier than } \\
(P=.0 \\
\text { nificant } \\
\text { nily high } \\
\text { significa }\end{array}$ & $\begin{array}{l}\text { ction in } \\
\text { gent gro } \\
\text { ly highe } \\
\text { for } 3 \mathrm{kD} \\
\text { 4) and } \\
\text { differenc } \\
\text { er than } \\
\text { th }(P>\end{array}$ & $\begin{array}{l}\text { each } \\
\text { oups. } \\
\text { than } \mathrm{f} \\
51 \mathrm{kDa}( \\
\text { ce betwe } \\
\text { for } 51 \mathrm{~kL} \\
.05) \text { diffe }\end{array}$ & $\begin{array}{l}\text { Mear } \\
=.04 \text { ). } \\
\text { the d }\end{array}$ & $\begin{array}{l}\text { las not } \\
\text { lumor } \\
\text { tumor } \\
\text { lifferent } \\
\text { 003). } \\
\text { etween }\end{array}$ \\
\hline
\end{tabular}



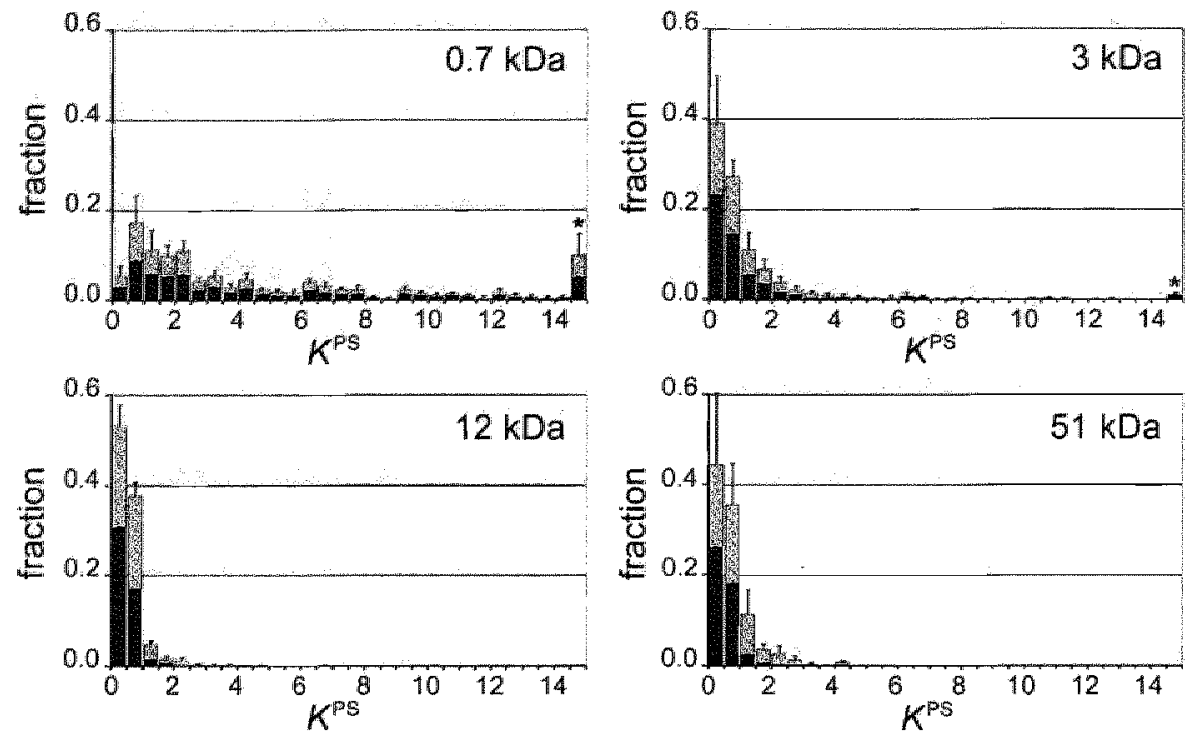

Figure 3. Normalized histograms illustrate that low molecular weight contrast agents result in much greater range in spatial distribution (ie, intra-tumor variation) of the measured $K^{p S}$ values in individual voxels compared with the higher molecular weight contrast agents. Each interval column represents the tumor values of which the black (lower) part represents tumor core values and the shaded (upper) part represents tumor rim values. The error bars represent the standard error of the mean, and illustrate the inter-tumor variation in the different contrast agent aroubs. * $K^{p s}$ values of 14.5 and hiaher were pooled.

Color-coded $K^{\mathrm{PS}}$ tumor maps show that the highest $K^{\mathrm{PS}}$ values were typically measured with the lowest-molecular-weight contrast agents and in the tumor rim (Figure 4). Ratios of tumor rim and tumor core $K^{P S}$ values (average ratio of $4.0 \pm 1.9,2.7 \pm 1.8,1.6 \pm 0.2$, and $1.7 \pm 0.6$ for the $0.7-, 3.0-, 12-$, and $51-\mathrm{kDa}$ groups, respectivelly) decreased significantly $(r=-0.6, P=.01)$ with increasing molecular weight.

\section{Reflux rate}

We were unable to identify relationships between molecular weight and whole-tumor ( $r=$ $0.3, P=1$ ) (Figure 2b) and tumor rim $k$ values $(r=0.3, P=.2)$, although a significant positive relationship was found for tumor core $k$ values $(r=0.6, P=.005$ ) (Table 2). Spatial distribution in $k$ values showed no relationship $(r=0.1, P=4)$ with molecular weight; whole-tumor $\mathrm{k}$ values for the 0.7 - and $3.0-\mathrm{kDa}$ contrast agents were not significantly different $(P=.2)$ from those for the 12 - and $51-\mathrm{kDa}$ agents. Ratios of tumor rim and tumor core $\mathrm{k}$ values (average rim-core $\mathrm{k}$ ratio of $4.5 \pm 3.9,2.2 \pm 1.6,1.3 \pm 0.5$, and $1.4 \pm 0.7$ for the $0.7-, 3.0-, 12-$, and $51-\mathrm{kDa}$ groups, respectively) did not show a significant inverse relationship $(r=-0.4, P=.1)$ with the molecular weights of the MR contrast agents. 


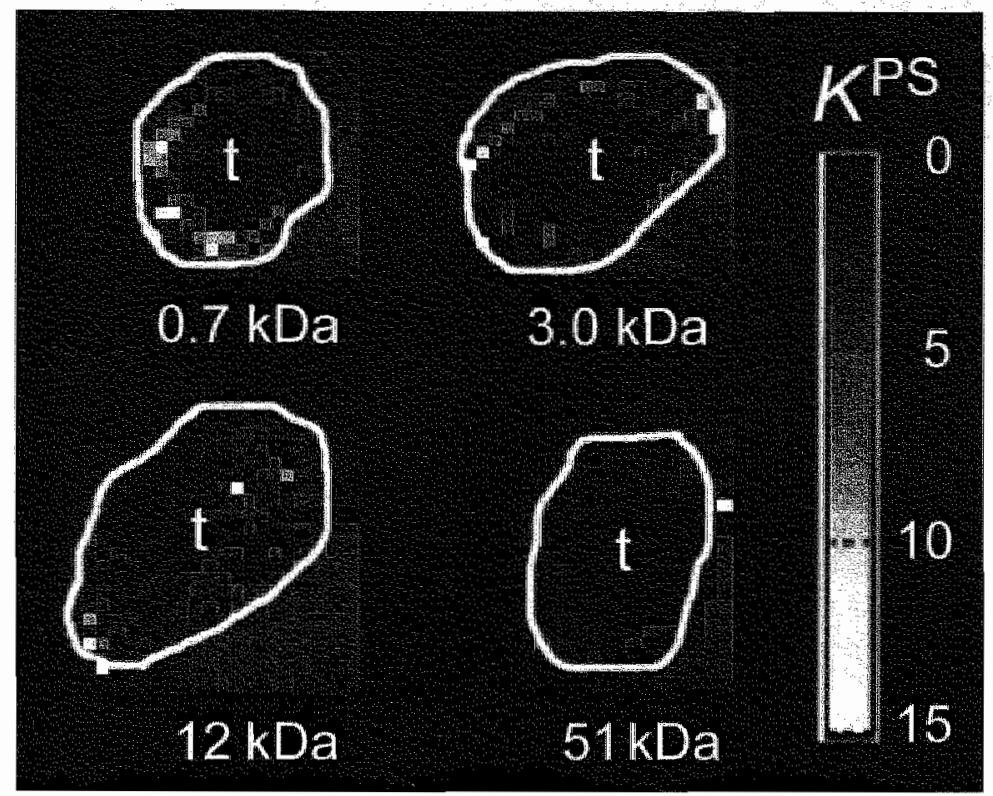

Figure 4. Color**-coded maps of $K^{\mathrm{PS}}$ values measured for the voxels in the central section through the tumor illustrate that use of smaller molecules result in higher $K^{\text {PS }}$ values, particularly in the tumor rim. Gray-scale voxels to the lower and upper right side of each tumor (in color) are adjacent tissue of the mice's left hind-limb; gray-scale voxels within the tumors were rejected from the kinetic analyses, due to either poor enhancement (possible necrotic areas) or excessive fit-errors.

** Color image is on back-cover (lower right)

\section{plvalues}

Our results showed that decreases in $f^{\text {pV }}$ in relation to the molecular weight of contrast agents followed a stepwise pattern, with a sudden decrease in $f^{p V}$ values (Figure 2c) between contrast agents with a molecular weight of $3.0 \mathrm{kDa}$ and those with a molecular weight of $12 \mathrm{kDa}$. Whole-tumor $\mathrm{f}^{\mathrm{PV}}$ values were between six and 10 times greater $(P=$ .01) for the 0.7-and 3,0-kDa agents than for the 12- and 51-kDa agents (Table 2).

Plasma fraction values showed little difference between the tumor rim and tumor core when low-molecular-weight contrast agents were used, and $f^{p V}$ values were at least four times greater in the tumor rim than in the tumor core when high-molecular-weight contrast agents were used. Average rim-core $f^{p v}$ ratios were $1.1 \pm 0.6,3.4 \pm 1.3,10.7 \pm$ 6.8 , and $4.7 \pm 4.0$ for the $0.7-, 3.0-12-$, and $51-k$ Da groups, respectively. Differences between tumor rim and tumor core $f^{P V}$ values showed a significant positive relationship ( $r$ $=0.5, P=.04$ ) with the molecular weights of the MR contrast agents.

Correlation coefficient values between molecular weight and $f^{P V}$ value were $-0.5(P=$ $.03)$ for the whole tumor, $-0.6(P=.01)$ for the tumor core, and $-0.5(P=.04)$ for the tumor rim. The differences in ranges in spatial distribution of $f^{\text {pV }}$ values showed an inverse relationship $(r=-0.6, P=.02)$ with the molecular weight of the contrast agent. 


\section{Discussion}

Earlier studies $(7,8)$ of tumor angiogenesis with human colon adenocarcinoma xenograft (LS 174T) have shown a heterogeneous and deficient vascular architecture, particularly at the tumor rim (i.e., lleading edge of the tumor). Angiogenesis in tumors induces hyperpermeability of vessels (B) and vasodilatation and expansion of the vasculature. Deficient remodeling leads to intermittent and rapidly changing heterogeneous flow (7) and leaves large open gaps (diameter, 400-600 nm) in the capillary wall (8). Development of antiangiogenesis treatments aims at inhibiting specific components of the angiogenic cascade (2). Consequently, specific microcirculatory changes induced by antiangiogenesis treatment will vary depending on the type of antianglogenesis agent used. Antiangiogenesis treatments may target endothelial cell proliferation, adhesion,

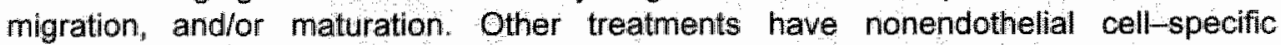
mechanisms of action, for example, they block activators of angiogenesis or interfere with tumor blood flow. The magnitude and spatial heterogeneity of the dynamic contrastenhanced MR imaging-derived parameters (i.e., $K^{P S}$ and $f^{P V}$ ) 16 days after tumor injection reflects these microcirculatory changes in tumor angiogenesis.

Interpreting our results requires a detailed understanding of contrast agent kinetics in dynamic contrast-enhanced MR imaging. $K^{P S}$ represents the rate of transfer of particles (i.e., leakage) from the blood to the interstitial space, and it is defined as the product of the flow $\left(F_{p}\right)$ and the extraction fraction $(E)$, which is calculated with the following equation: $E=\left[1-\exp \left(-P S / F_{p}\right)\right]$, where $P S$ is the permeability surface area product (14). With dynamic contrast-enhanced MR imaging, the basis for calculating this $K^{P S}$ value is determined by the leakage of an intravenously injected contrast agent. It is hypothesized that low-molecular-weight contrast agent molecules leak relatively easily from the blood through the interstitial matrix (away from the vessel) compared with high-molecularweight contrast agents. If this is true, $K^{\text {ps }}$ values, which are measured by using lowmolecular-weight contrast agents, will be influenced by the flow because their relatively fast rate of transfer to the interstitial space will increase as the blood flow increases and vice versa. In this situation (i.e., permeability surface area product > product of the flow). the extraction fraction approaches a value of 1 , and $k^{\mathrm{PS}}$ will be relatively large and approximate the tumor blood flow. Alternatively, high-molecular-weight contrast agents leak from the blood and move through the interstitial matrix with relative difficulty. Transfer to the interstitial matrix is limited by the relatively low intrinsic permeability and is independent of tumor blood flow. In this situation (i.e., permeability surface area product $<$ product of the flow), $K^{P S}$ values will approximate permeability surface area product best. The $K^{p s}$ of intermediate-molecular-weight contrast agents is influenced by both permeability surface area product and product of the flow (i.e., flow contamination). Our results show that the dynamic contrast-enhanced MR imaging-derived kinetic parameters (i.e., $K^{p S}$ and $f^{p V}$ ) increase in magnitude and spatial heterogeneity as the molecular weight of dendritic contrast agent decreases.

The assumption that $k^{P S}$ represents the microvessel permeability surface area product when high-molecular-weight contrast agents are used but represents the flow when lowmolecular-weight contrast agents are used is supported in the present study by two observations. First, the use of low-molecular-weight contrast agents resulted in increased magnitude and spatial heterogeneity of $K^{\text {PS }}$ compared with high-molecular-weight contrast agents. The increase in $K^{p S}$ magnitude, particularly $K^{p S}$ heterogeneity, is a result of the contribution of flow and an increase of permeability surface area product 
magnitude. Flow is known to be inhomogeneous in angiogenic regons $(7,8)$, wus, it yields a heterogeneous pattem.

Second, the use of low-molecular-weight contrast agents restuked h higher rim-core $K^{p s}$ ratios compared with high-molecular-weight contrast agents. This fonding supports the notion that $K^{p S}$ represents flow when low-molecular-weight contrast agents are used because angiogenesis-related increases in fow level and helerogeneity are most prominent in the rim of the tumor (7). Flow the thmor core is additionally reduced in comparison with the tumor rim because of relatively high local pressures. Elevated interstitial fluid pressure is a pathophysiologic characteristic of most human and experimental tumors. Interstitial fluid pressure increases toward the deeper core layers of the tumor by a large gradient, which is associated with lower pertusion or flow levels compared with the tumor rim (21).

On the contrary, the entire tumor is expected to exhibit a high degree of leakiness (h. $e_{*}$ high permeability surface area product) (8). As $K^{\mathrm{PS}}$ values for low-molecular-weight contrast agents are increased by flow level and high-molecutar-weight contrast agents are flow independent, the ratio between tumor rim and tumor core values of $k^{p s}$ will be higher for low-molecular-weight contrast agents than for high-molecular-weight contrast agents. Thus, relatively small rim-core differences in $K^{P S}$, as measured by using highmolecular-weight contrast agents, support the notion that $K^{\text {ps }}$ represents morovessel permeability surface area product. Surface area is not expected to be an important contributing factor for differences in $K^{\text {PS }}$ rim-core ratios measured by using either low- or high-molecular-weight contrast agents. The found quantitative relationshtp $K^{2 S}=$ $0.4 / \mathrm{MW}^{0.44}$ predicts that doubling of the molecular weight will reduce $k^{p s}$ values by approximately $25 \%$.

The hypothesis that $f^{v}$ values might be overestimated with rapidly diffusing lowmolecular-weight contrast agents when compared with high-molecular-weight contrast agents is supported by the inverse relationship found between molecular weight of the contrast agents, although the correct $f^{P V}$ remains unknown. Our results also suggest a threshold effect, with a marked decrease of $f^{p V}$ between 3.0 - and $12-k$ Da contrast agents. This step-wise decrease in $\mathrm{f}^{\mathrm{PV}}$ reflects the intravascular compartmentalization of molecules with a thigher molecular weight. The high spatial variations of $f^{p v}$ values may reflect the degree of heterogeneity of the microcirculation, which as mentioned previously, is a characteristic feature of tumor angiogenesis (7).

Measured $k$ values are in the same order of magnitude (mean $k$ value \pm standard deviation, $14.0 \mathrm{~mL} / 100 \mathrm{~cm}^{3} / \mathrm{sec} \pm 15.0$ ) as reported (10) for MR evaluations in a similar model with the use of gadopentetate dimeglumine. The positive relationship observed between tumor core $k$ values and molecular weight could have several explanations. Rates of diffusion through the interstitial matrix (i.e., away from the vessel) were lower for high-molecular-weight contrast agents than for low-molecular-weight contrast agents, which might enhance local interstitial concentration (i.e., near the vessel) and increase the chance to transfer back into the blood, resulting in slightly higher $k$ values for the high-molecular-weight contrast agents.

It should be noted that the parameters $k^{p s}$ and $k$ are related, but they are not equal. The reflux rate is the ratio of $K^{P S}$ and the extracellular extravascular space fraction. As lowmolecular-weight contrast agents will have a higher rate of diffusion through the extracellular extravascular space, so will this space belarger, which may compensate for the higher $K^{P S}$ value in the ratio for the reflux rate. Another explanation might be that higher rates of lymphatic drainage caused the smaller reflux to the blood compartment for the contrast agents with a low molecular weight. Finally, the dendritic agents were 
assumed to have no cellular interactions or matrix binding because the overall charge of the agents is slightly negative; nevertheless, processes like endocytosis and the unpredictable dependency on molecular weight could also have affected the apparent refiux rate. Contradictory $k$ values are reported $(10,11)$ for high-molecular-weight contrast agents (iron oxide particles and gadolinium conjugated with albumin) in the order of 10 times less than (mean $k$ value, less than 1 ) $k$ values measured with the $51-k D a$ dendritic agent (mean $k$ value, $19.1 \pm 11.2$ ). Thus, the positive relationship between tumor $k$ values and molecular weight may not be generalized or extrapolated for all agents with a high molecular weight than the $51-\mathrm{kDa}$ contrast agent. A possible explanation is that the $51-\mathrm{kDa}$ contrast agent is cleared by the kidneys, albeit at a slower rate than the contrast agents with a low molecular weight, whereas iron oxide particles and albumin conjugates are not cleared by the kidneys and circulate in high concentrations for prolonged periods of time $(10,11)$, thus preventing a reflux.

The results and implications of this study might be limited in several ways. The contrast agents that were used are experimental, and long-term effects are unknown because the mice were sacrificed immediately after imaging. The aim of this study was to correct for differences in excretion pathways and circulation properties by acquiring individual blood concentration time curves of the contrast agent for the arterial input function of each mouse.

Dose optimization studies were not performed. All contrast agents were administered with the same dose of gadolinium (i.e., $0.03 \mathrm{mmol} / \mathrm{kg}$ ). Traditionally, in MR studies for tumor angiogenesis, low molecular weight contrast agents (i.e., gadopentetate dimeglumine) were administered at a dose of $0.1 \mathrm{mmol} / \mathrm{kg}$, and high-molecular-weight contrast agents (i.e., albumin-bound gadolinium dimeglumine) were administered at a dose of $0.03 \mathrm{mmol} / \mathrm{kg}$. We chose to administer a gadolinium dose of $0.03 \mathrm{mmo} / \mathrm{kg}$. When T1-weighted MR measurements with short echo times are used, as in this study, the signal enhancement depends predominantly on the T1 shortening of the contrast agent, while the $\mathrm{T} 2{ }^{*}$ dependency is negligible. Alternatively $y_{\text {, }}$ one could decide to match the signal enhancement per gadolinium atom between the different dendrimers; however, such an approach would still not account for the expected lower tumor concentrations for the molecules with a higher molecular weight, which do have higher T1 relaxivities. In the future, combining $T 1$ relaxivities and known relationships between molecular weight and $K^{p S}$ "as found here, might prove to be a better approach to compare different contrast agents. For the application of the kinetic model, we calculated $T 1$ relaxation rates from the nonlinear relation of a gradient-echo pulse sequence between the pre-contrast $\mathrm{T} 1$ value, the pre-contrast signal intensity, and the contrastenhanced signal intensity. One might argue that the range of dendrimer relaxivities could have biased the correlation between $K^{P S}$ and molecular weight to be nonlinear by ignoring $\mathrm{T}^{*}$ effects. However, since we used a short echo time and obtained relatively low in wivo contrast agent concentrations, $12^{*}$ effects are negligible; thus, they are too small to have confounded the correlations found in this study.

Independent measurements to validate accuracy of the estimates of $K^{\mathrm{PS}}, k_{1}$ or $f^{\mathrm{PV}}$ were not performed here. Future studies might explore the accuracy of dynamic contrastenhanced MR imaging-derived parameters as measured by using low-, intermediate- or high-molecular-weight MR contrast agents in conjunction with validated absolute measurements of microcirculatory flow, permeability, surface area, and/or plasma volume. 
Practical application

Results of this study may aid in choosing the best contrast agent for dynamic contrastenhanced MR imaging. For example, these results may be used to evaluate effects of antiangiogenesis and other antitumor treatments. Depending on the biologic effects of the treatment strategy, high-molecular-weight contrast agents might be preferred over low-molecular-weight contrast agents in the evaluation of reductions in microvessel permeability and vascular volume. Low-molecular-weight contrast agents might be more applicable in the evaluation of therapeutic reductions in magnitude and heterogeneity of microcircullatory blood flow. 


\section{References}

1. Carmeliet $P$, Jain RK. Angliogenesis in cancer and other diseases. Nature 2000; 407:249-257.

2. Griffioen $A W_{i}$ Motema $G$. Angiogenesis: potentials for pharmacologic intervention in the treatment of cancer, cardiovascular diseases, and chronic inflammation. Pharmacol Rev $2000 ; 52: 237-268$.

3. McDonald DM, Choyke PL. Imaging of angiogenesis: from microscope to clinic Nat Med 2003, Q:713-725.

4. Padhani AR. Dynamic contrast-enhanced MRI in clinical oncology: current status and future directions. J Magn Reson Imaging 2002; 16:407-422.

5. van Dije $C F$, Erasch RC, Roberts $T P_{\text {n }}$ et al. Mammary carcinoma model: correlation of macromolecular contrast-enhanced MR imaging characterizations of tumor microvasculature and histologic capillary density. Radiology $1996 ; 198: 813-818$.

B. Tofts PS, Brix G, Buckley DL, at al. Estimating kinetic parameters from dynamic contrastenhanced T(1) weighted MRI of a diffusable tracer: standardized quantities and symbols. J Magn Reson Imaging 1999; 10:223-232.

7. Patan $S$, Munn $L L$, Jain RK. Intussusceptive microvascular growth in a human colon adenocarcinoma xenograft: a novel mechanism of tumor angiogenesis. Microvasc Res 1996; 51:260-272.

8. Hobbs SK, Monsky WL, Yuan $F_{1}$ et al. Regulation of transport pathways in tumor vessels: role of tumor type and microenvironment. Proc Natl Acad Sci U S A 1998; 95:4607-4612.

9. Choyke PL, Dwyer AJ, Knopp MV. Functional tumor imaging with dynamic contrast-enhanced magnetic resonance imaging. J Magn Reson Imaging 2003; 17:509-520.

10. de Lussanet QG, Backes WH, Griffioen AW, wan Engelshoven JM, Beets-Tan RG. Gadopentetate dimeglumine versus ultrasmall superparamagnetic iron oxide for dynamic contrast-enhanced MR imaging of lumor angiogenesis in human colon carcinoma in mice. Radiology $2003 ; 229: 429-438$.

11. Turetschek $K$, Huber $S$, Floyd $E$, et al. MR imaging characterization of microvessels in experimental breast tumors by using a particulate contrast agent with histopathologic correlation. Radiology 2001; 218:562-569.

12. Roberts TP, Turetschek $K$. Preda $A$, et al. Tumor microvascular changes to anti-angiogenic treatment assessed by MR contrast media of different molecular weights. Acad Radiol 2002; 9(supp/2):S511-S513.

13. Bhujwalla ZM, Artemov $D$, Natarajan $K$, Solaiyappan M, Kollars $P$, Kristjansen PE. Reduction of vascular and permeable regions in solid tumors detected by macromolecular contrast magnetic resonance imaging after treatment with antiangiogenic agent TNP-470. Clin Cancer Res 2003; 9:355-362

14. Daldrup $H$, Shames $D M$, Wendland $M$, et al. Correlation of dynamic contrast-enhanced MR imaging with histologic tumor grade: comparison of macromolecular and small-molecular contrast media. AJR Am J Roentgenol 1998; 171:941-949.

15. Kobayashi H. Kawamoto $\mathrm{S}$, Jo SK, Bryant HL, Jr, Brechbiel MW, Star RA. Macromolecular MRI contrast agents with small dendrimers: pharmacokinetic differences between sizes and cores. Bioconjug Chem 2003; 14:388-394.

16. Langereis S, de Lussanet QG, van Genderen MH, Backes WH, Meijer EW. Multivalent dendritic contrast agents based on Gd-DTPA-terminated poly(propylene imine) dendrimers for magnetic resonance imaging. Macromolecules 2004; 37:3084-3091.

17. Hummelen $\mathrm{J} \mathrm{C}$, vanDongen $\mathrm{JL}$, Mejer EW. Electrospray mass spectrometry of polyípropylene imine) dendrimers: the issue of dendritic purity or polydispersity. Chem Eur $\mathrm{J} 1997 ; 3: 1489-1493$.

18. Bosman AW, Janssen HM, Meijer EW. About dendrimers: structure, physical properties, and applications. Chem Rev 1999; 99:1665 1688.

19. Dialdrup HE, Shames DM, Husseini W, Wendland MF, Okuhata $Y$, Brasch RC. Quantification of the extraction fraction for gadopentetate across breast cancer capillaries. Magn Reson Med $1998 ; 40.537-543$.

20. Zar JH. Power and sample size in correlation. In: Zar $\mathrm{JH}$, eds. Biostatistical analysis. 3rd ed. Upper Saddle River, NJ: Prentice-Hall International, 1996; 379-381.

21. Boucher $Y$, Baxter LT, Jain RK. Interstitial pressure gradients in tissue-isolated and subcutaneous tumors: implications for therapy. Cancer Res 1990; 50:4478- 4484. 


\title{
Chapter 4
}

\section{Dynamic contrast-enhanced MRI at 1.5 Tesla with gadopentetate dimeglumine to assess angiostatic effects of anginex in mice}

\author{
European Journal of Cancer, 2004 May;(40)8:1262-1268
}

Quido G. de Lussanet, Regina G.H. Beets-Tan, Walter H. Backes, Daisy W.J. van der Schaft, Jos M.A. van Engelshoven, Kevin H. Mayo, Arjan W. Griffioen.

\section{ABSTRACT}

\section{PURPOSE}

The purpose of this study was to evaluate the effects of anginex on tumor angiogenesis assessed by dynamic contrast-enhanced magnetic resonance imaging (DCE-MRI) on a clinical 1.5 Tesla MR system and with the clinically available contrast agent gadopentetate dimeglumine.

\section{MATERIALS AND METHODS}

C57BL/6 mice carrying B16F10 melanomas were treated with anginex, TNP-470 or saline. Tumor growth curves and microvessel densities (MVD) were recorded to establish the effects of treatment. DCE-MRI was performed on day 16 after tumor inoculation, and the endothelial transfer coefficients of the microvessel permeability surface-area product $\left(K^{P S}\right)$ were calculated using a two-compartment model.

\section{RESULTS}

Both anginex and TNP-470 resulted in smaller tumor volumes $(p<0.0001)$ and lower MVD $(p<0.05)$ compared to saline. Treatment with anginex resulted in a $64 \%$ reduction $(p<0.01)$ of tumor $K^{P S}$ and TNP-470 resulted in a $44 \%$ reduction $(p=0.17)$, compared to saline.

\section{CONCLUSION}

DCE-MRI with a clinically available, low molecular weight contrast agent can therefore be used to evaluate the angiostatic effects of anginex and TNP-470 on tumor angiogenesis. 


\section{Introduction}

Angiogenesis is essential to normal bodily processes such as embryogenesis and wound healing, but it is also a key regulator of various pathological disorders, including tumor growth, arthritis, endometriosis, atherosclerosis and diabetic retinopathy $(1,2)$. The targeting of blood vessels is considered a potentially useful anticancer strategy, primarily because endothelial cells are more accessible than other cells to pharmacological agents delivered vila the blood, and because they are genetically stable and thus not easily mutated into drug-resistant variants. Anti-angiogenesis therapy could be a promising modality in the future, either as monotherapy or in combination with conventional cancer therapy (1). The search for new inhibitors of angiogenesis with reduced toxicity, improved potency, stability, selectivity and ease of delivery is an important field of research. A revolutionary development in this search has been the design of small, cytokine-like peptides that contain only selective domains of traditional anti-angiogenesis agents in order to create well-defined antitumor agents that focus on specific angiogenesis processes. This approach produced anginex, which was designed according to the three-dimensional, $\beta$-sheet structure of $\alpha$-chemokines such as platelet factor 4 and interleukin 8 (3).

In vitro anginex acts by blocking the adhesion and migration of activated endothelial cells (3), leading to cell detachment, a process that is called anoikis, and the subsequent induction of apoptosis. In vivo angiinex targets angiogenically activated (tumor) endothelial cells and inhibits angiogenesis, ultimately leading to anticancer activity in mice, both as a single therapy and in combination with chemotherapy (4), with no toxic effects observed thus far (5).

Generally accepted standards of reference for evaluating angiogenesis activity and the effects of treatment are the histological assessment of microvessel density (MVD) and the measurement of circulating angiogenesis factors. The reliability of these surrogate markers is subject to considerable dispute. New techniques for monitoring the effects of anti-angiogenesis treatment that are less or non-invasive are currently under investigation (6). Dynamic contrast-enhanced, $T_{4}$-weighted magnetic resonance imaging (DCE-MRI) (6-9) is a non-invasive imaging technique that can be used to derive microcirculation properties that mark tumor anglogenesis $(7,10)$. Various models have been investigated that relate changes in signal intensity over time as measured with DCE-MRI to tumor activity. The most effective models are those that convert the measured changes in signal intensity into changes in contrast-agent concentration and subsequently apply a pharmacokinetic two-compartment analysis. Two-compartment analysis yields parameters for a plasma fraction $\left(f^{P V}\right)$ within the tumor and a rate of exchange between the blood and interstitial compartments (i.e. a transfer coefficient, $K^{P S}$ ) (8). Applications of DCE-MRI in animal MR systems and with experimental, largemolecular contrast agents effectively showed reductions in $f^{P V}$ and $K^{P S}$ in the rim of solid tumors after treatment with anti-angiogenesis agents (8) including the fumagillin derivative TNP-470 (10). Currently, such large-molecular contrast agents are unsuitable for DCE-MRI in man. Preliminary evidence suggests that DCE-MRI studies are reproducible when using clinical 1.5 Tesla MR systems and clinically approved, low molecular weight contrast agents $(11,12)$.

It was hypothesized that the anti-angiogenesis mechanism of anginex will lead to a decreased tumor MVD and to maturation of the vasculature, which would be expected to reduce the tumor transfer coefficient. The aim now was therefore to evaluate the antiangiogenesis effects of the angiostatic compounds anginex and TNP-470 on tumor 
angiogenesis assessed by DCE-MRI on a clinical 1.5 Tesla MR system and with the clinically available contrast agent gadopentetate dimeglumine.

\section{Materials and methods}

\section{Mouse tumor model}

The local efhical review committee approved the animal experiments. B16F10 melanoma cells (provided by $\mathrm{Dr} \mathrm{J}$. Fidller, Texas) were cultured in minimal essential medium (MEM) with Hank's salts, 10\% fetal calf serum (BioWhittaker, Verviers, Belgium), $50 \mathrm{lU} / \mathrm{ml}$ penicillin (ICN, Aurora, Ohio) and $50 \mathrm{UU} / \mathrm{ml}$ streptomycin (Seva, Heidelberg, Germany), MEM vitamins, 2-mM B-glutamine, non-essential amino acids and $2 \mathrm{mmol}$ sodium pyruvate. Cells were harvested with trypsin (0.125\%) (DIFCO, Detroit, Michigan). At day 0, 6-week-old C57BL/6 mice $(n=34)$ (obtained from Charles River, Heidelberg, Germany) were inoculated subcutaneously in the right flank with $2 \times 10^{5}$ B16F10 cells. Once tumors became visible, between day 6 and 9 , each one was measured daily with a caliper and tumor volumes were recorded, calculated as width ${ }^{2} \times$ length $\times 0.52$ (13). Mice were randomized and treated with anginex $(n=14)$, the established angiogenesis inhibitor TNP-470 $(n=9)$ or saline $10.9 \% \mathrm{NaCl}$ solution) alone $(n=11)$, using previously optimized drug dosages and modes of administration $(3,14)$. Anginex (3), dissolved in saline, was administered ( $6 \mathrm{mg} / \mathrm{kg}$ body wt per day) from day 0 through a surgically implanted Alzet osmotic minipump (Durect, Cupertino, CA). TNP. 470 (provided by Takeda Chemical Industries, Osaka, Japan), dissolved in saline with $3 \%$ ethanol and $5 \%$ glucose (just before treatment diluted from a stock of $30 \mathrm{mg} / \mathrm{ml}$ in dimethyl sulphoxide), was administered by intraperitoneal injection (60 mg/kg body wt) every other day. Treatment with TNP-470 was started from day 9 to allow the formation of an early-stage tumor and prevent toxic effects due to prolonged treatment.

\section{$M R I$}

On day 16, 13 mice from the population described above, with volume-matched tumors to exclude tumor size-dependent differences (10), were selected for MRI (saline, $n=5$; anginex, $n=5$; TNP-470, $n=3$ ). Before imaging the mice were anesthetized by subcutaneous injection of $100-\mathrm{mg} / \mathrm{kg}$-body wh ketamine (Nimatek; Eurovet, Bladel, The Netherlands) and $10-\mathrm{mg} / \mathrm{kg}$ xylazine (Sedamun; Eurovet), and the subcutaneous osmotic minipumps were removed. MRI was as previously reported (12), using a circular surface coil (diameter, $5 \mathrm{~cm}$ ) and a 1.5 Tesla system (Intera; Phillps, Best, The Netherlands). A $T_{2}$-weighted acquisition [multislice turbo-spin echo, echo-train length 28 ,

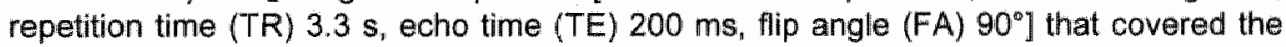
tail-end half of the mouse was used for tumor delineation, with 16 transverse sections, a field of view of $44 \times 64 \times 32 \mathrm{~mm}$ (anterior-posterior $\times$ left-right $\times$ feet-head), matrix dimensions of $88 \times 128$, pixel size $0.5 \times 0.5$, and slice thickness $2.0 \mathrm{~mm}$. Six $T_{1}$-weighted acquisitions [three-dimensionall fast-field echo (3D FFE), TR $50 \mathrm{~ms}$, TE $7 \mathrm{~ms}$ ] were performed with different $\mathrm{FA}\left(2^{\circ}, 5^{\circ}, 10^{\circ}, 15^{\circ}, 25^{\circ}\right.$ and $\left.35^{\circ}\right)$ to determine the local $T_{1}$-relaxation times before contrast enhancement (15). Subsequently, a $T_{1}$-weighted, dynamic contrastenhanced series of 50 scans (3D-FFE: TR $50 \mathrm{~ms}$ TE $7 \mathrm{~ms}$, FA $35^{\circ}$ ), 39 s/volume acquisition) was conducted, with 16 transverse sections, a field of view of $44 \times 64 \times 32$ $\mathrm{mm}$, matrix dimensions of $88 \times 128$, a slice thickness of $4.0 \mathrm{~mm}$, and pixel size $0.5 \times 0.5$ $\mathrm{mm}$. Adjacent sampled sections were $2.0 \mathrm{~mm}$ displaced and subsequently interpolated to $162.0 \mathrm{~mm}$-thick sections during reconstruction. Gadopentetate dimeglumine 
(Magnevist; Schering AG, Berlin, Germany; 0.1-mmol/kg body wt) was manually infused into the tail vein with $15 \mu$ of saline to "flush" the injected volume. Administration of the total injection volume was standardized by timing the (slow) injection duration of $30 \mathrm{si}$ to prevent the high concentrations that might cause susceptibility artifacts. Injection of contrast agent was started at the onset of the fifth scan of the dynamic series. The temperature in the magnet bore was maintained at $28^{\circ} \mathrm{C}$.

\section{Analysis of MRl data}

The $T_{2}$-weighted anatomical acquisitions were used to locate and delineate the tumor from the surrounding tissue and edema. A region of interest was manually drawn around the whole tumor volume in the central slice though the tumor. This region of interest was used for further analysis. Regions of interest for the tumor rim were defined as the approximately 1 to $2 \mathrm{~mm}$-thick peripheral zone of strong contrast enhancement $(7)$ in the dynamic $T_{1}$-weighted series; the tumor core was the remaining central region.

Kinetic analysis of the images was performed as described previously (12). Local timeaveraged $T_{1}$ relaxation rates of the precontrast images $\left(R_{10}\right)$ and the postcontrast image $\left(R_{1}\right)$ signal-intensity changes over time (Figure 1) were used to determine the time course of the plasma concentration in the aorta, representing the arterial input function and tissue concentration in the tumor.

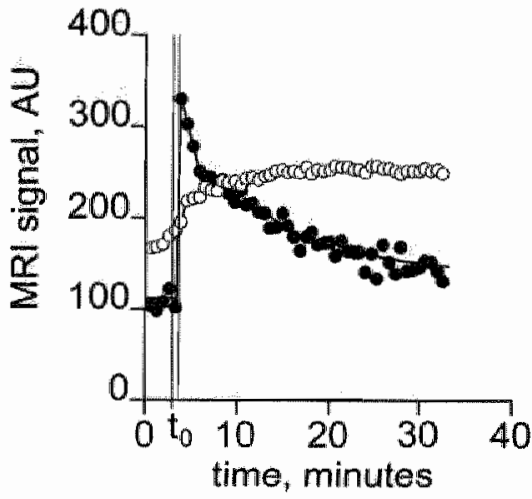

Figure 1. Signal intensity ( $\mathrm{SI}_{\text {" }}$ arbitrary units) time courses (•) measured in the aorta show immediate signal intensity increases after injection (to) of the contrast agent gadopentetate dimeglumine, followed by a bi-exponential decline. No re-circulation peak is observed, because the duration of contrast agent injection and dynamic volume acquisitions were longer than blood-circulation times in mice. Average signal intensity time courses measured in the tumor (o) show more gradual signal intensity increases than in the blood which diminish again approximately 15 minutes post injection (to).

Individual input functions were used to correct for small variations in the manual injection of the contrast agent, intrinsic circulation properties and contrast elimination. The plasma $R_{1}(t)$ time course was fitted to a bi-exponential function. The constant (i.e. $T_{\text {y }}$ relaxivity) describing the ratio of $R_{f}$ change to contrast-agent concentration has been found (12) to be $5.6 \mathrm{mM}^{-1}$ per's by in vitro measurements of mouse plasma (with citrate buffer) concentrations at 1.5 Tesla. A pharmacokinetic, two-compartment bidirectional exchange model $(12,16,17)$ was used to determine the tumor endothelial transfer coefficient $\left(K^{P S}\right)$, reflux coefficient $(k)$ and fractional plasma volume $\left(f^{P V}\right)$ for each voxel. $k^{P S}$ and $k$ were expressed as $\mathrm{m} / / \mathrm{min}$ per $100 \mathrm{~cm}^{3}$ of tissue and $\mathrm{f}^{\mathrm{PV}}$ were expressed as $\mathrm{m} / 1 / 100 \mathrm{~cm}^{3}$ of tissue. It was previously shown (12) that for this model, using gadopentetate dimeglumine, the $K^{\text {PS }}$ is of main interest for indexing angiogenesis activity. Color-coded $K^{P S}$ maps, overlaid on the corresponding $T_{y}$-weighted image (as illustrated in Figure 2), were used for defining regions of interest for which $K^{P S}, f^{P V}$ and $k$ were spatially averaged for the whole tumor, the tumor rim and the core. The data 
processing was performed in the MATLAB programming environment (The MathWorks, Natick, MA).

\section{MVD}

On day 16, all mice were killed by cervical dislocation under narcosis and tumors were excised. From these tumors, axial cryosections $(5 \mu \mathrm{m})$ corresponding to the MRI sections were cut and stained immunohistochemically using anti-CD31 antibody (followed by goat anti-rat PO and DAB development). MVD was evaluated as described previously (18). In brief, two independent observers assessed MVD by counting the number of vessels in three high-power fields randomly selected within a section. In addition. MVD maps were generated for the tumors of mice that underwent DCE-MRI by counting blood vessels in microscopic fields of $0.45 \times 0.45 \mathrm{~mm}^{2}$ (magnification $250 \times$ ) for a full tumor section.

\section{Statistical analysis}

Tumar growth curves for the anginex. TNP-470 and saline treatment groups were compared by two-way analysis of variance. MVD, $K^{P S}, k_{\text {, }}$ and $\mathrm{f}^{\mathrm{PV}}$ for the different treatment groups, and rim and core MVD in the MVD maps, were compared by unpaired, two-sided Student $t$-tests. Intratumor rim and core $K^{p s}, k$ and $f^{p V}$ were compared for the treatment and control groups by paired, two-sided Student t-tests. $K^{p s}$, $k_{\text {, }}$ and $\mathrm{f}^{\mathrm{V} V}$ for the different treatment groups were also evaluated using the Mann-Whitney test to account for possible non-normal distribution of variables. Statistical analyses used commercial software (SPSS 10.0.5" SPSS Inc. "Chicago, IL) and the level of significance was set at $P<0.05$.

\section{Results}

\section{Antitumor activity of TNP-470 and anginex}

Anginex inhibited tumor growth by $68 \%$ ( $p<0.0001$ ), as compared to growth in saline control mice (Figure 3a). TNP-470 showed $65 \%$ inhibition $(p<0.0001)$ of tumor growth, as compared to saline control mice (Figure 3a). Tumor MVD was reduced by approximately $50 \%$ for both anginex and TNP-470 ( $p<0.05$; Figure $3 b)$. Toxic effects were observed after TNP-470 treatment. but not after anginex (data not shown). $\mathrm{K}^{\mathrm{PS}}$ for tumor vessels by MRI in mice treated with anginex

Clear tumor delineation and differentiation from adjacent muscle tissue and edema was possible with the $T_{2}$-weighted acquisition (Figure $2 a$ ). $K^{p s}$ in tumors, calculated using $T_{1-}$ weighted DCE-MRI measurements, showed a decrease of $64 \% \quad(p<0.01)$ in anginextreated mice as compared with $K^{P S}$ in tumors of saline control mice (Table 1). Reductions in $K^{P S}$ were most statistically significant in the rim of the tumor $(66 \%$, $p<0.003$ ). The tumor core showed a $67 \%$ reduction in $K^{\mathrm{PS}}$. which almost reached statistical significance $(p=0.05$; Table 1). MRI on tumors in mice treated with TNP-470 showed decreases in $K^{P S}$ in the whole tumor of $44 \%(p=0.17)$, in the rim of $43 \%(p=0.16)$ and in the core of $43 \%(p=0.16)$ (Table 1). No significant differences $(p>0.5)$ were observed between the $K^{+5}$ of mice treated with anginex or TNP-470.

For the tumors in mice that were selected for DCE-MRI, MVD measurements were performed for the full area of the axial cryosections through the center of the tumor in correspondence to the MRI sections. MVD maps (Figure 2c) were aligned and compared with their respective $K^{P S}$ maps shown in pseudo-color (Figure 2b). A close similarity was observed between MVD and $K^{\mathrm{PS}}$ for individual tumors, of which an example from a 
saline control mouse is presented in Figure 2. In addition, MVD maps of the saline control tumors show higher values in the rim of the tumor $(36 \%$ higher, $p<0.002)$ than in the tumor core. This difference was only statistically significant in saline control mice; in anginex-treated mice the difference was non-significantly $(\mathrm{p}=0.3)$ reduced by $11 \%$. This finding was in line with the observed $K^{P S}$, with a stronger rim versus core difference $(p<0.003)$ in saline control mice than in anginex-treated mice $(p=0.09)$ (Table 1).
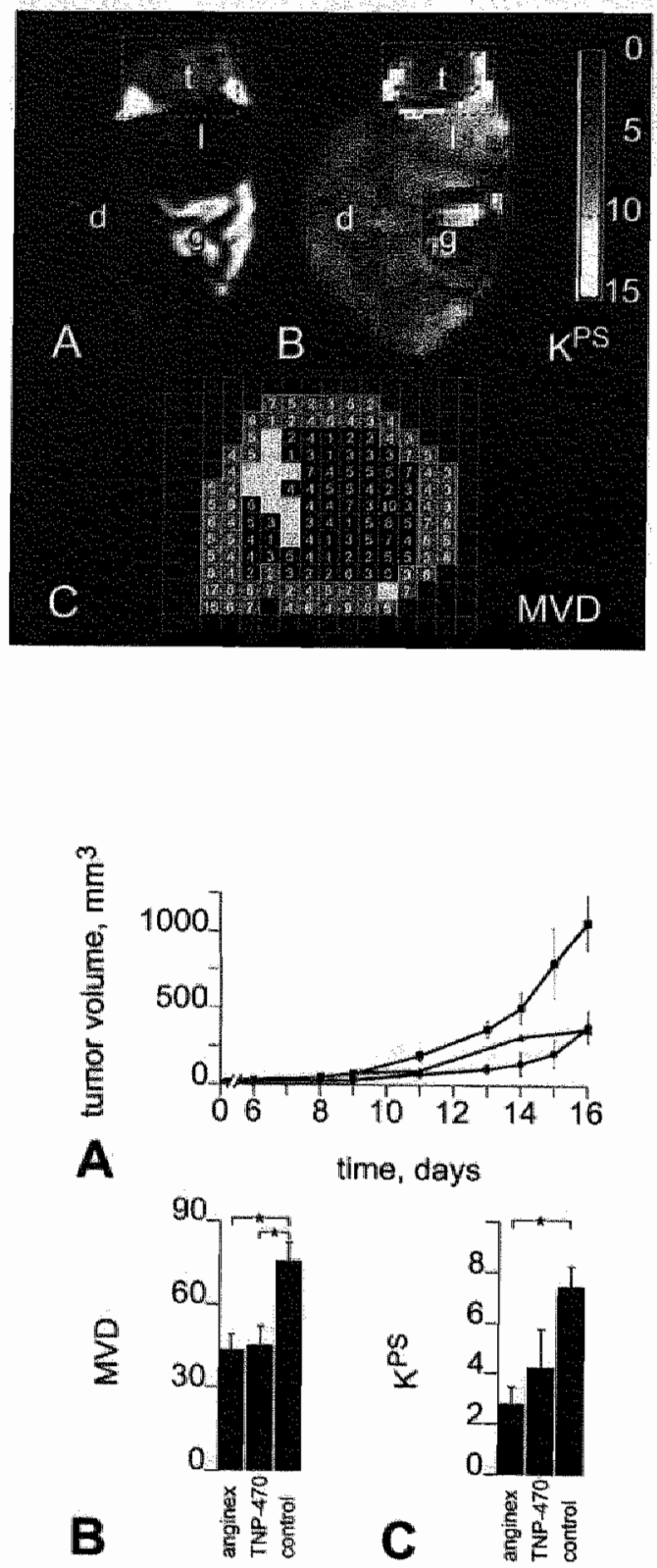

Figure 2. (a) On the axial $T_{2}$-weighted image of the mouse, tumor (t) tissue (grey) and oedema (white) are clearly discernible from adjacent muscle tissue (nearly black) of the hind leg $(\|)$, within the grey dotted box; $d=$ dorsal side, $g=$ gut. (b) Maps of the transfer coefficient $\left(K^{P S}, \mathrm{mll}\right.$ $\min ^{-4}$ per $100 \mathrm{~cm}^{3}$ of tissue), in pseudo-color ${ }^{4 *}$ (scale on the right) are superimposed on the $T_{1}$-weighted image. Areas of low $K^{P S}$ values correspond with darker areas in the tumor on the $T_{2}$-weighted image and were identified as necrotic regions on histologic sections of the tumor, in the same axial orientation as the MRI slices. (c) Maps of the microvessel density (MVD, vessels per $0.2 \mathrm{~mm}^{2}$ ), of the CD-31 stained histology section, shows a higher mean MVD for the tumor rim (red ${ }^{* *}, M V D=5.2 \pm 2.6$ [SD]) compared to the core (black, MVD $=3.8$ \pm 1.9 [SD]), as well as necrotic regions (grey fields).

*ik Color image is on the back-cover (upper left)

Figure 3. (a) Tumor growth curves show significant inhibition of tumour growth for mice treated with anginex $\left(\right.$ started at day $0_{n}$ $p<0.0001$ ) and TNP-470 ( $\Delta$, started at day 9 , $p<0.0001$ ) in comparison with saline $(\mathbf{m})$. For clarity, variations in tumor measurements the standard error [SE] was typically $20 \%$ in all groups) are only shown for the anginex and the saline groups. (b) Microvessel density (MVD) vallues for tumours of mice treated with anginex, TNP-470 or saline alone (* $p<0.05)$. (c) Transfer coefficient $\left(K^{P S}, \mathrm{mll} \mathrm{min}^{-4} 100^{-3}\right.$ of tissue) values [ \pm SE] measured in the rim of the tumour, showing similar reductions as compared with results from the MVD measurements ( $\left.{ }^{*} \mathrm{p}<0.003\right)$. 
MRll-based PV measurements suggested a decrease of plasma fractions, particularly for the tumor rim, in treated mice as compared to saline control mice (Table 1). No significant differences in $f^{p}$ were observed between the saline control mice and mice treated with anginex $(p=0.15)$ or TNP-470 $(p=0.3)$. Rim vs, core differences in $f^{p v}$. although not statistically significant, were slightly stronger in saline control mice $(p>0.1)$ than in anginex-treated mice $(p>0.3)$ (Table 1).

MRI-based measurements of the reflux coefficients $(k)$ revealed no significant differences between saline control mice and mice treated with anginex $(p>0.9)$ or TNP. 470 ( $p>0.5$ ) Table 1). Also, no particular trends were observed in differences between rim and core $k$ for saline control $(p>0.08)$ mice, anginex- $(p>0.5)$ or TNP-470- $(p>0.7)$ treated mice.

Table 1 DCE-MRI parameters for tumors treated with angiostatic agents

\begin{tabular}{|c|c|c|c|c|}
\hline$K^{p s}$ & $\begin{array}{l}\text { Tumor } \\
\text { Rim }^{\circ} \\
\text { Core }\end{array}$ & $\begin{array}{l}\text { saline } \\
5.5 \pm 0.9^{\circ} \\
7.4 \pm 0.8 \\
3.0 \pm 0.6\end{array}$ & $\begin{array}{l}\text { anginex } \\
2.0 \pm 0.4 \\
2.8^{*} \pm 0.7 \\
1.3 \pm 0.2\end{array}$ & $\begin{array}{l}\text { TNP-470 } \\
3.1 \pm 1.2 \\
4.2 \pm 1.5 \\
1.7 \pm 0.5\end{array}$ \\
\hline$f^{P W}$ & $\begin{array}{l}\text { Tumor } \\
\text { Rim } \\
\text { Core }\end{array}$ & $\begin{array}{r}8.6 \pm 3.9 \\
14.4 \pm 6.8 \\
4.3 \pm 2.4\end{array}$ & $\begin{array}{l}1.9 \pm 1.0 \\
2.1 \pm 0.5 \\
0.9 \pm 0.7\end{array}$ & $\begin{array}{l}7.7 \pm 6.7 \\
8.3 \pm 7.0 \\
5.8 \pm 5.2\end{array}$ \\
\hline$k$ & $\begin{array}{l}\text { Tumor } \\
\text { Rim } \\
\text { Core } \\
\end{array}$ & $\begin{array}{r}10.0 \pm 3.4 \\
10.8 \pm 3.7 \\
7.8 \pm 2.7 \\
\end{array}$ & $\begin{array}{l}11.6 \pm 4.2 \\
10.1 \pm 4.3 \\
13.7 \pm 5.4\end{array}$ & $\begin{array}{r}5.5 \pm 2.0 \\
6.5 \pm 2.9 \\
6.8 \pm 2.1 \\
\end{array}$ \\
\hline Note: & \multicolumn{4}{|c|}{ 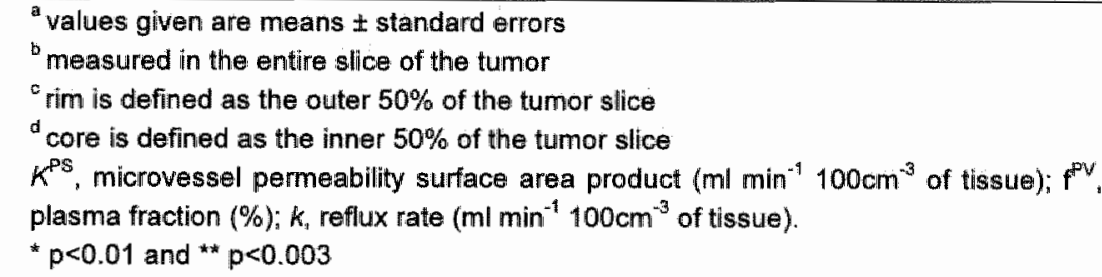 } \\
\hline
\end{tabular}

\section{Discussion}

The results of this study demonstrate that effects of the angiostatic compounds anginex and TNP-470 on tumor angiogenesis can be assessed by DCE-MRI on a clinical 1.5 Tesla MR system and with the clinically available contrast agent gadopentetate dimeglumine. The novel cytokine-like peptide anginex and the established angiogenesis. inhibitor TNP-470 were used to treat cancer in a mouse solid tumor model. TNP -470 is a validated angiogenesis inhibitor and was used here as a positive control according to a previously optimized treatment regimen (14). Both anginex and TNP-470 are examples. of angiogenesis inhibition through direct action on endothelial cells, which has been shown as yet to be the most effective strategy for angiogenesis inhibition as a treatment for cancer $(1,4)$.

In animal studies, the angiostatic effects of compounds are generally assessed by measuring MVD or by detecting circulating angiogenesis factors. MVD is a controversiall measure for prognosis because (i) it is a non-physiological variable that cannot differentiate functional from non-functional and non-perfused blood vessels, and (ii) it is subject to considerable (inter- and intra-observer) variation. The latter variability made 
an international methodological consensus for the assessment of MVD necessary (19). The detection of eirculating angiogenesis factors may similarly suffer from excessive variations, but its major limitation is that it is not yet known what responses (increases or decreases) to expect from specific inhibitors. These considerations necessitate the search for other, preferably non-invasive, surrogate markers. Therefore, great attention is directed toward the development of functional imaging techniques, such as positronemission tomography, computed tomography, MRI and other non-invasive imaging methods (6), which may localize sites of angiogenesis and offer functional data in animal angiogenesis models and in man. The successful use of MRI has been demonstrated in this study.

More importantly, a clinical MR system and a widely available, clinical low molecular weight MR contrast agent proved to be applicable for monitoring the effects of antiangiogenesis agents and to differentiate the amount of angiogenesis activity within a tumor. Our finding of reduced transfer coefficients after treatment with TNP-470, measured at 1.5 Tesla and with a low molecular weight contrast agent, is in line with earlier reported (10) reductions in vascularity and permeabillity measured in another mouse tumor model with a dedicated, high-field, animal MR system and a largemolecular, experimental contrast agent.

It is important to consider the molecular size of the contrast agent used. First, small- and large-molecular contrast agents may differ greatly in their respective blood concentration changes over time, which may have implications for the required acquisition times and the speed of contrast-agent injection in the dynamic contrast-enhanced series. Blood concentration changes cain be expected to be rather larger for low molecular weight clinical contrast agents, such as gadopentetate dimeglumine, which are rapidly cleared from the circulation, than for large-molecular experimental contrast agents, which have relatively slow clearance rates. To date, however, no large-molecular contrast agent that is suitable for DCE-MRI has reached approval for clinical use. Good but not perfect correlations were shown between MVD and $K^{\text {ps }}$ using the gadopentetate dimeglumine contrast agent in a mouse solid tumor model (12). Based on that study, as well as the results of our dynamic contrast-enhanced measurements (Figure 1), we assume our temporall resolution to be sufficient for deriving reliable $K^{P S}$. As is shown in figure 1 , with a temporal scan resolution of $39 \mathrm{~s}$ and a relatively slow speed of injection, sufficient samples were obtained in the rising part of the tissue signal-intensity curve and the declining part of the vascular-input function.

A second reason for regarding the molecular size of the contrast agent used is to explore which physiological properties are reflected by the $K^{\mathrm{F}} \mathrm{S}$. For low molecular weight contrast agents the $K^{P S}$ represents the permeability surface-area product (PS) that is to some extent contaminated by flow, as has been shown in a rat tumor model (20). Both permeability and flow are suitable microcirculatory properties for characterizing the status of tumor vascularization and are likely to decrease during vascular normalization. Reductions in $K^{P S}$ after treatment with anginex and TNP -470 , which were well correlated with reductions in MVD, could have resulted from reduced microvessel permeability, reduced microvessel surface area (per volume of tissue), reduced blood flow, or a combination of these (8). A study (21) using a rat breast cancer model showed significant reductions in $K^{P S}$ with the use of llarge-molecular contrast agents after antiangiogenesis treatment with anti-vascular endothelial growth factor (VEGF) antibody, but failed to measure these reductions in $K^{P S}$ with the use of low molecular weight contrast agents. The anti-angiogenesis effects of anti-VEGF antibody primarily target the initiation of angiogenesis, which involves the induction of microvessel permeability by VEGF $(1,2)$, 
whereas TNP-470 and anginex directly target endothelial cells involved in new vessel formation $(1-5,14)$. Therefore, the success of using low molecular weight agents to measure the effects of anti-angiogenesis treatment might depend on their biological effects. Other reasons might lie in the details of the applied MRI protocol. For example, we feel that, to monitor the concentration changes, a low rate of contrast-agent injection; rather than high-speed bolus injection (as used in other studies (21), is essential for rapidly diffusing, small contrast-agent molecules.

The finding that anti-anglogenesis compounds eliminated the rim-core difference in $K^{p s}$ supports the suggestion that treatment with angiostatic compouinds can normalize the angiogemesis phenotype of the tumor blood vessels, since angiogenesis is generally most prominent in the rim of a tumor $(1,2)$.

Variations in $f^{p V}$ were too great to reflect significant reductions in blood volume, which may have resulted from the fast diffusion properties of the low molecular weight contrast agent. The diagnostic value of the $f^{P V}$ might be better with the future development of large-molecular contrast agents that are safe for injection in man.

The diagnostic value of the reflux parameter $(k)$, derived from the two-compartment model, needs further assessment. Reflux $k$ is commonly set to zero in DCE-MRI studies that use large-molecular contrast agents $(7,16)$. In our study, $k$ was calculated, but no conclusions were drawn that pertained to differences between treatment groups or differences within a tumor.

Technological advances might optimize DCE-MR: for example, the development of dedicated MR receiver coils (11) that facilitate faster temporal resolutions and/or increase image quality to further improve $K^{P S}$ and $f^{p V}$ measurements. The rationale for seeking further to improve DCE-MRI using clinical MR systems and clinical contrast agents is ultimately to create a functional imaging tool that may be used in standard oncological MRI protocols. DCE-MRI could be of additional value because its functional parameters might objectify the effects of anti-angiogenesis treatment, possibly even before longer time-scale morphological changes, such as reduction in tumor size, become apparent. DCE-MRI might prove a valuable tool for tumor detection, delineation and staging, as well as for differentiating active regions from non-active or necrotic regions within a tumor, and for the optimization of individual anti-tumor treatments. Advances in the areas of MRI contrast agents, pharmacokinetic models, imaging techniques and MRI systems, as well as developments in cancer treatments, are continuing. Our preliminary results suggest that DCE-MRI with a clinical 1.5 Tesla MR system and a widely available. low molecular weight contrast agent may be used to evaluate the effects of angiostatic compounds on tumor angiogenesis in an animal solid tumor model.

A limitation of this study is that the measured inhibition of tumor angiogenesis using DCE-MRI in this melanoma tumor mouse model after treatment with anginex and TNP470 may not predict the effects of other anti-cancer treatments in different tumor models. Nor do our results predict $K^{P S}$ or $f^{p V}$ after treating human tumors with either anginex or TNP-470. Further work will be needed using optimized protocols for human participants to evaluate whether DCE-MRI can indeed successfully demonstrate the effects of antiangiogenesis treatment in patients with solid tumors. 


\section{References}

1. Griffioen $A W_{,}$Molema $G$. Angiogenesis: potentials for pharmacologic intervention in the treatment of cancer, cardiovascular diseases, and chronic inflammation. Pharmacol Rev 2000, 52, 237-268.

2. Folkman J. Angiogenesis in cancer, vascular, theumatoid and other disease. Nat Med 1995, 1. $27-31$.

3. Griffioen AW, van der Schaft DW, Barendsz-Janson AF, ef al. Anginex, a designed peptide that inhibits angiogenesis. Biochem $J 2001,354,233-242$.

4. Dings RP, Yokoyama $Y$, Ramakrishnan $S_{i}$ el al. The designed angiostatic peptide anginex synergistically improves chemotherapy and antiangiogenesis therapy with angiostatin. Cancer Res 2003, 63, 382-385.

5. van der Schaft DW, Dings RP, de Lussanet $Q G_{\text {, t }}$ al. The designer anti-angiogenic peptide anginex targets tumor endothelial cells and inhibits tumor growth in animal models. Faseb $\mathrm{J}$ 2002, 16, 1991-1993.

6. McDonald DM, Choyke PL. Imaging of angiogenesis: from microscope to clinic. Nat Med 2003, 9, $713-725$.

7. Turetschek $K$, Huber $S$, Floyd $E$, et al. MR imaging characterization of microvessels in experimental breast tumors by using a particulate contrast agent with histopathologic correlation. Radiology 2001, 218, 562-569.

8. Padhani AR. Dynamic contrast-enhanced MRI in clinical oncology: Current status and future directions. J Magn Reson Imaging 2002, 16, 407-422.

9. Choyke PL, Dwyer AJ, Knopp MV. Functional tumor imaging with dynamic contrast-enthanced magnetic resonance imaging. J Magn Reson imaging 2003, 17, 509-520.

10. Bhujwalla $Z M$, Artemov $D$, Natarajan $K$, et al. Reduction of vascular and permeable regions in solid tumors detected by macromalecular contrast magnetic resonance imaging after treatment with antiangiogenic agent TNP-470. Clin Cancer Res 2003, 9, 355-362.

11. Kiessling $F$, Heilmann $M_{*}$ Vosseler $S$, ot al. Dynamic $T 1$-weighted monitoring of vascularization in human carcinoma heterotransplants by magnetic resonance imaging. Int $\&$ Cancer 2003, 104, 113-120.

12. de Lussanet $Q G$, Backes $W H$, Griffioen $A W$, et al. Gadopentetate dimeglumine versus ultrasmall superparamagnetic iron oxide for dynamic contrast-enthanced MR imaging of tumor angiogenesis in human colon carcinoma in mice. Radiology $2003,229,429-438$.

13. Yokoyama $Y_{\text {, Dhanabal }} M_{1}$ Griffioen $A W$, ef al. Synergy between angiostatin and endostatin: inhibition of ovarian cancer growth. Cancer Res 2000, 60, 2190-2196.

14. Yamaoka M, Yamamoto $T$, Ikeyama $S$, et al. Angiogenesis inhibitor TNP-470 (AGM-1470) potenily inhibits the tumor growth of hormone-independent human breast and prostate carcinoma cell lines. Cancer Res 1993, 53, 5233-5236.

15. Haacke $M$ "Brown RW. Thompson $M R_{11}$ Venkatesan $R$. $T 1$ estimation from SSI measurements at multiple flip angles. In: Venkatesan $R$, ed. Magnetic resonance imaging: physical principles and sequence design. 1 ed. New: York, NY: John Wiley \& Sons, Inc., 1999, 654-661.

16. Daidrup $H_{0}$ Shames $D M$, Wendland $M$, et al. Correllation of dynamic contrast-enhanced MR imaging with histologic tumor grade: comparison of macromolecular and small-molecular contrast media. Am J Roentgenol 1998, 171, 941-949.

17. Tofts $P S_{i}$ Kermode $A G$. Measurement of the blood-brain barrier permeability and leakage space using dynamic MR imaging. 1. Fundamental concepts. Magn Reson Med 1991, 17, 357-367.

18. Hillen HF, Hak LE, Joosten-Achjanie SR, Arends JW. Micravessel density in unknown primary tumors. Int J Cancer 1997, 74, 81-85.

19. Vermeulen $\mathrm{PB}$, Gasparini $\mathrm{G}$, Fox $\mathrm{SB}$, et al. Second international consensus on the methodology and criteria of evaluation of anglogenesis quantification in solid human tumours. Eur $J$ Cancer $2002,38,1564-1579$.

20. Daldrup HE, Shames DM, Husseini $W$, et al. Quantification of the extraction fraction for gadopentetate across breast cancer capillaries. Magn Reson Med 1998, 40, 537-543.

21. Roberts TP. Turetschelk $K$. Preda $A$, et al. Tumor microvascular changes to anti-angiogenic treatment assessed by MR contrast media of different molecular weights. Acad Radiol 2002, 9 Suppl 2, $\$ 511-513$. 


\title{
Chapter 5
}

\section{Dynamic contrast-enhanced magnetic resonance imaging of radiation therapy induced microcirculation changes in rectal cancer}

\author{
International Journal of Radiation Oncology Biology Physics, 2005: E-pub \\ Quido G. de Lussanet, Walter H. Backes, Arjan W. Griffioen, Anwar R. Padhani, Coen I. Baeten, \\ Angela van Baardwijk, Philippe Lambin, Geerard L. Beets, \\ Jos M. A. wan Engelshoven, Regina G. H. Beets-Tan.
}

\begin{abstract}

\section{PURPOSE}

Dynamic contrast-enhanced T1-weighted magnetic resonance imaging (DCE-MRI) allows non-invasive evaluation of tumor microvasculature characteristics. In this study we evaluated radiation therapy related microvessel changes in locally advanced rectal cancer by DCE-MRI and histology.
\end{abstract}

\section{MATERIALS AND METHODS}

DCE-MRI was performed in 17 patients with primary rectal cancer. Seven patients underwent 25 fractions of 1.8 Gy RT (long-RT) prior to DCE-MRI and ten did not, of whom three patients underwent five fractions of 5 Gy RT (short-RT) in the week before surgery. Endothelial transfer coefficient $\left(K^{\mathrm{PS}}\right)$ measured by DCE-MRI, microvessel density (MVD) (scored by immunoreactivity to CD31 and CD34), and tumor cell- and endothelial cell proliferation (scored by immunoreactivity to Ki67) were compared in the RT treated and non-treated groups.

\section{RESULTS}

Tumor $K^{P S}$ was $77 \%(p=0.03)$ lower in the RT treated group. Histogram analyses showed that RT reduced both magnitude and intra-tumor heterogeneity of $K^{\mathrm{PS}}$ $(p=0.01)$. MVD was significantly lower $(37 \%, p=0.03)$ in tumors that were treated with long-RT than in tumors not treated with RT, but not with short-RT. Endothelial cell proliferation was reduced with short-RT $(81 \%, p=0.02)$ just prior to surgery, but not with long-RT $(p>0.8)$. Tumor cell proliferation was reduced with both long$(57 \%, \mathrm{p}<0.001)$ and short-RT $(52 \%, \mathrm{p}=0.002)$.

\section{CONCLUSION}

DCE-MRI derived $K^{\text {P3 }}$ values show significant radiation therapy related reductions in microvessel blood flow in locally advanced rectal cancer. These findings may be useful for evaluating effects of radiation combination therapies (e.g.. chemoradiation or RT combined with anti-angiogenesis therapy) to account for effects of RT alone. 


\section{Introduction}

Dynamic contrast-enhanced magnetic resonance imaging (DCE-MRI) combined with pharmacokinetic modeling is a promising non-invasive imaging technique for evaluating the tumor microvasculature non-invasively $(1,2)$. While conventionall $M R$ imaging allows qualitative tumor characterization (e.g. size and contrast agent uptake) (3-5), the combination of DCE-MRI with general pharmacokinetic tracer-exchange analysis produce physiological parameters that allow functional characterization of changes in the tumor microvasculature, e.g. tumor angiogenesis (6-8).

In rectal cancer patients, DCE-MRI derived parameters were shown to change early during the course of radiation therapy (RT) (9), and related with the tumor response to chemoradiation combination therapy $(10,11)$. Few studies look at tumor microvessel characteristics after RT alone and none have good histological correlation. In animal tumor models, a DCE-MRI derived parameter for tumor blood flow, the endothelial transfer coefficient $\left(K^{p S}\right)$, was shown to be related with immunohistochemical microvessel density scores (MVD) $(12,13)$ and useful for detecting effects of antivascular treatments (14-16). While RT related antivascular effects are a generally accepted phenomena ( 17 ), the extent by which RT affects the DCE-MRI derived $K^{P S}$ in locally advanced rectal cancer, for example, is currently unknown. As RT is increasingly administered in combination with chemotherapy or experimental therapies, including antivascular agents (18), and DCE-MRI is becoming an accepted non-invasive surrogate marker for evaluating antivascular effects of these combination therapies, possible RT related effects on DCE-MRI derived parameters need be assessed.

The purpose of this study was to evaluate the effects of neoadjuvant RT in patients with locally advanced rectal cancer by DCE-MRI and correlating changes in DCE-MRI with immunohistochemical analyses of tumor vascularization and proliferation of tumor- and endothelial cells.

\section{Patients and Methods}

\section{Patients and treatment}

The ethical review board of our institution approved this study and all patients gave written informed consent. DCE-MRI was performed in 17 patients (median age in years, 63 上 6 [SD]) with curable primary locally advanced rectal cancer. Twelve patients were male and 5 were female ( 3 in the long-RT group, and 2 in the group without RT). Radiation therapy regimens were allocated by the treating clinicians, who had no involvement in the DCE-MRI or histologic data acquisition and analyses. Seven patients underwent a long course of 25 fractions (25 $\times 1.8 \mathrm{~Gy}$, total $45 \mathrm{~Gy}$ ) preoperative RT (long $\mathrm{RT}$ ) prior to DCE-MRI, and 10 patients did not. Three of these 10 patients, kowever, undenwent a short course of 5 fractions ( $5 \times 5 \mathrm{~Gy}$, total $25 \mathrm{~Gy}$ ) preoperative RT (short -RT) in the week before surgery. Because the effect of short-RT is uncertain, these three patients were analyzed as a separate group for the histological analyses, after surgery. Evidently, the time between the start of RT and surgery was significantly ( $p$ $<0.001$ ) longer for long-RT (number of days $\pm \mathrm{SD}_{n} 46 \pm 16$ ) than for short-RT $(8 \pm 2$ ). The average time between MRI and surgery was not significantly different $(p>0.4)$ between the patients with long-RT (number of days $\pm S D, 18 \pm 13$ ), without RT (25 \pm 20 ) or with short-RT $(24 \pm 20)$. 


\section{MR imaging}

DCE-MRI was performed with a conventional clinical MR imaging scanner, at 1.5-Tesla (Intera, Philips, Best, The Netherlands), with a 5-elements cardiac phased array coll. DCE-MRI included 6 pre-contrast T1-weighted measurements $3 \mathrm{D}$ FFE, TR $8.0 \mathrm{~ms}$, TE $3.9 \mathrm{~ms}, 10$ axial slices [slice thickness, $8 \mathrm{~mm}$ ] FOV $290 \times 290 \mathrm{~mm}$, matrix $128 \times 128$, with different flip angles (FAs) $\left(2^{\circ}, 5^{\circ}, 10^{\circ}, 15^{\circ}, 25^{\circ}\right.$, and $\left.35^{\circ}\right)$ to determine the $\mathrm{Tl}$ relaxation time in the blood and tissue before contrast agent arrival on a pixel-by-pixel basis (19). This was followed by the dynamic contrastenhanced series using the same sequence but with a FA of $35^{\circ}$ only (250 scans of 2.4 s) for a total duration of 10 minutes. Gadopentetate dimeglumine (Schering AG, Berlin, Germany; $0.15 \mathrm{mmol} / \mathrm{kg}$ b.w.) was injected intravenously $(3 \mathrm{~mL} / \mathrm{s})$ and flushed with $15 \mathrm{ml}$ saline $(3 \mathrm{~mL} / \mathrm{s})$ at the start of the eight dynamic scan.

\section{MRI data analysis}

MR imaging data were analyzed using a general kinetic two-compartment exchange model as described, for example, by Daldrup et al. $(6,13)$. In short, local T1 relaxation rates $\left(R_{1}\right)$ of the pre-contrast time-averaged images and contrast-enhanced image signal intensity time courses (Figure 1) were used to determine the concentrations of contrast medium in the blood plasma (common femoral arteries), tumor, and gluteus muscles. An experienced $\mathrm{Gl}$ radiologist defined the tumor volume region-of-interest on the T1weighted images. Application of the general kinetic model $(6,13)$ yielded the endothellal transfer coefficient $\left(K^{p S}\right)$, the reflux rate $(k)$, both expressed as $\mathrm{mL}$ per $\min 100$ per $\mathrm{cm}^{3}$ of tissule, and the plasma fraction ( $f^{p v}$ ) values as $\mathrm{mL}$ per $100 \mathrm{~cm}^{3}$ of tissue. Dividing $K^{p s}$ by $k$ yields the volume fraction of the extravascular extracellular space (EES). Values of $K^{P S}, f^{P V}$ and EES in individual voxels were collected for tumor histogram analysis $(20,21)$, and for determining the median tumor values, and median tumor / muscle ratios. Intra-tumor heterogeneity was assessed by the $95^{\text {th }}$ percentile tumor values.

The tumor perfusion index (PI) (mL per min per $100 \mathrm{~cm}^{3}$ of tissue) was also calculated according to the method described by de Vries et al. (11). PI is the maximum of the temporal derivative of the temporal changes in T1-relaxation rate divided by the corresponding T1-relaxation rate change early after contrast injection.

\section{Histology and immunohistochemistry}

Serial sections (thickness, 5um) from the most invasive tumor area were used for immunohistochemical stainings for microvessel density (MVD), and endothelial cell and tumor cell proliferation by double staining of a mixture of Ki-67 antibody (rabbit-antihuman, Clone SP6, Lab Vision, Fremont, CA) and mouse anti-human CD31 (Dako, Glostrup. Denmark) in combination with mouse QBEND-10 anti-CD34 (Monosan Uden, Netherlands). Ki-67 antibody was detected by biotin-labelled swine antimabbit IgG (Dako), incubated with avidine-biotin complex HRP (Dako) and developed with diaminobenzidine (DAB, Sigma). CD31 and CD34 were detected by alkaline phosphatase-labelled goat anti-mouse IgG (Dako). The sections were mounted in imsol (Imsol-Mount, Klinipath, Duiven, The Netherlands).

MVD (CD31/CD34 positive vessels) and endothelial cell proliferation (CD31/CD34 and Ki67 positive) were determined independently by two observers along a predefined line from the tumor luminal side to the deepest invasion, as the total number of vessels divided by the area (number of optic fields of $0.22 \mathrm{~mm}^{2}$ ). The endothelial cell proliferation ratio was calculated by dividing the number of proliferating endothelilal cells by the MVD. The ratio of the number of Ki67 positive tumor cells and total number of tumor cells, in 
the predefined line, were scored as $1(<25 \%), 2(25 \%-75 \%)$, or $3(>75 \%)$. The total number of $K_{i-67}$ positive tumor cells/mm ${ }^{2}$ was counted in three predefined $K \mathrm{Ki}-67$ hot spots (optic field, $0.22 \mathrm{~mm}^{2}$ ).

\section{Statistical methods}

Differences in histologic and immunohistochemical scores, and median DCE-MRI parameters between the groups with and without RT were compared using the twosample (2-tailed) Student t-test. The Pearson correlation coefficient (r) was calculated to reveal a possible relationship between tumor $\mathrm{Pl}$ and $\mathbb{K}^{\mathrm{PS}}$ values. Statistical significance was inferred at the level $p<0,05$.

\section{Results}

\section{DCE-MRI findings}

All patients underwent DCE-MRI without difficulties in the imaging procedures.

A slower 'leakage' of contrast medium into the tumor was found (a difference of $77 \%$ ( $p$ $=0.03)$ ) in patients who received radiotherapy (RT) as was assessed by the endothelial transfer coefficient $K^{P S}$ (Figure 1 and $2 a$, and Table 1).

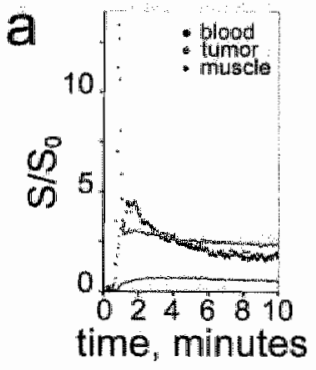

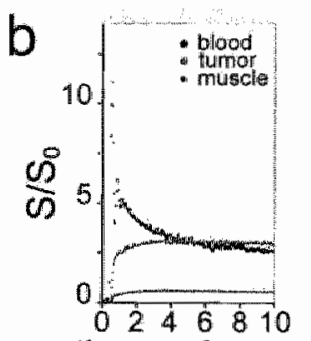

time, minutes
Figure 1. Representative (i.e., median $K^{p s}$ ) signal intensity time courses measured with DCE-MRI in a tumor without RT (a) show that its increase in signal intensity directly after contrast injection (arrow) is smaller but similar to the steep signal increase in the blood including the small re-circulation peak. Conversely, in a tumor with long-RT (b) the signal intensity increase is slower and prolonged (arrow), unlike the blood signal, and the decline in tumor signal is substantially silower than in the tumor without RT 
Histogram analysis of all tumor pixel $K^{P S}$ values illustrated lower ranges in $K^{p S}$ measured in tumors with RT than in tumors without RT (Figure $2 \mathrm{~b}$ ). These lower ranges in tumor $K^{P S}$ values in tumors with RT were reflected by the lower $95^{\text {th }}$ percentile tumor $K^{\text {Ps }}$ values $(p=0.02)$, and in terms of the tumor / muscle ratio $(p=0.03$ ) (Table 1$)$. In contradistinction, $K^{P S}$ in muscle tissue was slightly higher in tumors with RT than tumors without $R T$ (n.s., $p>0.3$ ) (median $\pm S D, 8.1 \pm 8.1$ after $R T$, and $4.6 \pm 2.8$ without $R T$ ).

a

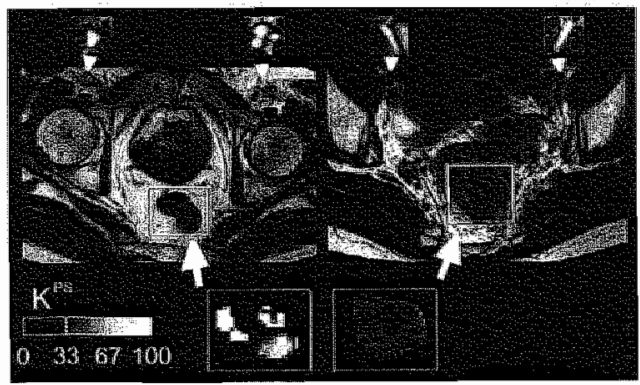

$\mathrm{K}^{\mathrm{PS}}$ map

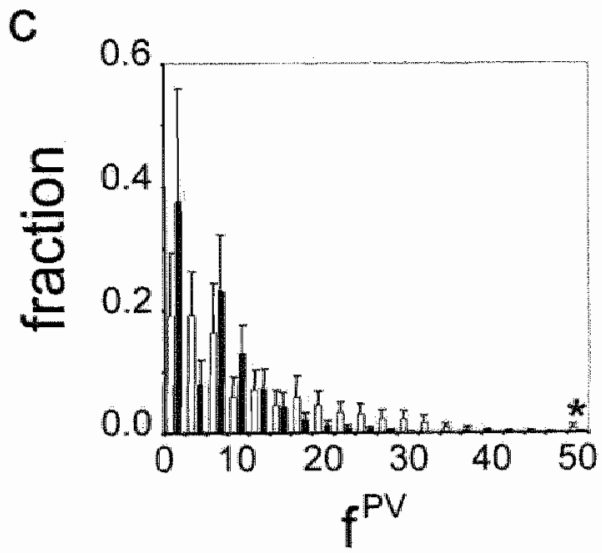

b
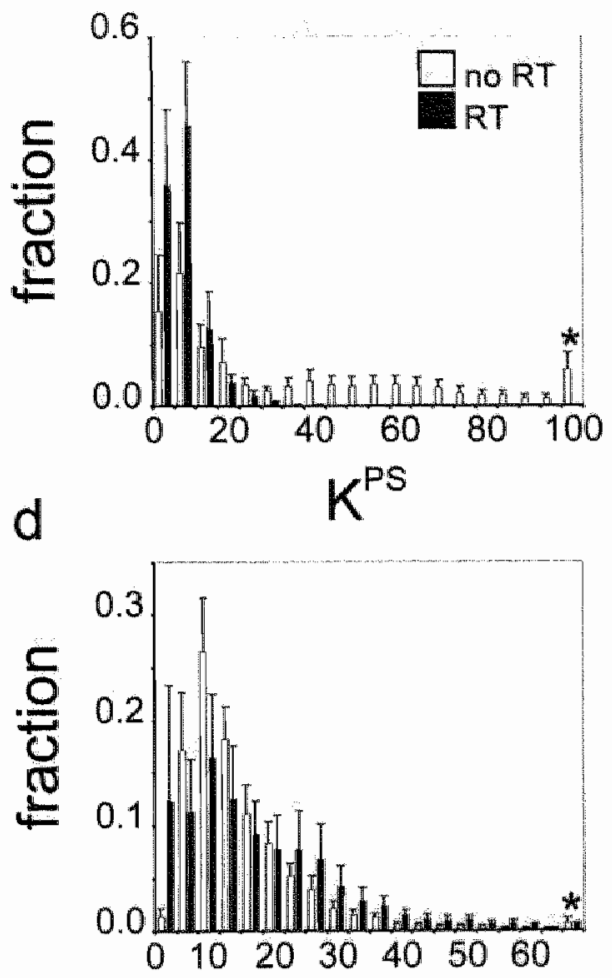

EES

Figure 2. (a) Axial T2-weighted images were used for locating the tumor, shown here are tumors with the median $K^{p S}$ without RT (left) and with long-RT (right); the top inserts illustrate the signal enhancement in the common femoral arteries directly after contrast injection on the corresponding $T 1$-weighted acquisition; the bottom inserts are the grayscale ${ }^{\text {m*t }}$-coded tumor $K^{p s}$ maps, which illustrate that $K^{p S}$ is lower in magnitude and heterogeneity after long-RT. Averaged histogram distribution plots for all tumors show that (b) $K^{\mathrm{PS}}$ and (c) $f^{\mathrm{PV}}$ are lower and less heterogeneous (i.e. inter-tumor variation) in tumors with long-RT (black bars) than in tumors without RT (white bars); while (d) EES is just slightly increased in tumors with long-RT. The error bars (SEM) illustrate the inter-tumor variation. "Indicates that the highest values $\left(K^{\mathrm{PS}}>95.0\right.$, EES $>63.3$, and $\left.f^{\mathrm{PV}}>47.5\right)$ were pooled. "Color image is on the back-cover. 
Table 1 DCEMRI derived parameters

\begin{tabular}{|c|c|c|c|c|}
\hline & & & Hes & tumor muscle ratio \\
\hline & & medilan & 95th percentile & \\
\hline$K^{+9 s}$ & $\begin{array}{r}\text { no RT } \\
\text { Ionig-RT }\end{array}$ & $\begin{array}{c}28.6 \pm 26.8 \\
6.5 \pm 2.4\end{array}$ & $\begin{array}{l}67.8 \pm 55.2 \\
15.4 \pm 4.1^{\text {.x }}\end{array}$ & $\begin{array}{l}6.5 \pm 6.3 \\
1.5 \pm 1.0^{\circ}\end{array}$ \\
\hline$r^{P V}$ & $\begin{array}{r}\text { no RT } \\
\text { long-RT }\end{array}$ & $\begin{array}{l}2.3 \pm 2.0 \\
1.4 \pm 1.1\end{array}$ & $\begin{array}{l}1.8 \pm 1.7 \\
1.2 \pm 0.9\end{array}$ & - \\
\hline EES & $\begin{array}{c}\text { no } \mathbb{R T} \\
\text { long-RT }\end{array}$ & $\begin{array}{l}0.39 \pm 0.15 \\
0.43 \pm 0.11\end{array}$ & $\begin{array}{l}0.31 \pm 0.15 \\
0.35 \pm 0.13\end{array}$ & $\begin{array}{l}1.3 \pm 0.3 \\
2.1 \pm 1.3\end{array}$ \\
\hline Note & $\begin{array}{l}\text { Both the erd } \\
\text { magnitude a } \\
\text { extravascular } \\
\text { Walues preser } \\
\text { 1: SD, which is } \\
\text { The no RT g } \\
\text { MRl) and the } \\
\text { "indicates sig }\end{array}$ & $\begin{array}{l}\text { lial transfer co } \\
\text { variation after } \\
\text { racelituar space } \\
\text { are the media } \\
\text { standard devi } \\
\text { had } n=10 \mathrm{~F} \\
\text { gT group } n= \\
\text { cant difference }\end{array}$ & $\begin{array}{l}\left(K^{P}\right) \text { and blood pla } \\
\text { therapy (long-RT } \\
\text { ncreases slighty. } \\
\text { vidual tumor vallues } \\
\text { d represents the inte } \\
7 \text { without RT and } \\
\text { lo. } \\
\text { long-RT and no RT }\end{array}$ & 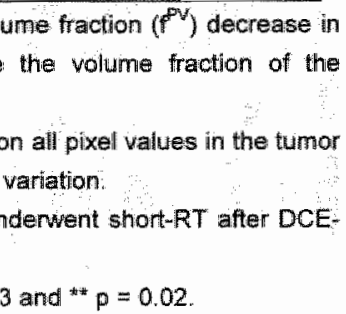 \\
\hline
\end{tabular}

A significant correlation $(r=0.74, p<0.001)$ that was observed between the tumor $K^{\text {PS }}$ and the perfusion index (PI) for all tumors combined "reflects the gross similarity between these parameters. Unlike $K^{\text {PS }}$, however, $\mathrm{Pl}$ did not indicate significant differences in relation to $\mathrm{RT}(\mathrm{p}=0.5)$ (median $\mathrm{Pl} \pm \mathrm{SD}$ was $34 \pm 10$ after $\mathrm{RT}$, and $39 \pm$ 21 without $(R T)$.

Histogram analysis suggested lower $f^{p v}$ values in tumors with RT than tumors without RT (Figure 2c), however, these differences were not significant, neither for median tumor $f^{p}$ values $(p=0.3)$ nor for $95^{\text {th }}$ percentile $(p=0.3)$ values (Table 1). Because of the fact that $f^{P V}$ values were very low (sometimes approaching zero), the ratio of tumor / muscle $f^{p V}$ did not result in realistic values and were therefore not calculated.

No significant $(p>0.4$ ) changes were observed in tumor EES values. Tumor / muscle ratios were about twice as higlh (n.s., $p>0.1)$ in tumors with $R T$ than in tumors without RT, however ((Table 1, Figure 2d).

Histological findings

Tumor cell proliferation was significantly lower $(p=0.0006)$ in the tumors that were treated with long-RT than in the tumors that were not treated with $R T$, also in the three tumors with short RT lower tumor cell proliferation was observed $(p=0.02)$ (Figure 3a).

The long-RT regimen resulted in significantly $(p=0.03)$ tower MVD scores, as compared to the non-irradiated tumors. (Figure $3 b$ ), but the short-RT regimen did not. Endothelial cell proliferation ratios were significantly $(p<0.05)$ lower after the short-RT regimen that was administered during the week before surgery, as compared to non-irradiated tumors, but not after long-RT $(p>0.8)$ that was administered several weeks before surgery, (Figure 3c). 

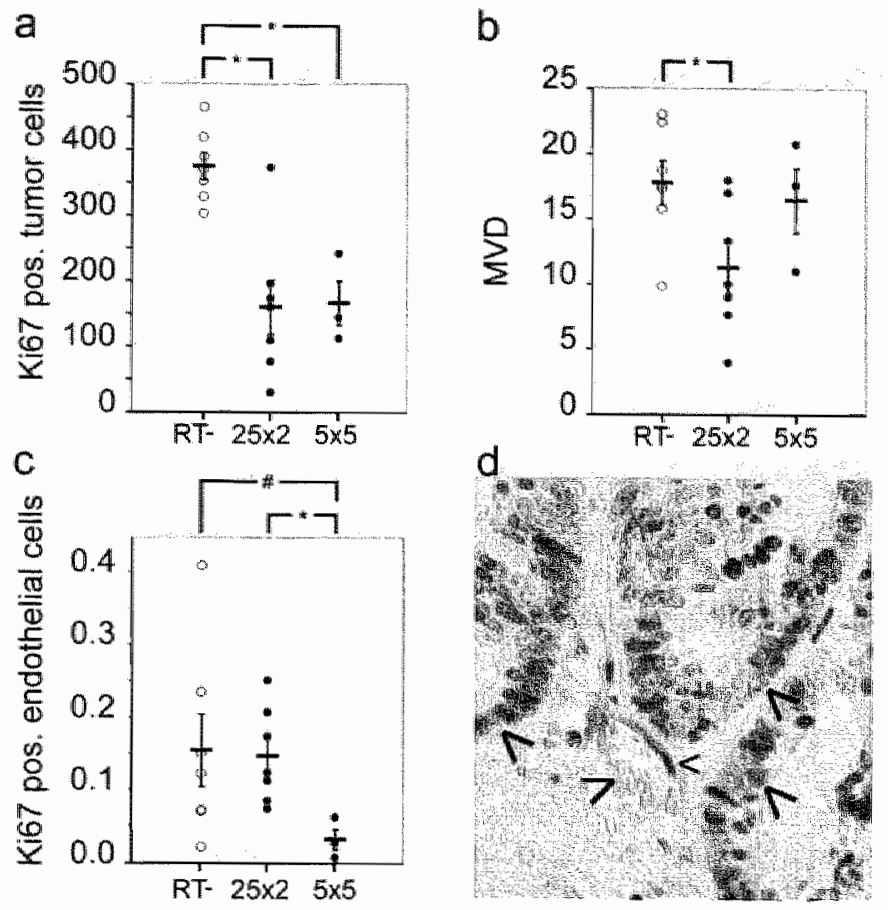

Figure 3. (a) The average number of Ki-67 positive tumor cells was significantly (") higher in tumors without RT (RT-) than in tumors with long-RT and short-RT: (b) microvessel density (MVD) was significantly (") lower only after long-RT (with respect to RT-); (c) Ki-67 endothelial cell proliferation ratio was lower only after short-RT; (d) tumor cell proliferation is marked by the brown* nuclear staining of tumor cells (to Ki67) (large diagonal arrows), the arrowheads depict tumor blood vessels (blue**, staining of endothelial cells to CD3f and CD34); note the brown * staining of the typical elongated nucleus in an endothelial cell (blue ${ }^{* *}$ ) suggesting angiogenic endothelial cell proliferation in the tumor without RT (small horizontal arrow). Significant differences are indicated with * $(p<0.02$ ) and $(p<0.05)$. The magnification in $d$ is $400 x$ ** Color image is an back cover (right).

\section{Discussion}

The results of this study demonstrate significant radiation therapy related microcirculation changes in locally advanced rectal cancer by DCE-MRI and histology. The DCE-MIRI derived endothelial transfer coefficient, $K^{P S}$, measured using a low molecular weight (MW) contrast agent largely reflects tumor blood flow, as well as the leakiness (i.e. microvessel permeability surface area product) of the tumor microvessels $(21,22)$. Therefore, $K^{\text {PS }}$ was effective in showing effects of long-RT that occur approximately $2-4$ weeks after the initiation of $R T(17)$.

RT damages all blood vessels, but specific effects depend on vessel size "location, and dose-time-volume factors (17). While acute effects of RT are marked by increased microvessel permeability, related to endothelial cell damage and local inflammation. longer term effects are marked by decreased permeability resulting from basement membrane thickening and extracapillary fibrosis (17). Additional chronic effects of RT 
are the inhibition of capillary sprouting and wascular remodeling (23), reduced MVD, endothelial cell destruction, and reduced microvessel functionality by mural thrombi and obliteration of the vessel lumen (17).

$K^{\text {Pis }}$ is expected to be a more successful marker than other DCE-MRll derived markers such as perfusion index (PI) and area under the signal-intensity-time curve (AUC), because $K^{\text {Prs }}$ represents a single microcirculatory parameter, whereas $P$ I is a perfusion parameter that depends not only on $K^{\text {PS }}$ but is also confounded by (non-significant) changes in $f^{p V}$ and EES (22). For example, our study shows a significant $77 \%$ reduction in $K^{P S}$ while PI was only $15 \%$ (and non significantly) reduced, despite the strong pasitive relationship between $\mathrm{PI}$ and $K^{\mathrm{P} S}$. De Vries et al. (9) reported increases in $P \mid$ during the first weeks of RT in rectal cancer, which corresponds very well with reported acute effects of RT (17). The reported increases in PI were $25 \%$ at most, and the observed possible decline in PI after the final (fourth) week of RT was not significant (9).

Presenting the true tumor $K^{P S}$ may be preferable over the tumor / muscle $K^{P S}$ ratio, because effects of RT in muscle are uncertain. The gluteus muscles, for example, is several centimeters posterior to the rectal tumor, but is still at least partially within anterior-posterior field of radiation. Muscle is generally assumed to demonstrate minimal response to clinical irradiation, however, transient alterations as well as varying degrees of atrophy and necrosis have been reported for skeletal muscle following single doses of $\mathrm{RT}$ (24). High dose radical RT can cause persistent muscle edema, often visible on MRI, probably reflecting long-term damage of muscle microcirculation. The increase in muscle $K^{P S}$ observed in the current study did not affect the observed statistical differences in tumor $K^{p S}$ in relation to therapy when expressed as a tumor / muscle ratio.

The DCE-MRI derived volume fraction of the extravascular extracellular space, EES, showed some increased intra-tumor variation after RT. Cell destruction caused by radiation might have increased the relative extracellular volume to which the contrast agent might leak, hence increasing EES. While in other parts of the tumor, infiltration of inflammatory cells and tissue fibrosis (25) might have decreased EES.

Immunohistochemical results reflected anti-tumor and anti-angiogenesis effects of RT. Direct measures of anti-angiogenesis effects are reduced MVD and reduced Ki67 immunoreactivity of endothelial cells. Our data shows incongruous effects between longand short $\mathrm{RT}$, although it is noted that the number of patients that underwent short-RT was limited. Explanations why short-RT showed low endothelial cell proliferation without differences in MVD, and long-RT showed low MVD without differences in endothelial cell proliferation, as compared to tumors without $R T$, might be related to the higher fraction dosage with short-RT and / or the shorter time interval between RT and surgery with short-RT, than with long-RT. It may be possible that despite lower fraction dosages, long-RT had short term effects on endothelial cell proliferation, but that endothelial cell proliferation recovered during the time interval between RT and surgery, especially because the fraction dosages were lower (26). Since, the time between the completion of RT and surgery was less than a week for short-RT and several weeks for long-RT, it is also presumable that in short-RT we were observing the acute effects such as endothelial cell damage with reduced proliferation, and in long-RT we were observing the long term effects including endothelial cell destruction and MVD reduction (17). A larger number of patients would have allowed for the evaluation of possible relationships between, for example, $K^{P S}$ and MVD as demonstrated in animal studies $(12,13)$. Patient inclusion was stopped, because of changes in treatment protocols at our hospital $(27,28)$. Accurate quantification of $\mathrm{K}^{\mathrm{PS}}$ was hindered by the large inter- and intra-tumor 
heterogeneity, particularly in the non-irradiated patient group, and limited size of the patient groups. Future studies should therefore use larger sample sizes for comparing patient groups with different treatment regimes (e.g short-versus long-RT). Similarly, co-localizing MR imaging and histological parametters might considered more desirable than our use of one "representative" (e.g., median) value for each parameter. For example, co-localization between $K^{\mathrm{PS}}$ and MVD was demonstrated in animal tumor models, in which the in-plane resolution of MRI $(0.5 \times 0.5 \mathrm{~mm})$ matched the optic field of view the histologic sections, histologic sections could be closely matched with the MRI sections, and maximum tumor diameters were typically less than $1 \mathrm{~cm}(14)$. However, one might imagine the sampling errors that may occur by attempting to co-localize MRI and histology in these large rectal tumors in patients, and the spatial resolution on MRI was substantially lower than were obtained in mice.

MVD is commonly used as surrogate marker for the tumor microvascullature to validate DCE-MRI derived parameters. However, one important note is that MVD may not fully equate to the DCE-MRI parameters, as MVD is dependent on the staining of endothelial cells in blood vessels and is thus a morphologic marker, while DCE-MRI derived parameters depend on microcirculatory perfusion dependent and are therefore functional markers.

The anti-tumor effect of RT was most evident in the reduction of proliferating tumor cells as detected by Ki67 immunoreactivity in tumor cells, observed after both long-and shortRT regimens, consistent with earlier reports (29). Tumor cell immunoreactivity for Ki67 is not a direct measure for angiogenesis in rectal cancer, however, it might be postulated that RT related reductions in tumor cell proliferation reduces their production of proangiogenic factors, and thus lead to an angiostatic state.

\section{Clinical implications}

While, conventional gadolinium-enhanced MR sequences have limited additional value for morphologic diagnostic assessment of rectal cancer (5), new gadolinium-enhanced MR protocols, such as DCE-MRI, will complement conventional imaging of tissue morphology by non-invasive evaluation of (tumor) tissue physiology. DCE-MRI was used here to show that radiation therapy alone significantly inhibits angiogenesis in rectal cancer. Developments in anti-angiogenesis therapy are improving the prospects for anticancer treatment $(30,31)$, including disseminated colorectal disease $(32,33)$, when combined with neoadjuvant radiation therapy $(18,34)$, or chemotherapy $(35,36)$. This study provides a reference for future evaluations of additive effects of anti-angiogenesis treatments that are supplemented to neoadjuvant radiation therapy in rectal cancer. 


\section{References}

1. MeDonald DM, Choyke PL. Imaging of anigiogenesis: from microscops to clinic. Nal Med 2003,9713-725.

2. Leach MO, Brindle $K M$, Evelhochi $J$ et al. Assessment of antiangiogenic and antivascular therapeutics using MRI: recommendations for appropriate methodology for clinical trials. $\mathrm{Br} J$ Radiol 2003; 76 Spec No 1:S87-91.

3. Beets Tan $\mathrm{RO}$, Beets $\mathrm{GL}$, Vliegen $\mathrm{RF}$ el al. Accuracy of magnetic resonance imaging in prediction of tumour-free resection margin in rectal cancer surgery. Lancet 2001;357:497-504.

4. Beets-Tan RG, Beets GL. Rectal cancer: review with emphasis an MR imaging: Radiology 2004:232:335-346.

5. Vliegen RF, Beets GL, von Meyenfeldt MF ef al. Rectal Cancer: MR Imaging in Local Staging -Is Gadolinium-based Contrast Material Helpful? Radiology 2005;234:179-188.

6. Daldrup HE, Shames DM, Husseini W, Wendland MF, Okuhata $Y$, Brasch RC. Quantification of the extraction fraction for gadopentetate across breast cancer capillaries. Magn Reson Med $1998: 40: 537-543$.

7. Padhani AR. Functional MRI for anticancer therapy assessment. Eur J Cancer $2002 ; 38: 2116$ 2127.

8. Knopp $M V$, Von Tengg-Kobligk $H$, Choyke PL. Functional magnetic resonance imaging in oncology for diagnosis and therapy monitoring. Mol Cancer Ther 2003;2:419-426.

9. de Wries $\mathrm{A}_{\text {s }}$ Griebel J, Kremser $\mathrm{C}$ et al. Monitoring of tumor microcirculation during fractionated radiation therapy in patients with rectal carcinoma: preliminary results and implications for therapy. Radiology 2000;217:385-391.

10. George ML, Dzik-Jurasz AS, Padhani AR et all. Non-invasive methods of assessing angiogenesis and their value in predicting response to treatment in colorectal cancer. $\mathrm{Br} J \mathrm{Jurg} 2001 ; 88: 1628-$ 1636.

11. Devries $A F$, Griebel $\mathrm{J}$, Kremser $\mathrm{C}$ et al. Tumor microcirculation evaluated by dynamic magnetic resonance imaging predicts therapy autcome for primary rectal carcinoma. Cancer Res $2001 ; 61: 2513-2516$.

12. van Dijke $C F$, Brasch RC, Roberts TP et al. Mammary carcinoma model: correlation of macromolecular contrast- enhanced MR imaging characterizations of tumor microvasculature and histologic capillary density. Radiology 1996;198:813-818.

13. de Lussanet $Q G$, Backes $W H$. Griffioen $A W$, van Engelshoven JM, Beets-Tan RG. Gaclopentetate dimeglumine versus ultrasmall superparamagnetic iron oxide for dynamic contrast-enhanced MR imaging of tumor angiogenesis in human colon carcinoma in mice. Radiology 2003;229:429-438.

14. de Lussanet $Q G$, Beets-Tan $R G$, Backes WH et all. Dynamic contrast-enhanced magnetic resonance imaging at 1.5 Tosla with gadopentetate dimeglumine to assess the angiostatic effects of anginex in mice. Eur $J$ Cancer 2004:40:1262-1268.

15. Bhujwalla ZM. Artemov D, Natarajan $K$, Solaiyappan M, Kollars $P$, Kristjansen PE. Reduction of vascular and permeable regions in solid tumors detected by macromolecular contrast magnetic resonance imaging after treatment with antiangiogenic agent TNP-470. Clin Cancer Res 2003;9:355-362.

16. Evelhoch JL, LoRusso PM, He $Z$ at al. Magnetic resonence imaging measurements of the response of murine and human tumors to the vascular-targeting agent ZD6126. Clin Cancer Res $2004 ; 10: 3650-3657$.

17. Byhardt RW, Moss WT. The Heart and Blood Vessels. In: Cox JD, ed. Moss' Radiation Oncology. 7 ad. St. Louls: Mosby, 1994:311-319.

18. Wachsberger $P$, Burd $R$, Dicker AP. Tumor response to lonizing radiation combined with antiangiogenesis or vascular targeting agents: exploring mechanisms of interaction. Clin Cancer Res 2003:9:1957-1971.

19. Haacke M, Brown RW. Thompson MR, Venkatesan R. T4 estimation from SSI measurements at multiple flip angles. In: Haacke $M$, Brown RW, Thompson MR, Venkatesan $R$, eds. Magnetic resonance imaging: physical principles and sequence design. 1 ed. New York, NY: John Wiley \& Sons, tnc., 1999:654-661.

20. Hayes $C$, Padhani AR, Leach MO. Assessing changes in tumour vascular function using dynamic contrast-enhanced magnetic resonance imaging. NMR Biomed 2002;15:154-163. 
21. de Lussanet QG Langereis S, Beets-Tan RG et al. Relationship Between Dynemic ContrastEnhanced MR Imaging Kinetic Parameters and Molecular Weight of Dendrite Contrast Agents in Tumor Angiogenesis in Mice. Radiology 2004, in press.

22. Tofts $P S$, Brix $G$, Buckley $D$ L al. Estimating kinetic parameters rom dynamic contrastenhanced T(1)- weighted MRl of a diffusable tracer: standardized quantities and symbols. J Magn Resion Imaging 1999:10:223-232.

23. Van Den Brenk HA. The effect of lonizing radiations on capillary sprouting and vascular remodelling in the regenerating repair blastema observed in the rabbit ear chamber. Am $J$ Roentgenal Radium Ther Nucl Med 1959;81:859-884:

24. Lewis RB. Changes in striated muscle following single intense doses of $x$-rays. Lab Invest $1954 ; 3: 48-55$.

25. Dworak $O$, Keilholz $L$. Hoffmann A. Pathological features of rectal cancer after preoperative radiochemotherapy. Int J Colorectal Dis 1997;12:19-23.

26. Koukourakis MI. Giatromanolaki A, Sivridis $E$ et al. Squamous cell head and neck cancer: evidence of angiogenic regeneration during radiotherapy. Anticancer Res 2001:21:4301 4309.

27. Adjuwant radiotherapy for rectal cancer: a systematic overview of 8,507 patients from 22 randomised trials. Lancet $2001 ; 358: 1291-1304$.

28. Kapiteijn E, Marijnen CA, Nagtegaal ID et al. Preoperative radiotherapy combined with total mesorectal excision for resectable rectal cancer. N Engl J Med 2001;345;638-646.

29. Adell $G$, Zhang $H_{1}$ Jansson $A$, Sun $X F$, Stal $O$, Nordenskjold $B$. Decreased tumor cell proliferation as an indicator of the effect of preoperative radiotherapy of rectal cancer. Int $\mathrm{J}$ Radiat Oncol Biol Phys 2001:50:659-663.

30. Carmeliet $P$, Jain RK. Angiogenesis in cancer and other diseases. Nature 2000;407:249-257.

31. Griffioen AW, Molema $G$. Angiogenesis: potentials for pharmacologic intervention in the treatment of cancer, cardiovascular diseases, and chronic inflammation. Pharmacol Rev 2000;52:237-268.

32. Willett CG, Boucher $Y$, di Tomaso $E$ of al. Direct evidence that the VEGF-specific antibody bevacizumab has antivascullar effects in human rectal cancer. Nat Med 2004, 10:145-147.

33. Morgan $B$, Thomas AL, Drevs $J$ et at. Dynamic contrast-enhanced magnetic resonance imaging as a biomarker for the pharmacological response of PTK787/ZK 222584, an inhibitor of the vascular endothelial growth factor receptor tyrosine kinases, in patients with advanced colorectal cancer and liver metastases: results from two phase I studies. J Clin Oncoll 2003:21:3955-3964.

34. Dings RP, Williams BW, Song CW, Griffioen AW, Mayo KH, Griffin RJ Anginex synergizes with radiation therapy to inhibit tumar growth by radiosensitizing endothelial cells. Cancer Research 2004;in press.

35. Teicher $B A$, Sotomayor $E A$, Huang $Z D$. Antiangiogenic agents potentiate cytotoxic cancer therapies against primary and metastatic disease. Cancer Res 1992;52:6702-6704.

36. Jain RK. Normalizing tumor vasculature with anti-anglogenic therapy: a new paradigm for combination therapy. Nat Med 2001;7:987-989. 


\section{Chapter 6}

\section{Dynamic contrast-enhanced MR imaging of perfusion recovery and MR angiography of collateral growth in a femoral artery ligation model}

Submitted, 2005

Quido G. de Lussanet, Jalanda C.G. van Golde, Regina G.H. Beets-Tan, Mark J. Post, Maya S. Huijberts, Nicolaas C. Schaper, Alphons G.H. Kessels, Jos M.A. van Engelshoven, Walter H. Backes.

\section{ABSTRACT}

\section{PURPOSE}

Serial MR imaging was performed to monitor collateral vessel growth and muscle blood flow recovery in a peripheral ischemia model.

\section{MATERIALS AND METHODS}

Dynamic contrast-enhanced MR imaging (DCE-MRI) and contrast-enhanced MR angiography (MRA) were performed, with institutional review board approval, in 15 male rabbits, 2 hours (day $0^{+}$), 7 days, and 21 days after femoral artery ligation. DCE-MRI monitored resting muscle blood flow in terms of endothelial transfer coefficient $\left(K^{\mathrm{Ps}}\right)$ in the anterior tibial and soleus muscle. High spatial resolution MRA visualized submillimeter collaterall arteries.

\section{RESULTS}

$\mathrm{K}^{\mathrm{PS}}$ in the anterior tibial muscle of the ligated limb was $23 \%$ of the $\mathrm{K}^{\mathrm{PS}}$ in the control limb, on day $0^{+}, 81 \%$ on day 7 and $85 \%$ on day $21 . K^{P S}$ in the soleus muscle of the liglated limb was $63 \%$ on day $0^{*}, 85 \%$ on day 7 and $90 \%$ on day 21 . The number of collaterals around the liglated femoral artery was 1.1 on day $0^{+}, 4.2$ on day 7 , and 6.0 on day 21 .

\section{CONCLUSION}

MRI allows non-invasive, serial monitoring of changes in muscle blood flow, and submillimeter collateral vessel growth, in a rabbit femoral artery ligation model. 


\section{Introduction}

Investigations of arterial occlusive disease and disease models have lead to important developments in therapeutic neo-vascularization ${ }^{1 / 3}$. Therapeutic strategies for neovascularization may involve one or a combination of the following (i) stimulation of collateral vessel growth (i. $e_{n}$, arteriogenesis) ${ }^{4}$ to restore bllood flow to ischemic tissues ${ }^{5-}$ ${ }^{7}$ and (ii) stimulation of capilary sprouting (i,e., angiogenesis) ${ }^{4}$ with vessel remodeling (i.e., angioadaptation) ${ }^{8}$ to restore tissue perfusion ${ }^{9-11}$. A challenge in therapeutic neovascularization is to create surrogate markers that allow non-invasive evaluation of angiogenesis and arteriogenesis stimulating treatments.

One potential surrogate marker for neo-vascularization is dynamic contrast-enhanced MR imaging (DCE-MRI) ${ }^{12.13}$. The potential of DCE-MRI for evaluating neovascularization was established in oncology in numerous studies performed over the past decade ${ }^{14-17}$. Some of the demonstrated uses of DCE-MRI were to monitor effect of treatment, predict treatment outcome, and differentiate between benign and malignant disease. In addition, the reproducibility of DCE-MRI in skeletal muscle was established 18

DCE-MRI is performed by combining dynamic MR imaging data with a general pharmacokinetic model, to quantify specific microcirculatory parameters. One effective microcirculatory parameter is the endothelial transfer coefficient $\left(K^{P S}\right.$, or its equivalent $k^{\text {trains }}$ ). $K^{\mathrm{PS}}$ reflects a combination of either microcirculatory flow, or microvessel permeability and surface area ${ }^{19}$, depending on the molecular weight of the MR contrast agent used. DCE-MRI is useful for evaluating microvessel permeability (-surface area) when a high molecular weight MR contrast agent is used, and alternatively useful for evaluating microcirculatory flow when a low molecular weight MR contrast agent is used. Recently. DCE-MRI was shown ${ }^{20}$ to be effective for monitoring angiogenesis in an ischemic injury model, by recording the angiogenesis related changes in microvessel permeability (-surface area), as measured with use of a high molecular weight MR contrast agent.

It is hypothesized here that DCE-MRI with use of a low molecular weight MR contrast agent, is effective for monitoring arteriogenesis following femoral artery ligation, by quantifying changes in blood flow in the lower hindlimb muscles as collateral vessels develop. The development of collateral vessels can be established non-invasively by serial MR angiography (MRA) ${ }^{21-26}$.

This study evaluates the use of MRI for serial evaluations of neo-vascularization, specifically arteriogenesis, in a rabbit femoral artery ligation model. To this end, a combined MR imaging protocol was designed in which both MRA and DCE-MRI were implemented to (I) monitor collateral vessel growth and (II) quantitate the respective changes in muscle blood flow.

\section{Materials and Methods}

\section{Animal model}

To evaluate MR imaging of perfusion recovery, a validated femoral artery ligation rabbit model was adopted ${ }^{7}$. The study was approved by the institutional ethicall review board for animal studies, accredited by the National Department of Health. Fifteen rabbits (New Zealand White) (weight range, $2.8-3.5 \mathrm{~kg}$ ) were included. During the experiments the rabbits were ventilated with isoflurane $(2-3 \%)$. The left femoral artery was ligated (day 0 ), 
under sterile conditions, by placing 2 ligations $(1-2 \mathrm{~cm}$ apart) distal to the branches of the lateral circumflex-and deep arteries ${ }^{\bar{T}}$. A sham operation was performed in the right hindlimb (i.e. non-ligated limb). The measurements in the non-ligated limb were used as control measurements. No pressure sores or signs of gangrene were observed in the ligated limbs. Post-operative analgesia was administered once a day "for 3 days, by intramuscular injection of buprenorphine. Directly after the final MR measurement at day 21 a lethal dose of pentobarbital ( $1 \mathrm{ml} / \mathrm{kg}$ body weight) was injected intravenously. The serial study design, rather than a cohort study resulted in relatively high drop-out rate. Three rabbits had died during the first week (day $7, n=12$ ) and three more rabbits had died during the second and third week (day $21, n=9$ ). In addition, one of the fifteen animals was not imaged on day $0^{*}$ owing to technical problems.

MR imaging

DCE-MRI was performed 2 hours (day $\left.0^{+}\right), 7$ days, and 21 days after femoral artery ligation, using a 1.5 Tesla MRI system (Philips Intera Philips Medical Systems, Best $_{i}$ The Netherlands) with a maximum gradient strength of $30 \mathrm{mT} / \mathrm{m}$ and rise time of $200 \mu \mathrm{s}$. Rabbits were imaged in supine position using a 5-elements cardiac phased array coil. The advantage of using this coil is that both limbs could be imaged in full without repositioning the rabbit. To both the DCE-MRI and MRA acquisitions, spatial homogeneity corrections (Constant Level AppeaRance (CLEAR), Philips Medical Systems) were applied based on the spatial sensitivity information derived from a reference scan, which was performed first, using the quadrature body coil.

The DCE-MRI protocol included a pre-contrast measurement (3-dimensional fast fieldecho (3D FFE), echo time / repetition time (TE/TR) 2.2/4.5 msec (acquired) pixel size $2.0 \times 1.65 \mathrm{~mm}, 10$ coronal $5 \mathrm{~mm}$ sections (interpolated during reconstruction to 202.5 $\mathrm{mm}$ thick sections), fieldi-of-view (FOV) $256 \times 192 \mathrm{~mm}$, acquired matrix 128×123) with 6 different flip angles (FA) $\left(2^{\circ}, 5^{\circ}, 10^{\circ}, 15^{\circ}, 25^{\circ}\right.$ and $\left.35^{\circ}\right)$ to determine the pre-contrast $T_{1}$ relaxation times before contrast enhancement ${ }^{26}$, and a $T_{1}$-weighted dynamic contrastenhanced series with the same imaging parameters (FA $35^{\circ}$ ) and 200 dynamic volume acquisitions, duration $10 \mathrm{~min}^{27}$. Gadopentetate dimeglumine $(0.5 \mathrm{mmol} \mathrm{Gd} / \mathrm{mL}$; dosage, $0.2 \mathrm{~mL} / \mathrm{kg}$ ) (Magnevist, Schering, Berlin, Germany) (molecular weight, $0.5 \mathrm{kDa}$ ) was injected manually into an ear vein ( $15 \mathrm{~s}$ injection duration) at the start of the $8^{\text {th }}$ dynamic volume acquisition and then flushed with $2 \mathrm{~mL}$ saline.

MRA was performed 10 minutes after DCE-MRI (i.e., 20 minutes after the first MR contrast agent injection). The MRA protocol ${ }^{21}$ consisted of a pre-contrast acquisition (mask) and a contrast enhanced acquisition, with the same imaging parameters, T1weighted acquisition (3D FFE; TE/TR 2.6/9.1 msec; FA 30 , acquired voxel size $0.6 \times 0.6 \times 0.7 \mathrm{~mm}, 80$ coronal sections, FOV $196 \times 176 \mathrm{~mm}$, acquired matrix $320 \times 320$, duration 150 seconds per volume acquisition). Eight seconds before starting the contrast-enhanced MRA acquisition gadopentetate dimeglumine $(0.2 \mathrm{~mL} / \mathrm{kg}$ body weight) was manually injected intravenously ( $30 \mathrm{~s}$ injection duration) and then flushed with $2 \mathrm{~mL}$ saline. The total duration of the combined MR imaging protocol (DCE-MRI and MRA) was less than 30 minutes.

\section{Image analysis}

DCE-MRI images were analyzed using a general pharmacokinetic model ${ }^{27}$. In short local $T 1$ relaxation rates $\left(R_{1}\right)$ of the seven pre-contrast time-averaged volume acquisitions and the contrast-enhanced image signal intensity time courses were used to determine the concentrations of the contrast agent in the blood plasma and in muscle 
tissue. For the blood plasma measurements, the voxel with the strongest signal enhancement directly after contrast agent injection. was sellected in the area just proximal to the aorta bifurcation, where the least partial volume effects are to be expected, and were found to give better fits than averaging multiple voxels within the aorta (results from a pillat study, data not shown). For the signal intensity measurements in muscle, three-dimensional regions of interest were defined in the anterior tibial- and soleus muscle of the lower hindlimb, for both the ligated-and non-ligated limb. Typically the regions of interest encompassed 4 or 5 sections. The three-dimensional muscle regions of interest were at least one pixel away from arteries and later enhancing veins, which could be identified on the images of the dynamic contrast enhanced series. Application of the pharmacokinetic model ${ }^{27,28}$ yielded the endothelial transfer coefficient $\left(K^{p s}\right)^{27}$ for each voxel, which was previously shown to reflect microcirculatory flow when using a low molecular weight (i.e., extravascular) MR contrast agent ${ }^{19}$.

Contrast agent arrival times in the aorta- and femoral artery bifurcation were obtained from the DCE-MRI data to obtain the respective arterial inflow delay times, for the ligated and non-ligated limb.

For quantification of collateral growth on the MRA acquisitions, arterial collateral vessels were counted by consensus by two observers using the Longland definition ${ }^{29}$. In short, the Longland definition requires that in order to count a collatteral vessel as such, the full length of the vessel must be identifiable: the stem, midzone and re-entry zone. Arteries were differentiated from veins, by evaluating whether the small vessell in question branched from the main arterial or was in direct connection with the main venular system. For optimal identification, collaterals were scored on rotational maximum intensity projections (MIPs) (72 partitions of 5 degrees rotation around the vertical axis) of digitally subtracted three-dimensional MRA images, using commercial software (Easyscil version 2.4.5. Philips Medical Systems, Best, The Netherlands). This method of scoring collateral vessels appeared to be superior ${ }^{21}$ over counting collateral vessels on single-directional MIPs or on original image slices, on which the smallest diameter collateral vessels cannot be traced, because collateral vessels are known to be tortuous.

\section{Histology}

Immediately following the lethal dose of pentobarbital, the anterior tibial muscle and soleus muscle samples were excised, fixed in length by pinning the muscle sample on a cork, and frozen in iso-penthane, and stored at $-80^{\circ} \mathrm{C}$. When all muscle samples were collected, $10 \mu \mathrm{m}$-thick cryosections, perpendicular to the muscle fiber direction, were stained using nitroblue tetrazolium / 5 bromo-4-chloro-3-indollylphosphate-p-toluidine salt (NBT/BCZP: Gibco, Grand Island, NY) of alkaline phosphatase in endothelial cells, as described previously by Degens et al. ${ }^{30}$. The number microvessels per muscle fiber ratio was scored independently by two observers, using optical microscopy (magnification, $200 x$ ) in three randomly selected optic fields in each muscle section. The average score of the two observers was used for further comparisons.

\section{Statistical analysis}

Differences between histologic, MRA and DCE-MRI scores in the ligated and non-ligated limb were compared using the paired Student t test. Statistical analyses were performed using commercial software (SPSS 10.0.5, SSPS, Chicago, IL). The association between MRA and $K^{P S}$ and between MRA and arterial inflow delay was quantified by robust linear regression analysis using commercial software (Stata / SE8.0, State Cooperation, College Station, Texas, USA), in which the data were clustered to account for 
dependencies between data points, given the serial measurements (same animal) and the drop-out. The level of significance was set at $p<0.05$.

\section{Results}

DCE-MRI in the lower bindlimb

Typical MR signal curves are presented in Figure 1. An example of voxel-wise $K^{p s}$ calculations for both limbs is presented as a color codied voxel map in Figure 2.
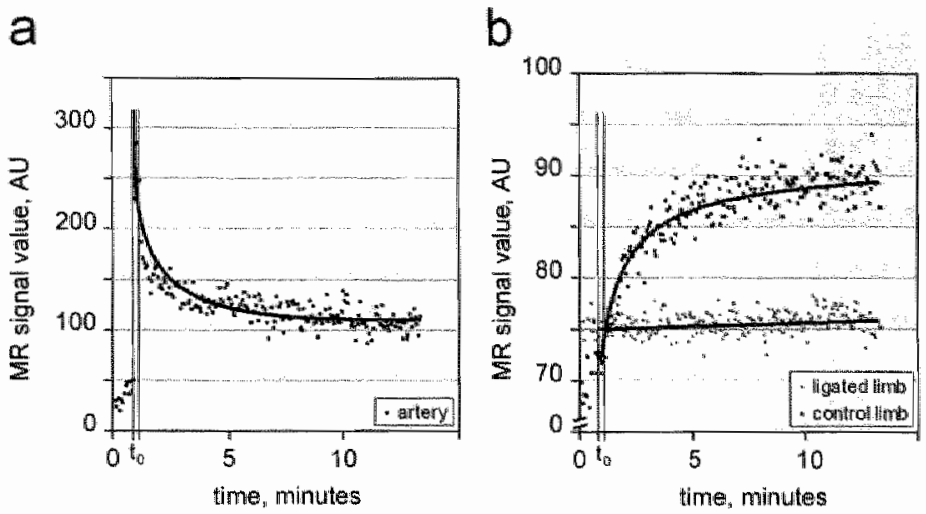

Figure 1. Typicall signal enhancement curves obtained (a) in an artery (at the aorta bifurcation) and (b) in the anterior tibial muscles in the ligated limb and non-ligated (control) limb in a rabbit a day $0^{*}$.

At day $0^{*}, K^{P S}$ in the anterior tibial muscle of the ligated limb was reduced to $23 \%$ (control limb $\pm S D$ / ligated limb $\pm S D, 9 \pm 5 / 2 \pm 2)(p=0.0008)$ as compared to the control limb (Figures 2 and $3 a$ ). This reduction was about twice as strong $(p=0.009)$ as in the soleus muscle $(63 \%(11 \pm 12 / 6 \pm 5), p=0.04)$ (Figure $3 b) . K^{P S}$ recovery by day 7 was more pronounced in the anterior tibial muscle $(7 \pm 4 / 6 \pm 2)(p<0.0003)$ than in the soleus muscle $(8 \pm 2 / 6 \pm 2)(n . s ., p>0.1)$, with respect to the $K^{p s}$ at day $0^{*}$. By day $21, K^{p 8}$ increased to within $15 \%$ (n.s. difference, $p>0.2$ ) of the control limb in both the anterior tibial- $(10 \pm 8 / 7 \pm 6)$ and soleus muscle $(11 \pm 8 / 10 \pm 5)$.

In the control limb muscles, $K^{\mathrm{PS}}$ showed no significant differences $(p>0.3)$ between day $0^{*}$, day 7 or day 21 , nor between the soleus muscles and anterior tibial muscles $(p>0.2)$ at any of the measurement intervals. 


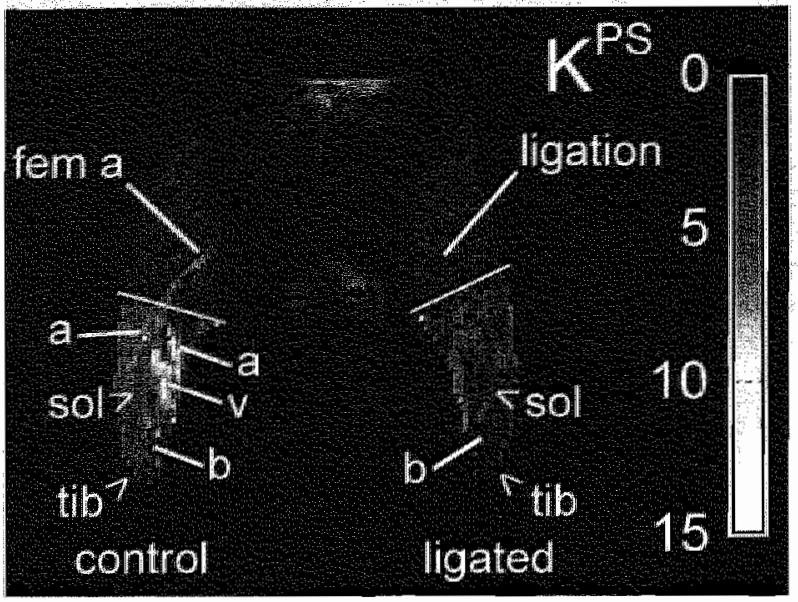

Figure 2. A representative coronal section in a rabbit two hours after femoral artery ligation (day $0^{*}$ ) with collor ${ }^{* *}$ coded $K^{P S}$ maps in the both lower hindlimbs visualize the reduction in $K^{P S}$. Arrowheads indicate the regions of the soleus muscle (sol) and anterior tibial muscle (tib). The femoral artery (fem a) enhances only in the control limb, and not in the limb with the ligated femoral artery. The $K^{p S S}$ map (units: $\mathrm{ml} \mathrm{min}^{-4} \mathrm{~cm}^{-3}$ ) also illustrates how muscle regions of interest were selected and the main arteries ( $a$ ) and veins ( $v$ ), and bone were excluded. * Color image is on the back-cover (left).

a

anterior tibial $\mathrm{m}$.

b soleus $\mathrm{m}$.
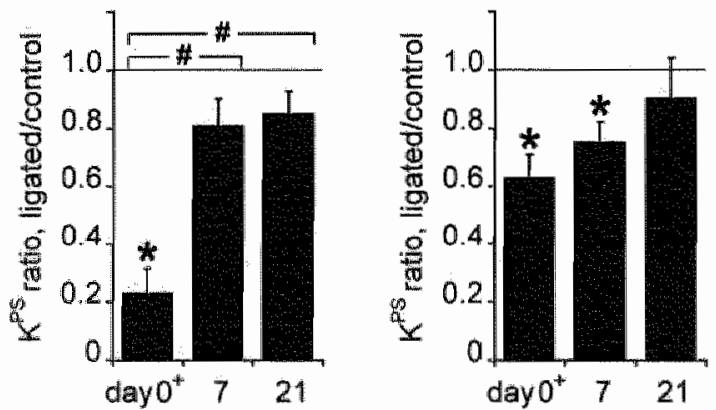

Figure 3. Median $K^{P S}$ ratios (i.e., $K^{P S S}$ in the ligated limb / $K^{P S}$ in the control limb) shows the relative changes in the anterior tibial muscle (a) and soleus muscle (b) of the serial DCE-MRI measurements. The horizontal line at $K^{\mathrm{PS}}=1$ indicates the level at which $\mathrm{K}^{\mathrm{PS}}$ would be equal in the control- and ligated limb. "Indicates significant differences $(p<0.05)$ between days $0^{*}, 7$ and 21: * indicates significant differences $(p<0.05)$ between the ligated and control limb. Error bars represent the standard error.

\section{Arterial inflow delay to the lower hindimb}

Two hours after ligation (day $\mathrm{O}^{+}$), the arterial flow to the lower ligated limb was significantly (mean $\pm S D, 9.2 \pm 3.4 \mathrm{~s} ; \mathrm{p}<0.0001$ ) delayed with respect to the control limb, and showed significant recovery by day $7(2.9 \pm 2.6 s, p=0.001)$ and normalized by day $21(<1.0 \pm 0.4 s, p<0.001)$ compared to day $0^{*}$. The arterial inflow delay in the 
control limb was consistently less than $1 \mathrm{~s}$, without significant differences $(p>0.5)$ between the measurement intervals.

Angiography of collateral growth

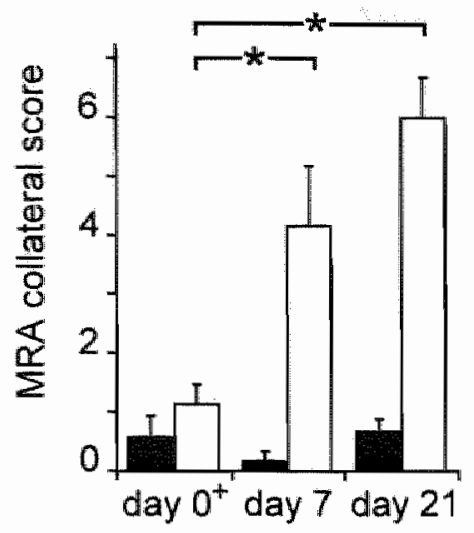

Figure 4. The (average) number of collaterals scored on MRA shows significant growth of collateral vessels from day $D^{+}$to days 7 and 21 in the ligated limb (white box plots), but not in the control limb (black box plots). Error bars represent the standard error, and the asteriste (*) indicates statistical significance at $\mathrm{p}<0.01$.

MRA showed significant collateral growth, predominantly stemming from the same vessels around the ligation and growing in a similar manner in different animals, from day $0^{+}$to day $7(p=0.01)$ and to a lesser extent from day 7 to day $21(n . s, p>0.05)$, in the ligated limb (Figure 4). The collateral score in the controllimb was consistently less than 1 at each of the three measurement intervals.

A significant inverse association was observed between MRA and arterial inflow delay $\left(R^{2}=0.33, p=0.005\right)$. A significant positive association was observed between MRA and $K^{P S}$ in the anterior tibial muscle in the ligated $\operatorname{limb}\left(R^{2}=0.42, p=0.0004\right)$ but not with $K^{P S}$ values in the soleus muscle $\left(R^{2}=0.001, p=0.96\right)$.

\section{Microvessels per muscle fiber ratio}

Histologic samples collected at day 21 showed no significant differences $(p>0.2)$ in microvessel per muscle fiber ratio between ligated-and control limb anterior tibial and soleus muscles. Microvessel per muscle fiber ratios were $71 \%$ (soleus m. ratio \pm SD / anterior tibial $m$. ratio $\pm S D, 2.4 \pm 1.0 / 1.4 \pm 0.4)(p=0.01)$ higher in the soleus muscle than in the anterior tibial muscle in the ligated limb, and $41 \%(2.4 \pm 0.9 / 1.7 \pm 0.2)(\mathrm{p}=$ 0.03 ) in the control limb (Figure 5). 


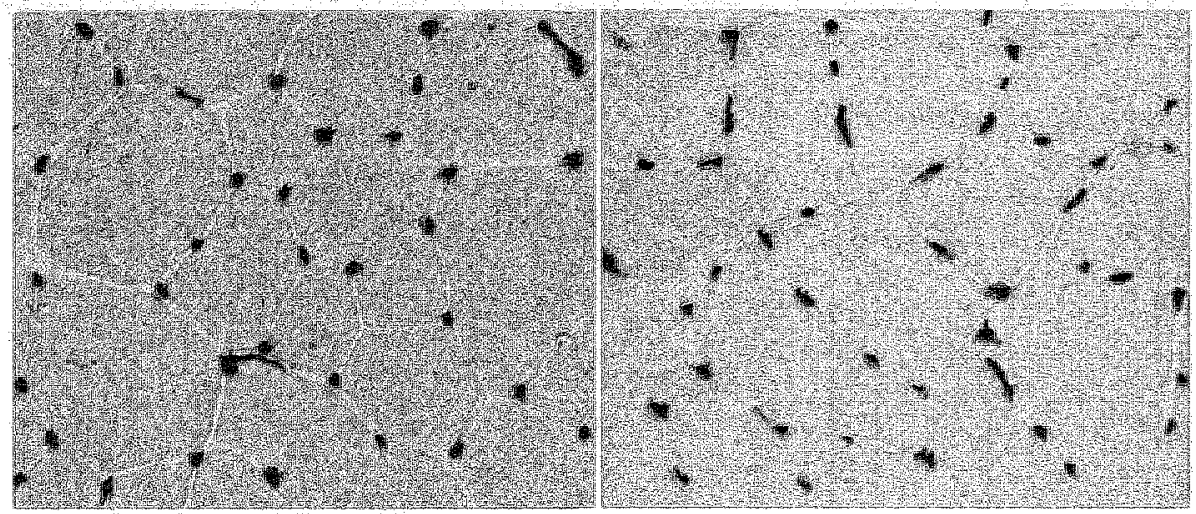

\section{anterior tibial $\mathrm{m}$.}

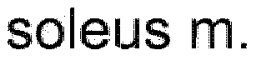

Figure 5. Microvessel staining (to alkaline phosphatase) showed that the number of microvessels per muscle fiber score was higher in soleus muscle samples than in anterior tibial muscle samples. The samples illustrated here (in gray scale) were collected from the ligated limb, in the same animal. The average scores for this animal were 2.7 vessells per muscle fiber in the soleus muscle and 1.7 vessels per muscle fiber in the anterior tibial muscle. The magnification of the above illustration is $200 x$.

\section{Discussion}

This study showed recovery of lower limb muscle perfusion, associated to collateral vessel growth, in a rabbit femoral artery ligation model, by serial MR imaging.

At present, it is unclear what techniques best allow serial non-invasive evaluation of neovascularization in vivo. The ideal technique might involve the utilization of an imaging modality that on the one hand visualizes sub-millimeter collateral vessels and on the other hand evaluates tissue perfusion, preferably in one imaging session. Of the available imaging modalities, such as X-ray angiography, ultrasound, computed tomography, single-photon-emission tomography and positron-emission tomography, the favored imaging modality may be magnetic resonance (MR) imaging ${ }^{25,34}$. As was shown here, MR imaging facilitates non-invasive and serial evaluations of both the vasculature and tissue perfusion ${ }^{32-34}$, without use of ionizing radiation, risk of nephrotoxilc reactions to ladine based contrast agents, and without the need for arterial catheterization for local contrast agent injection.

MRA visualized newly formed collateral vessels. DCE-MRI showed acute reduction and consequent recovery of blood inflow to the distal end of the hindlimb, after femoral artery ligation, as well as the acute reduction and recovery of muscle blood flow in terms of the endothelial transfer coefficient $\left(K^{\mathrm{PS}}\right)$.

An interesting finding was that the reduction in $K^{P S}$ after femoral artery ligation was more extreme in the anterior tibial muscle than in the soleus muscle. A possible explanation is that the soleus muscle has more capillaries per muscle fiber (i.e., 'red' muscle) than the anterior tibial muscle (i.e., "white' muscle), as was shown with histology. What histology intrinsically is unable to show, however, is that a large number of these muscle capillaries are not perfused during resting conditions, particularly in 'red' axidative 
muscles, and that muscles can mediate as much as a 20 -fold increase in blood flow during exercise, for example ${ }^{35}$. Muscles increase blood flow by apening up of dormant capillaries and increasing microvessel surface area. $K^{P S}$ then is enhanced by both the increase in flow and surface area ${ }^{11,35}$. Directly after ligation, the white' anterior tiblal muscle (glycolitic) had the greatest reduction in $\mathrm{K}^{\mathrm{PS}}$ owing to its limited reserve capacity. and the 'red' soleus muscle might steal the better part of the greatly limited blood supply. That the $K^{P S}$ reductions in the anterior tibial- and soleus muscles had become equivalent by day 7 might be explained by the substantial recovery in arterial inflow at day 7 . This recovery in arterial inflow might have caused the relative biood flow to retum to a more normal distribution between the soleus- and anterior tibial muscle, since the rabbits were imaged during rest. Changes in capillary recruitment ${ }^{8}$ or possibly muscle metabolism may also have played part in relative blood flow distributions after femoral artery ligation. Histologic microvessel density counts, frequently used to validate DCE-MRI in oncology 27.36, as well as the microvessel per muscle fiber ratios used here, are limited to the extent that histology renders non-physiological parameters that cannot differentiate between functional and dormant blood vessels. For example, only about $20-25 \%$ of muscle capillaries mediate blood flow under normal resting conditions ${ }^{35}$. This might explain why significant differences between soleus muscle and anterior tibial muscle observed with histology in the non-ligated limb were not observed with the $K^{P S}$ measurements.

The end-point histologic measurement offered no evidence for capillary sprouting in the lower ligated limb muscles at day 21. The capillaries per muscle fiber values observed at day 21 , correspond well with those reported by Cherwek et al. ${ }^{37}$, who also reported no significant differences in capillaries per muscle fiber between day 1 and day 5 following femoral artery ligation. While others, for example Hershey et al. ${ }^{g}$, report that skeletal muscle capillary density significantly increases at days 5 and 10, but not at days 20 and 40 after femoral artery resection (instead of ligation).

A possible relationship between MR derived parameters and capillaries per muscle fiber, for example a transient increase in the ligated limb at day 7 , which in our model seems less likely given the reports by Cherwek et al ${ }^{37}$, could not be evaluated here, because histological samples were not obtained at day 7 or day $0^{+}$. As the emphasis of our study approach was on serial (non-invasive) MR imaging, it remains unknown whether the number of capillaries per muscle fiber were increased at day 7 in the ligated limb ${ }^{\text {to }}$. Therefore, it can be concluded only that vascullar recovery in the peripheral ischemia model used here is significantly related to collateral vessel growth.

High spatial resolution MRA visualized the sub-millimeter diameter coillateral vessels that formed around the ligation. The direct association between collateral vessel scores and $K^{P S}$ in the anterior tibial muscle and the inverse association with the arterial inflow delay support that MRA allowed the visualization of functional conductance vessels. Differences in spatial resolution may explain why collateral scores reported here for MRA are lower than typically reported for high spatial resolution $X$-ray angiography ${ }^{7,8,24}$. Spatial resolution is currently a limitation of MRA on clinical MR units "which cannot facilitate spatial resolutions, comparable to $X$-ray, without compromising anatomic coverage or acquisition duration ${ }^{38}$. Ongoing developments in MR imaging units, MR receiver coils, contrast agents, and imaging protocols might further improve visualization of collateral growth by (serial) MRA ${ }^{24}$. 


\section{Practical application}

Further work, including disease (e.g. diabetic) models may show whether DCE-MRI and MRA combined is indeed successful for evaluating peripheral arterial disease and the effects of treatments that stimulate vessel development and growth. 


\section{References}

1. Post M, Laham $R_{b}$ Sellke $F W_{1}$ Simons $M$. Therapeutic angiogenesis in cardiology using protein formulations. Cardiovasc Res. 2001,49:522-31.

2. Collinsan $\mathrm{D}$; , Donnelly $\mathrm{R}$. Therapeutic angiogenesis in peripheral arterial disease: can biotechnollogy produce an effective collateral circulation? Eur J Vasc Endovase Surg. 2004,28:923.

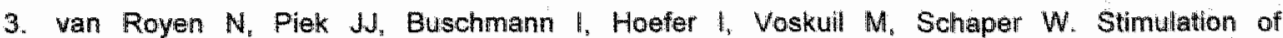
arteriogenesis; a new concept for the treatment of arterial occlusive disemse. Cardiovasc Ros. 2001:49:543-53.

4. Carmeliet $P$. Mechanisms of angiogenesis and arteriogenesis. Nat Med. 2000;6:389-95.

5. Pipp F, Heil M, Issbrucker $K$, Ziegelhoeffer $T$, Martin $S$, van den Heuvel J, Weich H, Fernandez B, Golomb $G$, Carmeliet $P$, Sichaper W. Clauss M. VEGFR-1-selective VEGF homologue PIGF is arteriogenic: evidence for a monocyte-mediated mechanism. Circ Res. 2003;92:378-85.

6. Buschmann 1, Heil $M$, Jost $M$, Schaper $W$. Inftuence of inflammatory cytokines on arteriogenesis. Microcirculation. $2003,10: 371-9$.

7. Hoefer $I E$, van Royen $N$, Buschmann $\mathbb{R}$, Piek $J J$, Schaper W. Time course of arteriogenesis following femoral artery occlusion in the rabbit. Cardiovasc Res. 2001; 49:609-17.

8. Zakrzewicz A, Secomb TW, Pries AR. Angioadaptation: keeping the vascular system in shape. News Physiol Sci. 2002;17:197-201.

9. Hershey JC, Baskin EP, Glass JD, Hartman HA, Gilberto DB, Rogers IT, Cook Jل. Revascularization in the rabbit hindlimb: dissociation between capillary sprouting and arteriogenesis. Cardiovasc Res. 2001;49:618-25.

10. Arras M, Ito WD, Scholz D, Winkler B, Schaper J, Schaper W. Monocyte activation in angiogenesis and collateral growth in the rabbit hindlimb. 1 Clin Inwest. 1998;101:40-50.

11. Cao $R$, Brakenhielm $E$, Pawliuk $R$, Wariaro $D$, Post $M J$, Wahiberg $E$, Leboulch $P$, Caio $Y$. Angiogenic synergism, vascular stability and improvement of hind-limb ischemia by a combination of PDGF-BB and FGF-2. Nat Med, 2003;9:604-13.

12. Pearlman JD, Laham RJ, Simons M. Coronary angiogenesis: detection in wivo with MR imaging sensitive to collateral neocirculation-preliminary study in pigs. Radiology. 2000;214:801-7.

13. Pearlman JD, Hibberd MG, Chuang ML, Harada $K$, Lopez JJ, Gladstone SR, Friedman M, Sellke FW. Simons M. Magnetic resonance mapping demonstrates benefits of VEGF-induced myocardial anglogenesis. Nat Med. 1995;1:1085-9.

14. Jackson $A_{n}$ Buckley DL, Parker GJM. Dynamic contrast-enhanced magnetic resonance imaging in ancology. \& ed. Heidelberg, Germany: Springer-Verlag; 2005.

15. Leach MO, Brindle KM, Evelhoch JL, Griffiths JR, Horsman MR, Jackson A, Jayson G, Judson IR, Knopp MV, Maxwell RJ, Mcintyre D, Padhani AR, Price P, Rathbone R, Rustin G, Tofts PS, Tozer $G M$, Vennart $W$, Waterton JC, Williams $S R$, Workman $P$. Assessment of antiangiogenic and antivascular therapeutics using MRI: recommendations for appropriate methodology for clinical trials. Br J Radiol. 2003;76 Spec No 1:S87-91.

16. Padhani AR. Dynamic contrast-enhanced MRI in clinical oncology: Current status and future directions. J Magn Reson lmaging. 2002;16:407-22.

17. de Lussanet QG, Beets-Tan RG, Backes WH, van der Schaft DW, van Engelshoven JM, Mayo $\mathrm{KH}$, Grifficen AW. Dynamic contrast-enhanced magnetic resonance imaging at 1.5 Tesla with gadopentetate dimeglumine to assess the angiostatic effects of anginex in mice. Eur $J$ Cancer. 2004:40:1262-8.

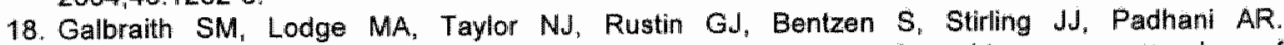
Reproducibility of dynamic contrast-enhanced MRI in human muscle and tumours: comparison of quantitative and semi-quantitative analysis. NMR Biomed. 2002;15:132-42.

19. de Lussanet $Q G$, Langereis $S$, Beets-Tan RG, van Genderen $M H$, Griffioen AW, van Engelshoven $J M$, Backes. WH. Dynamic Contrast-enhanced MR Imaging Kinetic Parameters and Molecular Weight of Dendritic Contrast Agents in Tumor Angiogenesis in Mice. Radiology. 2005.

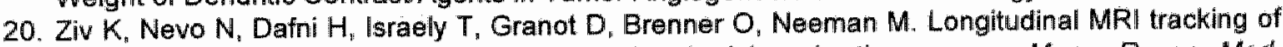
the angiogenic response to hind limb ischemic injury in the mouse. Magn Reson Med. 2004:51:304-11.

21. de Lussanet QG, van Golde JCG, Beets-Tan RGH, de Haan MW, Zaar DVJ, Post MJ, Huiberts MS, Schaper NC, van Engelshoven JMA. Backes WH. Magnetic resonance angiography of 
collateral vessel growth in a rabbit femoral artery ligation model. MMR in Biomedicine. 2005;in press.

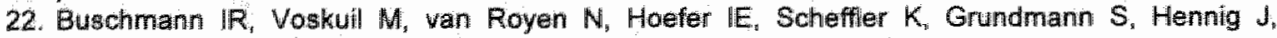
Schaper W. Bode $\mathrm{C}$. Piek J.J. Invasive and non-invasive evaluation of spontaneous arteriogenesis in a novel porcine model for peripheral: arterial obstructive disease. Atherosclerosis. 2003; 167:3343.

23. Wecksell MB, Winchester PA, Bush HL, Jr., Kent KC, Prince MR, Wang $Y$. Cross-sectional patteri of collateral vessels in patients with superficial femorall artery occlusion. Invest Radiol. $2001 ; 36: 422-9$.

24. Wagner $S$, Helisch $A$, Ziegelhioffer T, Bachmann G, Schaper W. Magnetic resonance angiography of collateral vessels in a murine femoral artery ligation model. NMR Biomed. $2004: 17: 21-7$.

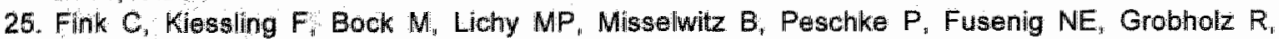
Delorme S. High-resolution three-dimensional MR angiography of rodent tumors: morphologic characterization of intratumoral vasculature. I Magn Resan Imaging. 2003;18:59-65.

26. Haacke $M$. Brown RW, Thompson MR, Venkatesan $R$. T1 estimation from SSI measurements at multiple flip angles. In: Haacke $M$, Brown RW, Thompson MR, Venkatesan $R_{s}$ eds. Magnetic resonance imaging: physical principles and sequence design. 1 ed. New York "NY: John Wiley \& Sons, Inc.; 1999:654-661.

27. de Lussanet QG, Backes WH, Griffioen AW, van Engelshoven JM, Beets-Tan RG. Gadopentetate dimeglumine versus ultrasmall superparamagnetic iron oxide for dynamic contrast-enhanced MR imaging of tumor angiogenesis in thuman colon carcinoma in mice. Radiology. 2003;229:429-38.

28. Daldrup HE, Shames DM, Husseini W. Wendland MF, Okuhata $Y$, Brasch RC. Quantification of the extraction fraction for gadopentetate across breast cancer capillaries. Magn Reson Med. $1998 ; 40: 537-43$.

29. Longland C.J. The collateral circulation of the limb; Arris and Gale lecture delivered at the Royal College of Surgeons of England on 4th February, 1953. Ann R Coll Surg Engl. 1953;13:161-76.

30. Degens $H_{1}$ Turek $Z$, Hoofd LJ, Van't Hof MA, Binkhorst RA. The relationship between capillarisation and fibre types during compensatory hypertrophy of the plantaris muscle in the rat. UAnat. 1992;180 (Pt 3):455-63.

31. McDonald DM, Choyke PL. Imaging of angiogenesis: from microscope to clinic. Nat Med. 2003;9:713-25.

32. Liu $Y$, Karonen JO, Vanninen $R L$, Nuutinen J, Koskela $A_{*}$ Soimakallio $S$, Aronen HJ. Acute ischemic stroke: predictive value of $2 D$ phase-contrast MR angiography--serial study with combined diffusion and perfusion MR imaging. Radiology. 2004;231:517-27.

33. Baumgartner I, Thoeny $H C$, Kummer $O$, Roefke $C$, Skjelswik $C$, Boesch $C$, Kreis R. Leg Ischemia: Assessment with MR Angiography and Spectroscopy. Radiology. 2005;234:833-41.

34. Waller $C$, Hiller $K H$, Albrecht $M$, Hu $K$, Nahrendorf $M$, Gatteniohner $S$, Haase $A$, Erti $G$, Bauer WR. Microvascular adaptation to coronary stenosis in the rat heart in vivo: a serial magnetic resonance imaging study. Microvase Res. 2003;66:173-82.

35. Guyton $\mathrm{AC}$. Muscle blood flow and cardiac output during exercise. In: Wonsiewicz $M J_{\text {n }}$ ed. Textbook of Medical Physiology. 8 ad. Philadelphia, PA: W. B. Saunders Company: 1991:234237.

36. van Dijke CF, Brasch RC, Roberts TP, Weidner N, Mathur A, Shames DM, Mann JS, Demsiar F, Lang $P$, Schwickert HC. Mammary carcinoma model: correlation of macromolecular contrastenhanced MR imaging characterizations of tumor microvasculature and histologic capillary density. Radiology. 1996;198:813 4 .

37. Cherwek DH, Hopkins MB, Thompson MJ, Annex BH, Taylor DA. Fiber type-specific differential expression of anglogenic factors in response to chronic hindlimb ischemia. Am J Physiol Heart Circ Physiol. 2000,279:H932-8.

38. Nijenhuis RJ, Leiner T, Cornips EM, Wilminik JT, Jacobs MJ, van Engelshoven JM, Backes WH. Spinal cord feeding arteries at MR anglography for thoracoscopic spinal surgery: feasibility study and implications for surgical approach. Radiology. 2004;233:541-7. 


\title{
Chapter 7
}

\section{Magnetic resonance angiography of collateral vessel growth in a rabbit femoral artery ligation model}

\author{
NMR in Biomedicine, 2005 In press
}

\begin{abstract}
Quido G. de Lussanet, Jolanda C.G. van Golde, Regina G.H. Beets-Tan, Michiel W. de Haan, Daniëlle V.J. Zaar, Mark J. Post, Maya S. Huijberts, Nicollaas C. Schaper, Jlos M.A. van Engelshoven, Walter H. Backes.
\end{abstract}

\section{ABSTRACT}

\section{PURPOSE}

Collateral vessel growth was visualized in a rabbit femoral artery ligation model by serial contrast-enhanced magnetic resonance angiography (MRA) at 1.5 Tesla in comparison with $X$-ray angiography (XRA).

\section{MATERIALS AND METHODS}

XRA and MRA were performed directly after femoral artery ligation (day $0^{+}$) and after 7 and 21 days. XRA (in-plane resolution, $0.3 \times 0.3 \mathrm{~mm}$ ) was performed with arterial catheterization for fast injection of iodinated contrast agent just proximal to the aortic bifurcation. MRA (in-plane, $0.6 \times 0.6 \mathrm{~mm}$ ) was performed at 1.5 Tesla with a 5-element phased-array coil, and slow injection of gadolinium based MR contrast agent into an ear vein. Collateral vessel scores on two-dimensional XRA projections, and on threedimensional digitally subtracted rotational MRA maximum intensity projections were obtained by two observers, and compared. Collateral vessel counts and minimal detectable vessel diameters for MRA and XRA were combined in a computational flow model to interpret differences in spatial detection limits between imaging modalities in terms of flow.

\section{RESULTS}

Collateral vessel scores were significantly higher in the ligated limb at day $7(p<0.05)$ and more so at day $21(\mathrm{p}<0.001)$, in comparison to day $0^{+}$or in the non-ligated control limb on both XRA and MRA. Significantly more (smaller) vessels were visualized with XRA than with MRA, particularly on day $21(p<0.05)$. Interobserver agreement was high for both XRA $(\kappa=0.82)$ and MRA $(\kappa=0.78)$. The flow model showed that collateral vessels with diameters $>0.3 \mathrm{~mm}$ scored by XRA as well as MRA represent nearly $100 \%$ of the total blood flow, while smaller $(0.1-0.3 \mathrm{~mm}$ diameter) vessels that can only be detected with XRA contribute little to the blood flow.

\section{CONCLUSION}

Serial contrast-enhanced MRA can non-invasively visualize sub-millimeter collateral vessels that represent nearly $100 \%$ of the restored blood flow, in a femoral artery ligation model. 


\section{Introduction}

Collateral vessel growth can be visualized in vivo by high-spatial resolution imaging, as was shown for example in femoral artery ligation models for peripheral arterial disease ${ }^{\text {t- }}$

${ }^{3}$. After femoral artery ligation, pre-existent arterioles around the ligated arterial segment will remodel and increase in diameter, either spontaneously or in response to therapy, to become mature conductance vessels ${ }^{2,4-7}$. This collateral vessel growth is demonstrable in the first weeks following femoral artery ligation by high spatial resolution $X$-ray angiography (XRA) ${ }^{1,2}$. In vivo XRA, however, involves arterial cannulation for (highpressure) injection of lodinated contrast agent and ionizing radiation exposure, which complicates serial evaluation with XRA. Magnetic resonance angiography (MRA) does not have these disadvantages. Theoretically, MRA would therefore be an ideal modality for serial in vivo evaluation of collateral vessel growth, provided that the spatial resolution is high enough to visualize sub-millimeter sized collateral vessels ${ }^{8}$. In terms of spatiai resolution XRA is generally superior over MRA. Contrary to conventional XRA. systems, MRA can readily produce three-dimensional image data (instead of fixed twodimensional projection images) that can be post-processed to study the territories and morphology of collateral growing vessels. Three-dimensional sub-millimeter spatial resolution MRA does require a relatively long acquisition time, which results in high background signal and venous enhancement. Therefore spatial resolution of MRA should not only be sufficient to allow visualization of collateral vessels but should also distinguish between arteries and veins.

To evaluate whether serial contrast-enhanced MRA with relatively long acquisition time and sub-millimeter spatial resolution visualizes collateral artery development, we compared collateral vessel growth visualized by serial MRA with selective XRA in a rabbit femoral artery ligation model. As the spatial resolution of MRA is lower than for XRA, larger diameter and, therefore, fewer collateral vessels are expected to be detectable by MRA. A priori it remains unknown how the (possibly) numerous small collaterals contribute to the blood flow in comparison to the (likely) less numerous larger diarneter collaterals. To relate the collateral vessels that are detected to expected recovery of blood flow, collateral vessels scores and spatial resolutions (i.e., minimal detectable vessel diameter) for MRA and XRA were interpreted in terms of flow contributions by using a simple computational flow model ${ }^{9}$.

\section{Materials and Methods}

\section{Experimental design and animals}

This study was approved by the Maastricht University animal ethics committee. Ten New Zealand White rabbits (male; weight, range $2.9-3.3 \mathrm{~kg}$ ) were included for the study. Different numbers of animals underwent XRA directly after femoral artery ligation (day $\left.0^{+}\right)(n=6)$ and day $7(n=6)$, and at day $21(n=6)$. MRA was performed at 2 hours after femoral artery ligation (day $\left.0^{*}\right)(n=7)$, day $7(n=6)$, and day $21(n=6)$ (Table 1). Two animals died between day $0^{+}$and day 7 , and two more died after day 7 . XRA failed in two animals at day $0^{*}$ owing to technical problems, and in one of these animals MR imaging was also not performed. To correct for drop out and to obtain a balanced number of data points at all time points, two animals were imaged only at day 21.

During the experiments the animals were ventilated with isoflurane (2-3\%). Occlusion (length, 1-2 cm) of the left femoral artery (day 0), and the XRA series were performed 
under sterile conditions, and a heating pad was used to maintain body temperature between 36 and $37^{\circ} \mathrm{C}$. Buprenorphine was given (intra-muscular injection) as postoperative analgesia for 3 days after the measurements at day $0^{+}$and 7 . Directly after the final MR measurement at day 21, a lethal dose of pentobarbital (1 ml/kg body weight) was injected intravenously.

\section{XRA}

Selective coronal XRA series (12 frames/s) were acquired using a portable X-ray system (BV Pulsera, Philips Medical Systems, Best, The Netherlands) (in-plane resolution $0.3 *$ $0.3 \mathrm{~mm}$, operated at tube voltage $72 \mathrm{kV}$ ), and bolus injection ( $5 \mathrm{~mL} / \mathrm{s}$ ) of non-ionic lodine contrast agent ( $240 \mathrm{mg}$ lodine $/ \mathrm{mL}, 1.6 \mathrm{~mL} / \mathrm{kg}$ body weight) (Omnipaque, Amersham Health, Eindhoven, The Netherlands) through a catheter ( $4 F$ ) inserted via the carotid artery and placed $2-3 \mathrm{~cm}$ proximal to the abdominal aorta bifurcation. XRA measurements were digitally stored for later analysis. The contrast-enhanced acquisitions were not post-processed with digital subtraction of the pre-contrast 'mask' images because collateral vessels were more difficult to score on subtraction images; possibly owing to minimal motion of the rabbit, table, and/ or portable X-ray unit. A linepair phantom was used to demonstrate that spatial resolution was approximately $0.3 \mathrm{~mm}$ (i.e., 2 line pairs per $\mathrm{mm}$ ).

\section{MRA}

MRA was performed using a clinical 1.5 Tesla MRI system (Philips Intera, Philips Medical Systems, Best, The Netherlands) with a maximum gradient strength $30 \mathrm{mT} / \mathrm{m}$ and rise time of $200 \mu \mathrm{s}$. The MRI system was equipped with a 5-element phased array coill (Synergy Cardiac, Philips Medical Systems). Prior to obtaining high-resolution; contrast-enhanced MR angiograms, a reference MR acquisition was performed to allow correction of spatial inhomogeneity (Constant Level AppeaRance (CLEAR), Philips Medical Systems). The MRA protocol consisted of a pre-contrast acquisition (i.e. mask) and a contrast-enhanced acquisition, with identical imaging parameters. This was a three-dimensional T1-weighted spoiled gradient recalled echo (fast field-echo per Philips terminology) pulse sequence with echo time / repetition time $2.5 / 9.5 \mathrm{msec}_{\text {; }}$ flip angle $30^{\circ}$, field-of-view $196 \times 176 \mathrm{~mm}$, matrix size $326 \times 294$, centric $k$-space filling, resulting in a (acquired) voxel size of $0.6 \times 0.6 \times 0.7 \mathrm{~mm}^{3} .80$ coronal sections were obtained and the duration was 2.5 min per acquisition. Eight seconds before starting the contrastenhanced MRA acquisition gadopentetate dimeglumine $(0.2 \mathrm{~mL} / \mathrm{kg}$ body weight) was slowly injected (injection rate, $1 \mathrm{~mL} / 30 \mathrm{~s}$ ) in an ear vein and flushed (1 $\mathrm{mL} / 10 \mathrm{~s}$ ) with $2 \mathrm{~mL}$ saline. The scan delay of 8 seconds was determined in a previous pilot study using high temporal resolution $(\sim 2 \mathrm{~s}$ ) dynamic contrast-enhanced MR pulse sequences (data not shown), and served to enhance (first bolus pass) arterial conspicuity by synchronizing the start of the centric k-space acquisition with the contrast bolus arrival at the aortic bifurcation. After completion of the acquisition, the mask image data set was subtracted from the contrast-enhanced data set to increase vessel-to-background contrast, and to enhance small vessel conspicuity. No heating pad was used during MRI and the average (rectal) temperature measured in the animals directly after MRI was $35.5 \pm 0.5$ C.

\section{Data analysis}

The number of collaterals were counted by two independent observers using the Longland definition ${ }^{10}$, which requires identification of the stem, midzone, and re-entry 
zone. Arteries were differentiated from veins on MRA images, by evaluating whether the small vessel in question branched from the main arterial or was in direct connection with the main venular system. Vessels that could not be followed over their entire length from stem- to re-entry zone (i.e., non-continuous vessels) were not scored, because it would not be possiblle to validate whether these represent collateral arteries, small veins or small arterfes feeding a muscle. Blinding for the ligated or non-ligated control limb was not possible because the ligation was too evident on the MRA and XRA images. XRA projections were scored one month after completing the XRA and MRA measurements. The observers were blinded for animal number and date of measurement by having the projections re-coded by a third investigator. MRA images were scored with a delay of one month after the XRA images were scored. XRA series were analyzed on a diagnostic computer workstation using commercially available DICOM reader softwaire (eFilm Lite, version 1.9.2, eFilm Medical Inc Merge Technologies Inc, Milwaukee, WI). MRA images were analyzed using commercial software (Easyscil, version 2.4.5, Philips Medical Systems, Best; The Netherlands) on digitally subtracted three-dimensional rotational maximum intensity projections (MIPs) (72 partitions of 5 degrees rotation around the vertical axis) of the vessels of interest.

\section{Computational flow model}

Collateral vessels are known to grow from pre-existent arteriolles " which are too small to be visualized by MRA or XRA. As these collateral vessels grow in diameter they will first become visible only with XRA and later also with MRA. The primary reason that collateral blood vessels first appear on XRA is that it has a higher spatial resolution than MRA. The a priori difference in the number of observable vessels between XRA and MRA is unknown but can be measured. It is also unknown what this difference means in terms of contributions to muscle blood flow. To address this question, a simple laminar flow model was developed in which the distribution of collateral vessels detected by MRA and XRA was rellated to collateral blood flow. Assuming that the numbers of collateral vessels visible on MRA and XRA primarily depend on spatial resolution (i.e., minimal detectable vessel diameter), an exponentially decreasing density function $n(x)=$ $\left(n_{0} / \mu\right) \cdot \exp (-x / \mu)$ was derived to determine the number of vessels with a diameter equal to $x$. In this expression for $n(x)$, no represents the total number of vessels and $\mu$ is the average vessel diameter. According to Poiseuille's law, the flow contribution $q(x)$ of one vessel with diameter $x$ is proportional to the fourth power of the diameter (i.e., $\left.q(x)-x^{4}\right)$, assuming laminar flow pattern and equal blood pressure difference per unit vessel length, which is independent of the vessel diameter ${ }^{9}$. The total flow per vessel diameter is proportional to the product of the vessel density and flow i.e. $n(x) \cdot x^{4}$ (Figure 1). The parameters of the density function $\left(n_{0}\right.$ and $\mu$ ) were derived from the number of vessels scored by both imaging modalities on day 7 and 21 and the assumed spatial resolutions. For parameter estimation we used the cumulative density function $N(x)=n_{0} \cdot \exp (-x / \mu)$. which represents the number of vessels with a diameter larger than $x$. The parameters $n_{0}$ and $\mu$ were used to calculate the cumulative flow distribution $Q(x)$ for vessels with a diameter larger than $x$, and to estimate the contribution of vessels with different diameters, visible on MRA and XRA, to the total collateral blood flow (Fig. 1). Spatial detection limits for small contrast enhanced vessels are lower than the voxel or pixel size of the imaging technique due to the partial volume effect, and were estimated to be 0.1 $\mathrm{mm}$ (one third of the spatial resolution) for XRA and $0.3 \mathrm{~mm}$ for MRA ${ }^{11}$. 


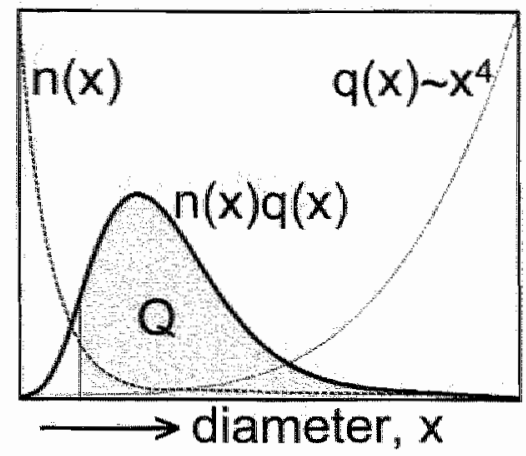

Figure 1. Diagrammatic representation illustrates that the number of callateral vessels for given diameter $n(x)$ decreases exponentially when the vessel diameter increases, while the flow within one vessel $q(x)$ increases to the fourth power with the vessel diameter for a laminar flow pattern; according to Poiseuille's law. The product of vessel size density and flow, $n(x)$; $q(x)_{\text {" }}$ is the vessel diameter specific contribution to the total blood flow, which initially increases as the diameter increases and decreases with few or zero collateral vessels with large diameters.

Note that the diameter specific flow contribution, in $(n(x) \cdot q(x))$, is highest for the very few number of vessels with a relatively large diameter (i.e., when $n(x)$ is approaching zero and $q(x)$ is elevating from zero). The cumulative flow $Q(x)$ is represented by the (shaded) area under the curve $(n(x), q(x))$. The diagram is schematic, as different quantities have different units $\left(e . g, x\right.$ in $1 \mathrm{~mm}, n(x)$ in $\| / \mathrm{mm}_{n} q(x)$ in $\mathrm{ml} \mathrm{cm} \mathrm{cm}^{-3} \mathrm{~s}^{-1}$.

\section{Statistical analyses}

Differences on day $0^{*}$, day 7 , and day 21 between XRA and MRA scores in the ligated and non-ligated limb were compared using the Mann-Whitney test. Linear weighted Cohen's $k$ was calculated to determine the inter-observer agreement ${ }^{12}$. These statistical analyses were performed using commercial software (SPSS 10.0.5, SSPS, Chicago, IL). The association between XRA and MRA scores was quantified by robust linear regression anallysis, using commercial software (Stata / SE 8.0. Stata Cooperation, College Station, Texas, USA), in which the data were clustered to account for dependencies (i.e. repeated measurements in the same animal) between data points. The level of statistical significance was set at $p<0.05$.

Table $1 \quad$ Animal inclusion

\begin{tabular}{lllll}
\hline Animal & day 0 & day $0+$ & day 7 & day 21 \\
1 & ligation & no imaging & no imaging & XRA \& MRA \\
2 & ligation & no imaging & no imaging & XRA \& MRA \\
3 & ligation & XRA \& MRA & XRA \& MRA & XRA \& MRA \\
4 & ligation & XRA \& MRA & died & \\
5 & ligation & XRA \& MRA & died & \\
6 & ligation & XRA \& MRA & XRA \& MRA & died \\
7 & ligation & no imaging & XRA \& MRA & died \\
8 & ligation & XRA \& MRA & XRA \& MRA & XRA \& MRA \\
9 & ligation & XRA \& MRA & XRA \& MRA & XRA \& MRA \\
10 & ligation & Only MRA & XRA \& MRA & XRA \& MRA \\
\hline
\end{tabular}




\section{Results}

\section{XRA and MRA}

Serial XRA and MRA visualized significant collateral vessel growth 7 and 21 days after femoral artery ligation (Figures 2 and 3). The average number of collaterals (Figures 4 and 5) in the ligated limb was significantly higher on day $7(p<0.05)$ and more so on day $21(p<0.001)$ when compared to day $0^{*}$. The numbers of collateral vessels were also significantly higher when compared to the non-ligated control limb, both on $X R A$ (day 7, p < 0.01; day $21 \mathrm{p}<0.001$ ) and MRA (day 7, $<<0.01$; day 21, $p<0.0001$ ), except for day $0^{*}$ ( $p>0.05$ ). The differences in collateral scores on day 7 and day 21 on XRA and MRA were not significant $(p>0.05)$. No significant differences $(p>0.05)$ were observed between the day $0+$, day 7 and day 21 measurements in the control limb (Figure 5). A significant association was observed between XRA and MRA scores $\left(R^{2}=\right.$ $0.34, p<0.01)$. A significant $(p<0.05)$ difference was observed between the XRA and MRA scores in the ligated limb at day 21.

Interobserver agreements were good for both XRA $(k=0.82)$ and MRA $(\kappa=0.78)$.

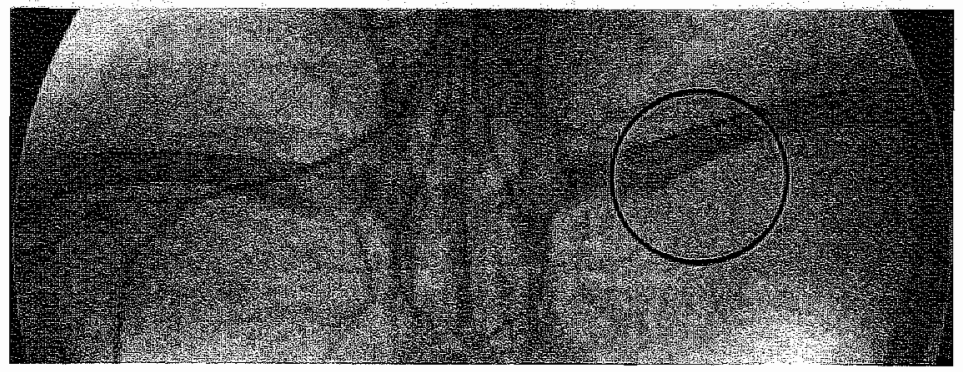

Figure 2. Serial XRA shortly after femoral artery ligation (day $0+$ ) shows the obstructed arterial flow in the left femoral artery of the ligated limb (circle) day $0^{+}$ compared with the right femoral artery

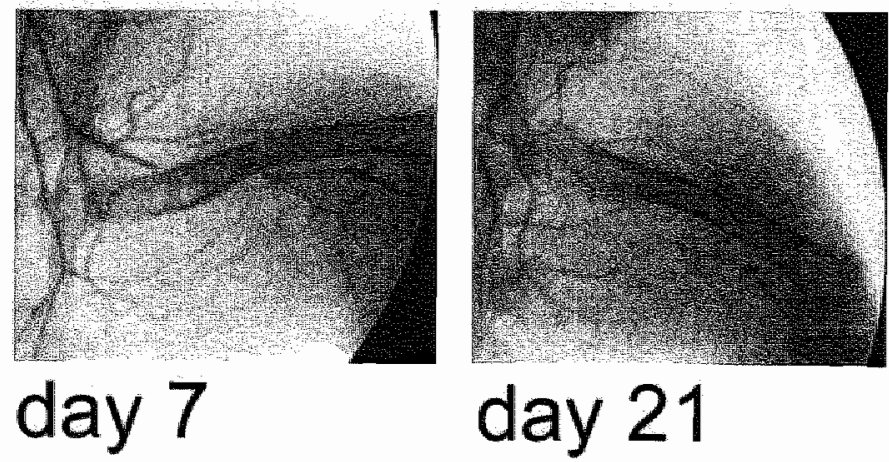
in the non-ligated limb. On day 7 collateral vessels appear to restore arterial flow distal to the ligation. The number of collateral vessels increased by day 21 .

\section{Flow model}

The cumulative density functions describing the number of vessels wider than diameter $x$ are shown in Figure $6 a$, and the calculated parameters were $n_{0}=11.5_{*} \mu=0.32 \mathrm{~mm}$ for day $T_{n}$ and $n_{0}=15.3, \mu=0.32 \mathrm{~mm}$ for day 21 , respectively. Figure $6 \mathrm{~b}$ shows the resulting contributions to the flow. The ratio of the total flow between day 7 and 21 was estimated to be 0.53 . Collateral vesisels as observed by MRA $(x>0.3 \mathrm{~mm}$, based on 
phantom experiments ${ }^{11}$ ) were estimated to contribute more than $99.5 \%$ to the total flow on day 7 as well as on day 21 .
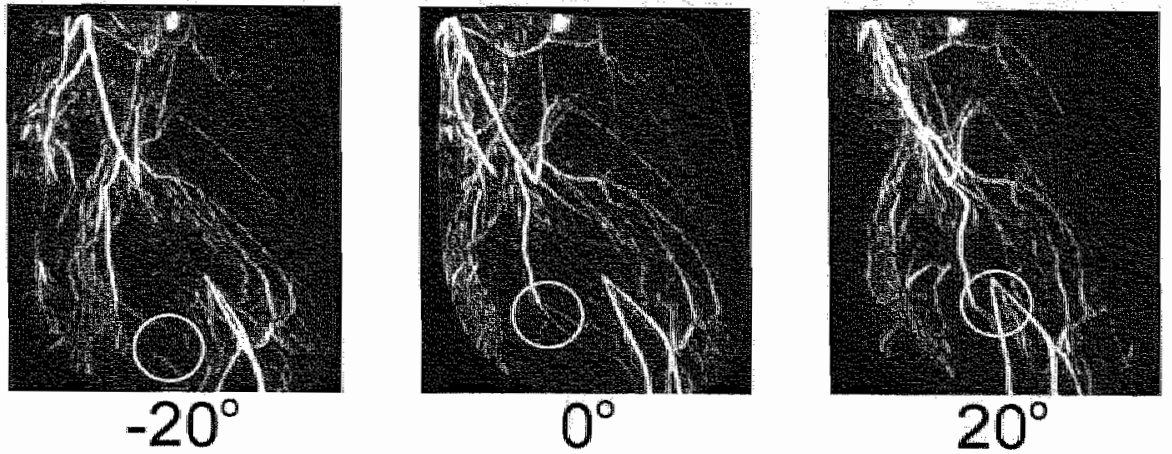

Figure 3. Serial subtraction MRA images in one rabbit (a) directly after ligation and 7 and 21 days after ligation (arrowhead) illustrate the growth of collateral vessels near the site of ligation ${ }_{\text {n }}$ and (blood) flow recovery to the distal arteries in the ligated limb (horizontal arrows), with respect to the control limb. Subtraction MRA images were reconstructed to 72 three-dimensional rotational maximum intensity projections (b) (interval, $5^{\circ}$ rotation) to score arterial collateral vessels. Illustrated here is a rabbit 21 days after ligation: the mid-zone of two medial collateral vessels is best observed at $-20^{\circ}$ rotation (circle), the stem at $0^{\circ}$ (circle) (is the anterior-posterior view), and the re-entry zone at $+20^{\circ}$ rotation (circle) (according to the Longland definition).

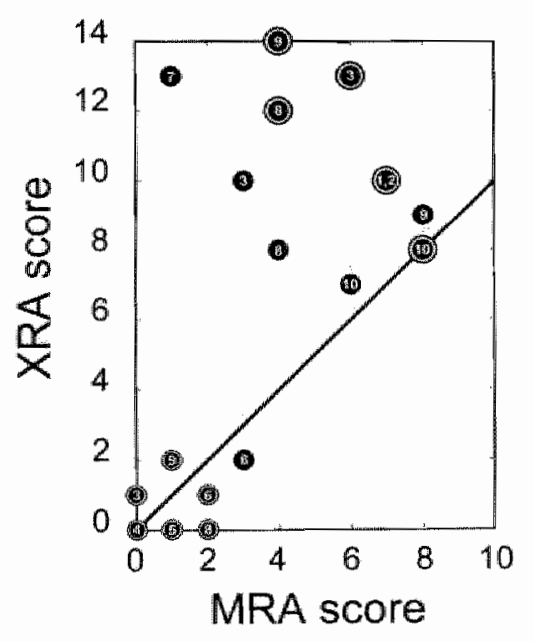

Figure 4. XRA versus MRA scores of the ten rabbits (labeled 1-10) illustrates the significant increase of the number of collaterals detected by the two imaging modalities, but also shows that XRA visualizes more collaterals than MRA as the numbers of collateral vessels increases (i.e. the XRA data points lie above the identity line). The small double circles represent the day $0+$ measurements, the solid circies represent day 7 , and the large double circles represent day 21 . 

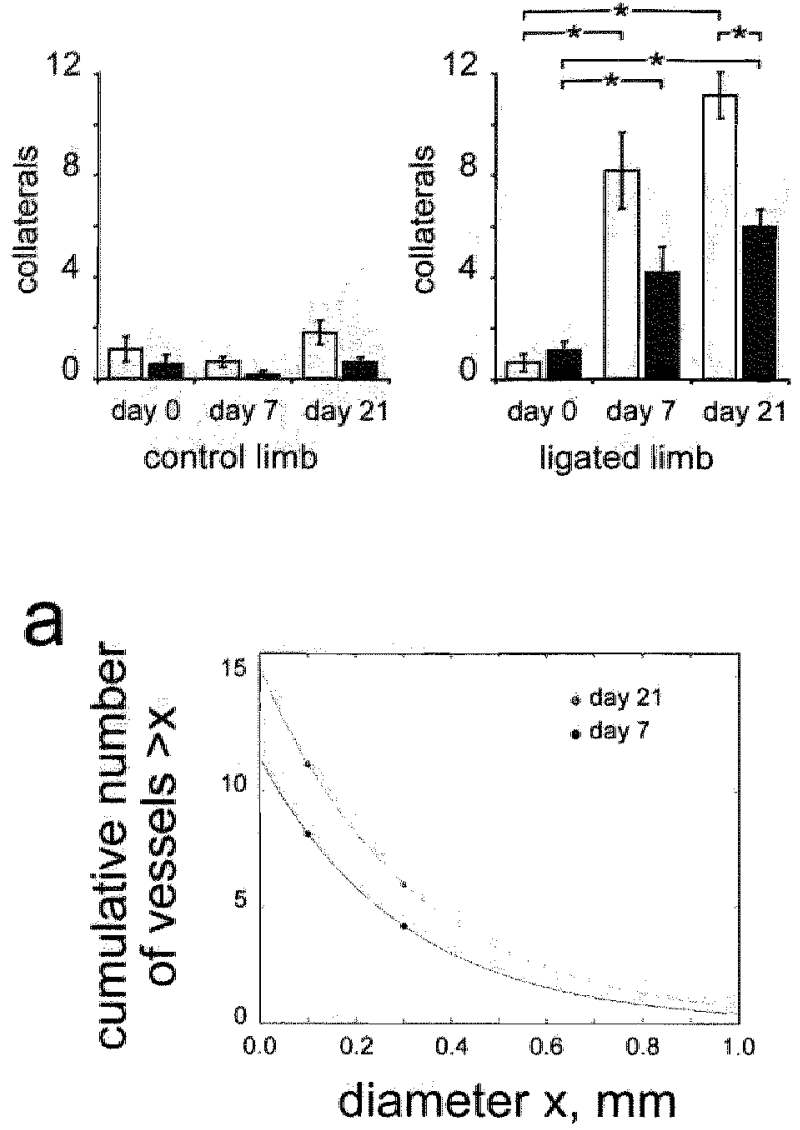

b

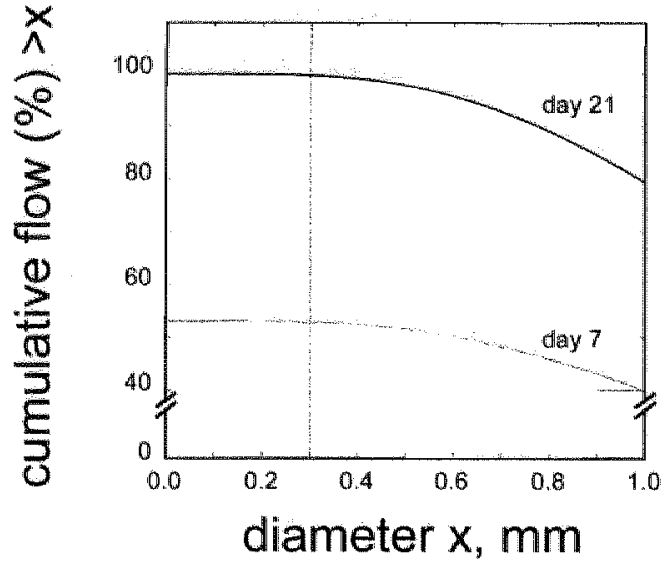

Figure 5. The average number of collaterals observed on both XRA (white) and MRA (black) increased significantly $\left({ }^{*}, p<\right.$ $0.05)$ by days 7 and 21 following ligation in the ligated limb, but not in the control limb, with a significant difference between MRA and XRA scores at day 21. Error bars represent the standard error of the mean.

Figure 6. (a) The determined minimal detectable vessel diameters of $>0.3 \mathrm{~mm}$ (vertical dotted lines) for MRA ${ }^{11}$ and $>0.1$ $\mathrm{mm}$ for XRA and the MRA and XRA collateral vessel counts on day 7 and 21 were used to numerically fit the exponential cumulative vessel diameter density function (a). (b) Subsequent calculations on the consequent cumulative flow distributions as a function of collateral vessel diameter, reveal that the collateral vessel observed with a minimal detectable vessel diameter of 0.3 $\mathrm{mm}$ reflects more than $99.5 \%$ of the total collateral blood flow situation, for both day 7 and day 21. The additional vessels observed with a minimal detectable vessel diameter of $0.1 \mathrm{~mm}$ instead of $0.3 \mathrm{~mm}$ reflect less than $1 \%$ of the total collateral blood flow. 


\section{Discussion}

The results of this study demonstrate the potential of contrast-enhanced MRA for serial and non-invasive evaluation of early collateral vessel growth with sub-millimeter vessel diameters.

The visualization of collateral vessel growth by serial contrast-enhanced MRA in a rabbit femoral artery ligation model in correlation with XRA, was performed with use of a commercially available clinical 1.5 Tesla MRI system and a clinically approved MR contrast agent. Earlier studies ${ }^{13}$ reported the visualization of collateral vessels in a femoral artery ligation model with use of a dedicated animal MR system. Studies in patients ${ }^{14}$ "documented collateral artery growth by MRA in critical limb ischemia 3 and 9 months after vascular endothelial growth factor gene therapy. In the patient study MRA was performed with a lower spatial resolution than in the study presented here. Our use of a higher spatial resolution results in the depiction of smalier diameter vessels, which might make MRA useful for evaluating the early responses (e.g., weeks) to treatments, for example as used in the patient study ${ }^{14}$, before longer time scale changles (e.g., months) become apparent.

That the collateral vessels visualized here by MRA represent those collateral vessels that account for nearly $100 \%$ of the total collateral blood flow, was derived by indirect computationall flow analysis and with earlier results from a phantom study ${ }^{11}$ that minimal detectable vessel diameter by MRA was $0.3 \mathrm{~mm}$. It is noted that the applied theoretical flow model was not validated by flow measurements, but served only to roughly illustrate (not to accurately quantify) that the relative contributions of additionally observed vessels by XRA $(\sim 0.1 \mathrm{~mm})$ to the muscle blood flow is negligible compared to larger vessels ( $0.3 \mathrm{~mm}$ ) as observed by both XRA and MRA. Put in other words, one $0.3 \mathrm{~mm}$ diameter vessel mediates $81\left(=3^{4}\right)$ times more flow than one $0.1 \mathrm{~mm}$ diameter vessel according to the laminar flow assumptions. Future flow measurements of this ligation model, combined with histology to determine the vessel size distribution, could accurately quantify the progression of flow over time and validate the used computational model. Hence, XRA and MRA measurements, as performed here, not only provided morphologic information concerning growth of collateral blood vessels, but also give a sense of the functional restoration of blood flow after femoral artery ligation. Further work is necessary to relate this arbitrary sense of blood flow with quantitative flow or tissue perfusion measurements.

Differences in collateral vessels scored on XRA and on MRA images were largely related to inherent differences between these two imaging modalities. Foremost, a limitation of MRA was its sub-optimal spatial resolution. XRA inherently has a higher spatial resolution than MRA, which best explains the higher numbers of collateral vessels visualized with XRA than with MRA, particularly at days 7 and 21 . Furthermore, MRA is based on a relatively long measurement that requires a relatively slow intravenous contrast agent injection ( $1 \mathrm{~mL} / 30 \mathrm{~s})$ compared to the fast (12 frames per second) imaging with XRA that consequently requires fast (selective) intra-arterial injection $(5 \mathrm{~mL} / \mathrm{s}$ ) of contrast agent. The slow intravenous contrast agent injection with MRA might have resulted in a more physiologic distribution of contrast agent than for XRA. Physiologic flow distribution of the MR contrast agent supports the notion that collateral vessels visualized on MRA reflect collaterals that contribute most to recovery of blood flow to the distal portion of the limb.

Venous enhancement was encountered only with the relatively long MRA measurement, and not with XRA. As explained in the Methods section, venous enhancement was one 
reason why the MRA data were scored on three-dimensional rotational projections in order to discern collateral arteries from enhancing veins. These veins could be well identified on the three-dimensional projections, because of their connection to the main venular system. Venous enhancement on MRA might have interfered with the results; however, because subjectwely, more venous enhancement was seen in the control limb than in the ligated limb, and more venous enhancement was seen at day 21 than at day $0 *$ and day 7 , within the control limb. Possible relevance of differences in venous enhancement on MRA in the evaluation of patients with peripheral ischemia ${ }^{15}$ is uncertain, however:

Three-dimensional analysis of the MRA data allowed a more critical identification of the stem, mid-zone and re-entry zone of each collateral artery (according to the Longland criteria ${ }^{10}$ ) than on two-dimensional projections (i.e., non-continuous vessels were not scored). Another limitation was that three-dimensional side-by-side comparisons could not be performed, as our $X$-ray system provided projections only. Three-dimensional XRA, which was not available at our site, could enable side-by-side matching of the identified collateral vessels.

It was established previously ${ }^{1}$ that spontaneous collateral growth in these rabbit models result in nearly complete recovery of blood flow at rest, three weeks after femoral artery ligation. The number of collaterals on day 21 as reported in this study is consistent with previous studies that used post-mortem angiography. However, some studies report up to three-fold higher numbers of collateral vessels 7 days after femoral artery ligation ${ }^{2}$. A proposed mechanism for such a "peak" at 7 days is that a few large collateral vessels grow selectively to the disadvantage of numerous small ones that regress again (i.e., pruning) ${ }^{2}$. These numerous small collaterals at day 7 were not observed in our study, presumably because post-mortem XRA provides images superior in quality over in vivo XRA and MRA techniques used in this study. Critical evaluation of the data presented in Figure 4, on which the association calculations were based, suggests that XRA and MRA scores on days 7 and 21 (i.e., ignoring day $0+$ scores) were not positively associated. On the one hand a parallel increase in detectable collaterals is expected for XRA and MRA between day 7 and 21, but on the other hand the pruning effect might counteract the increase in the number (and variance) of large collaterals (i.e., the MRA score). However, the number of data points (i.e., animals) is too low to objectify this. Further work is necessary to evaluate whether higher (isotropic) spatial resolutions can be achieved with MRA, without compromising volume coverage or temporal resolution, to overcome the differences with XRA encountered in this study. Future studies of neovascularization would likely benefit from visualizing arteries as small as possible, particularly to investigate the earliest stages of collaterall artery progression and regression over time.

Development of dedicated MR receiver coils and use of higher magnetic field (e.g., animal) MR units might further improve visualization of collateral vessel growth by MRA. Intravascular (i.e., blood pool) T1-shortening MR contrast agents might allow prolonged acquisition times, and further improvements in spatial resolution ${ }^{16,17}$. In addition, developments such as venous compression and parallel imaging techniques might prove to be useful for suppressing venous enhancement. In our study, however "venous compression would interfere with the disease model, and parallel-imaging techniques that we evaluated in a pilot study (i.e., SENSE factors greater than 1.0) (data not shown) resulted in images with lower signal-to-noise ratios that obscured many (small) collateral vessels. 
Conclusion

Serial contrast-enhanced MR angiography non-invasively visualizes sub-mitlimeter collateral vessels that are responsible for blood flow recovery in a rabbit femoral artery ligation model. Monitoring collateral vessel development is important as it may become clinically helpful in both coronary and peripheral vascular disease, which would allow non-invasive assessment of collateral arteries and how they respond to treatments that stimulate their growth. 


\section{References}

1. Hershey JC, Basikin EP, Glass JD, Hartman HA, Gilberto DB, Rogers IT, Cook JJ. Revascularization in the rabbit hindlimb: dissociation belween capillary sprouting and anteriogenesis. Cardiovasc Res. 2001:49:618-25.

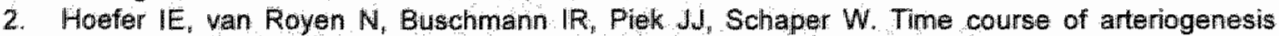
following femoral artery occlusion in the rabbit Cardiovasic Res. 2001;49:609-17.

3. Cao $R_{s}$ Brakenhielm $E$, Pawliuk $R$, Wariaro $D$, Post $M J$, Wahlberg $E$, Leboulch $P$, Cao $Y$. Angiogenic synergism, vascular stability and improvement of hind-limb ischemia by a combination of PDGF-BB and FGF-2. Nat Med. 2003;9:604-13.

4. Carmeliet $P$. Mechanisms of angiogeriesis and arteriogenesis. Nat Med. 2000;6:389-95.

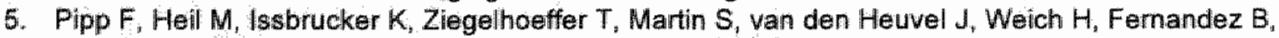
Golomb 0 , Carmeliet P, Schaper W. Clauss M. VEGFR-1-selective VEGF homologue PIGF is arteriogenic: evidence for a monocyte-mediated mechanism. Circ Res. 2003:92:378-85.

6. Buschmann I, Heil M, Jost M, Schaper W. Influence of inflammatory cytokines on arteriogenesis. Microcirculation. 2003:10:371-9.

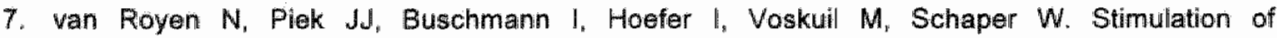
arteriogenesis; a new concept for the treatment of arterial occlusiwe disease. Cardiovasc Res. $2001: 49: 543-53$

B. Buschmann IR, Voskuil M, van Royen $N$, Hoefer IE, Scheffler $K$, Grundmann $S$, Hennig J, Schaper $W_{*}$ Bode $C$. Piek JJ. Invasive and non-invasive evaluation of spontaneous arteriogenesis in a novel porcine model for peripheral arterial obstructive disease. Atherosclerosis. 2003:167:3343.

9. Guyton AC, Hall JE. Overview of the Circulation; Mecial Physics of Pressure "Flow, and Resistance. In: Wonsiewicz MJ ed. Textbook of Medical Physiology. 10 ed. Philadelphia: W.B. Saunders Company; 2000:141-153.

10. Longland CJ. The collateral circulation of the limb; Arris and Gale lecture delivered at the Royal College of Surgeons of England on 4th February, 1953. Ann R Coll Surg Engl. 1953;13:161-76.

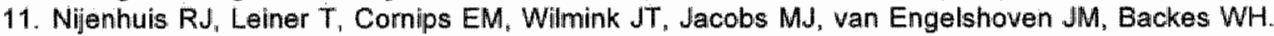
Spinal cord feeding arteries at MR angiography for thoracoscopic spinal surgery: feasibility study and implications for surgical approach. Radiology. 2004;233:541-7.

12. Cohen J. Weighted kappa: nominal scale agreement with provision for scaled disagreement or partial credit. Psycholl Bull. 1968;70:213-230.

13. Wagner $S$. Helisch $A$, Zlegellhoeffer $T$, Bachmann $G$, Schaper W. Magnetic resonance angiography of collateral vessels in a murine femoral artery ligation model. NMR Biomed. $2004 ; 17: 21-7$.

14. Baumgartner I, Thoeny $\mathrm{HC}_{\mathrm{s}}$ Kummer O, Roefke $\mathrm{C}$, Skjelsvik $\mathrm{C}_{\mathrm{s}}$ Boesch $\mathrm{C}$, Kreis R. Leg Ischemia: Assessment with MR Angiography and Spectroscopy. Radiology. 2005;234:833-41.

15. Wang $Y$, Chen $C Z$, Chabra $S G$, Winchester PA, Khilnani NM, Watts $R$, Bush HL, Jr., Craig Kent $K_{1}$ Prince MR. Bolus arterial-venous transit in the lower extremity and venous contamination in bolus chase three-dimensional magnetic resonance angiography. Invest Radiol. 2002;37:456-63.

16. Kobayashi $H$, Kawamoto $S$, Saga $T$, Sato $N$, Hiraga $A$, Konishi $J$, Togashi K, Brechbiel MW. Micro-MR angiography of normal and intratumoral vessels in mice using dedicated intravascular MR contrast agents with high generation of polyamidoamine dendrimer core: reference to pharmacokinetic properties of dendrimer-based MR contrast agents. J Magn Reson Imaging. $2001: 14: 705-13$.

17. Fink $C_{n}$ Kiessling $F$, Bock M, Lichy MP. Misselwitz $B$, Peschke $P$, Fusenig NE Grobholz $R_{8}$ Delorme $S$. High-resolution three-dimensional MR angiography of rodent tumars: morphologic characterization of intratumoral vasculature. J Magn Reson Imaging. 2003;18:59-65. 


\title{
Chapter 8
}

\section{Evaluation of Gd(III)DTPA-terminated poly(propylene imine) dendrimers as contrast agents for MR imaging}

NMR in Biomedicine, 2005 provisionally accepted

\author{
Sander Langereis, Quido G. de Lussanet, Marcel H. P. van Genderen, \\ E. (Bent) W. Meijer, Regina G. H. Berts-Tan, Arjan W. Griffioen, \\ Jos M. A. van Engelshoven, Walter H. Backes.
}

\section{ABSTRACT}

\section{PURPOSE AND METHODS}

Different generations of Gd(III)DTPA-terminated poly(propylene imine) dendrimers (G1 ( $n=4$ Gd(III) atoms per molecule), $\mathbf{G 3}(n=16)$ and $\mathbf{G 5}(n=64))$ and reference Gd(III)DTPA complex $(\mathbf{G} 0(n=1))$ were characterized in terms of $(i)$ longitudinal $\left(r_{1}\right)$ and transverse $\left(r_{2}\right)$ relaxivities in mouse blood plasma, (ii) concentration detection limits in vitro, and (iii) in vivo contrast-enhanced MR imaging (CE-MRI) in mice at $1.5 \mathrm{~T}$. Serial and dynamic CE-MRI were performed to monitor the distribution of MRI contrast agent in the heart, arteries, renal system, liver, spleen, bladder, and tumor periphery.

\section{RESULTS}

The relaxivities non-linearly increased with molecular weight (for $G_{0}$ ionic $r_{1}=8.1 \mathrm{~mm}^{-1} \mathrm{~s}^{-}$ 1 and ionic $r_{2}=8.6 \mathrm{~mm}^{-1} \mathrm{~s}^{-1}$ to $\mathbf{G 5} 19.3$ and 25.0 , respectively). The minimal detectable dendrimer concentration was more than two orders of magnitude lower for $\mathbf{G 5}\left(8.1 \times 10^{-}\right.$ $\left.{ }^{8} \mathrm{M}\right)$ than for $\mathrm{GO}\left(3.1 \times 10^{-5} \mathrm{M}\right)$. Sub-millimeter-sized blood vessels were well visualized with serial CE-MRI with each contrast agent. Dynamic CE-MRI showed timely renal clearance for all contrast agents, but a stronger and a prolonged blood signal enhancement for the higher generations of the dendritic contrast agent. Moreover, G0 and G1 showed a rapid tumor wash-in and wash-out, whereas G3 and G5 displayed a more gradual and prolonged tumor wash-in.

\section{CONCLUSION}

In conclusion, both $\mathbf{G 0}$ and dendritic contrast agents $\mathbf{G 1}, \mathbf{G 3}$ and $\mathbf{G 5}$ are weil suited for non-tissue specific MRI of sub-millimeter-sized blood vessels and evaluating tumor microcirculatory characteristics in mice. Higher generations of dendritic contrast agents display lower concentration detection limits, which suggest their future use for molecular imaging. 


\section{Introduction}

Low molecular weight (MW) contrast agents based on chelates of gadolinium(lil) (Gd(III)), are currently the most widely applied contrast agents for general clinical MR imaging. These MRI contrast agents are appreciated for their predominant positive signal enhancement in $T_{4}$-weighted MR imaging, non-specific (organ-) tissue distribution, and particular high safety profile. ${ }^{\text {t.3 }}$

For some imaging purposes, however, the properties of low MW contrast agents are sub-optimal. Firstly, low MW contrast agents rapidly diffuse from the blood space to the interstitial space, even in healthy vessels. This high relative permeability prevents their effective use for differentiating between benign lesions (normal vessels) and malignant tumors (hyper-permeable vessels) with contrast-enhanced MR imaging (CE-MRI) in oncology, while such differentiation is possible with the use of high MW contrast agents. Secondly, the rapid extravasation of low MW contrast agents causes a rapid decrease of the concentration of contrast agent in the blood. This is unfavorable for MR angiography purposes, because it will result in a lower signal enhancement in the blood, but also in a higher background tissue signal. Such increases in tissue background signal may hamper the spatiall detection limits, particularly for MR angiography of small blood vessels. Contrary to low MW contrast agents, high MW contrast agents have prolonged high blood concentrations with little background tissue enhancement. Therefore high MW weight contrast agents are commonly referred to as blood pool agents, and low MW contrast agents are referred to as extravascular agents. ${ }^{2}$

Thirdly. How MW weight contrast agents, such as the Gd(III) complex of diethylenetriaminepentaacetic acid (DTPA), contain only one Gd(III) ion per molecule. Consequently, the relatively low signal enhancing effect (or longitudinal relaxivity $r_{1}$, $m \mathrm{~m}^{-1} \mathrm{~s}^{-1}$ ) per molecule requires that relatively high concentrations (i.e. high doses or injection rates) of contrast agent must be administered to obtain a sufficiently high local concentration of Gd(III) that can be delineated from surrounding tissue.

A straightforward approach for increasing the local concentration of $\mathrm{Gd}(I I I)$ is to attach multiple $\mathrm{Gd}(\mathrm{III})$ chelates to a macromolecular scaffold. An additional advantage of attaching $\mathrm{Gd}(\mathrm{III})$ based complexes to high MW structures, either covalently or noncovalently, ${ }^{5-10}$ is that the $r_{1}$ per $\mathrm{Gd}$ (III) (i.e. the ionic relaxivity) increases due to the lower molecular tumbiling rate of the Gd(III) compllex."2

Several studies have shown that dendrimers are excellent macromolecular scaffolds to attach covalently multiple $\mathrm{Gd}(\mathrm{III})$ complexes. ${ }^{11-16}$ These highly branched architectures have a well-defined molecular weight and a precise number of end groups, which reside in virtually identical environments. ${ }^{17,48}$ Wiener ef al. ${ }^{11}$ and Kobayashi ef al. ${ }^{16}$ reported on the conjugation Gd(III)DTPA moieties to different generations of the poly(amidoamine) (PAMAM) and poly(propylene imine) (PPl) dendrimers, employing isothiocyanateactivated $p$-nitrophenylalanime-based DTPA chelates. These dendritic MRI contrast agents were shown to be useful for in vivo angiography, lymphography, evaluation of contrast agent distribution and clearance, and (liver) organ-specific imaging. ${ }^{16,19-22}$ Moreover, it was demonstrated that the ionic $r_{1}$ almost linearly increase with molecular weight upon going to higher generations of the dendritic contrast agents.

Recently, a different series of well-defined Gd(III)DTPA-terminated poly(propylene imine) dendrimers was synthesized employing an isocyanate-activated lysine-based DTPA chelate (Figure 1). ${ }^{3}$ For these dendritic MRI contrast agents both the longitudinal $\left(r_{1}\right)$ and transverse $\left(r_{2}\right)$ relaxivities in citrate buffer were also shown to increase upon going to higher generations of the dendrimer. An important consideration in designing high MW 
Gd(III)-based MRI contrast agents involves their distribution and (kidney) clearance, as well as their blood protein or cellular interactions to avoid undesirable biodistribution and toxicity of free Gd(II). Non-covalent (blood) protein interactions are expected to affect the relaxivities. ${ }^{8,24,25}$ Currently, the $T_{1}$ and $T_{2}$ relaxivities in blood plasma and the in wivo imaging- and distribution properties are unknown for the Gd(II)DTPA-terminated poly(propylene imine) dendrimers that were synthesized using the isocyanate-activated lysine-based DTPA chelate. Therefore, the purpose of this study was to evaluate different generations of these dendritic contrast agents, ranging from reference complex Go $(0.7 \mathrm{kDa})$ up to $\mathbf{G 5}(51.2 \mathrm{kDa})$, for which the MW should just be low enough for kidney clearance. These MRI contrast agents were evaluated and compared in terms of (i) $T_{1}$ and $T_{2}$ relaxivities in mouse blood plasma, (ii) relative signal changes and concentration detection limits in vitro, and (iii) distribution and clearance in mice, using common $T_{1}$-weighted MR imaging protocols at $1.5 \mathrm{~T}$.
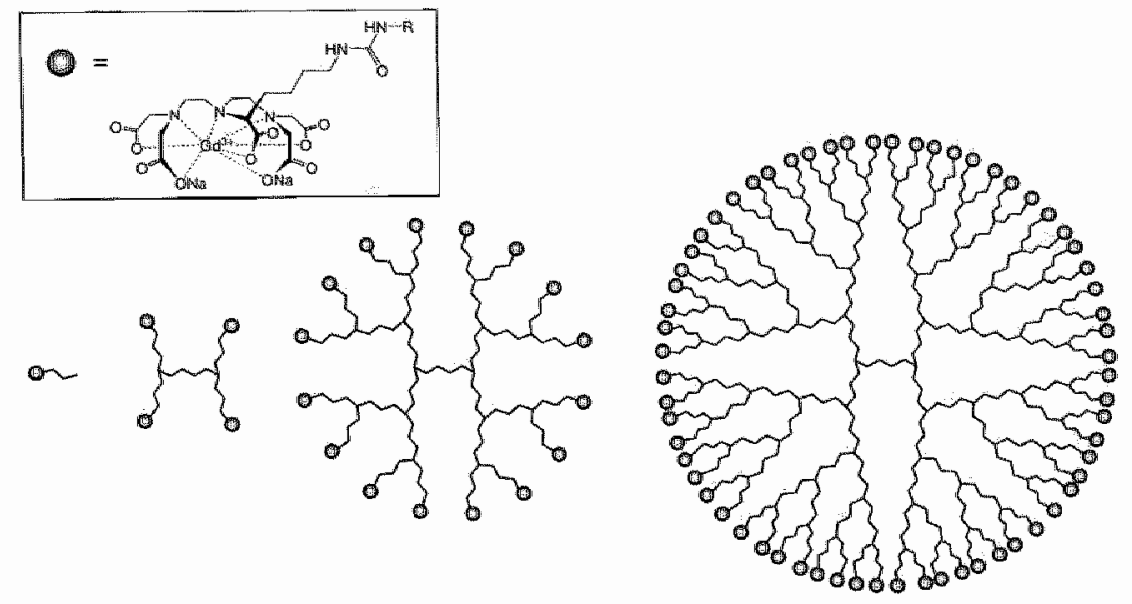

$G 0(n=1) \quad G 1(n=4)$

$\cos (n=16)$

GS $(n=6.4)$

Figure 1. Reference $G d(I I 1)$ complex ( $G 0(n=1)$, and different generations of $\operatorname{Od}(I I) D T P A=$ terminated poly(propylene imine) dendrimers ( $G 1(n=4), G 3(n=16), G 5(n=64)$ ). $G$ represents the generation of the dendrimer and $n$ represents the theoretical number of Gd(III)DTPA complexes attached to a single dendrimer.

\section{Materials and Methods}

Symthesis of dendritic MRI contrast agents

Different generations of the poly(propylene imine) dendrimers were obtained from SyMO-Chem BV (Eindhoven. The Netherlands). The polydispersity of the poly(propylene imine) dendrimers has been measured with electrospray mass spectrometry and it was found to be less than 1.003 for all dendrimers. ${ }^{26}$ The primary amines of the first, third and fifth generation of the poly(propylene imine) dendrimers (DAB-dendr- $\left.\left(\mathrm{NH}_{2}\right)_{n}\right)$ (with respectively 4,16 , and 64 end groups $(n)^{17}$ were reacted with an isocyanate-activated lysine-based DTPA pentaester in dichloromethane (DCM). ${ }^{23}$ Structural characterization 
with 'H-NMR, ${ }^{19}$ C-NMR, and IR spectroscopy and ESI-QTOF spectrometry showed complete modification of the primary amines of the dendrimers. ${ }^{23}$ Subsequently, the tertbutyl ester-protected DTPA-terminated poly(propylene imine) dendrimers were reacted with trifluoroacetic acid in DCM, dialyzed and complexated with $\mathrm{Gd}(11 \mathrm{ll}) \mathrm{Cl}_{3}$, to create Gd(II)DTPA-terminated dendrimers (G1 $(n=4), \mathbf{G} 3(n=16)$, and $\mathbf{G} 5(n=64)$ as shown in Figure $1^{23}$ Reference Gd(II)DTPA-based complex (GO $(n=1)$ was prepared in a similar fashion, starting from propylamine and the isocyanate-activated DTPA synthon. The theoretical molecular weights of reference Gd(III)DTPA complex (GO) and the different generations dendritic MRI contrast agents (G1, G3 and G5) are listed Table 1.

\section{In vitro MR imaging}

MR imaging was performed on a $1.5 \mathrm{~T}$ system (Philips Medical Systems, Best, The Netherlands) with a maximum gradient strength of $30 \mathrm{mT} / \mathrm{m}$ and rise time of $200 \mu \mathrm{s}$.

\section{Relaxivities in mouse plasma}

For the relaxivity measurements, different concentrations of Gd(III) for Gd(III)DTPA, G0, G1, G3, and G5 (range 0.05-1.0 mM) were prepared in mouse blood plasma (Harlan Sera-Lab, UK) with sodium citrate as anti-coagulant. Gd(III) concentrations used for the relaxivity measurements were determined by means of Inductively Coupled Plasma Atomic Emission Spectroscopy (ICP-AES). The MR measurements were performed using a circular surface coil (inner diameter, $8 \mathrm{~cm}$ ). Localized "H NMR spectra were recorded by using Point Resolved Spectroscopy (PRESS) ${ }^{27}$ with 16 acquisitions and 16 phase-encoding steps (voxel dimensions $10 \times 10 \times 10 \mathrm{~mm}$ ). Longitudinal relaxation times ( $T_{1}$ in $\mathrm{ms}$ ) were determined with an irversion recovery pulse-sequence using 10 different inversion times ranging from $9 \mathrm{~ms}$ to $2900 \mathrm{~ms}_{\text {; }}$ the repetition times (TR) were at least 5 times longer than the estimated $T_{1}$ of each sample, and the TE was set at $40 \mathrm{~ms}$. Transwerse relaxation times ( $T_{2}$ in $\mathrm{ms}$ ) were determined by varying the PRESS echo time ranging from 30 to $700 \mathrm{~ms}$ (TR $=1500 \mathrm{~ms}$, number of signal averages (NSA) 10).

\section{Signal enhancement}

Solutions of the MRI contrast agents in mouse blood plasma were prepared with a $\mathrm{Gd}(\mathrm{III})$ concentration in the range of $5.0 \times 10^{-7}$ to $1.0 \times 10^{-3} \mathrm{M}$, in vials with an inner diameter of $6 \mathrm{~mm}$. All vials were measured simultaneously by using a quadrature volume coil ( $k$ nee coil; inner diameter, $18 \mathrm{~cm}$ ). $T_{1}$-weighted MR measurements were performed (three-dimensional fast field echo (3D FFE); TR/TE 14/6 ms; flip angle (FA) $30^{\circ}$; NSA 2) with 30 coronal sections, $1.0 \mathrm{~mm}$ thick and subsequently interpolated to $0.5 \mathrm{~mm}$ thick sections during reconstruction. Matrix dimensions were $256 \times 256$, with a field of view of $168 \times 134 \mathrm{~mm}$, and reconstructed voxel sizes were $0.65 \times 0.52 \times 0.5 \mathrm{~mm}$. The average signal intensity ( \pm one standard deviation (SD)) was measured in each vial by selecting 60 ( \pm 5 ) central voxels in circular regions of interest in the central section thus avoiding Gibbs ringing artefacts. The relative signal change, defined as $\left(S-S_{0}\right) / S_{0}$ in which $S$ represents the MRI signal at a specified concentration and $S_{0}$ the MRI signal at concentration zero, was then calculated. 


\section{Animal Model}

The ethical review committee of our institution (Maastricht University Hospital, Maastricht, The Nethertands) approved the animal studies. The maintenance and care of the experimental animals was in compliance with the guidelines set by the institutional animal care committee, accredited by the National Department of Health. Fout healthy male mice (C57 black 6. Charles River, Maastricht, The Netherlands) were used for serial CE-MR measurements with G0, G1, G3, and G5 (dose 0.03 mmol Gd(ll)/kg body weight). Twelve male nude mice (Swiss nu/nu, Charles River, Maastricht) (age 9 weeks) received an hiection of $10^{\circ}$ human colon carcinoma cells (LS 174T) subcutaneously in their left flank. On day 16 after tumor cell injection mice tumors (average ( \pm SD) volume $153 \pm 104 \mathrm{~mm}^{3}$ ) were used for dynamic CE-MRl. The mice were anaesthetized with subcutaneous injection of $100 \mathrm{mg}$ of ketamine (Nimatek" Eurovet, Bladel. The Netherlands) per kilogram of body weight and $10 \mathrm{mg} / \mathrm{kg}$ of xylazine $\mathrm{HCl}$ (Sedamun. Eurovet).

The mice were randomly assigned to reference Gd(III) complex or one of the dendritic MRI contrast agents that were injected (approximate injection rate, $2 \mu \mathrm{L} / \mathrm{s}$ ) into the tail vein and flushed with $15 \mu \mathrm{L}$ of normal saline (NaCl $0.9 \%$ Injection Fluid, Braun, Melsungen, Germany) (2 $\mu / \mathrm{L}$ ). MR imaging was performed using a small circular surface coil (inner diameter, $5 \mathrm{~cm}$ ). Warm water bags were placed near the mouse to keep the local temperature in the MR imaging unit bore near $28{ }^{\circ} \mathrm{C}$. After MR imaging the mice, while stifl under anesthesia, were sacrificed by means of cervical dislocation.

\section{Serial CE-MRI}

The serial CE-MRI protocol included 6 MR acquisitions of 25 seconds each (one precontrast 'mask' acquisition, and five contrast-enhanced acquisitions were timed at $8 \mathrm{~s}$, $30 \mathrm{~s}, 1 \mathrm{~min}, 3 \mathrm{~min}$, and $8 \mathrm{~min}$ after the start of contrast agent injection) (3D FFE; TR/TE $12 / 3.2 \mathrm{~ms}$; $\mathrm{FA} 30^{\circ}$ ) with 30 coronal, $1.6 \mathrm{~mm}$ thick (interpolated to $0.8 \mathrm{~mm}$ ) slices. Matrix dimensions were $224 \times 224$, with a field of view (FOV) of $90 \times 90 \mathrm{~mm}$, and reconstructed voxel sizes were $0.4 \times 0.4 \times 0.8 \mathrm{~mm}$. Images were evaluated on maximum intensity projections (anterior posterior direction) of subtraction images (i.e. each contrastenhanced image data set minus the mask).

\section{Dynamic CE-MRI}

The dynamic CE-MRI protocol included a $T_{1}$-weighted dynamic contrast-enhanced series (3D FFE; TR/TE, $50 / 7 \mathrm{~ms}$; FA $35^{\circ}$ ) with 30 dynamic volume acquisitions of 39 seconds each, sixteen $4.0 \mathrm{~mm}$ thick axial sections (interpolated to $2.0 \mathrm{~mm}$ ). Matrix dimensions were $128 \times 128$, with a FOV of $64 \times 64 \mathrm{~mm}$, and reconstructed woxel sizes were $0.5 \times 0.5 \times 2.0 \mathrm{~mm}$. The MRI contrast agent was slowly injected ( $2 \mu \mathrm{L} / \mathrm{s})$ during the fifth dynamic volume acquisition. The average signal intensities (arbitrary units, $\mathrm{AU}$ ) prior to contrast agent injection (i.e. $\mathrm{S}_{0}$ ) were recorded by defining regions of interest for the blood in the aorta, the tumor rim (i.e., outer 1-2 mm margin of the tumor in the central section through the tumor), the renal collecting system, and the bladder. The tumor rim was selected because this area of the tumor is considered the leading edge of the tumor in which processes such as tumor angiogenesis are most prominent. ${ }^{2 E}$ The signal time course $\left(S_{1}\right)$ was averaged for the three mice for each of the contrast agents, and the relative signal over time was calculated $\left(S_{\mathbb{t}} / S_{0}\right)$. 


\section{Results}

\section{In vitro MR imaging}

\section{Relaxivities in mouse plasma}

The longitudinal $\left(r_{1}\right)$ and transwerse $\left(r_{2}\right)$ relaxivities for $G d(I I) D T P A$, reference $G d(I I)$ complex (G0), and different generations of the dendritic contrast agents (G1, G3 and G5) determined in mouse plasma at $1.5 \mathrm{~T}$, with good linear fits $\left(R^{2}>0.999\right)$ (Figure $2 \mathrm{a}$ and $2 b)$ are presented in Table 1 . The lonic relaxivities increased upon going to higher generations of the Gd(III)DTPA-based dendrimer, and with respect to Gd(III)DTPA and G0, such that the ionil $r_{1}$ and ionic $r_{2}$ for G5 $_{5}$ were at least four times higher than for the parent Gd(III)DTPA complex. In addition, the ratio $r_{2} / r_{4}$ increased upon going to higher generations of dendritic contrast agent.

Table 1. Longitudinal ( $r 1)$ and transverse ( $r 2)$ relaxivities of Gd(III)DTPA, the reference Gd(III)DTPA complex (GO) and different generations of the Gd(II)DTPA-terminated poly(propylene imine) dendrimers (G1, G3, and G5) in mouse plasma at $1.5 \mathrm{~T}$ and 20 ${ }^{\circ} \mathrm{C}$.

\begin{tabular}{cccccccc} 
& $\begin{array}{c}\text { Ionic } r_{1}^{8} \\
\left(\mathrm{mM}^{-1} \mathrm{~s}^{-1}\right)\end{array}$ & $\begin{array}{c}\text { Ionic } r_{2} \\
\left(\mathrm{mM}^{-1} \mathrm{~s}^{-1}\right)\end{array}$ & $\begin{array}{c}\text { Ratio } \\
r_{2} / r_{1}(-)\end{array}$ & $\begin{array}{c}\text { \#Gd } \\
\text { ions }\end{array}$ & $\begin{array}{c}\text { Mol. } r_{1} \\
\left(\mathrm{mM}^{-1} \mathrm{~s}^{-1}\right)\end{array}$ & $\begin{array}{c}\text { Mol. } r_{2}^{8} \\
\left(\mathrm{mM}^{-1} \mathrm{~s}^{-1}\right)\end{array}$ & $\begin{array}{c}\mathrm{MW}^{0} \\
(\mathrm{kDa})\end{array}$ \\
\hline GD(III)DTPA & $4.2 \pm 0.1$ & $4.5 \pm 0.3$ & 1.07 & 1 & 4.2 & 4.5 & 0.6 \\
G0 & $8.1 \pm 0.3$ & $8.6 \pm 0.2$ & 1.06 & 1 & 8.1 & 8.6 & 0.7 \\
G1 & $11.4 \pm 0.4$ & $12.0 \pm 0.5$ & 1.05 & 4 & 45.6 & 48.0 & 3.1 \\
G3 & $15.2 \pm 0.2$ & $17.9 \pm 0.8$ & 1.17 & 16 & 243 & 286 & 12.7 \\
G5 & $19.3 \pm 0.4$ & $25.0 \pm 0.6$ & 1.29 & 64 & 1235 & 1600 & 51.2 \\
\hline
\end{tabular}

\footnotetext{
The ionic relaxivity is defined as the relaxivity per $\mathrm{Gd}($ (III)

"The maximum molecular relaxivity $\left(\right.$ mol. $_{r, 2}$ ) is defined as the ionic relaxivity (ionic $r_{1,2}$ ) multiplied with the theoretical number of $\mathrm{Gd}(\mathrm{lll})$ ) moieties (in) attached to an single dendrimer

- Theoretical molecular weight (KDil)
}

\section{Signal enhancement}

The in witro $T_{1}$-weighted MR measurements of the different concentrations of the dendritic contrast agents and Gd(III)DTPA in mouse plasma showed that the higher generations $\mathbf{G 3}$ and $\mathbf{G 5}$ gave the best signal enhancement at low concentrations. The lower MW contrast agents Gd(III)DTPA, G0, and $\mathbf{G 1}$ gave the best visual signal enhancement at higher concentrations; concentrations at which $\mathbf{G} 3$ and $\mathbf{G 5}$ already showed $T_{2}{ }^{*}$ susceptibility effects (Figure 3 ). These $T_{2}{ }^{*}$ susceptibility effects caused a drop in signal enhancement at the highest concentrations for each contrast agent, and suggest an inverse relationship between generation of dendritic contrast agent and the concentration at which $T_{2}{ }^{*}$ effects depress signal enhancement. Figure $4 a$ shows the relative signal change versus the $G d(I I I)$ concentration, which reflects the differences in ionic relaxivity. Figure $4 \mathrm{~b}$ shows the rellative signal change versus the dendrimer concentration reflecting the relative molecular relaxivities. In correspondence with the literature value of $5 \times 10^{-5} \mathrm{M}$ for the lowest detectable concentration of Gd(III)DTPA ${ }^{3,29}$ a 
threshold relative signal change $\left(\left(S-S_{0}\right) / S_{0}\right)$ of 0.76 was used to derive the concentration limits for the dendritic contrast agents and the reference complex. The minimal detection limits for $\mathbf{G 0}$ to $\mathbf{G 5}$ appear along this threshold at decreasing concentrations of $\mathrm{Gd}(\mathrm{III}$ ) (see Table 2). In a similar manner, this analysis was carried out as a function of dendrimer concentration (Figure 4b). The minimal detection limits expressed in terms of dendrimer concentration are related by the number of end groups and are listed in Table 2. The minimal detectable dendrimer concentration was more than two orders of magnitude lower for $\mathrm{G5}\left(8.1 \times 10^{-8} \mathrm{M}\right)$ than for $\mathbf{G O}\left(3.1 \times 10^{-5} \mathrm{M}\right)$.

(a)

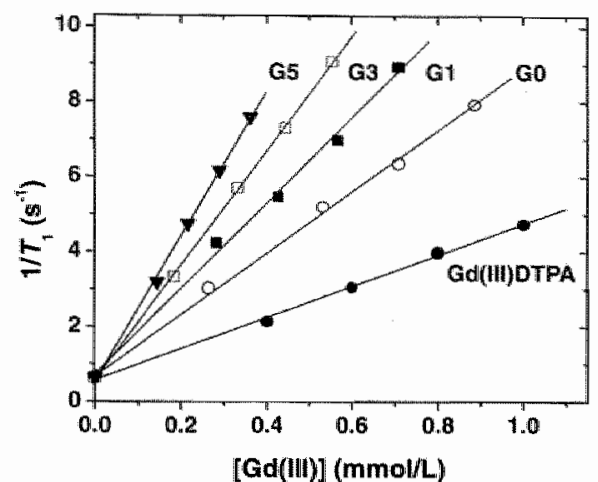

(b)

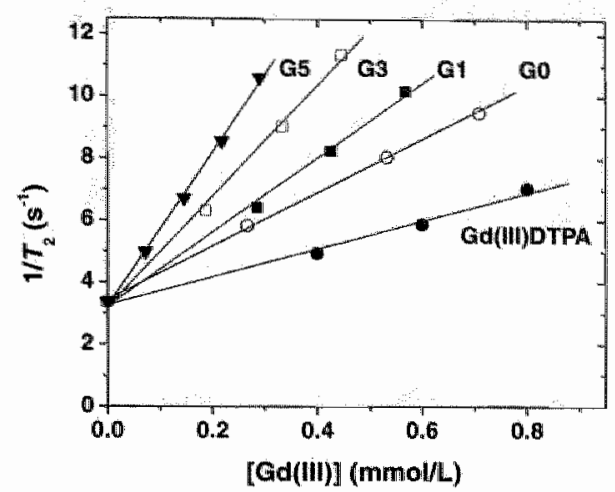

Figure 2. In vitro relaxivities of different generations of the Gd(III)DTPA-terminated poly(propylene imine) dendrimers in mouse plasma at $1.5 \mathrm{~T}$ and $20^{\circ} \mathrm{C}$. (a) Longitudinal relaxation rate $\left(4 / T_{1}\right)$ versus the concentration of $\mathrm{Gd}(\mathrm{III})$; (b) Transverse relaxation rate $\left(1 / T_{2}\right)$ versus the concentration of $\mathrm{Gd}(\mathrm{III})$.

Tablle 2. Limits of detectable concentrations for reference $\mathrm{Gd}(I I I)$ complex (GO) and different generation of the Gd(III)DTPA-terminated poly(propylene imine) dendrimers. The detection limit for Gd(III)DTPA was assumed to be $5.0 \times 10^{-5} \mathrm{~m}^{3}$

\begin{tabular}{cccc}
\hline & {$[\mathrm{Gd}(I I)](\mathrm{M})$} & \#Gd(iii) ions & [dendrimer] (M) \\
G0 & $3.1 \times 10^{-5}$ & 1 & $3.1 \times 10^{-5}$ \\
G1 & $1.9 \times 10^{-5}$ & 4 & $4.8 \times 10^{-8}$ \\
G3 & $1.5 \times 10^{-5}$ & 16 & $9.3 \times 10^{-7}$ \\
G5 & $5.2 \times 10^{-6}$ & 64 & $8.1 \times 10^{-8}$ \\
\hline
\end{tabular}




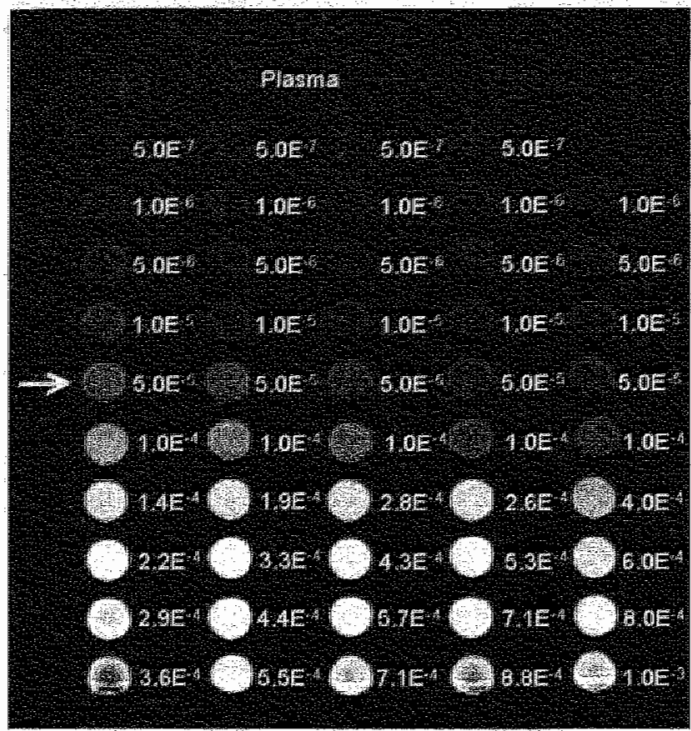

Figure 3. in vitro $T_{1}$-weighted $M R$ measurements of different concentrations of Gd(III) (M) of Gd(III)DTPA, reference Gd(II)DTPA complex (GO) and the dendritic contrast agents in mouse plasma visualizes that at a concentration of, for example, $50 \times 10^{-5} \mathrm{M}$ (row indicated by arrow) the signal increases upon going to higher generations of the dendritic contrast agent, which correspond with the findings in Table 2. The higher Gd(IIl) concentrations illustrate (i) a shift towards lower concentrations for the maximum signal enhancement with higher generations, and (ii) that concentrations at which $T_{2}{ }^{*}$ susceptibility effects are observed for the higher generations (G3 and $\mathrm{G5}$ ) are well below the concentrations of maximum signal enhancement for the

G5 G3 G1 G0 Gd-DTPA lower generations and Gd(III)DTPA. Note that the higher concentration tubes were positioned at the extreme of the phantorm, which is most distant from the magnet's iso center, and causes additional $T 2^{\text {* }}$ shortening (with signal intensity distortions) due to $\mathrm{B}_{0}$ inhomogeneities.

(a)

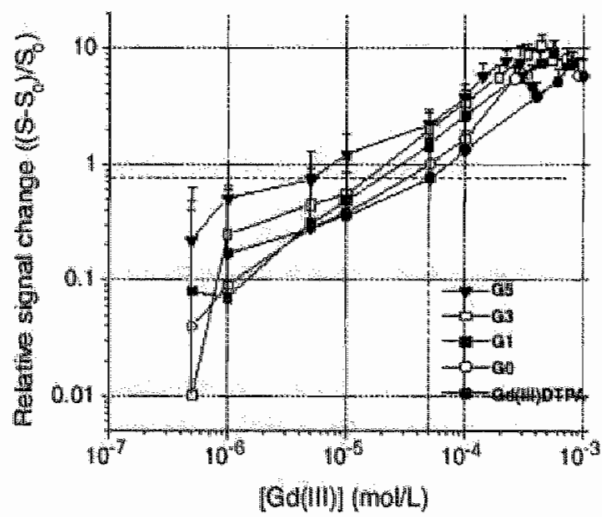

(b)

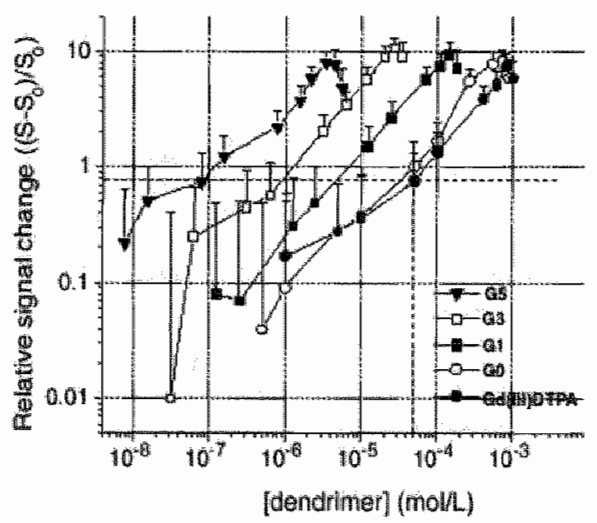

to $5.0 \times 10^{-5} \mathrm{M}$ (vertical broken line) giving rise to rellative signal change $\left(\left(S-S_{0}\right) / S_{0}\right)$ of at least 0.76 (horizontall broken line). (a) The log-log plot of the relative signal change versus the concentration of $\mathrm{Gd}(\mathrm{III})$; (b) The log-log plot of the relative signal change versus the concentration of dendritic contrast agent shows that the minimal detectable dendrimer concentration was more than two orders of magnitude lower for $\mathbf{G 5}\left(8.1 \times 10^{-8} \mathrm{M}\right)$ than for $\mathrm{Go}$ $\left(3.1 \times 10^{-6} \mathrm{M}\right)$. 


\section{Animal model}

\section{Serial CE-MRI}

The digital subtraction maximum intensity projections of the serial GE-MR measurements show comparable images of the cardiovascular system directly ( $8 \mathrm{~s}$ ) after contrast injection for each of the contrast agents, with comparable signal enhancement in the renal parenchyma (Figure 5). Thirty seconds after contrast agent injection observed differences are that the low MW contrast agents G0 and G1 show clearance through the renal collecting system, while the higher MW agents $\mathbf{G} 3$ and $\mathbf{G 5}$ contrast agents show some accumulation in liver and spleen. Eight minutes after contrast agent injection the contrast enhancement of the cardiovascular system had diminished, and all studied MR contrast agents were to some extent cleared by the renal system, which can be observed by the strong signal enhancement in the bladder (Figure 5).

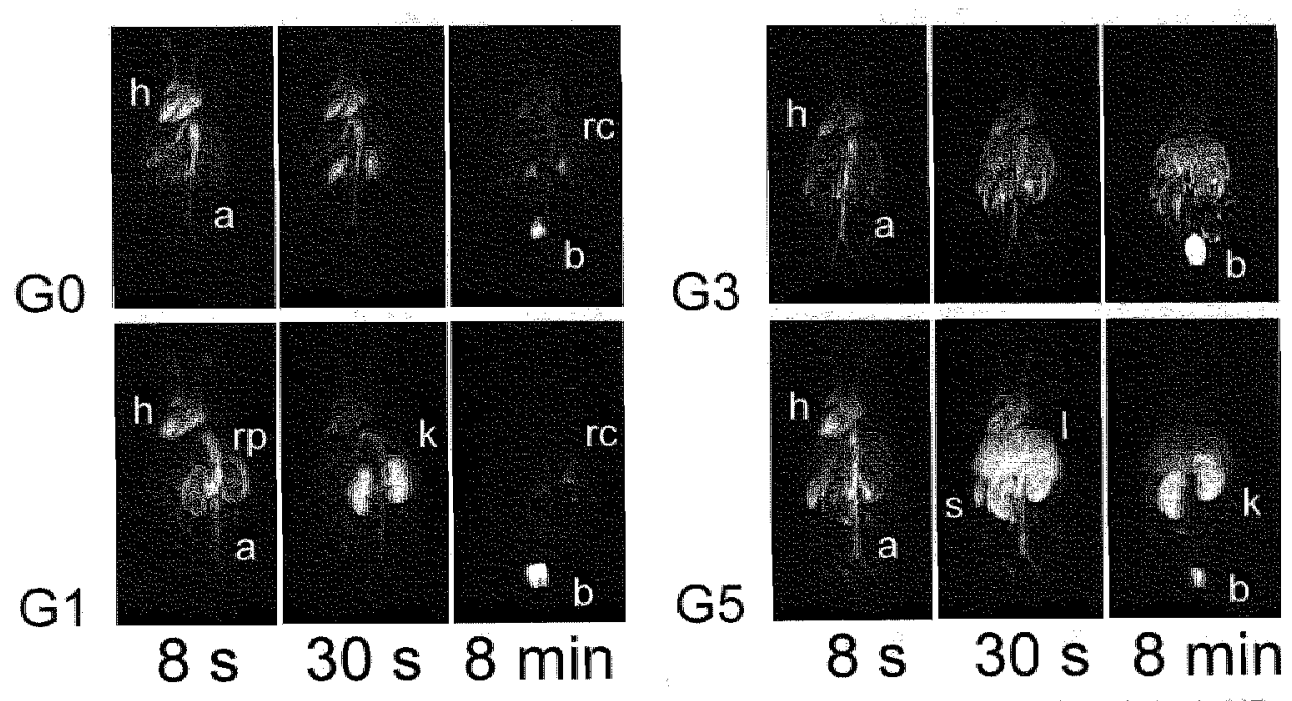

Figure 5. Maximum intensity projections of digitally subtracted contrast-enhanced whole body MR images show good visualization of the cardiovascular system (h- heart: a aorta) and the renal parenchyma (rp) directly after contrast agent injection $(t=8 \mathrm{~s}$ ), In time the lower generations appear to accumulate in the kidney $(\mathrm{k})$ while the higher generations appear to accumulate in the liver (I) and spleen (s). Eight minutes after MRI contrast agent injection the higher gienerations of the dendritic contrast agents accumulate in the renal collecting system (rc) of the kidney ( $k$ ) and to some extent in the bladder (b).

\section{Dynamic CE-MRI}

Dynamic CE-MR\| (semi-quantitatively) illustrates differences in circulation properties between the low and high MW weight contrast agents. Low MW contrast agents $G 0$ and G1 are cleared from the blood at a faster rate than the higher generations of the dendritic contrast agents (G3 and G5) (Figure 6a). The low MW agents showed a rapid tumor wash-in and wash-out, whereas $\mathbf{G . 3}$ and $\mathbf{G 5}$ showed a more gradual and prolonged tumor wash-in (Figure 6b). Reference Gd(III)DTPA complex and all generations of dendritic contrast agents were cleared through the kidneys as can be observed by the renal collecting system and bladder signal intensity curves, albeit that 
contrast agents clearance slowed as the molecular weight of the contrast agent increased (Figure $6 \mathrm{c}$ and $6 \mathrm{~d}$ ).

(a)

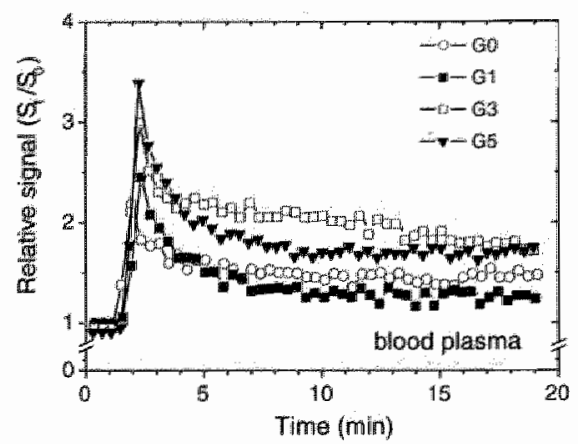

(c)

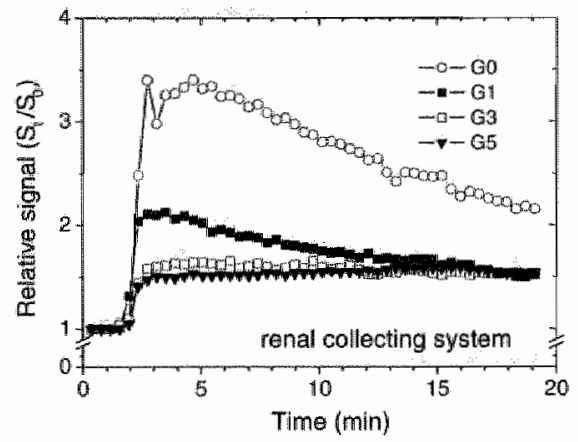

(b)

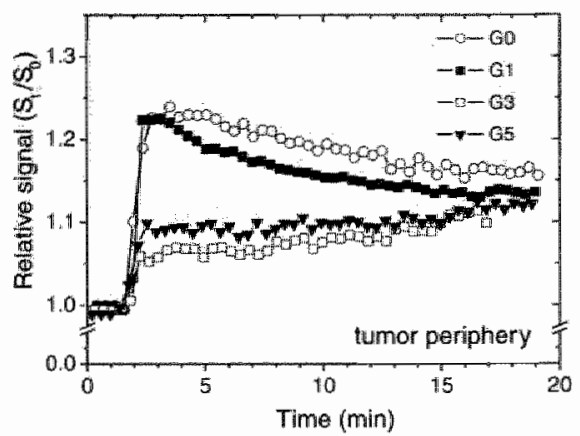

(d)

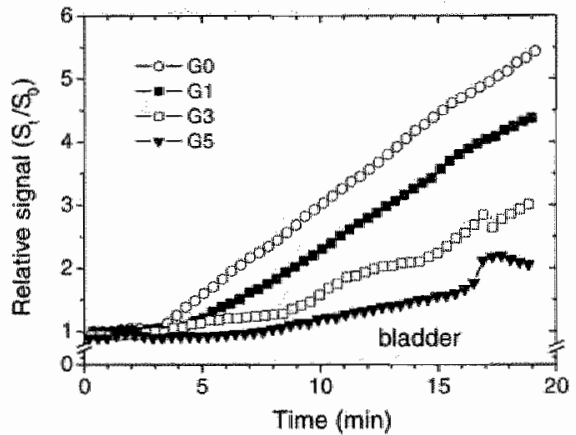

Figure 6. Relative signal over time measured with dynamic CE-MRI show the relatively stronger signal in the blood (a) for the higher generations of the dendritic MR contrast agents than the lower generations. Still all dendritic contrast agents show a typical bolus (strong signal increase) directly after contrast agent, a rapid distribution phase (fast signal decline) and later (approximately $t=5 \mathrm{~min}$ ) the clearance phase (slow signal decline). The relative signal intensity changes in the tumor periphery (b) with the lower generations contrast agents resemble the changes observed in the bload, whereas the higher generations show a gradual increase in signal intensity in time; (c) the renal collecting system and (d) bladder relative signal courses suggest an inverse relationship between the rate of renal clearance and molecular weight of the contrast

\section{Discussion}

This study showed that $T_{1}$ and $T_{2}$ relaxivities increase with higher generations of the Gd(III)DTPA-terminated poly(propyllene imine) dendrimers, causing the signal enhancement to increase and the minimal detectable concentration on MRI to reduce with higher generations. The dendritic contrast agents were well suited for serial CE-MRI of sub-millimeter-sized blood vessels and evaluating tumor microcirculatory characteristics by dynamic CE-MRI in mice. 
The $r_{1}$ and $r_{2}$ values of the different generations of the Gd(II)DTPA-terminated poly(propylene imine) dendrimers measured here in mouse blood plasma were good in agreement with those reported earlier by Langereis ef al. measured in either citrate buffer or water. ${ }^{23}$ This agreement between the $r_{1}$ results suggests that the dendritic MRI contrast agents had negligible interactions with blood plasma proteins, since noncovalent interactions between MR contrast agent and (blood-) proteins may result in (unpredictable) increases in ionic $r_{4}$ and changes in pharmacokinetic properties. ${ }^{2,8,24,25}$

Measuring both $r_{1}$ and $r_{2}$ illustrates their interrelationship. Accounting for $r_{2}$, even with $T_{1-}$ weighted imaging is important, because high $r_{2}$ values imply the possible occurrence of stronger $T_{2}$ and $T_{2}{ }^{*}$ effects. Direct $T_{2}{ }^{*}$ rellaxation measurements were not performed here because these would still not fully predict the $T_{2}$ " susceptibility effects in vivo. In theory. $T_{1}$-weighted images are not affected by $T_{2}^{*}$ effects when the echo time (TE) is infinitely short. In practice, TE is limited by the sampling time necessary to acquire one echo signall. This means that the shortest possible TE will increase going to larger acquisition matrices, which are required for high spatial resolution $T_{1}$-weighted imaging, resulting in $T_{2}{ }^{*}$ effects. For example, this was observed for the highest concentrations of contrast agents in the in vitro relaxation measurements. The susceptibility effects that might occur when larger acquisition matrices are used are counteracted by reduced intra-voxel dephasing for smaller voxels, which makes the prediction and quantification of $T_{2}{ }^{*}$ effects inaccurate. $9,12,30$ The finding that the highest generation dendritic MRI contrast agent (G5) gives the lowest detection limit would make G5 potentially the best candidate for future molecular imaging purposes (of the contrast agents evaluated in this study). In theory, the higher $T_{1}$ relaxivity of $\mathbf{G 5}$ makes it a better candidate for visualizing small blood vessels than the lower generations of the dendrimers. In addition, G5 has a longer blood circulation time compared to the lower generations dendritic contrast agents. A favorable characteristic of the low MW contrast agents is that relatively small $T_{2}{ }^{*}$ effects are observed with $T_{4}$-weighted imaging at high concentrations of contrast agents. This characteristic may benefit CE-MR imaging with fast bolus injection of large contrast agent dosages, for example for first-pass MR arteriography of large vessels. The rapid diffusing properties of low MW contrast agents may be used to their advantage, for example for evaluating certain microcirculation properties (i.e. microvessel flow instead of vessel wall permeability) by dynamic CE-MRI. ${ }^{31}$

CE-MR\| in mice suggests non-specific diffusion of the dendritic contrast agents into the tumor interstitial space. The observed differences in the rate of diffusion were likely related to the relative permeability through the wall of the blood vessel for the differently sized contrast agents. ${ }^{31}$ No evidence was found that the dendritic agents internalize into cells or have cellular interactions or matrix binding. The MRI protocol used here did not show the expected differences in visualization of small blood vessels between $\mathrm{GO}$ and G5 that one might expect based on the in vitro measurements. Each of the studied MRI contrast agents visualized small blood vessels such as the vessels in the neck (these were likely both the carotid artery and jugular vein) and hind-limbs, which typically have diameters of less than $0.4 \mathrm{~mm}$. The relatively high volume of coverage with high spatial resolution served the purpose of serial CE-MRI of the distribution of contrast agent. For imaging specific arterial systems this MRI protocol should be optimized.

\section{Conclusion}

Gd(II)DTPA-terminated poly(propylene imine) dendrimers 61, G3, and G5 show an increase of both the $T_{1}$ and $T_{2}$ relaxivities with increasing molecular weight. Relaxivity values, measured in blood plasma, are in good agreement with earlier measurements in 
citrate buffer which shows that these dendritic contrast agents have negligible interactions with blood plasma proteins. These dendritic contrast agents are well sulted for imaging of sub-millimeter-sized blood vessels and evaluating tumor microcirculatory characteristics in mice. Even the largest contrast agent presented with 64 gadolinum atoms was shown to be cleared by the kidneys. Dendritic contrast agents with increasing number of gadolinium atoms display higher signal enhancement and lower concentration detection limits. The lowest detectable concentration for the $\mathrm{G} 5$ dendrimer $(\sim 80 \mathrm{nM})$ was more than two orders of magnitude lower than for Gd-DTPA. Further research is required to demonstrate the efficacy of Gd(III)DTPA-functionalized poly(propylene imine) dendrimers as contrast agents for molecular imaging. 


\section{References}

1. Caravan, P.; Ellison, J. J.; McMurry, T. J.; Lauffer, R. B. Gadolinim(m) chelates as MRl contrast agents: structure, dynamics, and applications, Chem. Rev. 1999; 99:2293-2352.

2. Merbach, A. E.; Toth. E. The chemistry of contrast agents in medical magnetic resonance imaging; John Wiley \& Sons: New York, 2001

3. Jacques, V.; Desreux, J. F. New classes of MRI contrest agents, Top. Curr. Chem. 2002; 221: $123-164$.

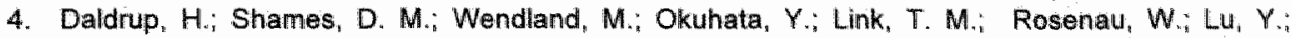
Brasch, R. C. Correlation of dynamic contrast-enhanced MR imaging with histologic tumor grade: comparison of macromolecular and small-molecular contrast media, Am. J. Roentganol. 1998; 171: $941-949$.

5. Lauffer, R. B.; Parmelee, D. J.; Dunham, S. U.; Ouellet, H. S.; Dolan, R. P.; Witte, S.; McMlurry, T. $J_{\text {; }}$ Walovitch, R. C. MS-325: albumin-targeted contrast agent for MR angiography, Radiology 1998; $207: 529-538$.

6. Wallace, R. A.; Haar, J. P., Jr.; Miller, D. B.; Woulfe, S. R.; Polta, J. A.; Gallen, K. P.; Hynes, M. R.; Adzamli, $K$. Synthesis and preliminary evaluation of MP-2269: a novil, nonaromatic smallmolecule blood-pool MR contrast agent, Magn. Reson. Med. 1998; 40: 733-739.

7. Aime, S.; Dastru, $W$ : Crich Simonetta, $G$; Gianolio, $E$; Mainero, $V$. Innovative magnetic resonance imaging diagnostic agents based on paramagnetic Gd(III) complexes, Biopolymers 2002; $66: 419-428$

8. Caravan, P; Cloutier, N. J; Greenfield, M. T; McDermid, S. A.; Dunham, S. U.; Bulte, $\downarrow$. W. M.; Amedio, J. C., Jr.; Looby, R. J.; Supkowski, R. M.; Horrocks, W. D., Jr.; MeMurry, T. J.; Lauffer, R. $B$. The interaction of MS-325 with human serum albumin and its effect on proton relaxation rates, J. Am. Chem. Soc 2002; 124:3152-3162.

9. Langereis, S.; Kooistra, H. A. T.; van Genderen, M. H. P.; Meljer, E. W. Probing the interaction of the biotin-avidin complex with the relaxivity of biotinylated Gd-DTPA, Org. Biomol. Chem. 2004; 2 : $1271-1273$.

10. Dirksen, A; Langereis, S; de Waal B. F. M.; van Genderen, M. H. P.; Hackeng, T. M.; Meijer, E. W. A supramolecular approach to multivalent target-specific MRI contrast agents for Angiogenesis, Chem. Commun. 2005; 22: 2811-2813.

11. Wiener, E. C.; Brechbiel, M. W.; Brothers, H.; Magin, R. L.; Gansow, O. A.; Tomalia, D. A.; Lauterbur, P. C. Dendrimer-based metal chelates: a new class of magnetic resonance imaging contrast agents, Magn. Reson. Med. 1994; 31: 1-8.

12. Wiener, E. C.; Auteri, F. P.; Chen, J. W.; Brechbiel, M. W; Gansow, O. A.; Schneider, D. S.; Belford, R. L.; Clarkson, R. B.; Lauterbur, P. C. molecular dynamics of ion-chelate complexes attached to starburst dendrimers, J. Am. Chem. Soc. 1996; 118: 7774-7782.

13. Tóth, E.; Pubanz, D." Vauthey, S.; Helm, L.; Merbach, A. E. High-pressure NMR kinetics. 72. The role of water exchange in attaining maximum relaxivities for dendirimeric MRI contrast agentsis Chem. Eur. J. 1996; 2: 1607-1615.

14. Fischer, $M_{\text {.; }}$ Vögtle, F. Dendrimers: from design to application - a progress report, Angew. Chem. Int. Ed. 1999; 38: 885-905.

15. Stiriba, S.-E.; Frey, H.; Haag, R. Dendritic polymers in biomedical applications: From potential to clinical use in diagnostics and therapy, Angew. Chem. Int. Ed. 2002; 41: 1329-1334.

16. Kobayashi, $H_{\text {; }}$ Kawamoto, S.; Jo, S.-K.; Bryant, H. L.. Jr.: Birechbiel, M. W. Star, R. A. Macromolecular MRI contrast agents with small dendrimers: pharmacokinetic differences between sizes and cores, Bioconjugate Chem. 2003; 14:388-394.

17. Bosman, A. W. Janssen, H. M.; Meijer, E. W. About dendrimers: structure, physical properties, and applications, Chem. Rov. 1999; 99: 1665-1688.

18. Grayson, S. M.; Frechet, J. J. M. Convergent dendrons and dendrimers: from synthesis to applications, Chem. Rev. 2001; 101: 3819-3868.

19. Kobayashi, $H_{-;}$Kawamoto $S_{*}$ Choyke, P. L; Sato, N.; Knopp, M. V." Star, R. A.; Waldmann, T. A.; Tagaya, $Y_{\text {; }}$ Brechbiel, M. W. Comparison of dendrimer-based macromolecular contrast agents for dynamic micro-magnetic resonance lymphangiography, Magn. Reson. Med. 2003; 50 : $758-766$. 


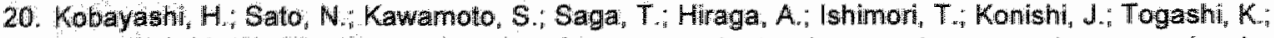
Brechibiel, M.W. 3D MR angiography of intratumoral vasculature using a novel macromolecular MR contrast agent, Magn. Reson. Med. 2001; 46: 579-585.

21. Kobayashi, H: Kawamoto, S.; Saga, T.; Sato, N.; Hiragia, A.: Ishimoni, T.; Akita, Y,; Mamede, M. H.; Konishi, J;; Togashi, K.; Brechbiel, M. W. Novel liver macromolecular MR contrast agent with a polypropylenimine diaminobutyl dendiumer core: comparison to the vascular MR contrast agent with the polyamidoamine dendrimer core, Magn. Reson: Med 2001, 46. 795-802.

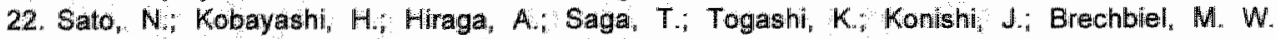
Pharmacokinetics and enhancement patterns of macromolecilar MR contrast agents with various sizes of polyamidoamine dendrimer cores, Magn. Reson. Med. 2001; 46: 1169-1173.

23. Langereis, $S$; de Lussanet, $Q . G_{\text {; }}$ wan Genderen, $M . H . P_{i}$ : Backes, $W$. $H_{\text {; }}$ Mejer, $E$. W. Multivalent contrast agents based on Gd(III)DTPA-terminated poly(propylene imine) dendrimers for magnetic resonance imaging. Macromolecules 2004; 37; 3084 -3091.

24. Hovland, $\mathbb{R} ;$, Aasen, A. J.; Klaveness, d. Preparation and in vitro evaluation of GdDOTA-(BOMl) 4 : a novel angiographic MRI contrast agent. Org. Biomol. Chem. 2003; 1: 1707-1710.

25. Aime $S$; Fasano $M$; Terreno, $E$. Lanthanide(III) chelates for NMR biomedical applications. Chem. Soc. Rev. 1998; 27: 19-29:

26. Hummelen, J. C.; Van Dongen, J. L. J.; Meijer, E. W. Electrospray mass spectrometry of poly(propylene imine) dendrimers - the issue of dendritic purity or polydispersity, Chem. Eur. $d$. 1997: 3: 1489-1493.

27. Bottomley, P. A. Selective volume method for performing localized NMR spectroscopy, U.S. Patent 44802281984.

28. Carmellet, $P$; jain, $R$. K. Angiogenesis in cancer and other diseases, Nature 2000; 407: 249-257.

29. Nunn, A. D.; Linder, K. E.; Tweedle, M. F. Can receptors be imaged with MRI agents?, Q. J. Nucl. Med. 1997; 41: 155-162.

30. Nicolle, G. M.; Toth, E.; Schmitt-Willich, H.; Raduchel, B.; Merbach, A. E. The impact of rigidity and water exchange on the relaxivity of a dendritic MRI contrast agent, Chem. Eur. J. 2002; 8: $1040-1048$.

31. de Lussanet, Q. G.; Langereis, S.; Berets-Tan, R. G. H.; van Genderen, M. H. P.; Griffioen, A. W.; van Engelshoven, J. M. A ; Backes; W. H. Dynamic contrast-enhanced MR imaging kinetic parameters and molecular weight of dendritic contrast agents in tumor angiogenesis in mice, Radiology 2005; 235: 65-72. 


\section{Chapter 9}

\section{General Discussion}

\section{General Discussion}

The work presented in this thesis underlines the potential of DCE-MRI for evaluating neo-vascularization in oncology, and introduces its use in peripheral arterial occlusive disease.

\section{Introduction}

The essence of DCE-MRI is that dynamic MR imaging data is combined with a pharmacokinetic model. Those who pioneered in DCE-MRI envisioned that a circulating MR contrast agent counts as a molecular tracer and that the rate of exchange of contrast agents between blood and tissue might therefore be quantifiable by the pharmacokinetic model.

Knowing that the exchange between blood and tissue occurs at the level of the microvasculature, the hypothesis was affirmed that DCE-MRI allows evaluation of microcirculatory changes in various disease conditions ${ }^{1} 2^{3}$. DCE-MRI generates multiple microcirculatory parameters, however, most commonly used is the "endothelial transfer coefficient' ( $K^{P S}$ or $k^{\text {trans }}$ ), and the biologic phenomenon that is most commonly evaluated is neo-vascularization in the form of angiogenesis (i.e., capillary sprouting). particularly in oncology.

At the start of this study it was not known whether low molecular (clinically safe) MR contrast agents could be efficiently used in DCE-MRI of neo-vascularization, and if so, whether it could be used in a clinical setting. Therefore, the work presented in this thesis aims to further explore DCE-MRI of neo-vasculature as potential adjunct in radiology.

DCE-MRI in oncology

The value of DCE-MRI in oncology is that it helps to objectify neo-vascular changes in tumors, for example in response to anti-vascular anti-tumor treatment ${ }^{4}$, possibly even before morphologic changes, such as reduction in tumor size, become apparent. DCEMRI might prove to be a valuable tool for tumor detection, delineating and staging, identifying the most active regions within a tumor, and for optimizing individual anti-tumor treatment ${ }^{5}$.

If DCE-MR $\|$ is to be used in humans, then it must be performed with a MR contrast agent that is safe for human use. Currently, only low molecular weight gadolinium-based contrast agents have wide clinical application, and only few high molecular weight contrast agents have a chance of becoming clinically available, for example reversible albumin binding contrast agents ${ }^{6 i}$ and iron-oxide particles (USPIO) ${ }^{7}$.USPIO particles are not only suitable for DCE-MRI, but may also be used for T1-weighted MR angiography 8 , and more importantly, for organ (tissue) specific evaluation of atherosclerosis " or for the detection of metastatic cancer in the liver or lymph nodes by $\mathrm{T} 2^{(*)}$-weighted imaging ${ }^{10,11}$. Clinically approved low molecular weight $(<1 \mathrm{kDa})$ contrast agents, on the other hand, are tissue non-specific, and were therefore thought ${ }^{12}$ to be less suited for dynamic contrast-enhanced MR imaging than high molecular weight 
contrast agents are (approximately $>24 \mathrm{kDa}$ ). The reason for this is that free diffusing low molecular weight contrast agent particles will leak even in healthy microvessels. The width of capillary pores in healthy microvessels is approximately $6-7 \mathrm{~nm}^{13}$. Although, molecular weight and particle size should not be used interchangeably. the relative permeability with respect to water (relative permeability $=1.0$ ) of low molecular weight molecules ( $<1 \mathrm{kDa}$, and $<1 \mathrm{~nm}$ in diameter) is generally greater than 0.5 . Comparatively, the relative permeability for myoglobin $(18 \mathrm{kDa})$ is 0.03 and for albumin $(70 \mathrm{kDa})$ is 0.0001 , because the approximate diameter of albumin is $6 \mathrm{~nm}^{13}$. In diseased vessels and with neo-vascular capillary sprouting (e.g. in tumors), blood vessels become highly permeable for large blood plasma molecules owing to the occurrence, for example, of capillary gaps with a with of approximately $80 \mathrm{~nm}$ and greater ${ }^{14}$.

The work presented in Chapter 2, was the first study in which the MR imaging-derived tumor microcirculation characteristics of a low molecular weight contrast agent were directly compared with those of large USPIO particles (approximate diameter, $20 \mathrm{~nm}$ ), with a generally accepted histologic validation technique. The first result of this comparison was that the effective use of USPIO for evaluating tumor neo-vascularization by DCE-MRI was re-established. Possible future advantages with the use of USPIO might be the combining of DCE-MRI and the organ (tissue) specific use of USPIO for detecting pathologic lymph nodes or liver metastases as mentioned above.

The second finding of the direct comparison was that DCE-MRI with a low molecular weight clinically available MR contrast was as good as DCE-MRI with USP\|O, in a rodent tumor model. This finding prompted us to evaluate whether DCE-MRI with the use of low molecular weight contrast agents was indeed successful for monitoring the effects of antitumor treatment (I) in rodent cancer models and (II) in patients.

The work presented in Chapter 4, showed that the effects of antitumor treatments could indeed be evaluated by DCE-MRI with the use of a low molecular weight clinically available contrast agent. The antitumor treatments that were evaluated represented a new form of antitumor strategy that specifically targeted tumor neo-vascularization. These potential of these so-called anti-angiogenesis treatments is that they might halt tumor development and metastasis formation ${ }^{4}$, and will likely be used in antitumor combination therapy, in addition to radiation therapy for example.

The work presented in Chapter 5, not only showed the successful use of DCE-MRI with a low molecular weight contrast agent for non-invasive evaluation of tumor neovascularization in rectal cancer patients. It also established the significant effects of radiation therapy allone on the tumor (neo-) microvasculature. For future studies, these results may serve as a reference for studies in which radiation therapy is combined with new anti-angiogenesis treatments. Our finding, however, that DCE-MRI derived parameters were already significantly affected by radiation therapy alone, indicates, in our opinion, that future studies ought to critically evaluate the added and/or synergistic effect new anti-angiogenesis agents when combined with radiation therapy ${ }^{45}$.

An interesting observation in Chapter 5, however, was that radiation therapy related reductions in MR imaging-derived microcirculation parameters corresponded with reductions in microvessel density but not with microvessel proliferation. This supports that DCE-MRI with a low molecular weight clinically available contrast agent does not so much reflect (angiogenesis related) microvessel permeability, but does reflect changes in microvessel (tumor) blood flow. This finding portrays one of the clues that the physiologic meaning of DCE-MRI derived parameters depends, in part, on the molecular weight of the MR contrast agent used. The implications of such results may be that one 
might choose a particular molecular weight MR contrast agent for DCE-MRI depending on the specific microcirculatory characteristics one seeks to measure.

\section{DCE-MRI in arterial occlusive disease}

To evaluate whether DCE-MRI could be used for detecting neo-vascularization related changes in muscle blood flow in a model for peripheral arterial occlusive disease, a low molecular weight contrast agent was used. This choice of contrast agent was based on the experience gained from DCE-MRI in oncology that low molecular weight agents measured microcirculatory blood flow, whereas high molecular weight agents measured microvessel permeability (-surface area). Neo-vascullarization in arterial occlusive disease is characterized by a combination of collateral vessel growth (i.e., arteriogenesils) and capillary sprouting (i.e., angiogenesis), of which the first (i.e. growth of collateral arteries) is most important for restoring muscle blood flow ${ }^{16}$. The work presented in Chapter 6 presents the first use of DCE-MRI in arterial occlusive disease for evaluating the recovery of muscle blood flow within three weeks after femoral artery ligation.

The observed changes in muscle blood flow, in healthy rabbits (Chapter 6), were strongly associated with the spontaneous growth of collateral arteries as measured using a tailored MR angiography protocol. The validity of these MR angiography measurements was evaluated with the work presented in Chapter 7 , which showed a strong association with $\mathrm{X}$-ray angiography measurements. Although less collateral arteries were typically scored with MR angiography than with $X$-ray. The lower number of collateral arteries scored with MR than with X-ray related to differences in spatial resolution, which is inherently better for $X$-ray than for MR. Unterestingly, however, by utilizing a simple flow model, it was derived that those collateral arteries scored with MR angiography accounted for $>98 \%$ of the total blood flow past the ligated artery, and that the additional smaller diameter collateral arteries scored with $X$-ray accounted only for $<2 \%$. This finding was in concurrence with conclusion stated in Chapter 6 that the changes in muscle blood flow, as measured by DCE-MRI using a low molecular weight contrast agent related to neo-vascularization in terms of collateral vessel growth.

This initial experience with serial DCE-MRI and MR angiography combined in a rabbit femoral ligation model shows its potential for monitoring anterial occlusive disease by MRI and just as with DCE-MRI in oncology, DCE-MRI will likely have additional uses in arterial occlusive disease. For example, cardiovascular disease is subject to extensive research aimed at developing therapies that promote neo-vascularization ${ }^{17,18}$. Again as with DCE-MRI in oncology, there might be a future role for DCE-MRI in evaluating early effects of such therapies, and the appropriate molecular weight of MR contrast agent might be selected depending on the direct action of these therapies. If the direct action of a vascularization promoting therapy involves increased permeability, for example. which is an early event in capillary sprouting, one might opt for the use of a high molecular weight contrast agent. The same counts for possible increases in microvessel surface area or blood wolume. Therapeutic increases in microcirculatory (muscle) blood flow might be best measured non-invasively by DCE-MRI using a low molecular weight MR contrast agent.

\section{Contrast agents for DCE-MRI}

The considerations regarding the molecular weight of commercially available MR contrast agents for DCE-MRI (Chapter 2) led us to explore more systematically the dependency of DCE-MRI results on the molecular weight of the contrast agent used. For 
this, MR contrast agents were designed, based on a dendritic scaffold (Chapter 8), that differed only in molecular weight (range: $0.7 \mathrm{kDa}$ through $51 \mathrm{kDa}$ ), but not in chemical composition, in collaboration with the Eindhoven University of Technology ${ }^{*}$. The use of these dendritic contrast agents in the rodent tumor model ${ }^{\prime}$ presented in chapter 3 . showed an inverse relationship between molecular weight of the contrast agent and the DCE-MRI derived endothelial transfer coefficient $K^{\text {PS }}$. This result can easily be understood when considering that low molecular weight contrast agents (almost) freely diffuse from blood vessels into the interstitial space, and that this rate of transfer is therefore highly dependent on the amount of blood supply. Contrarily high molecular weight contrast agents are less dependent on the amount of blood supply, but more so on the relative permeability and surface area of the microvasculature. It was concluded (1) that both low and high molecular weight contrast agents are useful for evaluating tumor angiogenesis, but (ii) that low molecular weight contrast agents are specifically suitable for evaluating microcirculatory blood flow, and (iii) that high molecular weight contrast agents are specifically suitable for evalluating microvessel permeability and surface area ${ }^{20}$.

Key to understanding the DCE-MRI derived parameters is to understand their dependency on the type of contrast agent used, not just the molecular weight of the contrast agent; but also their paramagnetic and pharmaco-kinetic properties. The most widely used paramagnetic ion in MR contrast agents is Gd(III), owing to its relatively stirong signal enhancing effect in T1-weighted MR imaging. One drawback of free Gd(III) lons is that they are toxic. For this reason MR contrast agents require that $\mathrm{Gd}(\| 11)$ is incorporated in a highly stable chelating molecular construct, that has no cellular interactions and timely renal clearance ${ }^{21}$. A second requirement of the chelating agent and the dendritic carrier molecule is that $\mathrm{Gd}(I I I)$ must be able to interact with surrounding water protons, such that water protons can approach the gadolinium atom for relaxation. Our preliminary results presented in Chapter 8 suggest that dendritic contrast agents meet these requirements. The dendritic contrast agents have favorable properties for future use in DCE-MRI, in terms of renal clearance, non-significant (blood) protein interactions, and ionic relaxivities $r_{1}$ and $r_{2}$. Other potential future applications of dendritic contrast aglents are discussed below.

\section{Limitations}

A primary limitation of DCE-MRI is that the required software necessary to perform the phamacokinetic analysis is currently not commercially available, for example from the MR vendors. The software used for the work presented in this thesis was selfprogrammed (dr. W. H. Backes). One reason for this is that the MR imaging protocol for DCE-MRI is not performed uniformly and still being optimized, worldwidle, and for different tissues and diseases. This poses another limitation, namely that the MR imaging volume of tissue coverage affects the imaging protocol for DCE-MRI, illustrated by de different $M R$ imaging protocols used for the mice and patient studies. Although, this thesis was not aimed at investigating the dependency of the DCE-MRI derived parameters of the MR protocol used, it is generally assumed that these may be affected by the time-rate of the dynamic imaging series and voxel size. Hence, the possible relationship between the micro-environmental meanings of the DCE-MRI derived parameters and MR imaging parameters will likely be the subject in future studies.

Differences between animal and patient MR protocols and between disease models and patients pose another limitation, because the results obtained in animals may not be directly extrapolated to patients. This limitation, in addition to the relatively small studies 
presented in this thesis will require further studies in larger groups of patients before a clinical use of DCE-MRI can be truly established.

\section{Future prospects}

An important consideration in MR imaging is its clinical value. Presently, the value of conventional (e.g. T1- and T2- weighted) MR imaging is well established for macroscopic evaluation of tissue pathology, for example the extent of disease in a patient with rectal carcinoma ${ }^{22}$. There is a general sentiment, however, that macroscopic changes in disease are preceded by microscopic changes, such as strong reduction in tumor perfusion that was measured just 2 weeks following radiation therapy in patients with rectal cancer, by combining functional information obtained from DCEMRI with anatomical information from conventional imaging. To move even beyond these microcirculatory changes, a newly emerging field in MR imaging involves the visualization of the cells, proteins and even molecules that are the foundation of most, if not all (disease) conditions: molecular imaging.

Molecular imaging, in general terms, refers to the visualization of tissue specific uptake of a target tracer molecule. One of the early examples of molecular MR imaging involve the uptake of USPIO contrast agent particles by cells, such as macrophages within an atherosclerotic plaque, to detect vascular disease ". More complex examples of molecullar imaging involve the combination of a MR contrast agent with a target specific probe. An example of this is the combination of a modified $\mathrm{Gd}(I I)$ chelate with a simall peptide (CNGR) (Figure 1) for targeting blood vessel endothelial cells involved in angiogenesis ${ }^{23}$.

An advantage of using Gd(III) based contrast agents over iron based USPIO particles is that $\mathrm{Gd}(\mathrm{III})$ produces a signal increasing effect, whereas USPIO produces a signal reduction that is commonly more difficult to detect. In earlier pilot studies, the
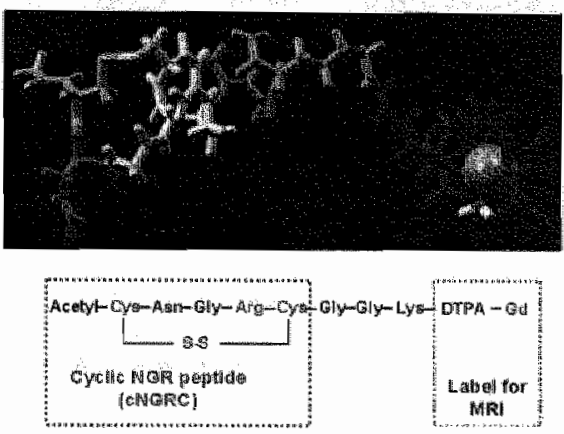

Figure 1. Sichematic presentation of cNGR-labeled MR probe. cNGR target specific contrast agent was found to accumulate in areas of high angiogenic activity, both in rodent tumors and infarcted regions in mouse hearts (Figure 2). These promising results introduced two pitfalls when considering the results in light of the work presented in this thesis.

The first pitfall using the cNGR-targeting agent was that the accumulation of the cNGR target specific contrast agent in the tumor and infarcted region in the heart was only marginally higher than that for a negative contral, non-specific MR contrast agent. One reason for these marginal results may have been the crossover design of the pilot study, in which each mouse was imaged twice with a 24-hour interval to allow for contrast agent clearance: half of the mice received the negative control contrast agent and the other mice received the cNGR targeting agent on the first day, and then the other agent on the second day. Another reason may have been that the molecular weight of the CNGR contrast agent was in the same range as the clinically approved low molecular weight contrast agent. Given the results presented in Chapter 3, the non-specific rate of transfer $\left(K^{P S}\right)$ of contrast agent from the blood into the tissue will be high for both of these agents. Differences in pharmacokinetic parameters had not been explored in this 
pillot molecular imaging study. Hence, in future molecular imaging studies, the molecular MR Imaging protocol might be combined with DCE-MRI to evaluate possible differences in $k^{p i s}$ and $/$ or reflux $(k)$ between the target specific agent and negative control, to distinguishing between tissue specific and tissue non-specific uptake.

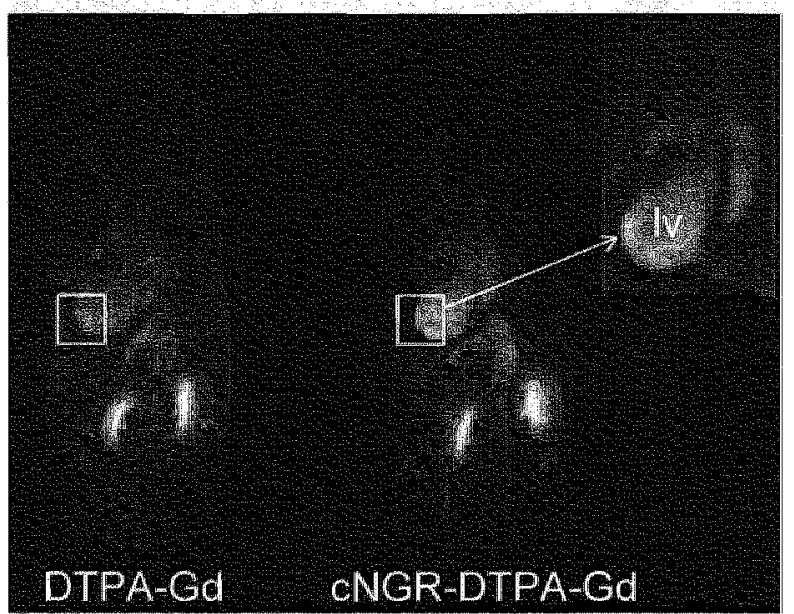

Figure 2. Contrast-enhanced MR imaging in a mouse with a 2-week-old infarction in the left ventricle (IV), imaged with a nonspecific contrast agent (DTPAGd), and imaged 24 hours later (to allow for the DTPA-Gd contrast aglent to cleared from the bady) with the target specific probe (cNGR-DTPA-Gd) shows equivalent non-specific tissue uptake.

In addition, the site of the infarction in the left ventricle shows a marginally higher signal enhancement (arrow). imaged with the target specific probe.

In order to use DCE-MRI for distinguishing between tissue specific and tissue nonspecific uptake, the pharmacokinetic model presented here in this thesis may not suffice. Non-specific leakage of a contrast agent from the blood compartment to the compartment of the extravascular extracellular space, as quantified by the twocompartment model presented in this thesis, assumed that the contrast agents used had no cellular interactions. With molecular imaging, however, the contrast agents are designed for cellular interactions, for which reason the cellular (interaction) compartment would have to be incorporated into the pharmacokinetic model, as a third compartment (Figure 3).

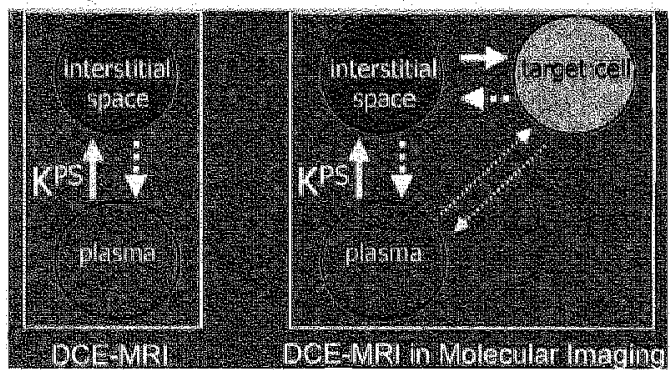

Figure 3. Schematic presentation of the general 2-compartment pharmacokinetic model used for DCE-MRI (left) illustrates that $K^{P S}$ represents the transfer coefficient (leakage) from the blood plasma to the interstitial (extravascular extracellular) space.

DCE-MRI might be of future use in Molecular Imaging by defining the exchange rate with the targeting cell to calculate the concentration of contrast agent that are successfully bound to the target cells by defining a third compartment.

The second pitfall using the cNGR-targeting agent was that after the initial phase of vast contrast enhancement of combined specific and non-specific tissue uptake the remaining signal enhancement was only marginally increased with the CNGR contrast 
agent. This could mean either that, contrary to other pllot studies using CNGR labeled thuorescent probes, there was no substantial tissue specific uptake. Or, as is illustrated in Chapter 8 , the signal enhancing effect of a single $G d(11)$ construct $\left(G d(11),-c N G R_{1}\right)$ has too high a detectable limit (almost $1000 \times$ higher than for fluorescent probes) to be detectable at low concentrations.

The large generation 5 dendrimer (G5) with 64 terminals, as presented in Chapter 8 , could be loaded with a large number of gadolinium constructs plus a number of target specific moieties, such as cNGR. This construct might just produce enough 'bulk' magnetization for molecular imaging. Chances are that 60 or so $\mathrm{Gd}(1 \mathrm{ll})$ ions per carrier might still be too few, and that larger constructs are necessary, which in turn will likely increase their toxicity.

A method for validating tissue specific uptake of the Gd(II)-CNGR construct, is to combine it with a fluorescent probe for optic fluorescent microscopy ${ }^{24}$. An ellegant model for this is the chick chorioallantoic membrane (CAM). The feasibility of performing DCEMRI in the CAM was established prewiously ${ }^{25}$. Consecutive pilot work, in collaboration with different departments at the Eindhoven University of Technology (TUE) and Maastricht University (UM), showed the feasibility of localizing gadolinium probe within the CAM (Figure 4).

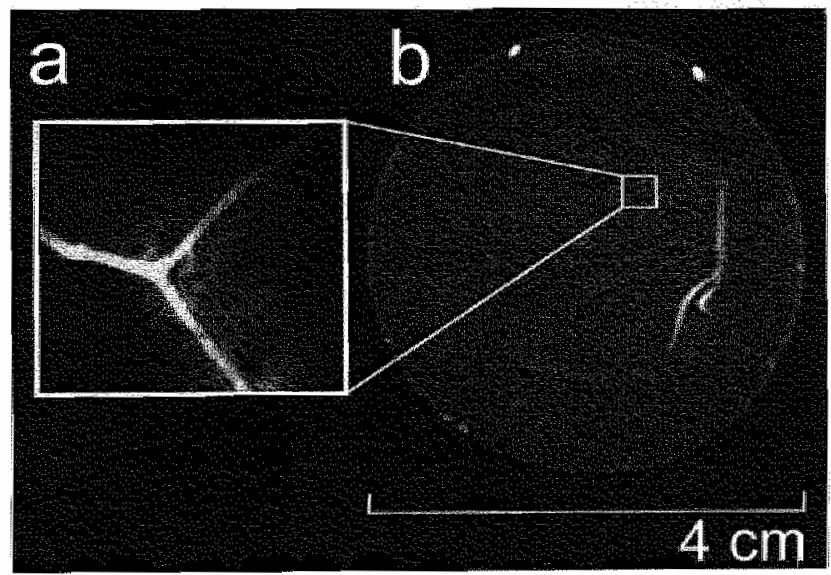

Figure 4. The chorioallantoic membrane (CAM) of the chick embryo might be ideal for direct validation of Molecular MR imaging.

Combining a targeting agent with both a MR probe and a fluorescent** probe can be used in one CAM experiment to validate by two-photon microscopy, for example (a), whether a specific region in the MR imaging volume (b) contains successful cellular targeting or non-specific contrast agent accumulation.

* Color image on back-cover.

\section{Conclusion}

The work presented in this thesis reproduces the strength of DCE-MRI as a valuable adjunct to diagnostic radiology, because it provides additional functional information in microvessel properties of various disease processes based on dynamic measurements of microvessel permeability and flow.

DCE-MRI can be performed with a specific molecular weight contrast agent depending on the micro-environmental parameter one seeks to measure. When the molecular weight is low, DCE-MRI measures microvessel blood flow; when it is high, DCE-MRI measures microvessel permeability (-surface area). This thesis showed that DCE-MRI can be performed using a clinically safe MR contrast agent. 
Given these findings, DCE-MRI is promising as a valuable tool for daily clinical practice by early detection of disease activity and monitoring treatment effects in oncology and arterial occlusive disease.

\section{References}

1. Jackson $\mathrm{A}_{\text {i }}$ Buckley DL, Parker GJilM. Dynamic contrast-enhenced magnetic resonance imaging in oncology. 1 ed. Heidelberg, Germany. Springer-Verlag; 2005.

2. van Dijke CF, Kirk BA, Peterfy CG, Genant HK, Brasch RC, Kapila S. Arthritic temporomandibular joint: correlation of macromolecular contrast-enhanced MR imaging parameters and thistopathologic findings. Radiology, 1997;204:825-32.

3. Helbich TH, Roberts TP, Rollins MD, Shames DM, Turetschek K, Hopf HW, Muhler M, Hunt TK, Brasch RC. Noninvasiwe assessment of wound-healing angiogenesis with contrast-enhanced MRI. Acad Radiol. 2002:9 Suppl 1:5145-7.

4. Griffioen AW, Molema G. Angiogenesis: potentials for pharmacologic intervention in the treatment of cancer, cardiovascular diseases, and chronic inflammation. Pharmacol Rev. 2000,52:237-68.

5. Padhani AR. Dynamic contrast-enhanced MRi in clinical oncology: current status and future directions. J Magn Reson Imaging. 2002;16:407-22.

6. Parmelee $\mathrm{DJ}$, Walowitch $\mathrm{RC}$. Ouellet $\mathrm{HS}_{\mathrm{s}}$ Lauffer RB. Preclinical evaluation of the pharmacokinetics, biodistribution, and elimination of MS-325, a blood pool agent for magnetic resonance imaging. Invest Radial. 1997;32:741-7.

7. Kroft LJ, de Roos A. Blood pool contrast agents for cardiovascular MR imaging. J Magn Reson Imaging. 1999;10:395:403.

8. Leiner $T, H o K Y, H o V B$, Bongartz $G$, Mali WP, Rasch $W$, van Engelshoven JM. Multicenter phase-II trial of safety and efficacy of NC100150 foir steadly-state contrast-enhanced peripheral magnetic resonance anglography. Eur Radiol. 2003;13:1620-7.

9. Kooi ME, Cappendijk VC, Cleutjens KB, Kessels AG, Kitslaar PJ, Borgers M, Frederik PM, Daemen $M J$, van Engelshoven JM. Accumulation of ultrasmall superparamagnetic particles of iron oxide in human atherosclerotic plaques can be detected by in vivo magnetic resonance imaging. Circulation. 2003:107:2453-8.

10. Harisinghani MG, Barentsz J, Hahn PF. Deserno WM, Tabatabaei S, wan de Kaa $\mathrm{CH}$, de la Rosette $\mathrm{J}$. Weissleder R. Noninvasive detection of clinically occult lymph-node metastases in prostate cancer. N Engl J Med. 2003;348:2491-9.

11. Verhees R, de Jong M, Beets GL, von Meyenfeldt MF, van Engelshoven JM, Beets-Tan RG. How Accurate Can Wo Predict the Nodal Status in Primary Rectal Cancer Using USPIO MRI? In: Radiological Society of North America (RSNA). Chicago, IL: 2004:SSG11-01.

12. Brasch $R_{x}$ Turetschelk $K$. MRl characterization of tumors and grading angiogenesis using tracromolecular contrast media: status report. Eur J Radiol. 2000;34:148-55.

13. Guyton $\mathrm{AC}$. The microcirculation and the lymphatic system: capillary exchange, inferstitial fluid, and lymph flow. In: Wonslewicz MJ, ed. Textbook of Medical Physiology. 8 ed. Philadelphia PA: W. B. Saunders Company: 1991:170m 184 .

14. Dvorak HF, Nagy JA, Feng $D$, Brown LF, Dvorak AM. Vascular permeability factor/vascular andothelial growth factor and the significance of microvascular hyperpermeability in angiogenesis. Curr Top Microbiol Immunol. 1999;237:97-132.

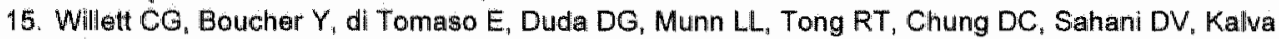
SP, Kozin SV, Mino $M_{n}$ Cohen KS, Scadden DT, Hartford AC, Fischman AJ, Clark JW, Ryan DP, Zhu AX, Blaszkowsky LS, Chen HX, Shellito PC, Lauwers GY, Jain RK. Direct evidence that the VEGF-specific antibody bevacizumab has antivascular effects in human rectal cancer. Nat Med. 2004;10:145-7.

16. Carmeliel P. Mechanisms of angiogenesis and arteriogenesis. Nat Med. 2000;6:389-95.

17. Post $\mathrm{MJ}_{\mathrm{i}}$ Simons $\mathrm{M}$. The rational phase of therapeutic angiogenesis. Minerva Cardioangiol. $2003 ; 51: 421-32$

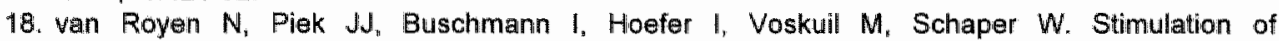
arteriogenesis; a new concept for the treatment of arterial occlusive disease. Cardiovasc Res. $2001: 49 ; 543-53$. 
19. Langereis $S$, van Genderen MP, de Lussanet $Q G$, Backøs WH, Meijer EW. Muitivalent dendritic contrast agents for Magnetic Resonance Imaging. Macromolecules. 2004. Submitted.

20. Choyke PL. Contrast agents for imaging tumor angiogenesis: is bigger better? Radiology. $2005 ; 235: 1-2$.

21. Runge VM. Safety of approwed MR contrast media for intravenous injection. I Magn Reson Imaging. 2000:12:205-13.

22. Beets-Tan RG, Beets GL. Rectal cancer: review with emphasis on MR imaging. Radiologir. $2004: 232: 335-46$.

23. Langereis $S$, Dirksen $A$, de Waal BFM, wan Genderen MHP, de Lussanet QG, Hackeng TM, Meijer EW. Solid-phase synthesis of cyclic-NGR-functionalized Gd(III)DTPA complexes for targetspecific imaging of angiogenesis. Eurpean Joumal of Organic Chemistry. 2005in press.

24. Dirksen $A$, Langereis $S$, de Waal BF, van Genderen MH, Meijer EW, de Lussanet QG, Hackeng TM. Design and synthesis of a bimodal target-specific contrast agent for angiogenesis. Org Lett. $2004 ; 6: 4857-60$.

25. de Lussanet QG, Backes WH, Beets-Tan RGH, Maas JW, Struyker Boudier HA, Evers JL, wan Engelshoven JMA. International Society for Magnetic Resonance in Medicine (ISMRM). In: International Society for Magnetic Resonance in Medicine (ISMRM). Glasgow; 2001:284. 


\section{Summary}

Dynamic contrast-enhanced magnetic resonance imaging (DCE-MRl) allows noninvasive evaluation of neo-vascularization. As DCE-MRI is increasingly used to evaluate tumor progression, we sought to better understand it and its application in oncology, and to investigate a new application for DCE-MRI in cardiovascular disease.

\section{Chapter 2}

Endothelial transfer coefficient (i,e., $K^{P S}$ values), as measured with DCE-MRI, shows equal correlation with histologic findings (i.e., MVD) when either gadopentetate dimeglumine or ultra-small iron-oxide particles (USPIO) are used.

To our knowledge, this is the first study that reveals a significant correlation between $K^{p s}$ and MVD with gadopentetate dimeglumine with use of a clinical MR imaging unit and a human colon cancer cell line inoculated in animals.

Our findings suggest that the kinetic physiologic properties of gadopentetate dimeglumine are as good as those of USP|O for calculating the endothelia transfer coefficient $K^{\text {pss }}$. Extended characterization of the tumor microenvironment, such as determination of the local blood volume fraction, might benefit more from new largemolecular contrast agents or optimized use of existing agents.

\section{Chapter 3}

DCE-MRI derived microcirculatory characteristics depend on the choice of contrast agent.

Dynamic contrast-enhanced MR imaging-derived kinetic parameters (i.e., $K^{P S}$ and $f^{P V}$ ) increase in magnitude and spatial heterogeneity as the molecular weight of dendritic contrast aglent decreases. These findings may aid in choosing the best contrast agent for dynamic contrast-enhanced MR imaging for example for evaluating effects of antiangiogenesis and other antitumor treatments. Depending on the biologic effects of the treatment strategy, high-molecular-weight contrast agents might be preferred over low-molecular-weight contrast agents in the evaluation of reductions in microvessel permeability and vascular volume. Low-molecular-weight contrast agents might be more applicable in the evaluation of therapeutic reductions in magnitude and hetterogeneity of microcirculatory blood flow.

\section{Chapter 4}

Effects of the angiostatic compounds anginex and TNP-470 on tumor angiogenesis can be assessed by DCE-MRI on a clinical 1.5 Tesla MR system and with the clinically available contrast agent gadopentetate dimeglumine.

Ouir preliminary results suggest that DCE-MRI with a clinical 1.5 Tesla MR system and a widely available, low molecular weight contrast agent may be used to evaluate the effects of angiostatic compounds on tumor angiogenesis in an animal solid tumor model.

\section{Chapter 5}

Significant radiation therapy related microcirculation changles in locally advanced rectal cancer were demonstrated by DCE-MRI and histology.

While, conventional gadolimium-enhanced MR sequences have limited additional value for morphologic diagnostic assessment of rectal cancer, new gadolinium-enhanced MR protocols, such as DCE-MRI, will complement conventional imaging of tissue morphology by non-invasive evaluation of (tumor) tissue physiology. DCE-MRI was used 
here to show that radiation therapy alone significantly inhibits angiogenesis in rectal cancer. Developments in anti-angiogenesis therapy are improving the prospects for anticancer treatment, including disseminated colorectall disease when combined with

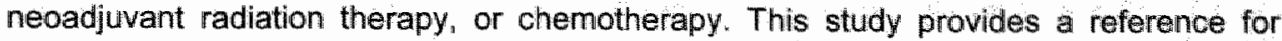
future evaluations of additive effects of anti-angiogenesis treatments that are supplemented to neoadjuvant radiation therapy in rectal cancer.

\section{Chapter 6}

Serial MR imaging demonstrated recovery of muscle perfusion and collateral vessel growth in a rabbit femoral artery ligation model.

MR imaging visualized newly formed collateral vessels by MRA complemented with quantitative perfusion information for the lower hindlimb muscles obtained by DCE-MRI. DCE-MRI showed reduction and recovery of blood inflow to the distall end of the hindlimb, after femoral artery ligation, in terms of the arterial inflow delay times, and showed reduction and recovery of muscle blood flow in terms of the endothelial transfer coefficient $\left(K^{P S}\right)$.

Recently, it was reported ${ }^{15}$ that MRI documented collateral vessel growth by MRA and in changes tissue perfusion by MR spectroscopy in patients with critical limb ischemia at three and nine months after vascular endothelial growth factor gene therapy. Considering the results of the short-term follow-up in this study, it might be hypothesized that MRI is useful for evaluating even earlier effects of treatments that stimulate neovascularization, by utilizing DCE-MRI and high spatial resolution MRA. Further work, including disease (e.g. diabetic) models may show whether DCE-MRI and MRA combined is indeed successful for evaluating peripheral arterial disease and the effects of treatments that stimulate vessel development and growth.

\section{Chapter 7}

Contrast-enhanced MR-angiography (MRA) allows for serial and non-invasive evaluation of early collateral vessel growth with sub-millimeter vessel diameters.

To our knowledge, this is the first visualization of collateral vessel growth by serial MRA in a rabbit femoral artery ligation model in correlation with XRA, and with use of a clinical 1.5 Tesla MRI system and a clinically approved MR contrast agent. The sub-millimeter sized collateral vessels that were visualized by MRA represented those arteries that were responsible ( $>98 \%$ ) for the blood flow recovery.

Further studies, for instance in diabetic models and in therapeutically enhanced neovascularization, may show the value of MRA in detecting perturbations in collateral vessel growth in ischemic disease.

\section{Chapter 8}

Dendritic contrast agents are well suited for serial contrast-enhanced MRI of sub-millimeter-sized blood vessels and evaluating tumor microcirculatory characteristics by dynamic contrast-enhanced MRI in mice.

Gd(III)DTPA-terminated poly(propylene imine) dendrimers G1, G3, and G5 show an increase of both the $T_{1}$ and $T_{2}$ relaxivities with increasing molecular weight. The results in blood plasma as described here are in good agreement with earlier measurements in citrate buffer; showing that these dendritic contrast agents have negligible interactions with blood plasma proteins. All contrast agents presented show renal clearance on dynamic contrast-enhanced MR images. Higher generations of the dendritic contrast agents display higher signal enhancement and lower concentration detection limits. 
Further research is necessary to demonstrate the efficacy of Gd(III)DTPA-functionalized poly(propylene imine) dendrimers as contrast agents for molecular MR imaging. 


\section{Samenvatting}

Gangbare MRI toepassingen zijn geschikt om macroscopische veranderingen van (ziek) weefsel te visualiseren, zoals de grootte van een tumor of vernauwingen in een bloedvat. Maar om microscopische processen te evalueren zijn meer specifieke MRI technieken nodig. Voor het aantonen van neo-vascularisatie heet deze techniek dynamisch contrastversterkte MRI, ofwel DCE-MRI.

\section{Neo-vascularisatie}

Neo-vascularisatie betreft de ontwikkeling en groei van bloedvaten op het niveau van de microcirculatie. Specifieke onderdelen van neo-vascularisatie zijn de nieuwvorming van haarvaatjes (capillairen) en de groel van collateraal arteriën uit preexistente vaatverbindingen. Neo-vascularisatie is essentieel in de vroege ontwikkelingsfase van een kwaadaardige tumor, maar ook in de vroege herstelfase na een vaatafsluiting bij hart- en vaatziekten. Er zijn tegenwoordig dan ook therapieün die neo-vascularisatie kunnen remmen (te gebruiken bij kwaadaardige tumoren), ofwel stimuleren (te gebruiken bij hart- en vaatziekten).

\section{DCE-MRI}

DCE-MRI is gebaseerd op het gedurende meerdere minuten continu herhalen van een bepaalde MRI meting. Tijdens deze dynamische meting wordt een contrastmiddel intraveneus toegediend. Vervolgens zal het contrastmiddel door het lichaam circuleren en signaalveranderingen teweegbrengen in het bloed en in verschillende weefsels. Door deze signaalveranderingen te vertalen naar concentratieveranderingen kan met behulp van een farmacokinetisch model onder andere de snelheid worden berekend waarmee het contrastmidddel vanuit de bloedbaan naar het omgevende weefsel lekt (het interstitium, ofwel extravasculaire ruimte). Deze zogenaamde 'endothelial transfer coefficient' $K^{P S}$ (eenheid: mmol per minuut per $100 \mathrm{~cm}^{3}$ weefsel) is de grootheid die centraal staat in dit proefschrift.

\section{Geschikte contrastmiddelen voor DCE-MRI}

In het verleden werd DCE-MRI uitsluitend in proefdieren uitgevoerd en daarbij werd gebruik gemaakt van experimentele contrastmiddelen die niet toegestaan waren voor gebruik in mensen. Deze contrastmiddelen bestaan uit relatief grote partikels die traag door het lichaam worden uitgescheiden en vrijwel alleen in weefsels die neovascularisatie vertonen naar het interstitium lekken. Deze DCE-MRI techniek kon dus niet op mensen worden toegepast. De in de dagelijkse klinische praktijk gebruikte contrastmiddelen bestaan echter uit kleine partikels die snel door het lichaam worden uitgescheiden en ook snell naar zowel het interstitium van weefsels die neovascularisatie bevatten als van gezond weefsel lekken waardoor onvoldoende contrast tussen gezond en ziek zou ontstaan. Deze klinische contrastmiddelen zouden daardoor mogelijk ongeschikt zijn voor DCE-MRI. Het doel van de studie in Hoofdstuk 2 was om te toetsen of zo'n klinisch beschikbaar en dus klein moleculair contrastmiddel niet ook geschikt zou kunnen zijn voor DCE-MRI. Hiervoor werden de DCE-MRI resultaten bij gebruik van een klein moleculair MR contrastmiddel Gd-DTPA ( gebaseerd op het paramagnetische gadolinium ion) vergeleken met de DCE-MR\| resultaten bij gebruik van een relatief groot contrastmiddel "USPIOs" (partikels met een superparamagnetische ijzerkern). Er werd gekozen om gebruik te maken van het meest gangbare model voor tumor neo-vascularisatie, namelijk muizen met een onderhuidse tumor. 
Beide contrastmiddelen toonden een even sterke en significante correlatie tussen de DCE-MRI parameter $K^{P S}$ en de microvaatdichtheid (MVD) als maal woor neovascularisatie: Op basis van deze resultaten werd geconcludeerd dat het klinische beschikbare klein moleculaire MR contrastmiddel Gd-DTPA even geschikt is woor gebruik bij DCE-MRI als het experimentele groot moleculaire MR contrastmiddel USPIO.

\section{$K^{p s}$ is afhankelijk van het molecuulgewicht van het contrastmiddel}

Ondanks dat verschillende MR contrastmiddelen evengeschikt bleken bestond toch het idee dat de $K^{P S}$ afhankelijk is van het soort contrastmiddel en dan met name van het molecuulgewicht. Immers naast de verschillen in snelheid van uitscheiding via de urine, zullen kleine contrastmiddelen (laag molecuul gewicht) mogelijk sneller lekken vanuit de biloedbaan naar het interstitium dan groot moleculaire contrastmiddelen.

Om deze vraagstelling te benaderen werd (Hoofdstuk 3) nogmaals hetzelfde experiment gedaan als beschreven in hoofdstuk 2 maar nu met dendritische contrastmiddelen wan verschillende grootte die zijn ontwikkeld in samenwerking met de vakgroep Macromoleculaire Chemie van de Technische Universiteilt, Eindhoven. Deze dendritische contrastmiddelen hadden een oplopend molecuulgewicht $(0,7,3,12$ en 51 $\mathrm{kDa}$ ) maar waren verder geheel identiek qua chemische samenstelling.

De resultaten toonden een omgekeerde relatie tussen $K^{P S}$ en het molecuulgewicht van de gebruikte contrastmiddelen. Met andere woorden: hoe lager het molecuulgewicht, hoe hoger de $K^{\mathrm{pS}}$. Op basis van de gevonden resultaten werd geconcludeerd dat de $K^{\mathrm{pS}}$ bij gebruik van grote contrastmiddelpartikels voornamelijk de daadwerkelijke permeabiliteit van het vaatwandoppervlak weergeeft, en dat de $K^{p S}$ bij gebruik van kleine partikels voornamelijk de mate van doorbloeding reflecteert (in combinatie met de permeabiliteit').

DCE-MRI meet effecten van antitumor therapieën op de neo-vascularisatie in experimentele setting.

De bevindingen uit Hoofdstuk 3 kunnen van bellang zijn voor de keuze van het contrastmiddel bij DCE-MRI onderzoek van neovascularisatie ter beoordeling van bepaalde therapeutische effecten. Eerdere DCE-MRI studies konden bijvoorbeeld korte termijn effecten van een therapie die de neo-vascularisatie remt (anti-VEGF) enkel aantonen met relatief grate on niet met kleine contrastmiddelen.

In Hoofdstuk 4 werd daarom getoetst of DCE-MRI met een klein moleculair contrastmiddel inderdaad geschikt is om de effecten van antivasculaire antitumor theirapieen (anginex en TNP-470) te kwantificeren. De resultaten toonden lagere $K^{P S}$ waarden in tumoren van muizen die behandeld waren met anginex of met TNP-470, in vergelijking met die van onbehandlelde muizen.

\section{DCE-MRI meet effecten van radiotherapie in patienten met endeldarmkanker}

Antivasculaire antitumor therapieën hebben inmiddels hun entree in de patiëntenzorg gemaakt omdat gebleken is dat dergelijke therapieèn effectief ziln bij de behandeling van patiënten met kwaadaardige vormen van darmkanker. DCE-MRI zou dan een geschikte techniek zijn om de effecten van deze therapieën te evalueren. De mogelijke meerwaarde van DCE-MRI is dat vroege effecten van de therapie gemeten kunnen worden alvorens macroscopische veranderingen zoals afname van de tumorgrootte meetbaar zijn. Maar er zijn ook bestaande kanker therapieën, zoals radiotherapie die een effect hebben op de neo-vascularisatie. Er zijn zelfs berichten dat nieuwe antivasculaire therapieën een symergistische werking zouden kunnen hebben op 
radiotherapie en dat deze dus als radiosensitizer zou kunnen clienen. Het onderzoek in Hoofdstuk 5 bepaalt bij patiènten met endeldarmkanker het afzonderlike effect van neo-adjuvante radiotherapie op de tumor doorbloeding.

De resultaten toonden dat radiotherapie inderdaad een significante $K^{\text {ps }}$ reductie teweeg brengt in bestraalde tumoren en dus dat radiotheraple de tumordoorbloeding doet afnemen. Deze bevindingen geven een mogelijke referentie voor toekomstige studies waar antivasculaire theraple als radiosensitizer toegevoegd zal worden aan reguliere neoadjuvante radiotherapie.

\section{DCE-MRI van spierdoorbloeding na vaatafsiluiting}

Met de nodige opgedane ervaring met DCE-MRI in tumoren werd de stap gemaakt naar DCE-MRI in perifeer vaatijiden. Hiervoor werd een konijnenmodel voor occlusief vaatlijden geadopteerd. In dit model wordt de slagader in de lies (femoraal arterie) chirurgisch afgesloten, met als gevolg dat de splerdoorbloeding in de kuit- en scheenspieren acuut afneemt en pas na meerdere weken herstelt. In Hoofdstuk 6 werd getoetst of DCE-MRI geschikt zou zijn om veranderingen in spierdoorbloeding te meten, door gebruik te maken van een klein moleculair contrastmiddel.

De resultaten toonden een sterke daling van de spierdoorbloeding $K^{\text {ps }}$ direct na afbinden vain de femoraal arterie ten opzichte van de controle poot. $\mathrm{Na} 7$ dagen was de doorbloeding ( $K^{P S}$ waarde)gedeeltellik en na 21 dagen geheel hersteld.

Er werd geconcludeerd dat DCE-MRI geschikt is om verandering in spierdoorbloeding te meten in een model woor vaatafs/uiting.

\section{$M R I$ van collateraal arterièn}

De groei van collateraal arteriën rondom de vaatafs/uiting wordt gezien als belangrijkste oorzaak van het herstel in spierdoorbloeding na afsluiting van de femoraal arterie (Hoofdstuk 6). In Hoofdstuk 7 werd getoetst of een aangepaste MR angiografie (MRA) meting geschikt is om deze collateraal arteriën af te beelden. Het voordeel van een dergelijke meting is dat het eenvoudig is te combineren met een DCE-MRI meting, in eenzelfde sessie. Ondanks dat MRA minder collateralen afbeeldde dan röntgenangiografie dit omdat de röntgentechniek meer detail kan afbeelden (ongeveer $0,15 \mathrm{~mm}$ ) dan MR angiografie (ongeveer $0,3 \mathrm{~mm}$ ), was er toch een hoge associatiecoefficiënt tussen de twee beeldvormende technieken. Uit een eenvoudige modelberekening, gebaseerd op laminaire doorbloeding bleek dat de submillimeter dikke arteriën die met MR angiografie (en ook met röntgenangiografie' werden afgebeeld voor meer dan $98 \%$ aan de bloedtoevoer bijdragen.

Deze bevindingen geven aan dat MR angiografie in staat is om collateraal arteriên af te beelden die verantwoordelijk zijn woor het herstel van de bloedtoevoer in een model voor perifeer vaatlijden.

\section{Karakterisening van dendritische contrastmiddelen}

Al werd in de bovenstaande hoofdstukken vooral de nadruk gelegd op de grootte van het contrastmiddiel, er resteren nog meer onderlinge verschilien tussen de genoemde contrastmiddelen die van belang kunnen zilin voor toepassing met MRI. Voorbeelden hiervan zijn het relatieve signaal versterkende effect van de moleculen, de circulatie tijd. onderlinge interactie en interactie met bijvoorbeeld bloedeiwitten. Deze eigenschappen werden geïnventariseerd voor de dendritische contrastmiddelen in Hoofdstuk 8. Geen van deze dendritische contrastmiddelen toonde interacties met bloedplasma eiwitten. Elk van de dendritische contrastmiddelen toonde uitscheiding via de urine (nieren en 
blaas), wat een gunstig gegeven is in verband met toxiciteit. Een interessante bevinding was dat de hogere generaties dendritische contrastmiddelen (hoog molecullgewicht) een betere signaalversterking geven bij lage concentraties dan lage generaties (laag molecuulgewicht), en bovendien een langere circulatietijd hebben. Deze eigenschappen zouden de hoge generaties dendritische contrastmiddelen aantrekkelijk maken voor MRA van nog kleinere collateraal arteriën dan aangetoond in de studie beschreven in Hoofdstuk 7. Een hoge signaalversterking bij lage concentraties is tevens essentieel voor nieuwe ontwikkelingen in MRI op het gebied van moleculaire beeldvorming (molecular imaging), waarmee bepaalde biochemische substanties karakteristiek voor de onderliggende pathologie worden gedetecteerd, bijvoorbeeld door interactie tussen en contrastmiddel en ziekte specifieke receptormoleculen op een celoppervlak.

\section{Conclusie}

Dit proefschrift laat zien dat dynamisch contrastversterkte MRI (DCE MRI) een belangrijke toevoeging kan zijn aan de conventionele morfologische MR beeldvorming door functionele informatie te verschaffen over neo-vascularisatie in patienten met kwaadaardige tumoren en occlusief vaatlijden. 


\section{Acknowledgements}

My gratitude goes out to my promoter professor Jos van Engelshoven and co-promoters Walter Backes and Regina Beets-Tan. Thank you for selecting me for this research project and for your teachings and encouragement during this fine period that led to the completion of this thesis. Ihope we will continue to work together in future projects.

I thank the members of my review committee for their efforts in reviewing this thesis.

Dear friends and colleagues ** I thank you for sharing your skills and expertise, and for your friendship, I'm sure this thesis would not have not have come to its existence without the help of your combined efforts.

I thank my former and current teachers and mentors for their guidance throughout my studies.

I thank my closest family Corey Hermance, Michelle, and Marianne for their unconditional support.

**

Sander Langereis, Bert Meijer, Marcel van Genderen and Anouk Dirksen, Laboratory of Macromolecular Chemistry. Eindhoven University of Technology, thank you for all your efforts that led to the success of our combined projects. Sander it was great working with you.

Arjan Griffioen, Daisy van der Schaft, Coen Baeten, Loes van Eijk and many of the student researchers, Angiogenesis Laboratory, Maastricht University, thank you for teaching me the laboratory skills involved with angiogenesis models. The doors to your office and lab always were open.

Jolanda van Golde, Maya Huijberts and Nicolaas Schaper, Department of Internal Medicine, Maastricht University Hospital, thank you for working with me on the peripheral ischemia model. Jolanda your dedication to your work is admirable. Andries Gilde and Jos thank you for pitching in from time to time.

Alexandira Buehler, Henny Schulten and Mark Post, Department of Physiology, Maastricht University, thank you for your help in learning the peripheral ischemia model and involving me in your cardiac ischemia experiments.

May, Petra, Joyce, Richard, Nadien and other technicians and staff members of the animal research facilities (CPV), Maastricht University, thank you for your continuing efforts in the surgery facilities and in caring for the animals.

Tilman Hackeng, Department of Biochemistry, Maastricht University, thank you for sharing your lab (mainly with Sander) for our joint project - and helping me remember San Diego. 
Jacques Maas, Department of Gynecology, Maastricht University Hospital, and Helma, technician, Department of Pharmacology, Maastricht University, thank you for your help on the CAM experiments

Fons Kessels, KEMTA, Maastricht University, thank you for sharing your expertise on statistical analyses.

Jaq, Chris, Michiel de Haan and colleagues at the Angiography Department, Maastricht University Hospital, thank you for your efforts in setting up our peripheral ischemia model.

Marc van Zandwoort at the Department of Biophysics, Maastricht University it was good working with you.

Jos Smits at the Department of Pharmacology, Maastricht University, thank you your advise over the years.

Geerard Beets and colleagues at the Department of Surgery, Maastricht University Hospital, thank you for helping with the patient inclusion.

Arjan de Bruine and laboratory technicians at the Department of Pathology, Maastricht University Hospital, thank you for your help with my histological analyses.

Angela van Baardwijk and professor Lambin, Maastro Clinic, Maastricht, thank you for your help with our patient study.

Fellow Radiology researchers at Maastricht University Hospital: I wish you all the best in your future endeavors. Dave it was great to have you as my roommate these past years. Vera, Suzanne, Robbert, Nils, Robbert-Harm, Marc, Luc, Jaap, Rianne, Max and Sanne, good luck with your thesis'. Marlies all the best with (molecular) MR imaging of neovasculature in cardiovascular disease. Daniëlle Zaar thanks for helping with the angiography comparisons.

Radiology research staff: Marc Geerlings, Eline Kooi and Gerrit Kemerink thank you for sharing your technical experitse.

MRI technicians, in particular Henk, Etienne, Liesbeth, and your colleagues; Radiologists; colleague residents, in particular Tim, Boudewijn and Vincent; administrative staff in particular Ine and Herman; and secretary staff in particular Brigitte thank you for your help with my research over the years. Roy vliegen, thank you for helping me evaluate pelvic MRII.

Klaas Nicolay, Department of Biomedical Engineering, Eindhoven University of Technology, thank you for sharing your insights in MR imaging.

Anwar Padhani, Paul Strickland Scanner Centre, Mount Vernon Hospital "Northwood, Middlesex, United Kingdom, thank you for sharing your expertise on DCE-MRI. 


\section{Curriculum vitae}

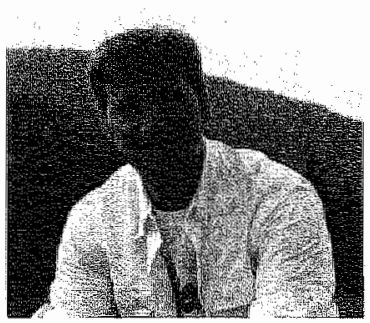

Quido Gisou de Lussanet de la Sablonière was born September 27, 1973, in Amersfoort, The Netherlands.

In the summer of 1989 he immigrated to the United States. He graduated with the class of 1990 from Mountain Empire High School, Pine Valley, California, with honors, and was recipient of the Bank of America Achievement Award in the field of applied arts and the Presidential Academic Achievement Award.

In 1993 he obtained his Associates Degree (A.S.) in Business Administration with honors, at Grossmont College, El Cajon, California, and completed the requisite premedical courses.

He enrolled Maastricht University School of medicine, Maastricht, The Netherlands, in 1994 and received his medical doctor degree in 2000. During his studies he completed elective research projects in computer phonetography, with $d r$. R. Buekers at the Ear Nose and Throat (ENT) Department, Maastricht University Hospital in 1996; and in MR imaging of rectal wall invasion of endometriosis, with dr. R.G.H. Beets-Tan in 2000 at the Department of Radiology, Maastricht University Hospital.

The author started his Ph.D. research program, entitled MR imaging of neo-vasculature, in the fall of 2000 . In 2004 he started his residency training at the Department of Radiology, Maastricht University Hospital. 


\section{List of publications}

The designer antiangiogenic peptide anginex targets tumor endothelial cells and inhibits tumor growth in animal models,

van der Schaft D.W., Dings R.P., de Lussanet Q.G., van Eilk L.I., Nap A.W., Beets-Tan R.G.H., Bouma-ter Steege J.C., Wagstaff J., Mayo K.H. \& Griffioen A.W. Faseb J. $2002 ; 16: 1991-1993$.

Gadopentetate dimeglumine versus ultrasmall superparamagnetic iron oxide for dynamic contrast-enhanced MR imaging of tumor angiogenesis in human colon carcinoma in mice,

de Lussanet Q.G., Backes W.H., Griffioen A.W., van Engelshoven J.M.A. Beets-Tan R.G.H. Radiology. 2003;229(2):429-438.

Dynamic contrast-enhanced magnetic resonance imaging at 1.5 Tesla with gadopentetate dimeglumine to assess the angiostatic effects of anginex in mice.

de Lussanet Q.G., Beets-Tan R.G.H., Backes W.H. van der Schaft D.W., van Engelshoven J.M.A., Mayo K.H., Griffioen A.W. Eur J Cancer. 2004;40(8):1262-1268.

Design and synthesis of a bimodal target-specific contrast agent for angiogenesis, Dirksen A., Langereis S., de Waal B.F.M., van Genderen M.H.P. Meljer E.W., de Lussanet Q.G., Hackeng T.M.. Organic Letters. 2004;6(26): 4857-4860.

Multivalent contrast agents based on Gd-DTPA-terminated poly (propylene imine) dendrimers for Magnetic Resonance Imaging,

Langereis S., de Lussanet Q.G., van Genderen M.H.P., Backes W.H., Meijer E.W., Macromolecules, 2004; (37):3084-3091.

Towards target-specific molecular imaging of angiogenesis with Gd(III)DTPA-based dendritic architectures,

Langereis S., de Lussanet Q.G., van Genderen M.H.P., Backes W.H., Hackeng T.M., van Engelshoven J.M.A. Meijer E.W., Polymeric Materials Science Engineering 2004; (6) $4857-4860$.

Dynamic Contrast-enhanced MR Imaging Kinetic Parameters and Molecular Weight of Dendritic Contrast Agents in Tumor Angiogenesis in Mice,

de Lussanet Q.G., Langereis S., Beets-Tan R.. G., van Genderen, M.H., Griffioen, A.W., van Engelshoven "J.M., Backes, W.H., Radiology 2005;235:65-72.

Solid-phase synthesis of cyclic-NGR-functionalized Gd(III)DTPA complexes for targetspecific imaging of anglogenesis,

Langereis S., Dirksen A., de Waal B.F.M., van Genderen M.H.P., de Lussanet Q.G., Hackeng T.M., Meijer E.M., European Journal of Organic Chemistry 2005; in press.

Dynamic contrast-enhanced magnetic resonance imaging of radiation therapy induced microcirculation changes in rectal cancer,

de Lussanet Q.G., Backes W.H., Griffioen A.W. Padhani A.R., Baeten C.I., van Baardwijk A., Lambin P.. Beets G.L., van Engelshoven J.M.A., Beets-Tan R.G.H., International Journal of Radiation Oncology Biology Physics (Red Journal) 2005; E-pub. 
Magnetic resonance angiography of collateral wessel growth in a rabbit femoral artery ligation model

de Lussanet Q.G. van Golde J.C.G, Beets-Tan R.G.H, de Haan M.W., Zaar D.V.J., Post M.J., Huijberts M.S., Schaper N.C., van Engelshoven J.M.A., Backes W.H., NMR in Biomedicine 2005, in press.

non-W Intemet publication:

Diriesen R., Everon Z., de Lussanet Q., Majidi M., Michilesen E., Narolska N., Roos, van Eys $\mathrm{G}$. Cloning of a porcine smoothelin cDNA. NCBI Nucleotide database, 2004 Mar; organism: sus scrofa, version AY547544.1, Gl:45154852. 


\section{Proceedings}

\section{Presentations:}

de Lussanet Q., Backes W., Beets-Tan R, Griffioen A, van Engelshoven J., Plenary lecture: Dynamic Contrast-Enhaced MRl of Tumor Angiogenesis; Comparing a Small and a Large Molecular Contrast Agent; 88th annual meeting, Radiological Society of North America (RSNA) 2002, p47, Chicago.

Beckers M. de Lussanet Q. Dunselman G., Beets G., Vliegen R. van Engelshoven J., Bevts-Tern R., MR: for assessment of rectal wall invasion in endometriosis, 88th annual meeting, Radiological Society of North America (RSNA), 2002, p446 Chicago.

de Lussanet Q., Beets-Tan R., Dunsetman G., Beets G., Vliegen R., Kessels A. van Engelshoven J.. $_{\text {. }}$ $M R \|$ for assessment of rectal wall inwasion in endometriosis, Annual meeting, European Conference of Radialogy (ECR), 2002, Vienna.

de Lussanet Q. Backes W., Beets-Tan R., Griffioen A., van Engelshoven J., Dynamic ContrastEnhanced MRI of Tumor Angiogenesis, Comparing a Small and a Large Molecular Contrast Agent; 7 th annual meeting, Radiological Society of The Netherlands, 2002, Noordwijkerhout.

de Lussanet Q., Backes W., van der Schaft D., Mayo K., Griffioen A., van Engelshoven J." Beets-Tan

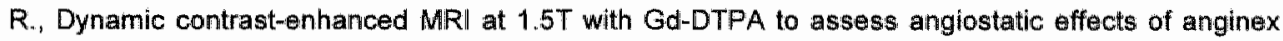
treatment in mice, 8th annual meeting. Radiological Society of The Netherlands, 2003, Noordwijkerhout.

de Lussanet Q., Beets-Tan R., Backes W. van der Sichaft D., van Engelshoven J, Mayo K., Griffioen A. Dynamic contrast-enhanced MRI at $1.5 \mathrm{~T}$ with Gd-DTPA measures angiostatic effects in tumors after treatment with anginex, 20th annual meetig, European Society of Magnetic Resonance in Medicine and Biology (ESMRMB) 2003, Rotterdam.

de Lussanet Q. Langereis S. Beets-Tan R., van Genderen M., Griffioen A., Smits J., Meijer E., van Engelshoven J., Backes W., Dynamic contrast-enhanced MRI of tumor angiogenesis with different generations of Gd-DTPA terminated dendrimers, 20th annual meetig "European Society of Magnetic Resonance in Medicine and Biology (ESMRMB) 2003, Rotterdam.

Backes W. de Lussanet Q. Beets-Tan R., Griffioen A., van Engeishoven J., Dynamic MRI of Tumor Angiogenesis: Contrast Agent Selection and Treatment Diagnosis, 30th annual meeting, European Federation of Organizations for Medical Physics (EFOMP) 2003, Eindhoven.

de Lussanet Q., Langereis S. Beets-Tan R., Grifficen A., wan Genderen M., Meijer E., van Engelshoven J., Backes W. MR imaged characteristics of tumor angiogenesis dempends on molecular size of contrast agents, 12th annual meeting, International Society for Magnetic Resonance in Medicine (ISMRM) 2004, p2370, Kyoto.

de Lussanet Q., Backes W., Baeten C., Beets G., van Engelshoven J., Beets-Tan R., Dynamic Contrast-enhanced MRI Combined with Pharmacokinetic Modeling Can Monitor Effects of Neoadjuvant Radiation Therapy in Patients with Curable Primary Rectal Cancer Compared with Histologic CD31/34 and Ki67 Analysis, 90th annual meeting, Radiological Society of North America (RSNA), 2004, p435, Chicago. 
de Lussanet, Q., Backse, W. Baeten, C., Beets, G. Bruine, A. de, Grifioen, A., Engetshovem, J. vain, Beets-Tan, R. Dynamic contrast-enhaniced MRI combined with pharmacokinetic modeling can monitor effects of neoadjuvant radiation therapy in patients with curable primary rectal cancer compared with histology, 9th annual meeting, Radiological Society of The Netherlands, 2004, Noordwijkerhout.

Langereis S., de Lussanet Q, van Genderen M., Backes W., Hackeng T., van Engelshoven J., Meijer E. Towards target-specific molecular imaging of angiogenesis with Gd-DTPA-based dendritic architectures, 228th American Chemical Society (ACS) National Meeting, 2004; Polymeric Materials, 2004(91), 56-57 Philadelphia.

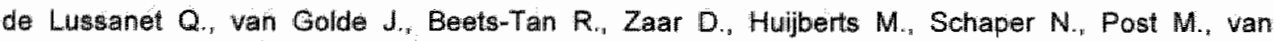
Engelshoven J., Backes W., Angiogenesis and arteriogenesis evaluation by DCE-MRI and CE-MRA in a peripheral ischemia model, 12th annual meeting. International Society for Magnetic Resonance in Medicine (ISMRM) 2005; Miami.

de Lussanet $Q_{*}$ van Golde J., Beets-Tan R., Post M., Huijberts M., Schaper N., Kessels A., van Engelshoven J., Backes W. MR monitoring of collateral growth and perfusion recovery in a rabbit femorial artery ligation model, gith annual meeting, Radiological Society of North America (RSNA), 2005, Chicago.

\section{Posters:}

de Lussanet Q., Backes. W. Beets-Tan R., Maas J., Struyker-Boudier H., Evers J., van Engelshoven J.. A Model for Mapping Angiogenesis using Dynamic Contrast-Enhanced MRI using the chick chorioallantoic membrane. 10th annual meeting. International Society for Magnetic Resonance in Medicine (ISMRM) 2001, p2253, Glasgow.

de Lussanet Q., Backes W., Beets-Tan R., Griffioen A., van Engelshoven J., DC MRI of Angiogenic Tumors in Mice with Histologic Correlation using CD31 antibody; 11th annual meeting, International Society for Magnetic Resonance in Medicine (ISMRM) 2002, p2110, Honolulu.

de Lussanet Q., Beets-Tan R., Backes W., van der Schaft D. van Engelshoven J., Mayo K.. Griffioen A., Dynamic contrastenhanced MRI at $1.5 \mathrm{~T}$ with Gd-DTPA measures angiostatic effects in fumors after treatment with anginex, 11 th annual meeting, International Society for Magnetic Resonance in Medicine (ISMRM) 2003; p823, Toronto.

de Lussanet Q., Langereis S, Beets-Tan R., van Genderen M., Griffioen A., Smits J." Meijer E., van Engelshoven J., Backes J., Dynamic contrast-enhanced MR\| of tumor angiogenesis with generations G0, G1, G3, and G5 poly(propylene imine) contrast agents, 11th annulal meeting, International Society for Magnetic Resonance in Medicine (ISMRM) 2003; p1239, Toronto.

de Lussanet Q. Backes W. Griffioen $A_{\text {. }}$ Baeten $C_{.,}$Beets $G_{. "}$ van Engelshoven J., Beets-Tan R.; Effects of neoadjuvant radiation therapy in rectal cancer assessed by dynamic contrastenhanced MRI and immunohistochemistry. 15th Endothelial Cell Research Symposium 2004, Maastricht. Angiogenesis, 2004 7(2):165-187.

Zaar D., de Lussanet Q., van Golde J., Beets-Tan R., Backes W., van Engelshoven J., Micro-MRA of Arteriogenesis: the growth of tortwous sub-millimeter collaterals in a peripheral ischemia model. Magnetic Resonance Angiography Workshop, 2004, 113, London, Ontario. 


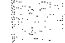

